Florida International University

FIU Digital Commons

FIU Electronic Theses and Dissertations

University Graduate School

$3-17-2020$

\title{
Novel Passive RFID Temperature Sensors Using Liquid Crystal Elastomers
}

Yousuf Shafiq

yshaf001@fiu.edu

Follow this and additional works at: https://digitalcommons.fiu.edu/etd

Part of the Electrical and Electronics Commons, Electromagnetics and Photonics Commons, Polymer and Organic Materials Commons, and the Systems and Communications Commons

\section{Recommended Citation}

Shafiq, Yousuf, "Novel Passive RFID Temperature Sensors Using Liquid Crystal Elastomers" (2020). FIU Electronic Theses and Dissertations. 4379.

https://digitalcommons.fiu.edu/etd/4379

This work is brought to you for free and open access by the University Graduate School at FIU Digital Commons. It has been accepted for inclusion in FIU Electronic Theses and Dissertations by an authorized administrator of FIU Digital Commons. For more information, please contact dcc@fiu.edu. 


\title{
FLORIDA INTERNATIONAL UNIVERSITY
}

Miami, Florida

\section{NOVEL PASSIVE RFID TEMPERATURE SENSORS USING LIQUID CRYSTAL ELASTOMERS}

\author{
A dissertation submitted in partial fulfillment of \\ the requirements for the degree of \\ DOCTOR OF PHILOSOPHY \\ in \\ ELECTRICAL AND COMPUTER ENGINEERING \\ by
}

Yousuf Shafiq 
To: $\quad$ Dean John L. Volakis

College of Engineering and Computing

This dissertation, written by Yousuf Shafiq, and entitled Novel Passive RFID Temperature Sensors Using Liquid Crystal Elastomers, having been approved in respect to style and intellectual content, is referred to you for judgment.

We have read this dissertation and recommend that it be approved.

\begin{tabular}{rr}
\hline Nezih Pala \\
\hline Elias Alwan \\
\hline Berrin Tansel \\
\hline Taylor Ware \\
\hline Stavros V. Georgakopoulos, Major Professor
\end{tabular}

Date of Defense: March 17, 2020.

The dissertation of Yousuf Shafiq is approved.

Dean John L. Volakis College of Engineering and Computing

Andres G. Gil

Vice President for Research and Economic Development and Dean of the University Graduate School

Florida International University, 2020 
(C) Copyright 2020 by Yousuf Shafiq

All rights reserved. 


\section{DEDICATION}

I dedicate this dissertation to my family, especially my Mother and Father. Without their prayers and support, the completion of this work would never have been achievable. 


\section{ACKNOWLEDGMENTS}

I would like to convey my sincerest gratitude to my major professor, Dr. Stavros V. Georgakopoulos for the opportunity to complete my doctoral degree at FIU. I thank Professor Georgakopoulos for his patience and availability to always help me when needed. I am appreciative for his guidance, advice, and experience that he has shared with me throughout this experience. It was always a pleasure to discuss and trade technical ideas and concepts. I admire his imagination and tenacity to continuously reinvent himself and derive new ideas for future research. Finally, I am fortunate to work Dr. Georgakopoulos as his lab presents an enriching research environment with excellent computational capability using the latest research software along with modern testing and fabrication equipment.

Furthermore, I am thankful to Professor Taylor Ware and his students: Kim Hyun, Cedric Ambulo, and Julia Henricks at the University of Texas at Dallas. They have been pivotal to the success of my research as they embraced my ideas and accordingly provided novel Liquid Crystal Elastomer (LCE) designs. I am grateful for their patience and punctuality in teaching me the basic concepts of LCEs and providing the LCE samples in a timely manner, respectively. Moreover, I am appreciative for their hospitality in accommodating me during my visit to their laboratory. I also appreciate Dr. Nezih Pala, Dr. Elias Alwan, and Dr. Berrin Tansel for serving on my dissertation defense committee and for their illuminating comments.

I also thank all the members of FIU Electrical and Computing Engineering Department and special thanks to the Post-Docs and students at the Transforming Antennas Center (TAC) at FIU along with Mr. Oscar Silveira for their help and friendship. Finally, 
I would also like to recognize the National Science Foundation for their support of this work. 


\section{ABSTRACT OF THE DISSERTATION \\ NOVEL PASSIVE RFID TEMPERATURE SENSORS USING LIQUID CRYSTAL ELASTOMERS}

by

Yousuf Shafiq

Florida International University, 2020

Miami, Florida

\section{Professor Stavros. V. Georgakopoulos, Major Professor}

When transporting perishable foods in the Cold Supply Chain (CSC), it is essential that they are maintained in a controlled temperature environment (typically from $-1^{\circ}$ to $10^{\circ} \mathrm{C}$ ) to minimize spoilage. Fresh-food products, such as, meats, fruits, and vegetables, experience discoloration and loss of nutrients when exposed to high-temperatures. Also, medicines, such as, insulin and vaccines, can lose potency if they are not maintained at the appropriate temperatures. Consequently, the CSC is critical to the growth of global trade and to the worldwide availability of food and health supplies; especially, when considering that the retail food market consists mostly (approximately 65\%) of fresh-food products.

The current method of temperature monitoring in the CSC is limited to discrete location-based measurements. Subsequently, this data is used to assess the overall quality of transported goods. As a result, this method cannot capture all the common irregularities that can occur during the delivery cycle. Therefore, an effective sensor solution to monitor such items is necessary.

Radio Frequency Identification (RFID) is a pragmatic wireless technology with a standardized communication protocol. Thus far, passive RFID temperature sensors have 
been investigated. However, each design has a limitation from which a set of design guidelines for an improved sensor solution is developed. That is, the new sensor should: (a) be compact to be applicable on individual products, (b) utilize purely passive technology to ensure longevity and cost-effectiveness, (c) monitor goods in a continuous fashion (e.g., operate through multiple room-to-cold and cold-to-room temperature cycles), and (d) operate in an independent mode, so that no resetting is required.

In this research, antenna systems and RF circuit design techniques are combined with Liquid Crystal Elastomers (LCEs) to develop three novel temperature sensors. LCEs are temperature responsive polymers that are programmable and reversible. Notably, LCEs return to their original state when the stimulus is removed. Also, for the first time, coldresponsive LCEs are incorporated into the designs presented in this research. Two of the developed sensors convey temperature changes through the controlled shift in the operating frequency. The third design conveys temperature threshold crossings by reversibly switching operation between two RFID ICs (or two Electronic Product Codes). Finally, all designs have been fabricated and tested with favorable results in accordance to the above mentioned guidelines. 


\section{TABLE OF CONTENTS}

CHAPTER

PAGE

\section{Contents}

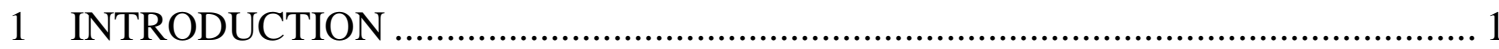

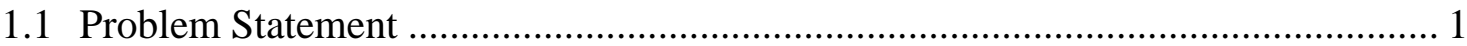

1.2 Research Objectives and Contributions ........................................................ 2

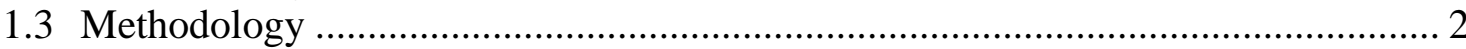

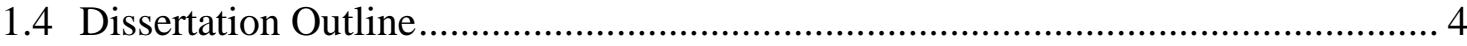

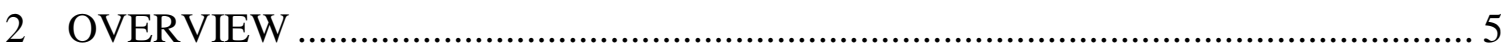

2.1 The Cold-Supply Chain (CSC) ...................................................................... 5

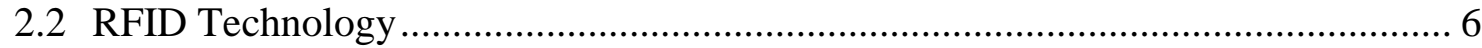

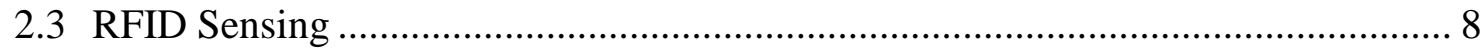

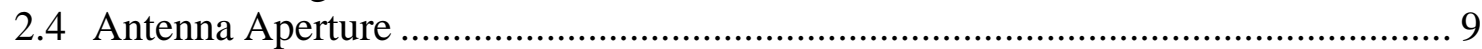

2.5 Backscattering Modulation............................................................................. 12

2.6 Friis Transmission Equation and the Power Transmission Coefficient (PTC) ...... 16

2.7 Matching Network Topologies for RFID Antenna Matching ............................. 18

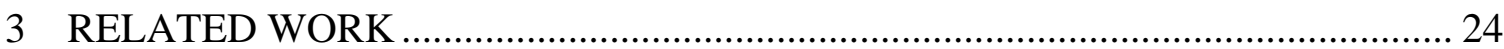

3.1 Commercially Available Temperature Sensors for the CSC.............................. 24

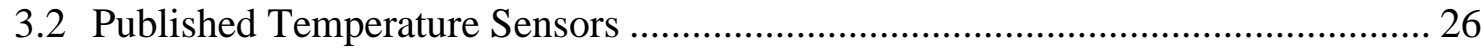

3.3 Summary of Available Temperature Sensing Technologies in the CSC .............. 29

4 LIQUID CRYSTAL ELASTOMERS (LCES) ................................................... 30

4.1 Liquid Crystals, Polymers, and Elastomers ....................................................... 30

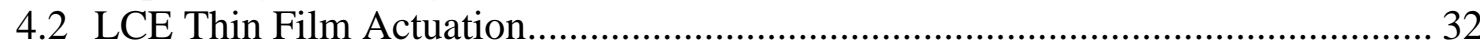

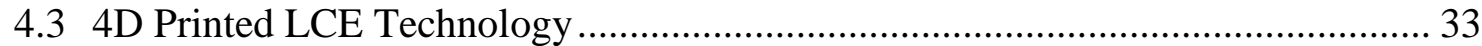

4.4 Cold Reactive Liquid Crystal Elastomers ......................................................... 37

4.5 Summary of Shape-Memory Materials ........................................................... 39

5 FREQUENCY SHIFTING ACTUATING DIPOLE ANTENNA DESIGN ............. 43

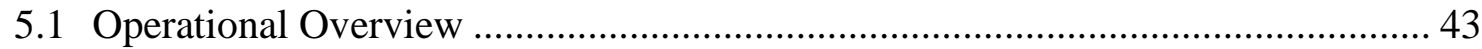

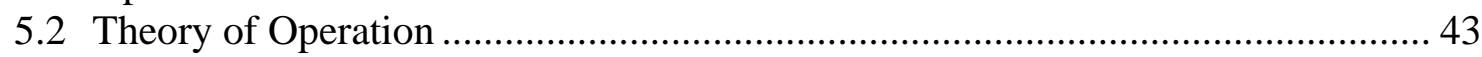

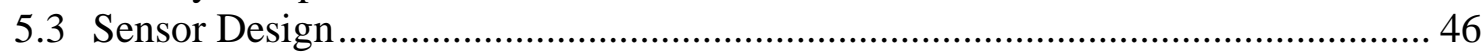

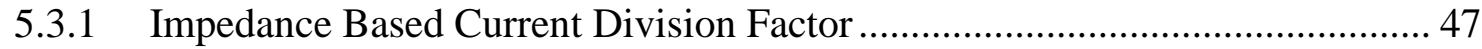

5.3.2 Geometry Based Current Division Factor.................................................. 49

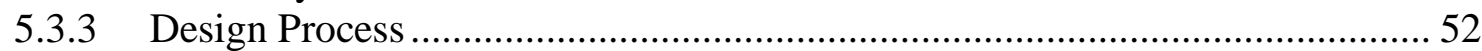

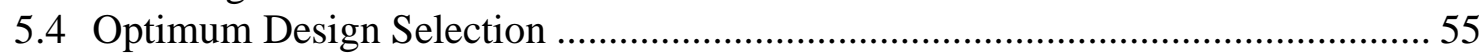

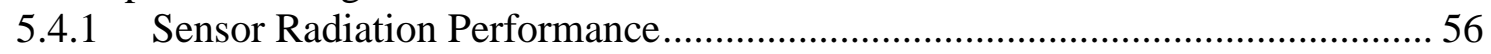

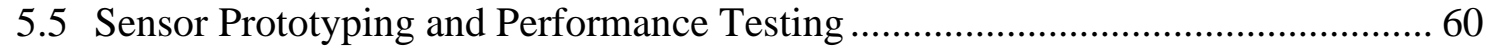


5.6 Final RFID passive Temperature Sensor............................................................... 68

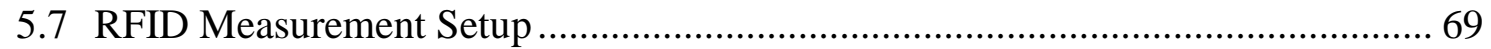

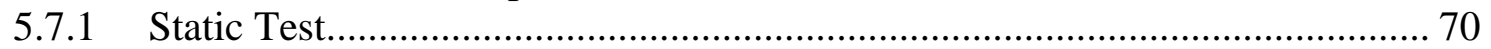

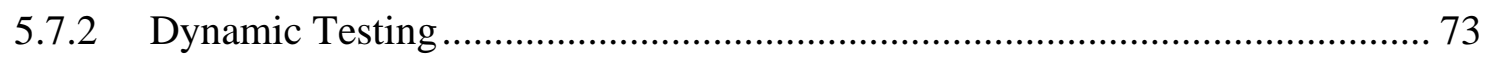

5.8 Simulation Modeling for Practical Applications .................................................... 76

5.8.1 Testing with Dual Sensors with Varying Orientation....................................... 79

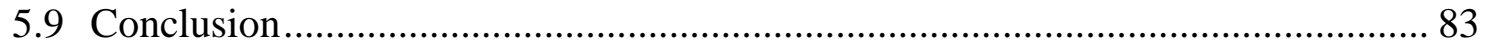

6 FREQUENCY SWITCHING PATCH ANTENNA DESIGN …………………......... 84

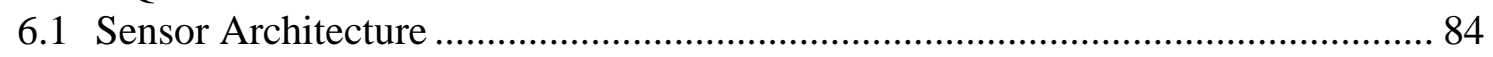

6.2 Patch Antenna Design with Passive Frequency Switching Capability ................... 85

6.2.1 Patch Antenna Theory ................................................................................. 86

6.2.2 Integrated Slot Design for Frequency Switching ............................................ 89

6.2.3 Passive Sensor Design with the USMS …………....................................... 92

6.2.4 Co-Simulation Setup and Antenna Design ...................................................... 93

6.3 Integration of Antenna and LCE Actuator ......................................................... 100

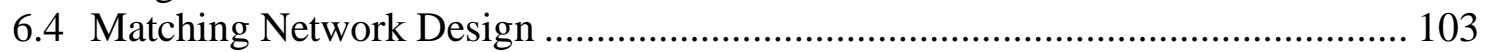

6.4.1 First Stage of the Matching Network: Shifting the Switching Bandwidth ..... 104

6.4.2 Second Stage of the Matching Network: Matching to the RFID IC ............... 108

6.4.3 Final Impedance Matching Network Design and Optimization ..................... 109

6.4.4 Impedance Matching Network Fabrication and Measurements ..................... 112

6.5 Final Design and RFID Performance Testing ................................................. 116

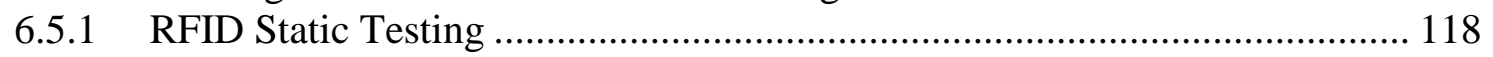

6.5.2 Frequency Shifting Using the LCE Array …….......................................... 121

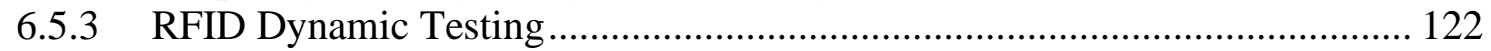

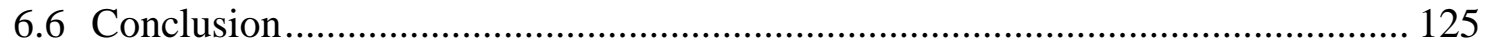

7 IC (EPC) SWITCHING BOW-TIE ANTENNA DESIGN …………..................... 127

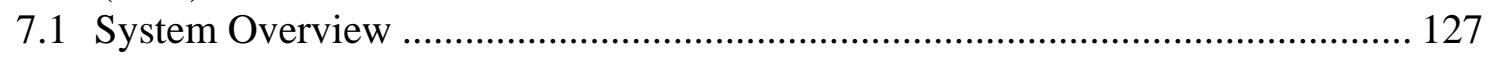

7.1.1 Antenna and Feed Structure ...................................................................... 128

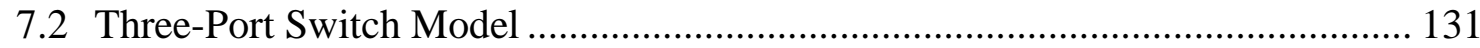

7.3 Matching Network Design ................................................................................. 133

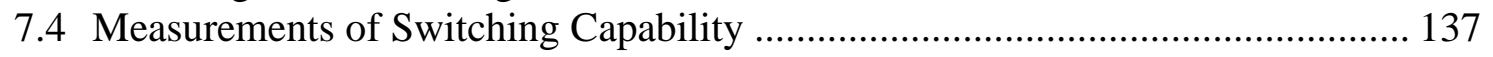

7.5 Validation of Temperature Sensor …………................................................. 142

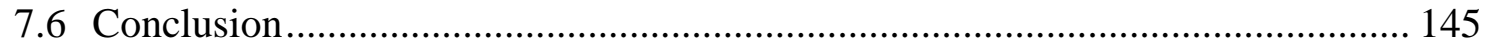

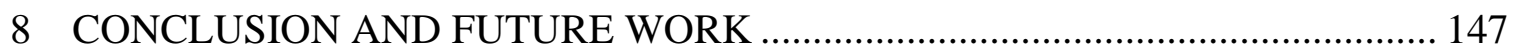

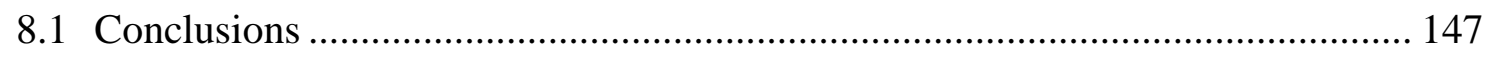

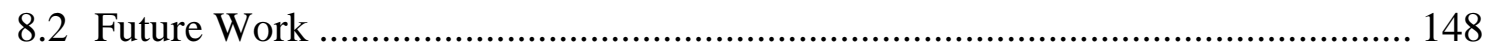

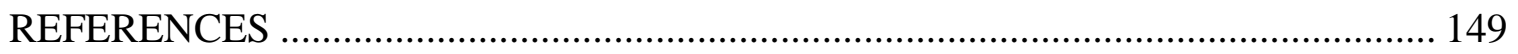

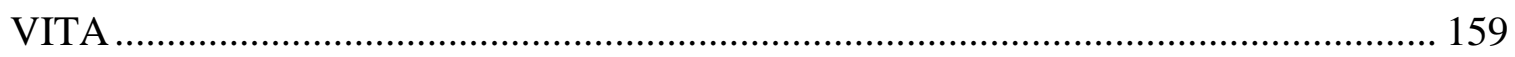




\section{LIST OF TABLES}

TABLE

PAGE

Table 3.1 Summary of advantages and disadvantages of the available RFID temperature sensor technologies.

Table 4.1 Comparison of Shape-Morphing Materials ............................................ 42

Table 5.1 Final design dimensions with the heights that correspond to operating

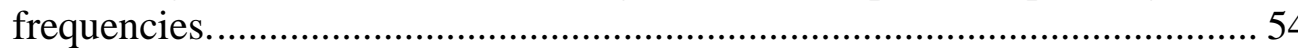




\section{LIST OF FIGURES}

FIGURE

PAGE

Figure 2.1. The Supply-Chain is complex and consists of many stages. ...................... 6

Figure 2.2. RFID operational block-diagram.................................................. 8

Figure 2.3. Impinging radiation density on antenna model..................................... 9

Figure 2.4. Equivalent circuit model of Antenna and Load model. ............................ 10

Figure 2.5. Model describing the antenna scattering aperture. ................................. 11

Figure 2.6. Conjugate matching achieved through matching network. ...................... 12

Figure 2.7. Backscatter modulation is achieved through modulating the load

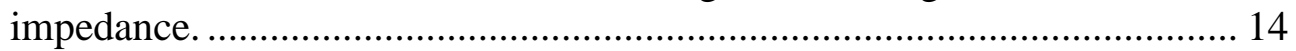

Figure 2.8. Backscatter modulation analogy using a reflective surface...................... 14

Figure 2.9. $\quad$ RFID reader and tag setup with modulated EPC based backscattered

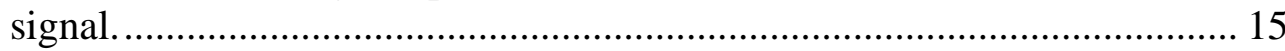

Figure 2.10. Simplified diagram of the incident and reflected wave that may cause

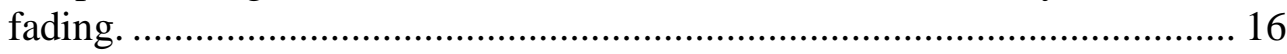

Figure 2.11. RFID antenna terminated with RFID IC impedance. .............................. 17

Figure 2.12. T-match and inductively coupled loop matching network topologies......... 19

Figure 2.13. Formation of a folded dipole antenna............................................... 19

Figure 2.14. Transformer model of the folded dipole antenna..................................... 20

Figure 2.15. Customizable input impedance due to the modified inner arm geometry... 21

Figure 2.16. Transformer model of the T-matching network. ..................................... 22

Figure 2.17. The input impedance is customizable by adjusting the dimensions $a, b, w$,

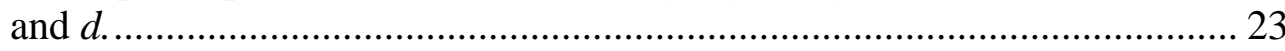

Figure 2.18. Transformer model for the inductively coupled loop matching network. ... 23

Figure 4.1. Cross-linked LC polymer chains aligned in the ordered state.................... 31 
Figure 4.2. Actuation due to the LC polymer transition from the nematic phase to the isotropic phase when stimulated.

Figure 4.3. Hierarchical neamatic vector to obtain various twist and curves. 33

Figure 4.4. Molecular alignment of the LCE polymer chains coincides with the print path.

Figure 4.5. Azimuthally varying nematic vector of the Archimedean spiral design. .... 35

Figure 4.6. Fabricated 4D printed Archimedean spiral design in the stimulated state. . 36

Figure 4.7. Heat reactive 4D printed LCE array: (a) relaxed state (b) excited state...... 37

Figure 4.8. Cold-reactive LCE cell with radial alignment. ...................................... 38

Figure 4.9. Array of cold-reactive LCE cells (left). Array in the relaxed (right upper) and excited states (right lower)............................................................. 38

Figure 4.10. Actuation displacement comparison. (left) $1.23 \mathrm{~mm}$ for cold-reactive LCE and (right) $4 \mathrm{~mm}$ for hot-reactive LCE............................................... 39

Figure 5.1. Frequency shifting capability of the developed sensor design. ................. 43

Figure 5.2. The operational frequency of the sensor is controlled through the actuation of the designed antenna above its ground plane using LCEs. ................... 44

Figure 5.3. Planar dipole antenna with the ITMN ................................................ 46

Figure 5.4. A secondary branch of conductors is added to an unmatched dipole to achieve an impedance match at the input port by adjusting the dimensions $x, l^{\prime}$, and $a^{\prime}$.

Figure 5.5. Electrical equivalent radius (EER) of a flat planar conductor. 49

Figure 5.6. Planar antenna design with the ITMN and the EERs defined for the flat conductors.

Figure 5.7. ANSYS HFSS simulation model of the planar dipole antenna design on FR4 substrate over the ground plane.

Figure 5.8. Current division factor, $\alpha$, for different dipole lengths and widths. 53

Figure 5.9. ANSYS HFSS simulation model of the planar antenna design with the ITMN. 
Figure 5.10. Power transmission coefficient vs. frequency for designs A, B, and C, from Table. IV. This plot depicts the performance of each design at the two operating frequencies of $902 \mathrm{MHz}$ and $928 \mathrm{MHz}$.

Figure 5.11. Simulated 3D radiation pattern of the designed sensor. The radiation pattern and maximum gain are similar for both cases when the antenna is elevated above the ground plane to $3.6 \mathrm{~mm}$ (operating at $902 \mathrm{MHz}$ ) and when the antenna is elevated to $6.8 \mathrm{~mm}$ above the ground plane (operating at $928 \mathrm{MHz}$ ).

Figure 5.12. HFSS polar gain plot for the XY principal plane. ................................... 58

Figure 5.13. HFSS polar gain plot for the YZ principal plane.................................... 58

Figure 5.14. HFSS polar gain plot for the XZ principal plane.................................... 59

Figure 5.15. Prototype of planar antenna design with ITMN. .................................... 60

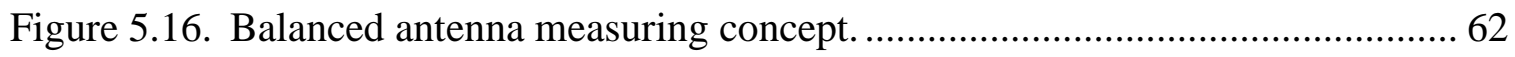

Figure 5.17. Fabricated differential probe for balanced antenna impedance measurements.

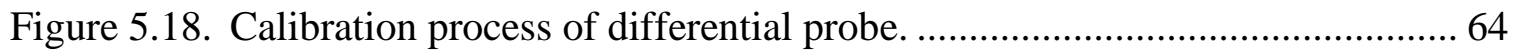

Figure 5.19. Impedance measurement setup with differential probe and VNA..............65

Figure 5.20. Measured and simulated input impedance of the antenna at $3.6 \mathrm{~mm}$ above the ground plane, which corresponds to an operating frequency of 902 $\mathrm{MHz}$.

Figure 5.21. Measured and simulated input impedance of the antenna at $6.8 \mathrm{~mm}$ above the ground plane, which corresponds to an operating frequency of 928 $\mathrm{MHz}$.

Figure 5.22. Measured and simulated antenna PTCs.

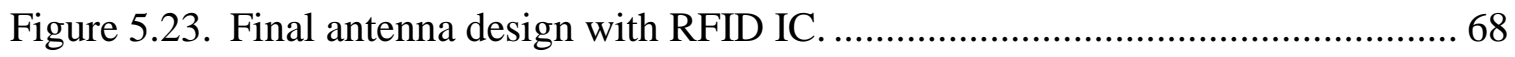

Figure 5.24. Final sensor prototype with ground plane on a heat source....................... 68

Figure 5.25. RFID measurement setup with vertical polarization orientation................ 69

Figure 5.26. Static test setup of the proposed RFID tag. ......................................... 71

Figure 5.27. Static measurements of the RFID tag at two different heights.................. 72

Figure 5.28. Dynamic test setup of the proposed passive RFID temperature sensor....... 73 
Figure 5.29. Dynamic RFID test setup with hot-plate as heat source to stimulate the LCE array. (a) The LCE actuates the antenna by $4 \mathrm{~mm}$ at $160^{\circ} \mathrm{C}$. (b) Once the heat source is turned off, the LCE automatically returns to a flat-relaxed state...................................................................................... 74

Figure 5.30. Dynamic measurements of the RFID tag at two different heights. ............. 75

Figure 5.31. Simulation model of the shipping box modeled with various material properties and with a payload of fresh water.

Figure 5.32. PTC simulation results of the developed sensor when placed on a shipping box made from various materials.

Figure 5.33. Simulation model of the DSUT in the presence of an identical sensor with varying orientations.

Figure 5.34. Simulated performance of our sensor at room and elevated temperatures when it placed near an identical sensor with varying.

Figure 6.1. Patch antenna model. 86

Figure 6.2. The dominant mode of the patch antenna has a TM10z field profile 87

Figure 6.3. Surface current distribution for the TM10z mode. 88

Figure 6.4. The voltage, current, and impedance profile over the length of the patch for the dominant mode.

Figure 6.5. When a slot is added to a metallic patch, the current has a longer path to travel which increases the length of the patch (from $L$ to $L^{\prime}$ ) and decreases the resonant frequency as per (6.5).

Figure 6.6. Simulated $\mathrm{S}_{11}$ of a patch antenna with and without a slot.

Figure 6.7. The USMS is connected across the slot and provides the passive frequency switching capability of the antenna when it is activated and deactivated. .. 93

Figure 6.8. The simulated patch antenna is converted to a two-port system by adding an additional port at the slot, where we intend to connect the switch.

Figure 6.9. A parametric sweep of the slot width, length, and position is conducted using HFSS.

Figure 6.10. Microstrip test fixture for connecting and measuring the switch. (Top) Fixture without switch for phase compensation. (Bottom) Fixture with switch. 
Figure 6.11. Measured insertion loss of the switch at its ON and OFF states. 96

Figure 6.12. ANSYS Circuit Designer setup for connecting the measured switch data to the antenna.

Figure 6.13. Effect of the operating frequency of the proposed antenna with the switch activated as a function of slot position, length, and width

Figure 6.14. Effect of the operating frequency of the proposed antenna with the switch deactivated as a function of slot position, length, and width. 98

Figure 6.15. Simulated $S_{11}$ for the selected antenna design. 99

Figure 6.16. Fabricated antenna with the switch soldered across its slot 100

Figure 6.17. 3D printed frame that translates the actuation of the LCE array. a) The LCE is at its relaxed state and the switch is set at its OFF (de-pressed or deactivated) state. (b) The LCE is actuated and the switch is set to its ON (pressed or activated) state. 101

Figure 6.18. VNA test setup and fabricated antenna.............................................. 102

Figure 6.19. Measured and simulated results of RFID antenna with switch. ............... 103

Figure 6.20. Measured input impedance of the fabricated antenna with the impedances at the current operating frequencies labelled.

Figure 6.21. Measured input impedances of the fabricated antenna with the impedances at the desired operating frequencies labelled. 106

Figure 6.22. First stage of matching network. The input of $1.209+\mathrm{j} 8.192 \Omega$ at 902 and $927 \mathrm{MHz}$ is translated to $50 \Omega$ at 906.1 and $923.9 \mathrm{MHz}$, respectively (refer to Figure 6.23).

Figure 6.23. Simulated response of the output of the $1^{\text {st }}$ stage of the matching network.

Figure 6.24. Second-stage of matching network. The input of $50 \Omega$ at 906.1 and 923.9 $\mathrm{MHz}$ (refer to Figure 6.23) is translated to the conjugate input impedance of the RFID IC at 902.2 and $921.1 \mathrm{MHz}$, respectively. 108

Figure 6.25. First and second stages of the matching network combined. 109

Figure 6.26. Simulated PTC of antenna with the final matching network. 110

Figure 6.27. Final optimized and miniaturized matching network. 
Figure 6.28. Simulated PTC of antenna with final matching network. The final operating frequencies are 921.1 MHz and 902.2 MHz at the OFF (deactivated or de-pressed) and ON states of the switch (activated or pressed), respectively.

Figure 6.29. Two versions of the matching network are fabricated. (Left) An IPX port is connected for VNA measurement testing. (Right) An RFID IC is connected for RFID testing.

Figure 6.30. VNA setup with custom calibration standards for the IPX to SMA cable extension.

Figure 6.31. The impedance matching network with the IPX port is connected to the antenna. The performance of the design is tested using the VNA

Figure 6.32. Measured and simulated PTC of the antenna with matching network. When the switch is OFF (not pressed) the measured antenna operates at 922.1 MHz. When the switch is ON (pressed) the measured antenna operates at $904 \mathrm{MHz}$.

Figure 6.33. The measured input impedance of antenna with switch and matching network at both states of the switch.

Figure 6.34. Passive RFID temperature sensor.

Figure 6.35. Diagram of the RFID measurements setup

Figure 6.36. Static RFID testing.

Figure 6.37. Static RFID measurement results with the switch at the OFF (deactivated) state.

Figure 6.38. Static RFID measurement result with the switch in the ON (activated) state.

Figure 6.39. The LCE array is relaxed at $29^{\circ} \mathrm{C}$. The operating frequency of the antenna design is $910 \mathrm{MHz}$.

Figure 6.40. The LCE array is actuated at $-9^{\circ} \mathrm{C}$. The switch has been triggered and the operating frequency of the antenna design is $882 \mathrm{MHz}$......................... 122

Figure 6.41. Dynamic RFID setup with the designed sensor at $-17^{\circ} \mathrm{C}$. ..................... 123

Figure 6.42. Dynamic RFID measurement results when the switch in the ON (triggered) state.

Figure 6.43. Dynamic RFID setup with the designed sensor at $28^{\circ} \mathrm{C}$. 
Figure 6.44. Dynamic RFID measurement results with the switch OFF (deactivated) state.

Figure 7.1. Operational diagram of the proposed temperature sensor. ...................... 127

Figure 7.2. Bow-tie antenna design with feed network.......................................... 129

Figure 7.3. Antenna radiation pattern (realized gain in $\mathrm{dBi}$ ) showing $360^{\circ}$ coverage in the elevation-plane.................................................................... 130

Figure 7.4. VNA test setup with fabricated antenna. .......................................... 130

Figure 7.5. Measured and simulated S11 of the proposed antenna.......................... 131

Figure 7.6. Mechanical switch testing circuit. (a) Mechanical switch with deembedding circuit. (b) De-embedding circuit for electrical length compensation.

Figure 7.7. Insertion losses of the 3-port mechanical switch for the deactivated and activated states.

Figure 7.8. Antenna and mechanical switch model in ANSYS Circuit Designer....... 134

Figure 7.9. Input impedance at port 2 for switch in activated and deactivated states.. 135

Figure 7.10. Input impedance at port 3 for switch in activated and deactivated states.. 136

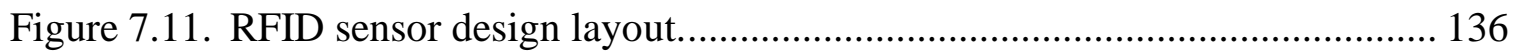

Figure 7.12. Simulated PTC at the ports where RFID ICs 1 and 2 will be connected, as shown in Figure 7.11................................................................... 137

Figure 7.13. Isolation between RFID IC 1 port and RFID IC 2 port. .......................... 138

Figure 7.14. Test setup with fabricated prototype for impedance measurements at the ports where the RFID ICs are connected........................................... 138

Figure 7.15. Simulated and measured input impedances at RFID IC 1 port................. 139

Figure 7.16. Simulated and measured PTC at RFID IC port 1................................ 140

Figure 7.17. Simulated and measured PTC at RFID IC port 2............................... 141

Figure 7.18. Simulated and measured PTC at RFID IC port 2............................... 141

Figure 7.19. RFID temperature sensor with cold-responsive LCE array..................... 142 
Figure 7.20. RFID temperature sensor with RFID reader inside freezer at roomtemperature. ............................................................................ 143

Figure 7.21. Only RFID IC 1 is read at room temperature. ....................................... 144

Figure 7.22. RFID temperature sensor with RFID reader inside freezer at $-15^{\circ} \mathrm{C} \ldots \ldots . .144$

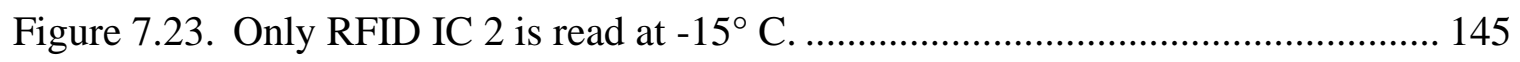




\section{CHAPTER 1}

\section{INTRODUCTION}

\subsection{Problem Statement}

The Cold-Supply-Chain (CSC) involves the delivery of fresh-food and pharmaceutical products that must be maintained within a controlled temperature environment from the manufacturer to the end-user. Hence, the CSC ensures the safety and quality of fresh-food products [1]. Furthermore, the CSC is important since $65 \%$ of the retail food market currently consists of fresh-food products [2]. Presently, temperature monitoring is conducted at three locations within the delivery cycle from the supplier to the consumer. Specifically, at the sending and receiving checkpoints and at the transportation containers [3]. Furthermore, the temperature conditions recorded from these locations are then used to assess the overall condition of the perishable products [3]. Consequently, this methodology of monitoring the quality of fresh-food products fails to account for the temperature conditions due to common anomalies that occur in the CSC (discussed in detail in Section 2.1) [2]-[5]. As a result, a fresh-food product could be exposed to damaging temperature conditions without the knowledge of the consumer.

Moreover, in the CSC, 30\% of fresh-food products are damaged during transit [3]. Additionally, in 2015, the global sales of organic food-products resulted to approximately $\$ 81.6$ billion [5] and the total sales of biopharmaceuticals totaled to nearly $\$ 260$ Billion [20]. The currently available technology for monitoring perishable food products are limited in one way or another. In summary, they require additional specialized software, are costly, require frequent battery changes, or do not provide real-time readings. Finally, due to the safety-risks, high consumption rates of fresh-food products, financial-risks 
involved with transporting perishable goods, and drawbacks related to currently available sensor technologies, the designs of effective temperature sensors, which can provide continuous end-to-end monitoring in the CSC are conveyed in this research.

\subsection{Research Objectives and Contributions}

To design temperature sensors to provide end-to-end monitoring in the CSC and resolve the limitations in the currently available sensor technologies, the design of any new temperature sensor must concurrently have the following capabilities: (a) operate through purely passive technology (i.e., battery-less) in order to ensure operational longevity and cost-effectiveness, (b) monitor individual items or crates (i.e., operate wirelessly), (c) function in a continuous temperature monitoring mode (e.g., able to detect multiple cycles of hot-to-cold and cold-to-hot temperature variations), (d) operate without requiring any form of resetting (i.e., operate autonomously), and (e) have a compact form-factor so that it can be attached on items with various sizes in the CSC. In this research, three novel temperature sensors with these features are developed, designed, fabricated, and tested.

\section{$1.3 \quad$ Methodology}

The sensors designed in this research operate using the Radio Frequency Identification (RFID) platform. RFID technology consists of a wireless network of readers (or interrogators) and transponder tags that communicate through a standardized and robust communication protocol that can address all the desired attributes (mentioned in Section 1.2) of the new sensors designed for the CSC in this research [6, pp. 141-144], [7, pp. 4246]. Specifically, this technology communicates (or transfers data) through a purely passive methodology, where the incident signal from the reader is returned (or reflected) back in a 
modulated form by the tag, this technique is called backscattering modulation. In addition, each RFID tag consists of a unique Electronic Product Code (EPC) [6, p. 366], [8]. Accordingly, backscattering modulation is based on this code. Therefore, due to these functionalities, RFID is a favorable technology to support novel sensor designs since it is immune to environments with high clutter or large amounts of electromagnetic (EM) wave reflections from nearby objects. Also, RFID technology is proficient in operating with multiple RFID sensors in close-proximity (i.e., dense network environment) [7, pp. 7-9]. Moreover, when reading an RFID tag, RFID readers provide information on the following parameters: the frequency of operation, the EPC, and the transmit and receive power levels. Hence, these parameters serve as markers through which sensing can be performed.

For the first time, the RFID temperature sensors designed here will utilize Liquid Crystal Elastomers (LCEs) to achieve continuous, autonomous, and battery-free functionality. LCEs are shape-morphing stimuli-responsive polymers that are programmable and reversible [9]-[14]. Temperature responsive LCEs are strategically incorporated into antenna designs to vary one of the above-mentioned parameters (i.e., frequency, tag ID, or receive/transmit power level) in a controlled manner. Also, ANSYS HFSS and ANSYS Circuit Designer are the software packages used for the design of the proposed sensors.

In summary, due to the inherent operation of RFID communications (i.e., backscattering modulation of the EPC) and the incorporation of LCE technology, the proposed sensors will achieve all the desired properties that were described in Section 1.2 (i.e., they are purely passive, and they operate continuously and autonomously). Finally, using antenna miniaturization techniques, the size of the proposed sensors is reduced. 


\subsection{Dissertation Outline}

The overview in chapter 2 presents important introductory information that supports the work done throughout this research; that is, the details pertaining to the Cold Supply Chain (CSC) and important technical features related to RFID technology. A comparative analysis of the available technologies for monitoring perishable items in the CSC, whose cumulative limitations provide the framework of our design objectives, is

discussed in chapter 3. In chapter 4, the theory of Liquid Crystal Elastomer (LCE) technology and the relevant 4D printed LCE designs are discussed.

Additionally, chapters 5, 6, and 7 detail the novel temperature sensors developed by this research. Specifically, the proposed designs consist of a frequency-shifting dipole antenna design, a frequency-switching patch antenna design, and a IC switching bow-tie antenna design. Finally, the conclusions for our designs and the future work regarding LCE integrated sensors are discussed in chapter 8. 


\section{CHAPTER 2}

\section{OVERVIEW}

\subsection{The Cold-Supply Chain (CSC)}

A significant variety of essential organic-foods and pharmaceutical products must be maintained within a controlled climate to ensure a safe quality for human consumption [3], [15]-[18]. The Cold-Supply-Chain (CSC) is intended to provide such an environment during the delivery of perishable items [17]. As a result, the CSC ensures the longevity, safety, and quality of fresh-food products. Moreover, during the delivery phase, items must be maintained within a temperature range of $-1^{\circ}$ to $10^{\circ} \mathrm{C}$ to minimize metabolic and microbial deterioration or spoilage [1], [17], [18]. Conversely, when fresh-food products, such as, fruits, vegetables, and meats, are exposed to excessively high or low temperatures, they experience discoloration and loss of nutrients [17]. Additionally, medicinal products, such as, insulin, vaccines, medication, and blood-products, can lose potency [18].

Furthermore, the demand of the CSC is currently increasing. That is, due to increasing populations, developing nations, technology advancements, and supportive federal regulations, the CSC has become critical to the growth of global trade and to the worldwide availability of food and health supplies [19], [20]. As briefly mentioned in Section 1.1, common anomalies can occur along the delivery cycle in the CSC where temperature monitoring is overlooked [3], [4]. Additionally, the supply-chain is often complex, involving many stages from the crop-extraction to the end-user, refer to Figure 2.1. This complexity increases the probability of a fresh-food product being exposed to excessive temperatures due to various anomalies. Common anomalies that can happen are malfunctioning refrigeration equipment and extended wait-times at loading/unloading 
docks [21]. Moreover, operator errors in the form of carelessness to the regularity of opening or leaving the container doors open, road accidents, and failure to pre-cool the container prior to loading are complications that often occur. Finally, a major detail that is often overlooked by the operator is the improper placement of perishable items within the delivery vehicle and inadequate packaging, which both inhibit the products from being maintained at the proper temperature during the delivery cycle [17], [21]. These drawbacks cause vulnerabilities in the robustness of the CSC process. To address these problems in the CSC, novel passive temperature sensors using liquid crystal elastomers (LCEs), which operate autonomously and continuously with manufacturer-to-end-user coverage are designed in this research.

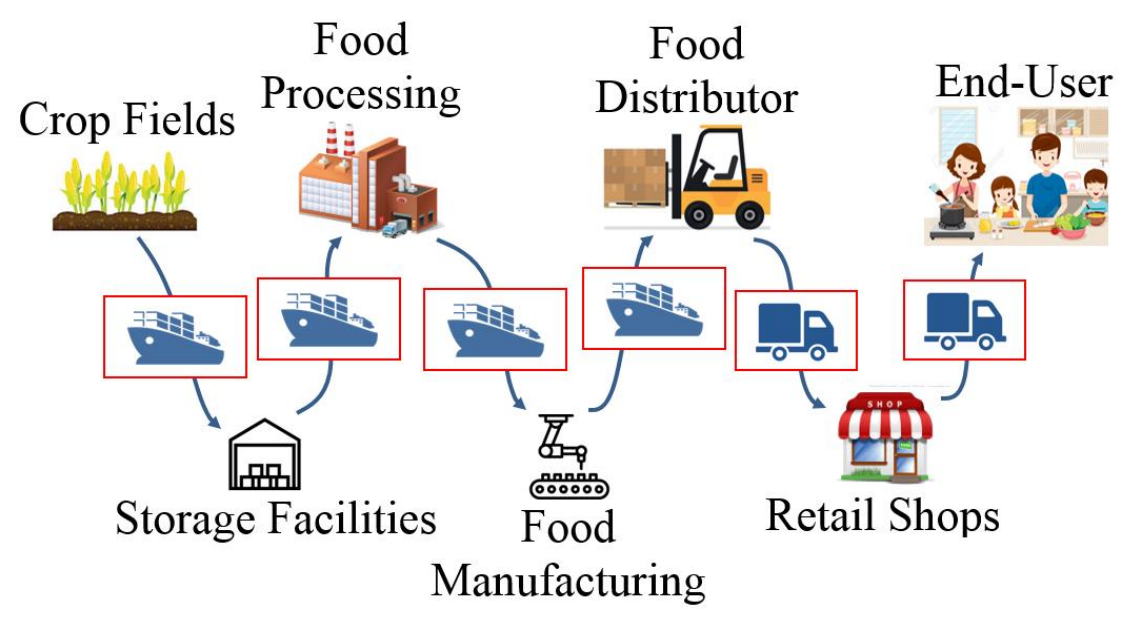

Figure 2.1. The Supply-Chain is complex and consists of many stages.

\subsection{RFID Technology}

Radio Frequency Identification (RFID) consists of transponder tags that transmit data wirelessly to track, identify, and detect people and objects when interrogated through a continuous wave (CW) electromagnetic (EM) signal. RFID technology consists of three topologies based on their power sources. They are passive, semi-passive, and active tag designs [7, pp. 34-42]. Passive tags operate purely through the incident EM wave. The 
RFID IC uses an internal rectifier to power itself and it such rectifiers typically provide efficiencies near $30 \%$ [7, p. 77]. Secondly, semi-passive tag designs consist of an on-board battery and utilize the incident EM interrogation field as a turn-on signal, which activates the battery and supplies power to the RFID IC. Both the passive and semi-passive tag designs are similar in that they communicate using backscattering modulation, which is discussed further in subsequent sections. Finally, the third design is the active design, which not only has an on-board battery but also has an entire transmit radio system. In summary, semi-passive and active tags use additional batteries and complex circuitry, which limit their longevity, cost-effectiveness and applicability to fast-paced and narrowmargin environments, such as, the Cold-Supply-Chain.

Furthermore, typical applications of RFID technology consist of conveyer-belt processing, warehouse inventory, consumer product safety, and security access control [7, pp. 15-22, 33, 44-46, 274-277]. Furthermore, RFID tags can be designed with various form-factors depending on the application. For example, certain tags are designed on flexible substrates to conform to various shapes of objects [7, pp. 37, 333, 354], [32, pp. 19-20], [22]. Additionally, RFID tags are miniaturized, on the scale of $1 \mathrm{~mm}$ in width and $10 \mathrm{~mm}$ in length, for implanting into animals and humans [23], [32, pp. 364-371], [24]. Finally, RFID tags intended for human implantation are useful for applications, such as, wireless payments and security access and can also be used to store personal health record information in emergency situations [23]. 


\subsection{RFID Sensing}

The functionalities of RFID technology are summarized in the flow chart of Figure 2.2 .

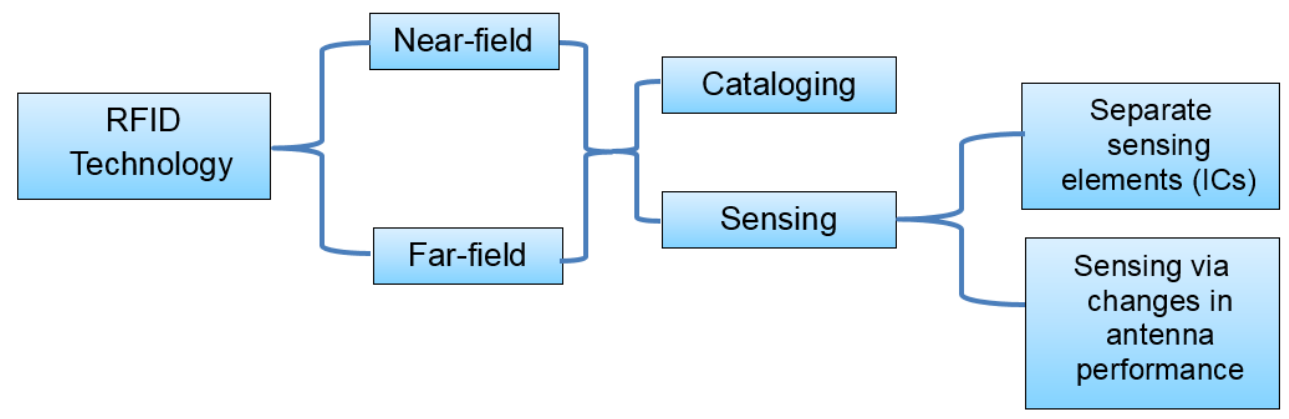

Figure 2.2. RFID operational block-diagram.

Accordingly, RFID systems can operate in the near-field. However, this method is limited to close-proximity applications [7, pp. 11-12, 284-286], [32, pp. 26-27, 30-33, 68-70]. Alternatively, RFID can operate in the far-field using radiated EM fields, which is preferred in applications that require longer range [7, p. 28, 111-114], [32, pp. 26-27]. Moreover, as described in Section 2.2, RFID was originally designed for tasks related to cataloging and identification [7, pp. 15-22]. However, in recent years, RFID technology has evolved into sensing applications [8], [25], [26], [27]. Sensing through RFID technology can be accomplished through two methods.

The first method which is the most common, consists of using separate on-board sensors in the form of integrated circuits (ICs) that perform the sensing function. The analog data is digitized and down-linked to the reader. Unfortunately, this type of RFID sensor topology comes with a variety of inconveniences. Specifically, proprietary software is required to decode and process the digitized data, limited-operational usage due to an active system design (e.g., requires batteries), and high costs related to the additional components and required maintenance [28]-[31]. 
The second method of sensing using RFID technology is accomplished by designing antennas that change their performance in response to changes in the environment. This technique has the advantage of providing purely passive (i.e., batteryfree) sensors [8], [25]-[27]. Temperature responsive liquid crystal elastomers (LCEs) are the sensing elements, which will be integrated into our RFID antenna structures to detect temperature changes. Finally, these changes in the antenna performance will be recorded through the RFID communication platform.

\subsection{Antenna Aperture}

Consider an antenna defined as a complex impedance $\mathrm{Z}_{\mathrm{A}}=\mathrm{R}_{\mathrm{A}}+\mathrm{j} \mathrm{X}_{\mathrm{A}}$ connected to complex load $\mathrm{Z}_{\mathrm{L}}=\mathrm{R}_{\mathrm{L}}+\mathrm{j} \mathrm{X}_{\mathrm{L}}$ with an incident wave consisting of radiation density (S) impinging on the antenna. This impinging wave results in an induced power $\left(\mathrm{P}_{\mathrm{RX}}\right)$ at the terminals of the antenna, this model is depicted in Figure 2.3.

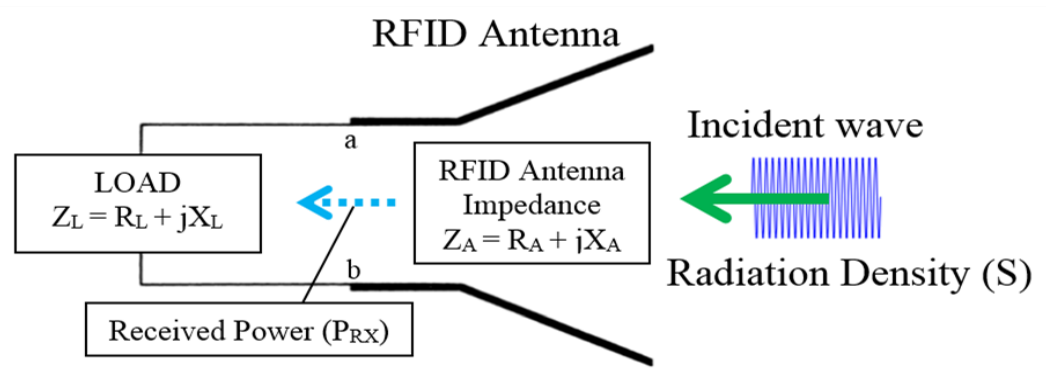

Figure 2.3. Impinging radiation density on antenna model.

The real part of the antenna complex impedance is defined as the radiation resistance $\left(\mathrm{R}_{\mathrm{r}}\right)$ where $R_{A}=R_{r}$ in the model. This resistance represents the capability of the antenna to radiate energy in the transmitting mode and is responsible of the transfer of energy from the free-space wave to the antenna in the receiving mode [32, pp.121-124], [33, pp. 3, 8085]. 
Additionally, due to the incident radiation density, a voltage or equivalently a current is induced within the antenna, refer to Figure 2.4. This current then flows through the remaining circuit including the radiation resistance $\left(\mathrm{R}_{\mathrm{r}}\right)$. As a result, the power corresponding to the radiation resistance $\left(P_{S}=I^{2} R_{r}\right)$ is re-scattered/re-radiated into freespace [32, pp.121-124], [33, pp. 80-85]. The equivalent circuit model describing this notion is shown in Figure 2.5.

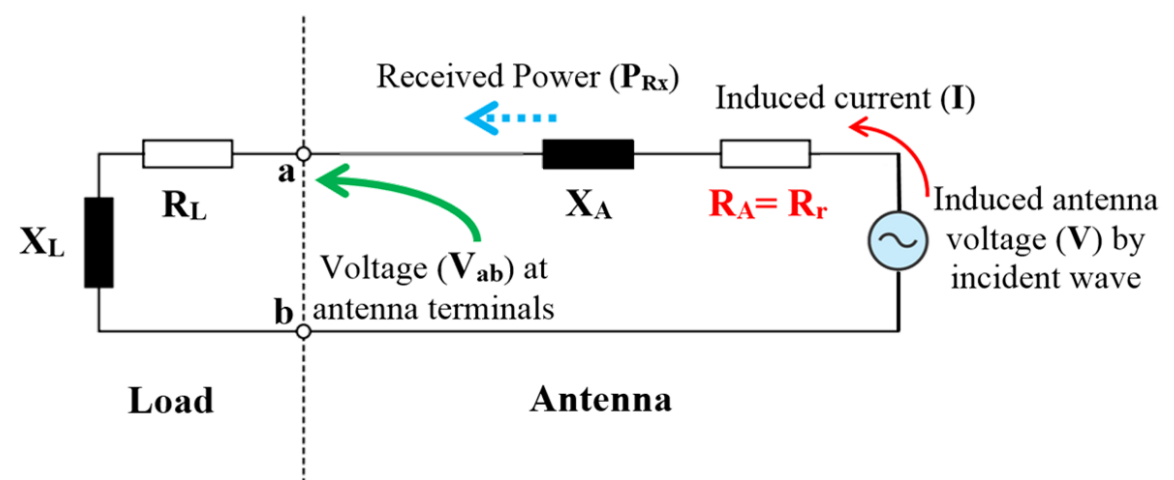

Figure 2.4. Equivalent circuit model of Antenna and Load model.

The effective aperture $\left(A_{e}\right)$ of a receiving antenna represents its ability to capture power from the power flux density of an incident wave (S) and pass the power ( $\left.\mathrm{P}_{\mathrm{RX}}\right)$ to the load [33, p. 91]. Furthermore, according to Figure 2.3, the effective aperture is the ratio of the power received at the terminals of the antenna $\left(\mathrm{P}_{\mathrm{RX}}\right)$ to the incident radiation density (S) or $A_{e}=P_{R X} / S$ [32, pp.121-124]. In general, the maximum effective aperture $\left(\mathrm{A}_{\mathrm{e} \_ \text {max }}\right)$ of any antenna is related to its gain in the following fashion [32, p. 124], [33, p. 91]:

$$
A_{e_{-} \max }=\frac{\lambda^{2}}{4 \pi} G
$$

In the opposite sense, the scattering aperture is the ability of the antenna to pass the power $\left(\mathrm{P}_{\mathrm{S}}\right)$ through the scattering aperture $\left(\mathrm{A}_{\mathrm{S}}\right)$ to emit a radiation density $(\mathrm{S})$. It is defined as the 
following ratio: $P_{S} / S$ [32, pp.121-124]. A model describing this process is depicted in Figure 2.5.

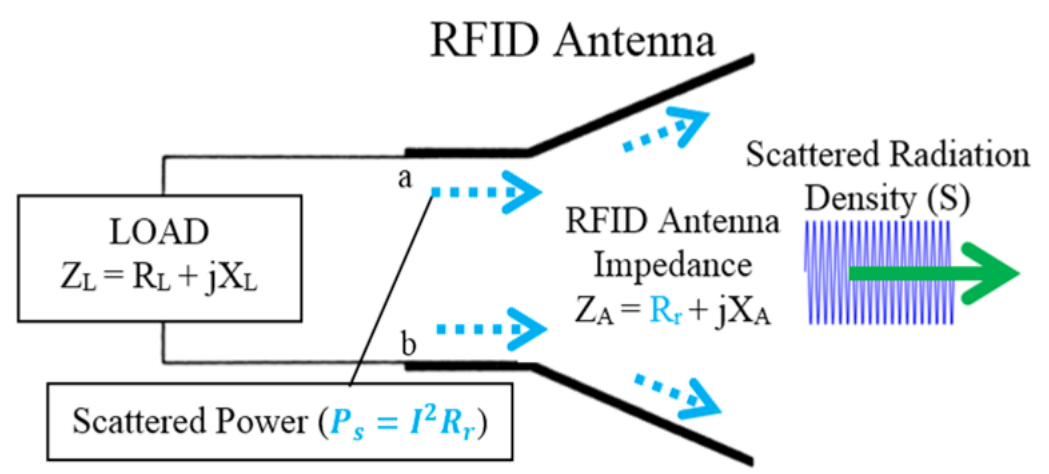

Figure 2.5. Model describing the antenna scattering aperture.

Typically, the scattering aperture is also often referred to as the Radar Cross Section ( $\sigma$ ) of the antenna [32, p. 123]. In reference to Figures 2.4 and 2.5, the scattering aperture of an antenna with radiation resistance $\mathrm{R}_{\mathrm{r}}$ can be found in the following way:

$$
\sigma=A_{S}=\frac{P_{S}}{S}=\frac{I^{2} R_{r}}{S}
$$

Moreover, the current $I$ from Figure 2.4 is:

$$
I=\frac{V}{Z_{A}+Z_{L}}=\left.\frac{V}{\sqrt{\left(R_{A}+R_{L}\right)^{2}+\left(X_{A}+X_{L}\right)^{2}}}\right|_{R_{A}=R_{r}}
$$

Substituting (2.3) into (2.2) give the following equation for the scattering aperture:

$$
\sigma=A_{S}=\frac{P_{S}}{S}=\frac{I^{2} R_{r}}{S}=\left.\frac{V^{2} R_{r}}{S\left[\left(R_{A}+R_{L}\right)^{2}+\left(X_{A}+X_{L}\right)^{2}\right]}\right|_{R_{A}=R_{r}}
$$

Equation (2.4) is in reference to the power being dissipated (in the form of re-scattered power, $\mathrm{P}_{\mathrm{s}}$ ) through the radiation resistance $\mathrm{R}_{\mathrm{r}}$. In a similar fashion, the effective aperture can be written in terms of the received power (which is dissipated at the load) as follows:

$$
A_{e}=\frac{P_{R X}}{S}=\frac{I^{2} R_{L}}{S}=\left.\frac{V^{2} R_{L}}{S\left[\left(R_{A}+R_{L}\right)^{2}+\left(X_{A}+X_{L}\right)^{2}\right]}\right|_{R_{A}=R_{r}}
$$


Furthermore, considering equations (2.4) and (2.5), in a conjugate matched condition $\left(Z_{L}=Z_{A}^{*}\right)$ the scattering and effective apertures of an antenna are equal [32, p. 123], [34, pp. 29-35]:

$$
\sigma=A_{S}=A_{e}=\left.\frac{V^{2}}{4 S R_{A}}\right|_{R_{A}=R_{r}}
$$

This implies that from the total power of the incident radiation density $(\mathrm{S})$ captured by the antenna, half is dissipated into the load $\left(\mathrm{P}_{\mathrm{RX}}\right)$ and the remaining half is re-radiated back into free-space $\left(\mathrm{P}_{\mathrm{S}}\right)$ [32, p. 123], [33, p. 85]. There exists no other combination of matching (aside from conjugate matching), which will deliver more power to the load; hence, (2.6) also serves as another form of the equation for the maximum effective aperture of the antenna. Additionally, as depicted in Figure 2.6, a matching network is typically utilized to establish the conjugate matching between the antenna and the load.

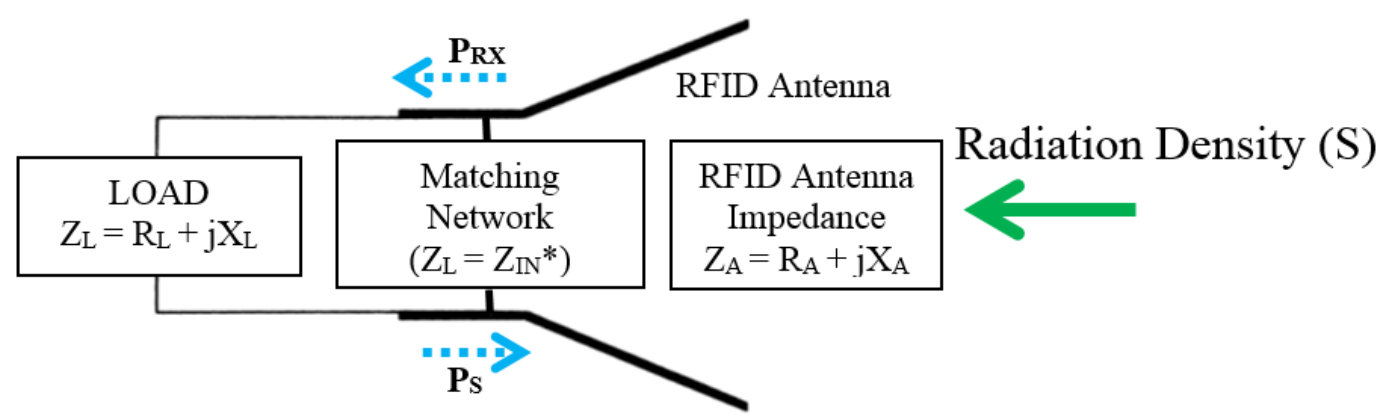

Figure 2.6. Conjugate matching achieved through matching network.

\subsection{Backscattering Modulation}

In reference to Figure 2.5 and equation (2.4), we notice that the scattering aperture depends on the load impedance attached to the antenna. In other words, if the load impedance is altered in any way the scattered power or the magnitude of power re-radiated is directly altered as well. For example, consider two extreme cases in which the power reradiated is maximized and minimized. That is, when the load in Figure 2.4 is removed and 
the antenna terminals are shorted so that $\mathrm{R}_{\mathrm{L}}=0$, and considering the antenna is resonant $\left(\mathrm{X}_{\mathrm{L}}=-\mathrm{X}_{\mathrm{A}}\right)$, this case is referred to as a resonant-short-circuit condition [34, p. 32]. Equation (2.4) then simplifies to:

$$
A_{S}=\left.\frac{V^{2}}{S R_{A}}\right|_{R_{A}=R_{r}}
$$

and when compared to equation (2.6) for a matched condition: $A_{s}=4 A_{e}$; that is, the scattering aperture $\left(A_{s}\right)$ increases to four times the maximum effective aperture $\left(A_{e}\right)[32, p$. 123], [34, p. 33]. Conversely, when the terminals of the antenna are left open $\left(Z_{L}=\infty\right)$ :

$$
\sigma=A_{S}=A_{e}=0
$$

where the antenna can no longer re-radiate or capture any power density [32, p. 123], [34, p. 34]. It is clear from equations (2.7) and (2.8) that by varying the load impedance $\left(\mathrm{Z}_{\mathrm{L}}\right)$, the scattering aperture can take on any desired values from zero to the four times the maximum effective aperture $\left(\mathrm{A}_{\mathrm{e}}\right)$.

By varying the load (i.e., load-modulation), data can be transmitted to the reader. Moreover, this method of communication is referred to as backscattering modulation [32, pp. 123, 143-145]. In RFID systems, load-modulation is conducted by varying a modulation impedance $\left(Z_{\bmod }\right)$ as a function of time, as depicted in the Figure 2.7 [32, pp. 143-145]. This impedance is internal to the RFID IC and is typically an impedance other than a short or open, as a short would run the risk of depriving the IC of power to function over a consecutive 0 -logic stream of bits $\left[7\right.$, p. 60]. Hence, $\mathrm{Z}_{\bmod }$ may be a resistive or reactive load instead [7, pp. 210-220]. Finally, it is important to note that the backscattered signal is the modulated reflected signal of the incident wave. 


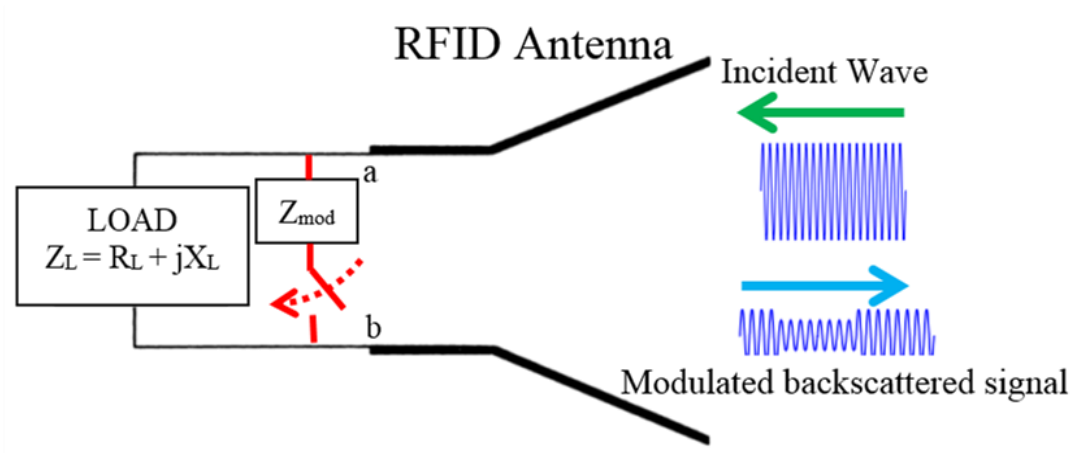

Figure 2.7. Backscatter modulation is achieved through modulating the load impedance.

As an example, the concept of backscattering modulation is analogous to a mirror or reflective surface whose reflectivity can be modulated. Accordingly, as the reflectivity is modulated, the reflected light is modulated. This concept is depicted in Figure 2.8.

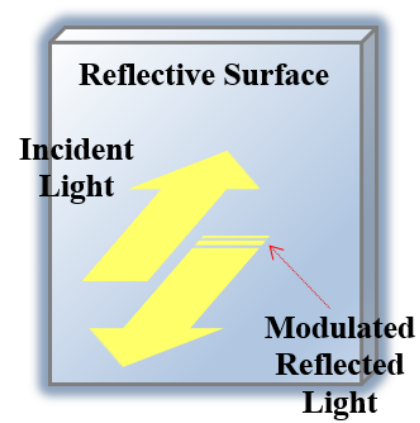

Figure 2.8. Backscatter modulation analogy using a reflective surface.

In RFID tag design, the modulation of $\mathrm{Z}_{\text {mod }}$ (from Figure 2.7) is accomplished by the RFID IC. Specifically, each RFID IC is preprogrammed with a unique Electronic Product Code (EPC) used for identification purposes [7, p. 375], [8]. The electrical load is then modulated based on this EPC [7, pp. 59-60, 366]. Moreover, the sensors in this research are designed using the Alien Higgs III RFID IC, which consists of a 96-bit EPC code [8], [35]. This RFID IC has a sensitivity (or turn-on power) of $-18 \mathrm{dBm}$ at which the IC begins to modulate the internal modulation impedance $\left(Z_{\mathrm{mod}}\right)$. Finally, at this power level, the input impedance of the RFID IC $\left(\mathrm{Z}_{\mathrm{IC}}\right)$ is $18-164 \mathrm{j}$ which remains consistent over 
the UHF RFID band of 902-928MHz [3], [36], [37]. A typical RFID setup is depicted in Figure 2.9. An RFID reader (in this research the ThingMagic Pro RFID reader was used) transmits a continuous wave $(\mathrm{CW})$ signal. The tag antenna captures this incident power density and when the turn-on power of the RFID IC is reached, the RFID IC beings to modulate the $\mathrm{Z}_{\text {mod }}$ based on the its unique identification EPC. As a result, the reflected signal is modulated. This modulated signal then returns to the RFID reader where the reader utilizes a circulator or directional coupler (assuming a monostatic antenna design) to differentiate between the transmitting and receiving signals [7, pp. 155-159]. The received signal is then demodulated in the receiver of the RFID reader.

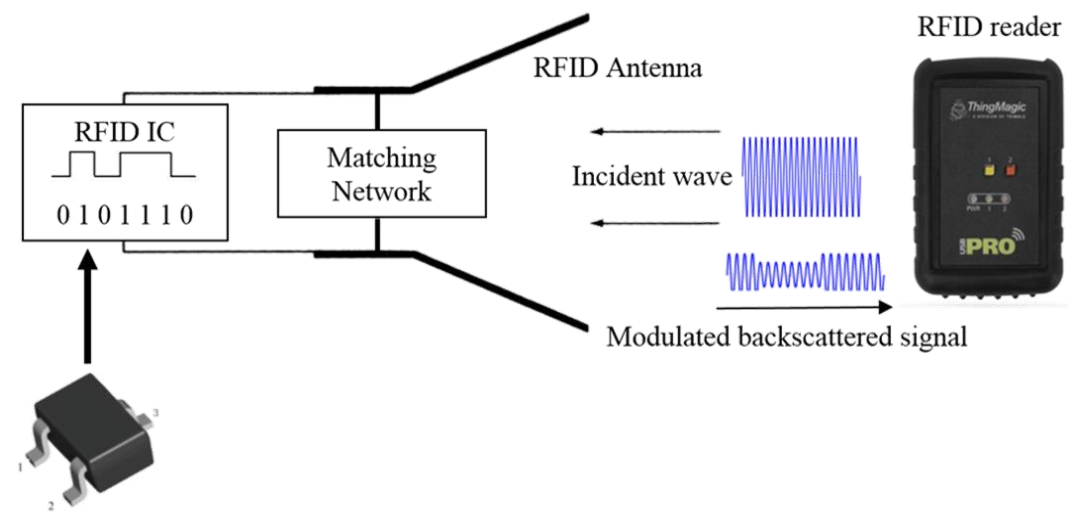

Figure 2.9. RFID reader and tag setup with modulated EPC based backscattered signal.

Furthermore, RFID readers and tags are designed to operate over the entire RFID UHF band of $902-928 \mathrm{MHz}$. The reason is to increase the probability of reading a tag. Consider a simple case with one incident path and one reflected path from the RFID reader to the RFID tag, refer to Figure 2.10. There is a possibility that the vector sum of the incident and reflected voltage-signals at the tag add destructively to inhibit tag activation [6, pp. 141-142]. This phenomenon is referred to as fading and in a real-world setting, multiple reflections can cause fading at the tag. To mitigate this issue of fading, one can 
move the position of the reader and/or the RFID tag, which will adjust the phasor-addition of the two signals and improve the total signal strength at the tag. However, instead, a simple and equal alternative is to shift the operating frequency. As a result, in the simplified case of Figure 2.10, the phase change $(\Delta \phi)$ in the total voltage signal received at the tag can be shifted by [7, pp. 93-99]:

$$
\Delta \phi=\frac{2 \pi}{c}\left(D_{2}-D_{1}\right)\left(f_{2}-f_{1}\right)
$$

where the distances, $D_{2}$ and $D_{1}$, are in meters and the frequencies, $f_{2}$ and $f_{1}$, are in Hertz.

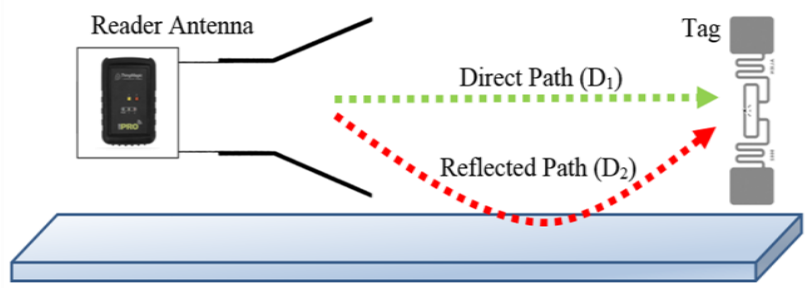

Figure 2.10. Simplified diagram of the incident and reflected wave that may cause fading.

\subsection{Friis Transmission Equation and the Power Transmission Coefficient (PTC)}

The Friis transmission equation provides the power received $\left(P_{r}\right)$ by an antenna placed at a distance, $d$, (in the far-field) from a transmitting antenna with transmit power $P_{t}$ and gain $G[33$, p. 95$]$ as follows:

$$
P_{r}=\left(P_{t} G_{t} A_{e}\right) /\left(4 \pi d^{2}\right)
$$

where $A_{e}$ is the effective aperture of the receiving antenna. As an example, a schematic of an RFID antenna terminated by an RFID IC in the receiving mode is depicted in Figure 2.11 


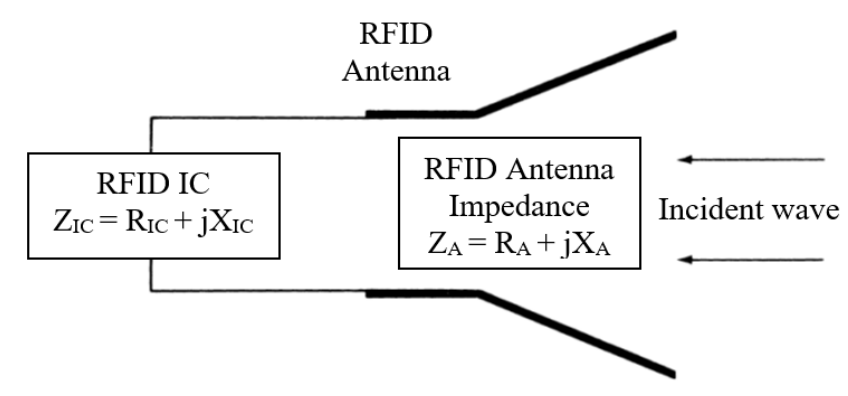

Figure 2.11. RFID antenna terminated with RFID IC impedance.

where the complex impedance of the antenna and the RFID IC are:

$$
\begin{aligned}
& \mathrm{Z}_{\mathrm{A}}=\mathrm{R}_{\mathrm{A}}+\mathrm{j} \mathrm{X}_{\mathrm{A}} \\
& \mathrm{Z}_{\mathrm{IC}}=\mathrm{R}_{\mathrm{IC}}+\mathrm{j} \mathrm{X}_{\mathrm{IC}}
\end{aligned}
$$

In addition, the power wave reflection experienced by the antenna due to the mismatch of the RFID IC complex load is provided by the following formula [38]-[39]:

$$
\Gamma=\frac{\mathrm{Z}_{\mathrm{A}}-\mathrm{Z}_{\mathrm{IC}}^{*}}{\mathrm{Z}_{\mathrm{A}}+\mathrm{Z}_{\mathrm{IC}}}
$$

Conversely, the transmitted power to the RFID IC load or the Power Transmission Coefficient (PTC) is:

$$
\tau=1-|\Gamma|^{2}
$$

By incorporating Eqns. (2.10), (2.11), and (2.12) into Eqn. (2.13), the following equation for the PTC is derived as follows [7, p. 310], [13]:

$$
\tau=\frac{4 \mathrm{R}_{\mathrm{A}} \mathrm{R}_{\mathrm{IC}}}{\left|\mathrm{Z}_{\mathrm{A}}+\mathrm{Z}_{\mathrm{IC}}\right|^{2}}
$$

Equation (2.14) is significant because it conveys the degree of matching between the RFID antenna and the RFID IC, where $0 \leq \tau \leq 1$. Moreover, during conjugate matching $\left(Z_{A}=Z_{I C}^{*}\right)$, maximum power is delivered to the RFID IC and $\tau=1[40$, p. 78], [53]. It is 
desirable to design the RFID antenna with a matching network to achieve a transmission power coefficient close to 1 . Consequently, $\tau$, as given by (2.14), is used as a figure of merit to gauge the performance of the impedance matching networks that are designed in this research. Finally, using Friis's equation from (2.9) and including the PTC from (2.14), the following formula is derived, which conveys the power received by the RFID IC from a transmitting antenna in the far-field:

$$
\mathrm{P}_{\text {chip }}=\left(\mathrm{P}_{\mathrm{t}} \mathrm{G}_{\mathrm{t}} \mathrm{A}_{\mathrm{e}} \tau\right) /\left(4 \pi \mathrm{d}^{2}\right)
$$

\subsection{Matching Network Topologies for RFID Antenna Matching}

As discussed in Section 2.4 and depicted by Figure 2.6, maximum power is delivered to the RFID IC when it is conjugately matched to the antenna. Hence, the most important aspect of designing RFID tags is the proper impedance matching. All RFID ICs present a dominantly capacitive input impedance [37]. Consequently, the T-match and inductively coupled loop matching networks are common topologies used in RFID technology to achieve the required conjugate impedance matching for maximum power transfer between the antenna and the IC [41], [42, pp. 69-71]. These matching network topologies are shown in Figure 2.12. 

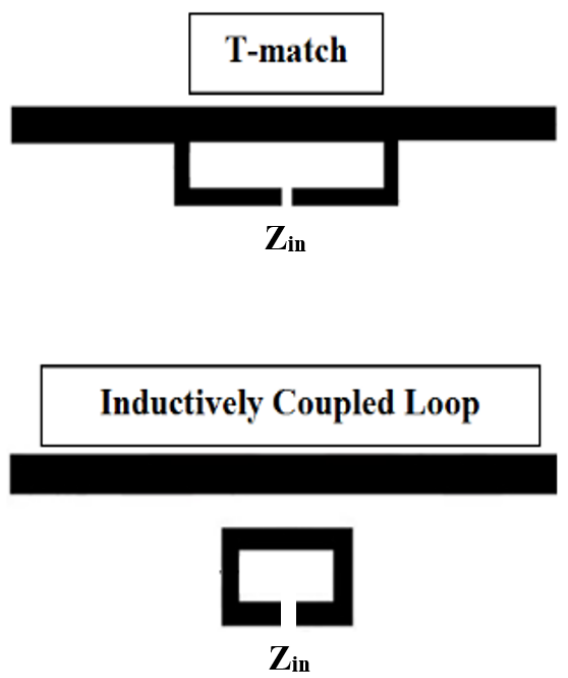

Figure 2.12. T-match and inductively coupled loop matching network topologies.

The T-match feeding network is a topology similar to the folded dipole matching network [7, pp. 327-330], [33, pp. 515-517, 531-533], [41], [42, pp. 69-71]. In the folded dipole matching network, the ends of a dipole antenna, which has a length, $l$, and wire radius, $a$, are folded and connected to form a rectangle where the width, $x$, is very small $(x \ll \lambda)$, this concept is depicted in Figure 2.13.

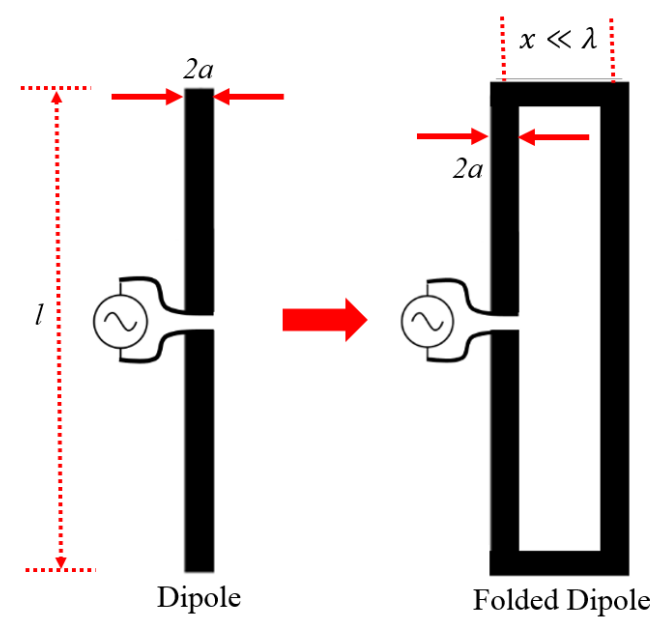

Figure 2.13. Formation of a folded dipole antenna.

The currents in any practical electrical system may be decomposed into the summation of auxiliary currents, which consist of currents that travel in the same direction (or common 
mode currents) and currents that travel in the opposite direction (or differential mode currents) [43, pp. 347-349]. Accordingly, the proper study of the folded dipole antenna then consists of the superposition of common mode currents and the differential mode currents. As a result, the input impedance of the folded dipole antenna is [33, p. 517]:

$$
\mathrm{Z}_{\text {in }}=\frac{\mathrm{V}}{\mathrm{I}_{\mathrm{in}}}=\frac{4 \mathrm{Z}_{\mathrm{TL}} \mathrm{Z}_{\mathrm{A}}}{2 \mathrm{Z}_{\mathrm{A}}+\mathrm{Z}_{\mathrm{TL}}}
$$

where $\mathrm{Z}_{\mathrm{TL}}$ and $\mathrm{Z}_{\mathrm{A}}$ represents a shorted transmission line input-impedance whose length is half the antenna-length and the antenna input impedance of an ordinary dipole antenna without any matching, respectively. The response of the folded dipole matching technique is best understood when viewed as a transformer model as shown in Figure 2.14.

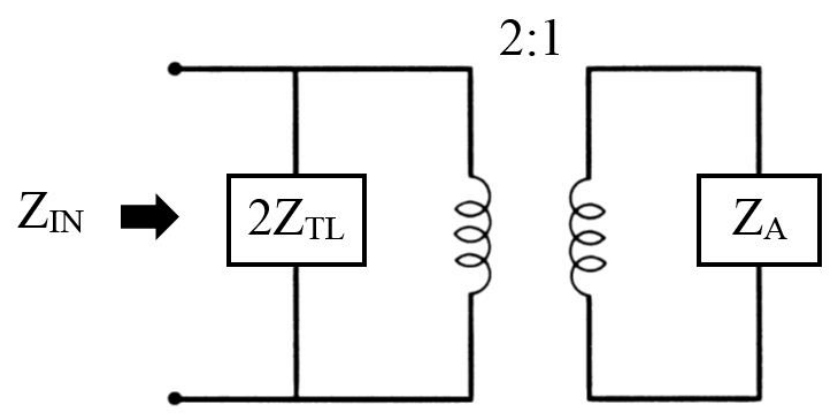

Figure 2.14. Transformer model of the folded dipole antenna.

Furthermore, by adjusting the geometry or dimensions of the inner arm of the folded dipole antenna (as shown in Figure 2.15); namely, the radius, $a^{\prime}$, length, $l^{\prime}$, and width, $x$, additional freedom in the matching at the input port is obtained. 


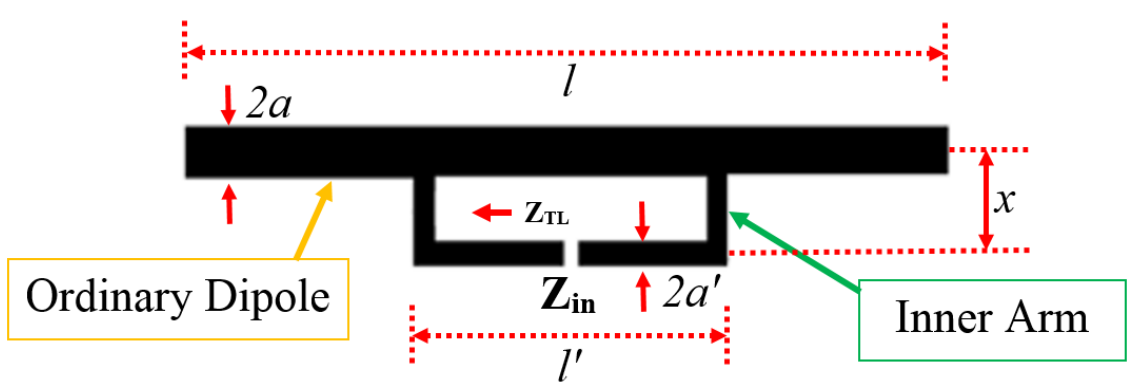

Figure 2.15. Customizable input impedance due to the modified inner arm geometry.

Specifically, due to this new geometry of the inner arm, the common-mode current division is no longer unity, since the antenna is no longer symmetric along the length axis. In other words, as a result of this asymmetry, the total current at the input port is not divided equally between the inner arm and the antenna dipole. To account for this non-uniform current distribution, the current division factor, $\alpha$, is introduced and is based on the geometry of the inner arm as follows:

$$
\alpha=\frac{\ln \left(\frac{\mathrm{x}}{\mathrm{a}^{\prime}}\right)}{\ln \left(\frac{\mathrm{x}}{\mathrm{a}^{\prime}}\right)-\ln \left(\frac{\mathrm{a}}{\mathrm{a}^{\prime}}\right)}
$$

where this formula assumes cylindrical conductors. Moreover, the function of the geometry based current division factor, $\alpha$, in customizing a specific input impedance at the port is effectively depicted in the transformer model as conveyed in Figure 2.16. 


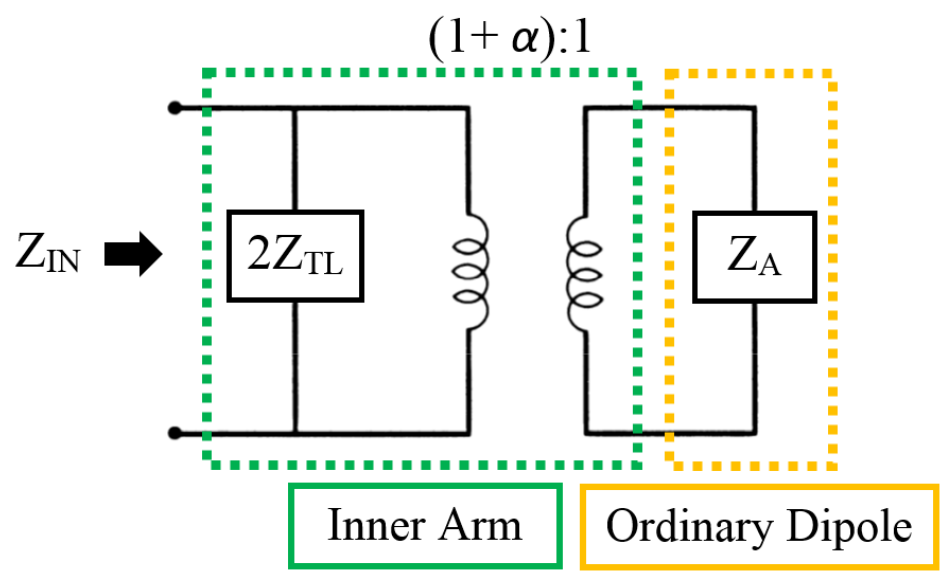

Figure 2.16. Transformer model of the T-matching network.

Finally, from Figure 2.16, the input impedance using the T-matching network is as follows:

$$
\mathrm{Z}_{\mathrm{IN}}=\frac{2 \mathrm{Z}_{\mathrm{TL}}\left[(1+\alpha)^{2} \mathrm{Z}_{\mathrm{A}}\right]}{2 \mathrm{Z}_{\mathrm{TL}}+(1+\alpha)^{2} \mathrm{Z}_{\mathrm{A}}}
$$

where, as in the case of the folded dipole antenna, $\mathrm{Z}_{\mathrm{A}}$ is the input impedance of an ordinary dipole antenna without any matching network and $\mathrm{Z}_{\mathrm{TL}}$ is the shorted transmission line input impedance of half the length of $l^{\prime}$, refer to Figure 2.15 [33, pp. 531-533], [44].

Furthermore, a dipole antenna can be modelled as a series circuit comprising of a resistance $\left(\mathrm{R}_{\mathrm{ANT}}\right)$, an inductance ( $\left.\mathrm{L}_{\mathrm{ANT}}\right)$, and a capacitor $\left(\mathrm{C}_{\mathrm{ANT}}\right)$ [7, p. 314-315]. Therefore, the antenna can be mutually coupled to an inductive feed-loop through the antenna inductance $\left(\mathrm{L}_{\mathrm{ANT}}\right)$. This is the mechanism through which the inductively-coupled-loop matching network operates. This topology, as shown in Figure 2.17, adds the additional inductive reactance needed to achieve a conjugate impedance match [41]. The concept is to attach the RFID chip to the inductive-feed-loop with the proper dimensions $(a, b$, and $w$ ), and adjust the separation, $d$, accordingly to acquire the proper strength of mutual inductance, $M$, and achieve the desired conjugate matching at the feed port [6, pp. 83-84]. 


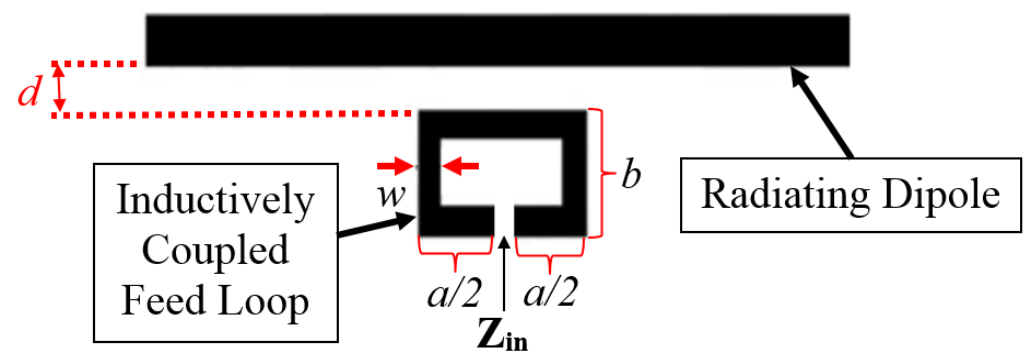

Figure 2.17. The input impedance is customizable by adjusting the dimensions $a, b, w$, and $d$.

As in case of the folded dipole and T-matching networks, the functionality of this matching network is best understood when viewed as a transformer model as conveyed in Figure 2.18 .

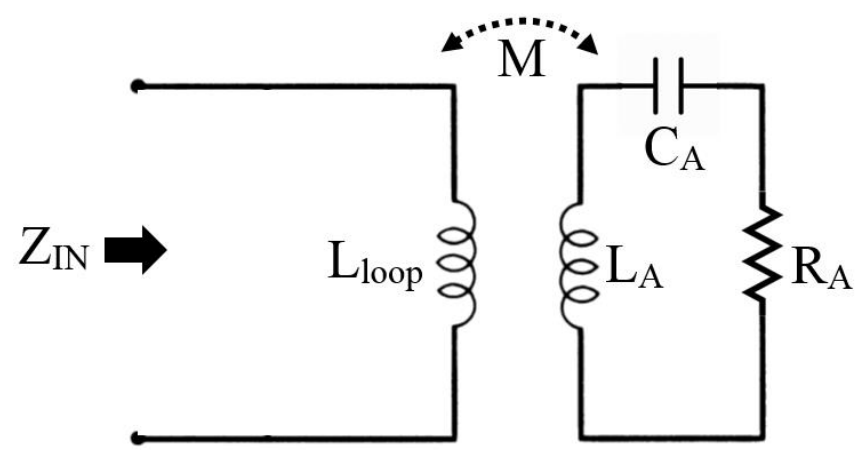

Figure 2.18. Transformer model for the inductively coupled loop matching network. Accordingly, the input impedance $\left(\mathrm{Z}_{\text {in }}\right)$ is [45]:

$$
\mathrm{Z}_{\mathrm{IN}}=\mathrm{Z}_{\text {loop }}+\frac{(2 \pi \mathrm{fM})^{2}}{\mathrm{Z}_{\mathrm{A}}}
$$

where $Z_{\text {loop }}=j 2 \pi f L_{\text {loop }}, Z_{A}=R_{A}+j 2 \pi f\left(L_{A}+C_{A}\right)$, and $M$ is the mutual inductance, which can be derived analytically. 


\section{CHAPTER 3}

\section{RELATED WORK}

Commercial RFID sensors for monitoring the temperature of perishable goods within the Cold-Supply-Chain (CSC) are currently available. Additionally, a variety of novel RFID temperature sensor designs have been published and they are applicable to the CSC. Both these categories of sensors are examined in the following sections.

\subsection{Commercially Available Temperature Sensors for the CSC}

Currently, commercially available RFID temperature sensors are available for the CSC [28]. For example, the Sensor Temperature Dogbone from Smartrac [46]. This RFID sensor-tag consists of a passive design that utilizes the Axzon Magnus S3 IC, which serves as the temperature sensor. The analog temperature data is digitized and wirelessly downlinked to the reader through a specialized EPC. Unfortunately, the digitized data requires additional specialized software for decoding and processing the temperature data. This adds an additional layer of complexity, which makes the CSC dependent and vulnerable to issues related to malfunctioning software and/or corresponding hardware.

As another example, there are the Freshtime Tags from Infratab [47], [48]. These sensors monitor and track perishable items in the CSC. Moreover, each sensor consists of a separate battery-powered temperature sensor separated by a certain length from a writable-RFID IC from which the sensor data is wirelessly extracted using the RFID platform. Also, these tags are made of various lengths ranging from $4 \mathrm{~cm}$ to few hundreds of centimeters long to separate the RFID IC from the temperature sensor. This is done to accommodate various dimensions of packages and position the temperature sensor as close 
to the perishable item as possible with the RFID IC exposed external to package, where it can be easily read. Unfortunately, this product requires batteries that require replacement by the manufacturer approximately on a yearly basis. Moreover, specialized software is also required to process the data. Also, these tags serve as data-loggers where limited finite temperature samples are taken. It then takes these temperature samples to predict the shelflife of a product [28]. Finally, wherever necessary, the physically lengthy design of various tags may be cumbersome during installation and not feasible for fast-pace processing scenarios, such as the CSC.

Finally, the IDS-SL13A from IDS Microchip AG is a semi-passive RFID temperature data-logger [49]. The IDS-SL13A is a single-chip smart device that can be integrated with other types of sensors, such as, accelerometers, motion detectors, pressure sensor, etc. In addition to the on-board analog temperature sensor, all data from the additional sensors is sampled, digitized, and stored on an on-board memory bank. Finally, all data can be retrieved wirelessly through an RFID communication protocol. Furthermore, this device is advantageous due its small form-factor. Unfortunately, this temperature sensor utilizes a power source, limiting the life-span to approximately one year. Also, a customized software is required to decode the data as well.

Finally, there is the I-Q series of active RFID temperature sensors from Identec Solutions [50]. These series of RFID temperature sensors provide data-logging of temperature data, which can be retrieved wirelessly up to $100 \mathrm{~m}$ away. However, these devices utilize batteries and specialized software. Also, due to the complexity of the active design, these sensors have a high cost, which makes their use impractical; especially when a high quantity of these sensors must be used. 
In summary, although these sensors present satisfactory methods of monitoring temperature conditions in the CSC, they have limitations in one way or another. Particularly, they are costly since their semi-passive and active designs equate to additional complex circuitry. Also, they require batteries; therefore, they have a limited life-span and/or require constant battery replacements (in some cases this can only be done by the manufacturer, which further complicates the process). Furthermore, these designs require specialized software to decode the temperature data and as mentioned earlier, this creates vulnerabilities in the robustness of the CSC. Finally, many of these sensors serve as dataloggers. This means that sensing data is taken at various time-intervals, which creates the possibility to miss events, where adverse temperature conditions may exist. This limiting capability is dangerous as bacteria growth can double in just twenty minutes in excessive temperatures (i.e., near $70^{\circ}-120^{\circ} \mathrm{F}$ ) [51].

\subsection{Published Temperature Sensors}

The increased interest in wireless communication and identification has led to the development and expansion of numerous novel sensor technologies. The RFID platform operates efficiently in highly-dense and cluttered environments and consists of a simplistic and cost-effective network of tags and readers. As a result, RFID technology is often examined for sensing applications by researchers. The following are unique temperature sensors that are applicable to the Cold-Supply-Chain.

The first sensor is a passive RFID tag for heat sensing applications, which is developed by researchers at Tampere University of Technology in Finland [52]. This sensor consists of a customized designed antenna that is placed on a substrate (or dielectric) 
of paraffin wax. As the paraffin wax begins to melt due to high temperature exposure, the properties (of the surrounding medium) are altered, which de-tunes the operating frequency of the tag. This de-tuning in the operating frequency conveys the change in the surrounding temperature.

Additionally, another sensor developed by researchers at Massachusetts Institute of Technology and University of Kansas expresses changes in the temperature through the controlled shifts in the operating frequency within the UHF RFID frequency band [53]. The sensor consists of a stationary dipole antenna. Under this antenna, at some distance, a metal plate is positioned. Moreover, under this metal plate, a temperature-activated support made from a shape memory polymer is placed. At a certain temperature (in this case $7^{\circ} \mathrm{C}$ ), this polymer transitions from a flexible state to a rigid-state at which point it raises the metal plate closer to the stationary antenna. Through the change in the parasitic capacitance between the metal plate and the antenna, a change in the operating frequency occurs. Finally, the degree of displacement in the height of the metal plate is considered in the design to develop controlled shifts in the operating frequency.

Thirdly, a temperature sensor is designed which conveys changes in temperature by switching operation between one of two onboard RFID ICs [54]. This sensor comprises of a customized dipole antenna structure which consists of a dual T-match feed networks to accommodate both RFID ICs [25]. Moreover, a temperature switch is made from a shape memory alloy (i.e., Nitinol which is a Nickle-Titanium alloy). The switch is situated in such a way that it shorts the feed connected to one of the RFID ICs. At elevated temperatures, the Nitinol switch is triggered into a rigid state at which point the short is 
eliminated and the second RFID IC is now activated. When two IDs are retrieved by the reader, a temperature threshold has been crossed.

Finally, another temperature whose operating frequency is dependent on the ambient temperature is designed by researchers at Tampere University of Technology in Finland and the University of Mississippi [8]. The sensor consists of a dipole antenna that is matched to an RFID IC using a combination of meandering transmission lines and a Tmatching network. A water-pocket is placed above this matching network. Accordingly, as the temperature changes, the relative permittivity of the water is altered as well. This change in the relative permittivity affects the stray capacitance emitted from the meandered lines. The functionality of this sensor depends on the inverse-linear relationship of temperature and the relative-permittivity of water. That is, as the temperature increases, the relative permittivity decreases, the stray capacitance then decreases, and finally the resonant frequency increases. In summary, as temperature increases, the resonant frequency increases. Unfortunately, this design is limited to the freezing and boiling temperatures of water.

All the designs mentioned in this section are cost-effective and can be uses in the CSC. However, the first three are limited because they can be used only one-time or they need resetting after a single use (this is a concern for sensors that are installed in locations that are physically difficult to access). Finally, the last sensor presented is limited to detecting freezing and boiling temperatures of water. Hence, these are not convenient solutions. A pragmatic design would be reusable and be able to detect multiple temperature thresholds without any form of resetting. Temperature sensors with these improved features are designed and presented in this research. 


\subsection{Summary of Available Temperature Sensing Technologies in the CSC}

The various temperature-sensor technologies described in this chapter, can be divided into three categories. That is, active, semi-passive, and passive RFID designs. The advantages and disadvantages of semi-passive and passive tags are compared in Table 3.1. Moreover, the active sensor design utilizes a completely different radio design topology (i.e., it does not communicate using backscatter modulation) and it is beyond the scope of this research; therefore, it has not been included in the table.

Table 3.1. Summary of advantages and disadvantages of the available RFID temperature sensor technologies.

\begin{tabular}{|c|c|}
\hline Semi-passive & Passive \\
\hline Require batteries (short term) $X$ & No batteries (long term) \\
\hline $\begin{array}{c}\text { Data logging (do not operate } \\
\text { continuously) } \mathbf{X}\end{array}$ & $\begin{array}{c}\text { Single use or require resetting (do not operate } \\
\text { continuously) } \mathbf{X}\end{array}$ \\
\hline Costly design $\mathbf{X}$ & Cost effective design $\checkmark$ \\
\hline Improved range $\checkmark$ & Reduced range $\mathbf{X}$ \\
\hline Require specialized software $X$ & $\begin{array}{c}\text { Do not require specialized software (except the } \\
\text { Sensor Temperature Dogbone) }\end{array}$ \\
\hline
\end{tabular}

Finally, the disadvantages of the current technologies provide the functional guidelines as detailed in Section 1.2 for the novel sensors developed by this research. In summary, the sensors that are developed here operate continuously, autonomously, and are battery-free. 


\section{CHAPTER 4}

\section{LIQUID CRYSTAL ELASTOMERS (LCEs)}

\subsection{Liquid Crystals, Polymers, and Elastomers}

A compound in the liquid state is isotropic when the properties (e.g., viscosity and surface tension) are uniform in all directions. In contrast, crystals exhibit properties which vary with direction; consequently, they are anisotropic. Liquid-Crystals (LC) consist of compounds that exist in a phase that demonstrate properties of both liquid and crystalsolids; that is, fluidity and order in molecular-orientation, respectively [55], [56]. Furthermore, polymers are large molecules formed by the continuous bonding of smaller molecules, known as monomers [57]. The polymerization (or combining) of monomers that exhibit liquid crystal properties couple the molecular-orientation order of the molecules into an elastic material. This provides the potential of such polymers to be customizable and offer reversible shape changing in desired directions when stimulated [56]. However, this reversible actuation (return to the natural state without any physical intervention) is actually achieved through cross-linking these polymer chains in an aligned (ordered) state as shown in Figure 4.1 [58], [60]. 


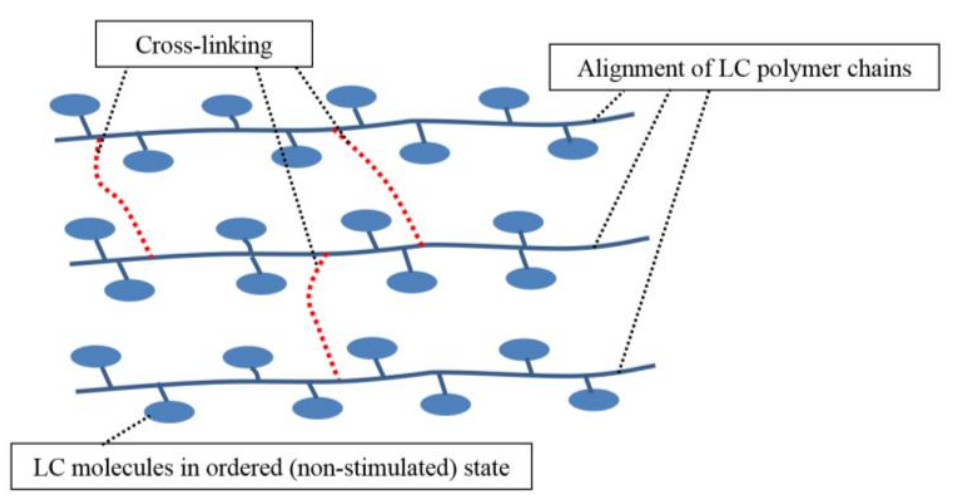

Figure 4.1. Cross-linked LC polymer chains aligned in the ordered state.

Furthermore, the liquid crystals in the polymer chain exist in the nematic phase. That is, they maintain orientational order. Upon applying a heat stimulus, the molecules undergo a phase transition with a large disruption in the molecular order into an isotropic state [59]. Consequently, this phase transition results in the actuation of the substrate, which is made from these aligned polymer chains. Moreover, due to the aligned polymer cross-linking, the substrate returns to the original shape as the stimulus is removed. The reversible actuation through the transition of the nematic and isotropic states are depicted in Figure 4.2.

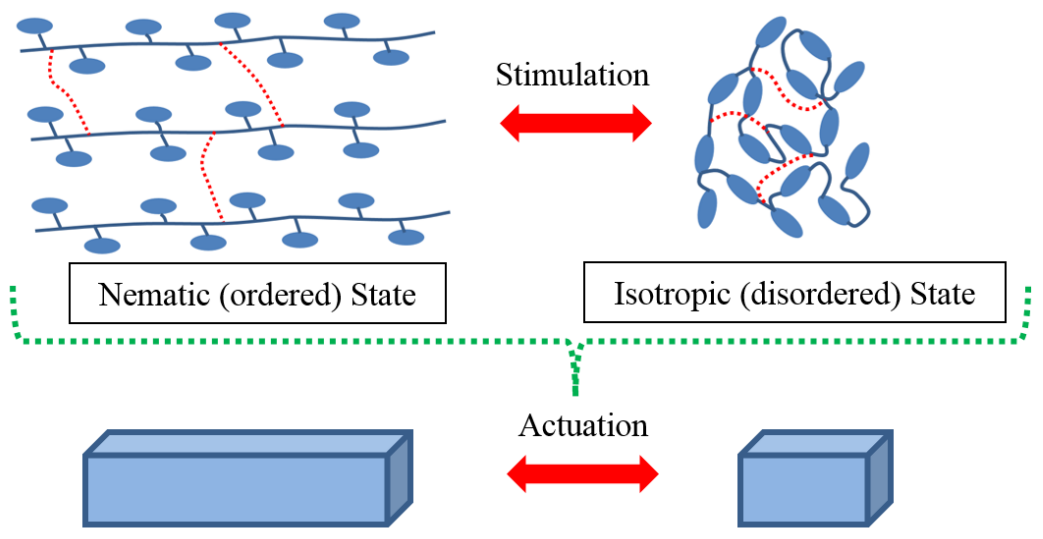

Figure 4.2. Actuation due to the LC polymer transition from the nematic phase to the isotropic phase when stimulated.

The general liquid-crystal-polymers described above differ from liquid-crystalelastomers (LCEs) by the extent to which molecular-orientation-order can be diminished 
in the material when presented with a stimulus, such as, temperature-differential, UV light, and various solvents. Additionally, this disruption in the molecular-orientation-order is a quantifiable metric [56], [60]. Accordingly, in the case of LCEs, a large differential in the orientational order takes place when excited with a stimulus [56]. In this research, we focus on temperature reactive LCEs or thermotropic LCEs. In summary, LCEs are temperatureresponsive polymers that undergo large, reversible, anisotropic shape changes without any external load application [58], [59], [60].

\subsection{LCE Thin Film Actuation}

The orientation of the liquid crystal molecules in the polymer chain define the nematic director. When a heat-stimulus is applied, the aligned LCE polymer chains contract along the nematic director and a net strain is experienced in the perpendicular directions, and as the stimulus is removed the polymer chains revert back to their original configurations [62], [60]. Various methods to align and arrange LCE polymer chains exist with high spatial resolution to create thin film LCEs. However, these thin films are limited to a maximum thickness of $100 \mu \mathrm{m}$ [60]. Moreover, through a graded variation in the orientation of the nematic directors in respect to the thickness of LCE films, one can obtain customized twists and turns from LCE films when they are stimulated, such a graded nematic director variation is shown in Figure 4.3. Thus far, this LCE technology has proven to be resilient. According to [61], 100,000 actuation cycles have been successfully conducted without any failure. 


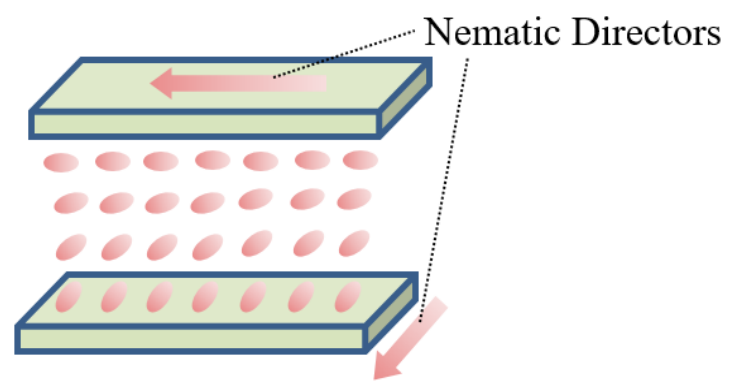

Figure 4.3. Hierarchical neamatic vector to obtain various twist and curves.

Novel reversible temperature-responsive helical antennas using thin-film LCEs with graded nametic directors were designed, fabricated and tested in [59]. A thin aluminum layer was glued to the LCE to create the conducting antenna traces. As the temperature increased (from $30^{\circ} \mathrm{C}$ to $92^{\circ} \mathrm{C}$ ), the antenna design transformed from a linear element to a helical element, which varied the operating frequency and radiation pattern.

\subsection{Printed LCE Technology}

4D printed technology refers to $3 \mathrm{D}$ printed structures that can transform their shape after printing [52], [60]. As mentioned earlier, LCE films are limited to a thickness of $100 \mu \mathrm{m}$. Therefore, they are restricted to the amount of actuating force that they can exert [62]. Fortunately, the additive manufacturing process conveyed by 3D printing provides a constructive solution to this limitation of thin film LCEs. That is, it allows for the additional layering of the LCE filament, which provides a larger exertion force. Also, the shearingforce inherent to the $3 \mathrm{D}$ printing motion can be used to induce the desired alignment of the LCE filament. In other words, the molecular alignment of the LCE filaments coincide with the print path [52], [60], refer to Figure 4.4. 


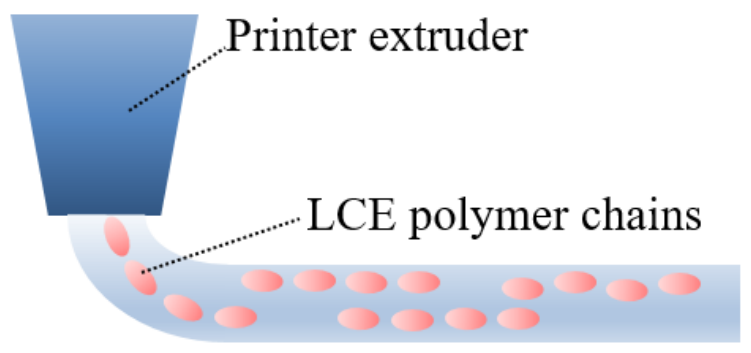

Figure 4.4. Molecular alignment of the LCE polymer chains coincides with the print path.

Therefore, depending on the print path, one can pre-program the desired anisotropic shape-response for when a stimulus is applied [52], [60]. Furthermore, to obtain additional design variability for 4D printed LCE actuators, graded or hierarchical control along with the $3 \mathrm{D}$ printing shearing method can be utilized and this was first demonstrated in [60]. Accordingly, innovative actuator designs have been created. The primary example is the Archimedean spiral, which transforms into a dome shape upon stimulation. This is possible because the nematic director varies azimuthally around the center point [52], [60]. As another example, the bilayer-thick-twisting-film that consists of two layers in which the molecular orientation is varied through the thickness of each layer. The orientation difference between the top and bottom layers create various twisting orientations [60]. Moreover, there is the thick-checkered-porous structure, which consists of 16 layers of LCE film. Each layer consists of a $90^{\circ}$ molecular-phase orientation difference. Hence, upon receiving a stimulation, the layers contract and the pores shrink in area [60]. Finally, all these structures are stimulated with increasing temperature that ranges from $30^{\circ} \mathrm{C}$ to $160^{\circ} \mathrm{C}$.

In this research, we have utilized the 4D printed Archimedean spiral design. This design is chosen because it transforms into a cone shape (when stimulated), which is a 
practical design for lifting antennas and actuating switches. As indicated earlier, this design is fabricated by directing the print-path of the LCE filament in an Archimedean chord pattern. The result is an Archimedean spiral design that consists of a radial nematic director that varies azimuthally about the center of the design (around a single point), where a defect exists, refer to Figure 4.5. A defect is a point or region, where the orientational order of the molecules drops [63]. Additionally, the alignment of the LCE filaments represent a vector field of the liquid crystal molecule orientations and the defect in the LCE actuator can be characterized by the number of times the vector field of the liquid crystals alignments wind around a loop encircling the defect in a single anticlockwise rotation [63]. Hence, for the Archimedean spiral design, the director pattern is observed to have a +1 topological defect, this is depicted in Figure 4.5 [13], [53], [60].

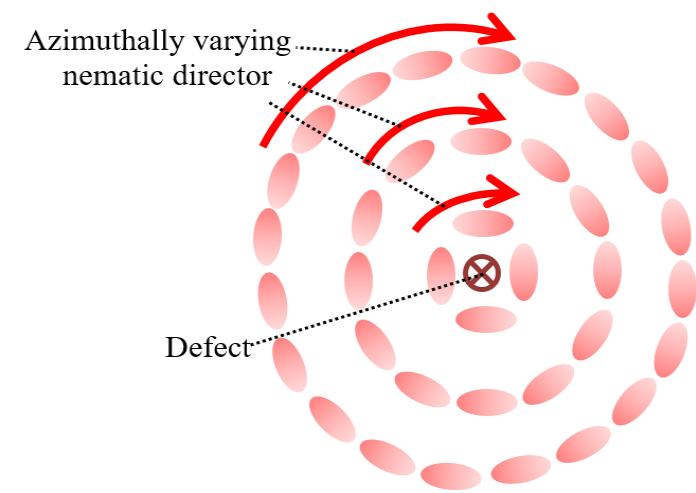

Figure 4.5. Azimuthally varying nematic vector of the Archimedean spiral design. When a heat-stimulus is applied (approximately $160^{\circ} \mathrm{C}$ ) the liquid crystals undergo a phase transition and the LCE filaments (or polymer chain) contract azimuthally (along their direction of alignment). Consequently, the center point of the design is actuated in the direction normal to the plane in which the actuator is placed, the overall shape is a cone cell as depicted by the fabricated Archimedean design in Figure 4.6. 


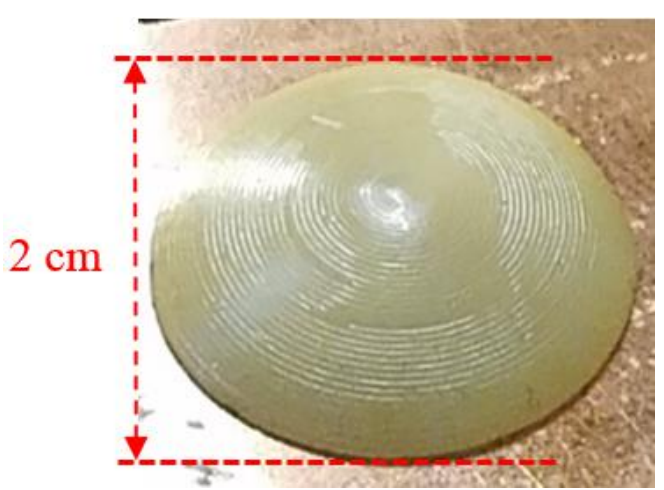

Figure 4.6. Fabricated 4D printed Archimedean spiral design in the stimulated state.

In the context of this research, LCE actuators are integrated with radiating antenna elements for the controlled temperature-dependent movement translating to a measurable temperature-dependent variation in the antenna performance. Consequently, the required approximate lifting capacity from the LCE actuator ranges from 15 to 25 gf (gram-force). Conversely, the favorable application of LCE actuators are for optics, medicine, microfluidics, and robotics [62]. Naturally, these applications are associated with movement that require small forces. Therefore, the inherent lifting capacity of a single 4D printed LCE actuator is insufficient for the application in this research. To resolve this issue, individual 4D printed LCE actuators (or cells) have been combined in an array formation. This array design may be customized based on the position and number of actuator cells to provide the required lifting capability [13]. The 4D printed LCE array, which is used in one of the sensors developed by this research, uses heat-reactive LCE Archimedean spiral cells and it is depicted in Figure 4.7. 


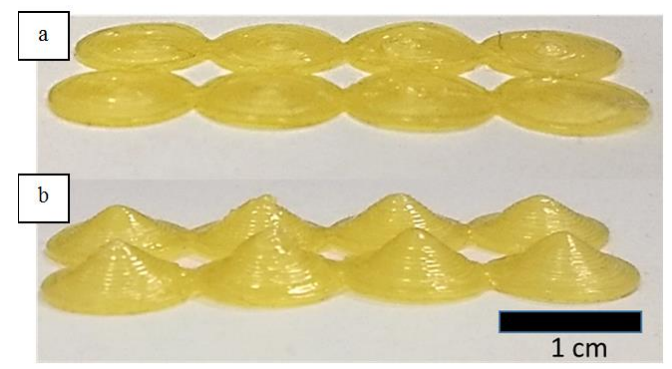

Figure 4.7. Heat reactive 4D printed LCE array: (a) relaxed state (b) excited state.

\subsection{Cold Reactive Liquid Crystal Elastomers}

Thus far, we have discussed the theory of innovative heat-reactive 4D printed LCE actuators. In addition to that, for the first time, 4D printed cold-reactive LCE actuators have been designed and fabricated by our collaborators, the Ware Research Group in the University of Texas at Dallas [64]. These state-of-the art cold-reactive actuators have been incorporated into two novel passive RFID temperature sensors developed by this research.

Notably, no chemical difference between heat- and cold-reactive 4D printed LCE actuators exists. However, their difference is in the actuation mechanism and direction of strain in the liquid crystal elastomer chains. That is, at lower-temperatures (approximately $-9^{\circ} \mathrm{C}$ to $-12^{\circ} \mathrm{C}$ ), the molecules undergo a thermal-expansion as opposed to a phasetransition for high-temperature reactive LCE actuators. Therefore, the thermal-expansion causes elongation of the radially aligned polymer chains. As a result, strain (deformation) occurs in the direction perpendicular to the alignment. In other words, the shape change encountered in cold-reactive LCE actuators is opposite to that of heat-reactive LCE actuators. Consequently, circular cells with radially-aligned LCE polymer chains are designed to give the same dome-shaped response as the heat reactive Archimedean spiral designs (refer to Figures 4.5 and 4.6). A diagram of a single cold-reactive LCE cell actuator 
showing the direction of actuation of the polymer-alignments and resulting strain is depicted in Figure 4.8. Also, the corresponding fabricated design is shown in the figure.

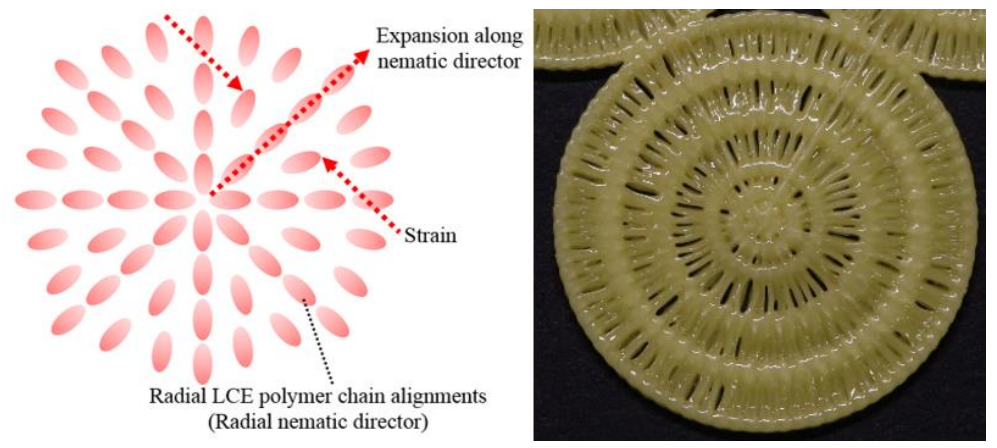

Figure 4.8. Cold-reactive LCE cell with radial alignment.

Finally, to improve the lifting capacity of this LCE, an array which consists of three coldreactive 4D printed circular cells with radially-aligned LCE polymer chains is fabricated and incorporated in two of the RFID passive temperature sensors developed by this research (see Figure 4.9). Also, the array in its relaxed and excited states is shown in Figure 4.9 .
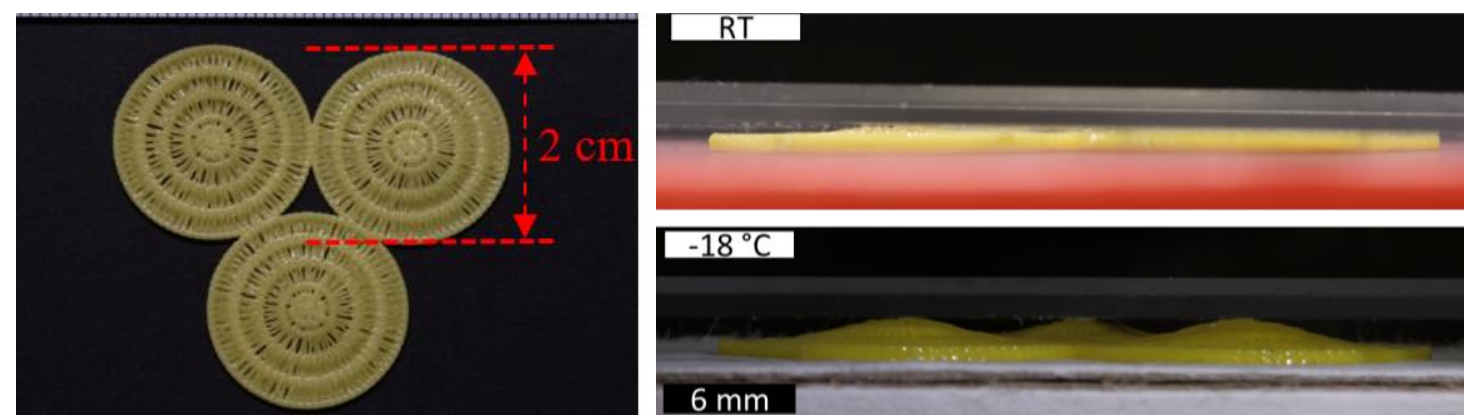

Figure 4.9. Array of cold-reactive LCE cells (left). Array in the relaxed (right upper) and excited states (right lower).

Furthermore, it is important to note that due to the difference in the actuation mechanisms (i.e., thermal expansion for the cold-reactive LCEs and phase-transition for the heat-reactive LCEs), the magnitude of the shape deformation is reduced for coldreactive LCEs. That is, for our cold-reactive 4D printed radial array, a displacement of approximately $1.23 \mathrm{~mm}$ (at approximately $-9^{\circ} \mathrm{C}$ to $-12^{\circ} \mathrm{C}$ ) is achieved. This array design 
provides a lifting capacity of $15 \mathrm{gf}$, which was sufficient for the sensors designed in this research. Conversely, the heat-reactive 4D printed Archimedean spiral array has a displacement of $4 \mathrm{~mm}$ and a lifting capacity of $25 \mathrm{gf}$ (at approximately $160^{\circ} \mathrm{C}$ ). This actuation displacement comparison is shown in Figure 4.10.

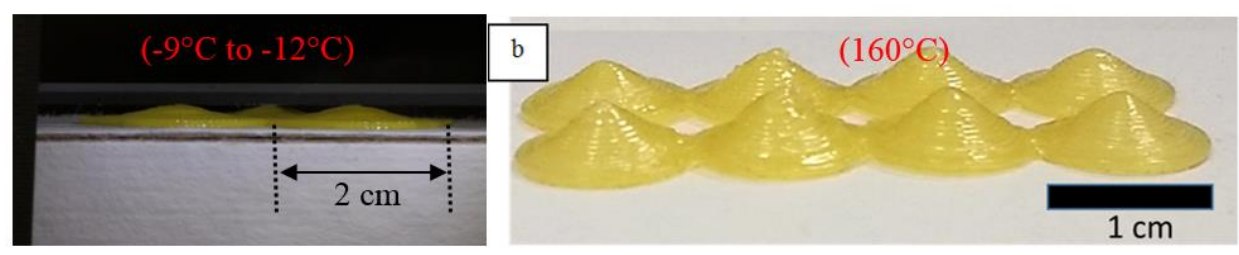

Figure 4.10. Actuation displacement comparison. (left) $1.23 \mathrm{~mm}$ for cold-reactive LCE and (right) $4 \mathrm{~mm}$ for hot-reactive LCE.

\subsection{Summary of Shape-Memory Materials}

In this research, Liquid Crystal Elastomers are utilized. They are stimuli-responsive polymers. Specifically, in this research LCEs, which respond to temperature, are used. LCEs undergo large and reversible shape deformations when they are subjected to stimuli. Also, by controlling the alignments of the polymer chains and through 4D printing techniques, LCE structures can be programmed to morph into various complex shapes [9]. Additionally, the fabrication process associated with LCEs is straightforward, costeffective and easily repeatable with the fabrication time being within one day for 4D printed LCE structures. LCEs are non-toxic and the materials required are also inexpensive and readily accessible [13], [65]. They are ideal candidates for applications requiring mechanical actuation as they operate successfully in air as opposed to being submerged in aqueous solutions or encapsulated into channels [9], [13]. Finally, other stimuli-responsive materials with programmable shape-morphing capability exist. Examples include shape memory alloys, liquid metals, hydrogels and gel polymers [54], [66]-[68]. 
Shape memory alloys are a group of metallic materials that have the ability to shift or morph their structure to a pre-defined (or programmed) shape when they are subjected to an applied heat stimulus [66]. However; in most cases, shape memory alloys cannot revert to their original shape once the stimulus is removed (i.e., no memory) [54], [66]. Thus far, Nickel-Titanium alloy (NiTi or Nitinol) has shown to be the most effective mixture in displaying programmability [66], [69]. Also, NiTi exhibits other good properties, such as, high-electrical conductivity and biocompatibility. However, manufacturing Nitinol can be complicated as Titanium becomes reactive in the alloy and melting must be done in a vacuum or in an inert atmosphere. Subsequently, standard hot-forming processes, such as, forging and extrusion must be applied to form the final product. Finally, welding and soldering of NiTi is usually a difficult task [66].

A Liquid Metal is an alloy with a very low melting point and it exists in a liquid state near room temperature. Recently, research in Gallium based liquid metals has increased. This is because these alloys flow easily and can therefore be shaped using guided channels [67], [70]. In addition, Gallium-based liquid metals have valuable properties, such as, high thermal and electrical conductivity, and they are non-toxic as opposed mercurybased alloys. Furthermore, the Eutectic Gallium Indium alloys (EGaln) are important alloys that have been studied extensively by researchers. Accordingly, when liquid metal alloys are combined with elastomeric microfluidic channels, they can create programmable shape-shifting structures that can flex, twist, and stretch when an external force is applied. Moreover, these structures have the capability to return to their original shape using an applied external force. A leading application for liquid metal structures is reconfigurable antennas [67]. Unfortunately, liquid metals are difficult to work with in the sense that they 
cannot be exposed to air, results in an oxide layer forming on the surface. This is undesirable as it will result in poor physical and electrical contact [70].

Finally, hydrogels consist of soft materials which autonomously react and change their shape due to an applied external signal [68]. Specifically, they consist of hydrophilic polymers that swell and hold water while maintaining their structure due to polymer crosslinking [71]. When subjected to an external heat stimulation a large volumetric change is realized, which can be controlled. That is, expansion and contraction is attained by absorbing or expelling the surrounding solution through an applied stimulation. These changes are reversible as the shape is restored once the stimulus is removed. Moreover, these volumetric changes are ideal for applications that utilize microfluidics and mechanical actuators. The synthesis of hydrogels is an involved process requiring specialized equipment and chemicals [68], [71]. Unfortunately, hydrogels must be submerged in aqueous solutions to operate correctly [68].

By comparing the different shape-shifting materials, we can conclude that LCEs are very well suited for developing passive temperature sensors, which is the purpose of this research. Their primary advantages are that they are programmable and reversible (memory) and they provide the largest shape deformation. Also, LCEs have an optimized, easily repeatable, and simple fabrication process. Moreover, there are no health risks involved when touching LCEs and they operate well in air. To summarize the discussion presented in this section, all the shape-morphing materials are compared based on important features in Table 4.1 below: 
Table 4.1. Comparison of Shape-Morphing Materials

\begin{tabular}{|c|c|c|c|c|}
\hline $\begin{array}{l}\text { Technology } \\
\text { Capability }\end{array}$ & $\begin{array}{l}\text { Liquid Crystal } \\
\text { Elastomers }\end{array}$ & $\begin{array}{c}\text { Shape Memory } \\
\text { Alloy (Nickel } \\
\text { Titanium) }\end{array}$ & $\begin{array}{l}\text { Liquid Metals } \\
\text { (Gallium Indium } \\
\text { Alloy) }\end{array}$ & $\begin{array}{l}\text { Hydrogels (Gel } \\
\text { Polymers) }\end{array}$ \\
\hline $\begin{array}{l}\text { Programmable } \\
\text { (Shape) }\end{array}$ & Yes & Yes & $\begin{array}{l}\text { No (require guided } \\
\text { channels) }\end{array}$ & Yes \\
\hline $\begin{array}{c}\text { Memory } \\
\text { (return to } \\
\text { original state } \\
\text { after removal of } \\
\text { stimulus) }\end{array}$ & Yes & No & $\begin{array}{c}\text { No } \\
\text { (require an external } \\
\text { force) }\end{array}$ & Yes \\
\hline $\begin{array}{l}\text { Magnitude of } \\
\text { shape } \\
\text { deformation }\end{array}$ & $\checkmark$ & & $\begin{array}{l}\text { NA (require guided } \\
\text { channels) }\end{array}$ & \\
\hline $\begin{array}{l}\text { Simplified } \\
\text { fabrication } \\
\text { process }\end{array}$ & $V$ & & & \\
\hline $\begin{array}{l}\text { Health Risk } \\
\text { (Touch) }\end{array}$ & None & None & None & None \\
\hline $\begin{array}{c}\text { Typical } \\
\text { Operating } \\
\text { Environment }\end{array}$ & Air & Air & Encapsulated/Pump & $\begin{array}{c}\text { Aqueous } \\
\text { Solutions Only }\end{array}$ \\
\hline
\end{tabular}




\section{CHAPTER 5}

\section{FREQUENCY SHIFTING ACTUATING DIPOLE ANTENNA DESIGN}

\subsection{Operational Overview}

In this proposed battery-free sensor design, changes in temperature are conveyed through controlled and reversible changes in the operating frequency of an passive RFID tag in the UHF RFID band [13]. That is, at room temperature the sensor operates near 902 $\mathrm{MHz}$ and then the temperature increases the operating frequency of the sensor self-tunes and increases to $928 \mathrm{MHz}$. This concept is captured using the PTC (from Section 2.6) in Figure 5.1. Moreover, as the temperature returns to room temperature, the sensor automatically re-tunes to the original operating frequency of $902 \mathrm{MHz}$. Our passive (i.e., battery-free) RFID sensor can operate through numerous temperature-cycles. Hence, it functions autonomously and continuously.

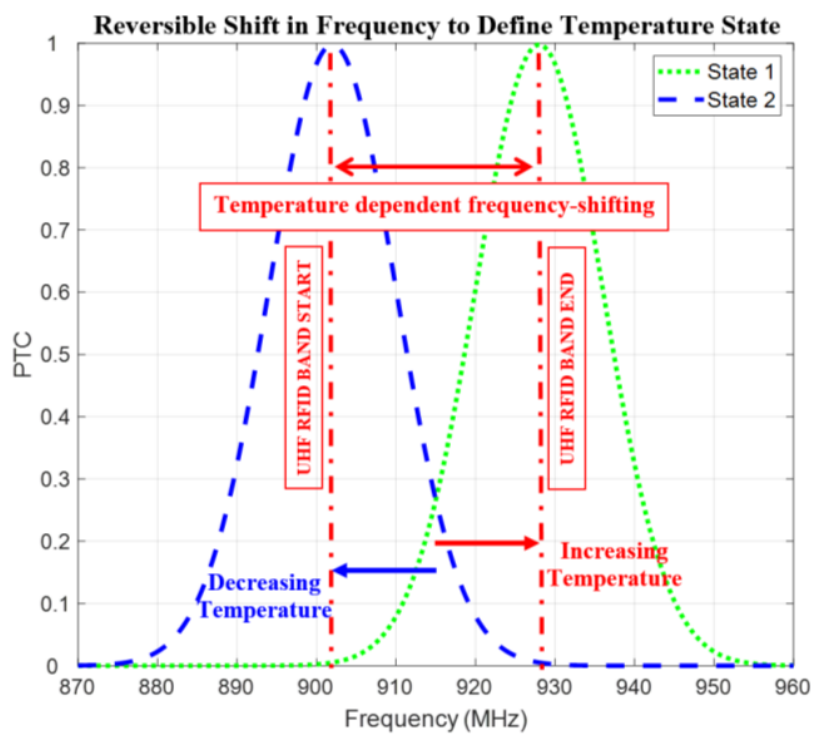

Figure 5.1. Frequency shifting capability of the developed sensor design.

\subsection{Theory of Operation}

The design consists of a planar dipole antenna made on a FR4 substrate. Just as any 
dipole antenna, this structure can be modelled as a series circuit comprising of a resistance $\left(R_{A N T}\right)$, an inductance $\left(L_{A N T}\right)$, and a capacitor $\left(C_{A N T}\right)$ [7, p. 254, pp. 314-315]. Furthermore, this antenna is customized with a T-matching network, whose design is further discussed in Section 2.7, embedded into the antenna design. This customized planar antenna is placed above a ground plane at a height, $H$, as shown in Figure 5.2.

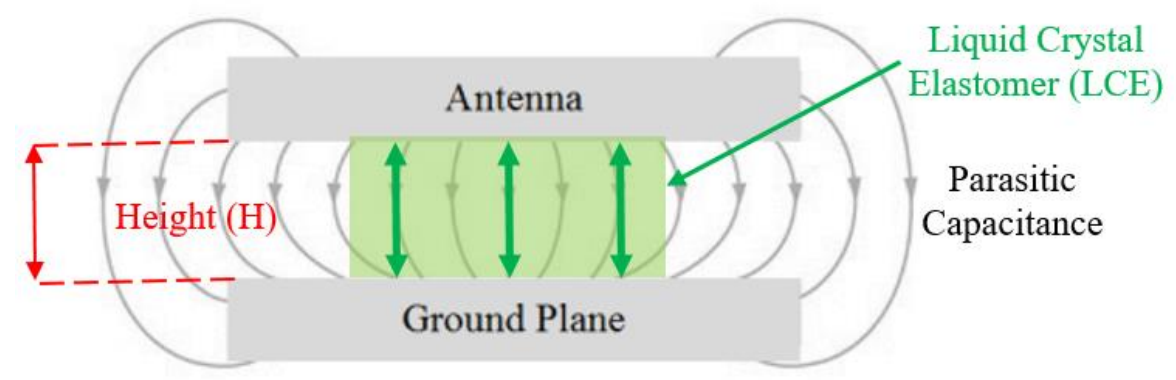

Figure 5.2. The operational frequency of the sensor is controlled through the actuation of the designed antenna above its ground plane using LCEs.

The ground plane induces a parasitic capacitance, $C_{\text {PARASITIC, }}$ which is additive to the inherent antenna capacitance, $C_{A N T}$. Moreover, the resonant frequency is inversely proportional to the parasitic capacitance as given by (5.1) [7, p. 136]:

$$
\mathrm{f}_{\text {res }}=\frac{1}{2 \pi \sqrt{\mathrm{L}_{\mathrm{ANT}}\left(\mathrm{C}_{\mathrm{ANT}}+\mathrm{C}_{\text {PARASTTIC }}\right)}}
$$

Furthermore, the parasitic capacitance is inversely proportional to the height of the antenna above the ground plane. This phenomenon is comparable to the capacitance of a parallel plate capacitor (PPC) that is given by [72, p. 240]:

$$
\mathrm{C}_{\mathrm{PPC}}=\frac{\varepsilon \mathrm{A}}{\mathrm{H}}
$$

where $A$ is the area of the plate and $\varepsilon$ is the permittivity. Therefore, since the operating frequency is inversely proportional to the parasitic capacitance, and since the parasitic 
capacitance is also inversely proportional to the height of the antenna above the ground plane, the operating frequency is directly proportional to the height above the ground plane. Finally, the height, $H$, between the antenna and the ground plane is controlled by the heatreactive LCE array as shown in Figure 4.7. As a result, the operating frequency of our sensor depends on the temperature.

The important feature in the design of this sensor is a suitable matching network to deliver a conjugate matching to the RFID IC for maximum power transfer. In Section 2.7, common matching networks typically utilized in RFID antenna design were discussed. Mainly, the T-match and the inductively-coupled loop matching networks. The configuration of these matching networks consist of feeding loops with permanent physical dimensions. Due to the concept of actuating the antenna above the ground plane, these loops will be largely influenced by the parasitic fields of the ground plane and the design dimensions would have to be altered as the height, $H$, above the ground plane is adjusted [73]. Therefore, these matching networks are impractical to fulfill our design concept.

Consequently, a clever matching network derived from the T-matching network is utilized. Specifically, the idea consists of embedding or integrating the T-match network into the planar dipole antenna [44]. This matching network is referred to as the Integrated T-Matching Network (ITMN). The ITMN has no loops; therefore, its performance is not significantly influenced by the position of the ground plane in respect to the antenna. Furthermore, the ITMN is a practical matching method, because it consists of only four design parameters [3], [13]. Since the functionality of the entire sensor revolves around the design of the ITMN, the following sections derive the formulations to determine the correct 
dimensions of the ITMN along with determining the corresponding heights, $H$, at which the desired frequency shifts will occur.

\subsection{Sensor Design}

In Section 2.7, the basic matching networks for RFID antennas were discussed. However, there still exist a large variety of more complex matching network techniques. Commercially available tags utilize various combinations of these techniques and this results in a vast array of geometrically intricate tag designs [7, pp. 305-344], [42, pp. 7480]. In fact, no set of standardized directions for designing RFID antennas exist; therefore, designing RFID tag can become an arduous task [44].

On the other hand, the design of the planar dipole antenna with the ITMN discussed here is advantageous as it requires only four physical dimensions to be determined and optimized. The planar dipole antenna with the ITMN is shown below in Figure 5.3.

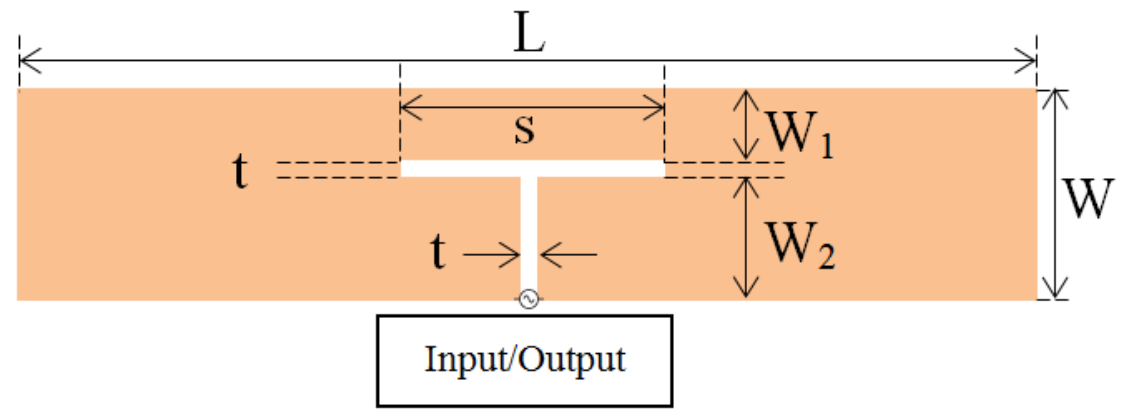

Figure 5.3. Planar dipole antenna with the ITMN.

where the dimensions to be determined are $L, W, W_{l}$, and $s$. Also, $t$ is set to $1 \mathrm{~mm}$, which provides a favorable distance at the input/output port for the RFID IC pins to make contact. A procedure and formulations will be derived to find the required design dimensions. Furthermore, since ITMN is a derivative of the traditional T-matching network that was 
described in Section 2.7, the T-matching network is utilized as a basis model to begin our calculations [13].

\subsubsection{Impedance Based Current Division Factor}

To successfully design the sensor, we also utilize simulation analysis. In Section 2.7, the current division factor, $\alpha$, was introduced in (2.17). This factor depends on the geometrical dimensions of the antenna and it was defined in Figure 2.15. In fact, the parameters that control the current division factor are shown again in Figure 5.4 for circular conductors.

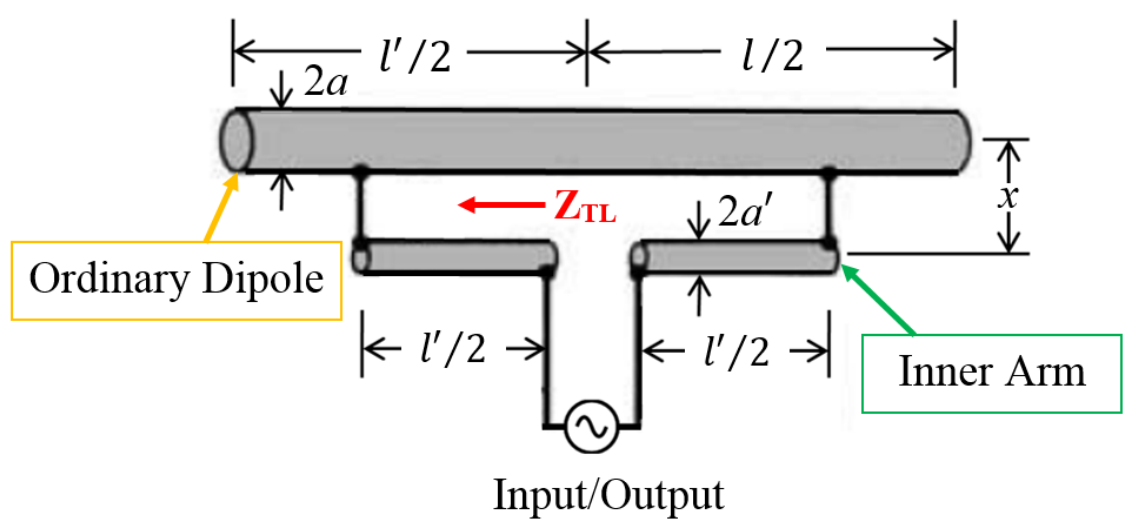

Figure 5.4. A secondary branch of conductors is added to an unmatched dipole to achieve an impedance match at the input port by adjusting the dimensions $x, l^{\prime}$, and $a^{\prime}$.

The current division factor is an important parameter, because it dictates the input impedance of the T-matching network as shown qualitatively in the transformer model of Figure 2.16 and quantitatively in (2.18). To utilize simulation analysis, a new formula for the current division factor as a function of the antenna and RFID IC impedances must be derived. In this way, the formula can be directly applied to our simulation optimization to determine the appropriate current-division-factor. To accomplish this, (2.18) is re-written as follows: 


$$
\mathrm{Z}_{\mathrm{IN}}=\mathrm{R}_{\mathrm{IN}}+\mathrm{j} \mathrm{X}_{\mathrm{IN}}=\frac{2 \mathrm{Z}_{\mathrm{TL}}\left[(1+\alpha)^{2} \mathrm{Z}_{\mathrm{A}}\right]}{2 \mathrm{Z}_{\mathrm{TL}}+(1+\alpha)^{2} \mathrm{Z}_{\mathrm{A}}}
$$

where $Z_{I N}$ should be matched to the complex conjugate of the input impedance of the RFID IC [40, p. 78]. This condition is written as follows:

$$
\mathrm{Z}_{\mathrm{IN}}=\mathrm{Z}_{\mathrm{IC}}^{*}=\mathrm{R}_{\mathrm{IC}}-\mathrm{j} \mathrm{X}_{\mathrm{IC}}
$$

The transmission line model impedance, $Z_{T L}$, is basically a shorted transmission line as shown in Figures 2.15, 2.16, and 5.4 [33, pp. 531-533]. In other words, the impedance, $Z_{T L}$, is always purely imaginary and can be written as:

$$
\mathrm{Z}_{\mathrm{TL}}=\mathrm{j} \mathrm{X}_{\mathrm{TL}}
$$

Furthermore, by substituting (5.5) and (2.10) into (5.3) and setting this equal to the right side of (5.4), the following expression is attained [13], [44]:

$$
\mathrm{Z}_{\mathrm{IN}}=\frac{2 \mathrm{j} \mathrm{X}_{\mathrm{D}}\left[(1+\alpha)^{2}\left(\mathrm{R}_{\mathrm{A}}+\mathrm{j} \mathrm{X}_{\mathrm{A}}\right)\right]}{2 \mathrm{j} \mathrm{X}_{\mathrm{D}}+(1+\alpha)^{2}\left(\mathrm{R}_{\mathrm{A}}+\mathrm{j} \mathrm{X}_{\mathrm{A}}\right)}=\mathrm{R}_{\mathrm{IC}}-\mathrm{j} \mathrm{X}_{\mathrm{IC}}
$$

In (5.6), the real-parts are set equal and after solving for $\alpha$ using a MATLAB script, the following formula for the current-division factor is obtained:

$$
\begin{gathered}
\alpha=-\frac{\mathrm{R}_{\mathrm{A}}^{2} \mathrm{R}_{\mathrm{IC}}+\mathrm{R}_{\mathrm{IC}} \mathrm{X}_{\mathrm{A}}^{2}-\sqrt{\left(\mathrm{R}_{\mathrm{A}} \mathrm{R}_{\mathrm{IC}}\left(\mathrm{R}_{\mathrm{A}}^{2}+\mathrm{X}_{\mathrm{A}}^{2}\right)\left(\mathrm{R}_{\mathrm{IC}}{ }^{2}+\mathrm{X}_{\mathrm{IC}}{ }^{2}\right)\right)}}{\mathrm{R}_{\mathrm{IC}} \mathrm{R}_{\mathrm{A}}^{2}+\mathrm{R}_{\mathrm{IC}} \mathrm{X}_{\mathrm{A}}^{2}} \\
=\sqrt{\frac{\mathrm{R}_{\mathrm{A}}\left(\mathrm{R}_{\mathrm{IC}}{ }^{2}+\mathrm{X}_{\mathrm{IC}}{ }^{2}\right)}{\mathrm{R}_{\mathrm{IC}}\left(\mathrm{R}_{\mathrm{A}}^{2}+\mathrm{X}_{\mathrm{A}}^{2}\right)}}-1
\end{gathered}
$$

This equation is significant because it is a function of the antenna and RFID IC input complex impedances and it can be adapted into simulation optimization and analysis. Additionally, it indirectly shows that $\alpha$ depends on the geometry of the planar dipole 
antenna. In other words, when this formula is utilized in simulation, various dimensions of the planar antenna design can be investigated; particularly, the length, $L$, and width, $W$, (see Figure 5.3) should be determined to achieve the desired value of $\alpha$, which can be utilized for calculating the remaining dimensions in Figure 5.3.

\subsubsection{Geometry Based Current Division Factor}

The geometry based current-division-factor from (2.17) assumes cylindrical conductors, as shown in Figure 5.4. However, the design of the dipole antenna from Figure 5.3 is designed using very thin flat-planar conductors. To correctly consider the antenna characteristics and utilize (2.17), an equivalent circular conductor must be utilized to replace the non-circular cross-section of the flat-planar conductors. To achieve this, the Effective Equivalent Radius (EER) as depicted in Figure 5.5 is utilized [33, p. 514].

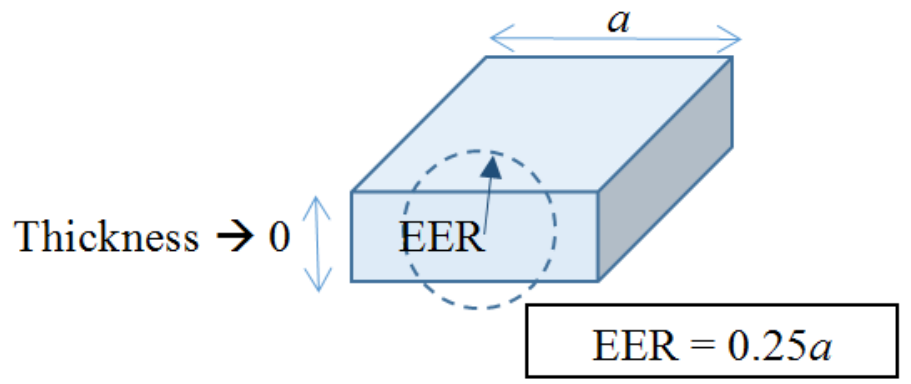

Figure 5.5. Electrical equivalent radius (EER) of a flat planar conductor. where $a$ represents the width of the flat conductor and the EER is simply $a / 4$.

The width, $W$, of the antenna structure from Figure 5.3 is dissected into three sections of flat-planar conductors. Each segment's EER is calculated based on the physical dimension of the flat-conductor segment. Figure 5.6 shows the updated antenna design using the corresponding equivalent-effective-radii, EER ${ }_{\mathrm{w} 1}, \mathrm{EER}_{\mathrm{t}}$, and EER $\mathrm{W}_{2}$. 


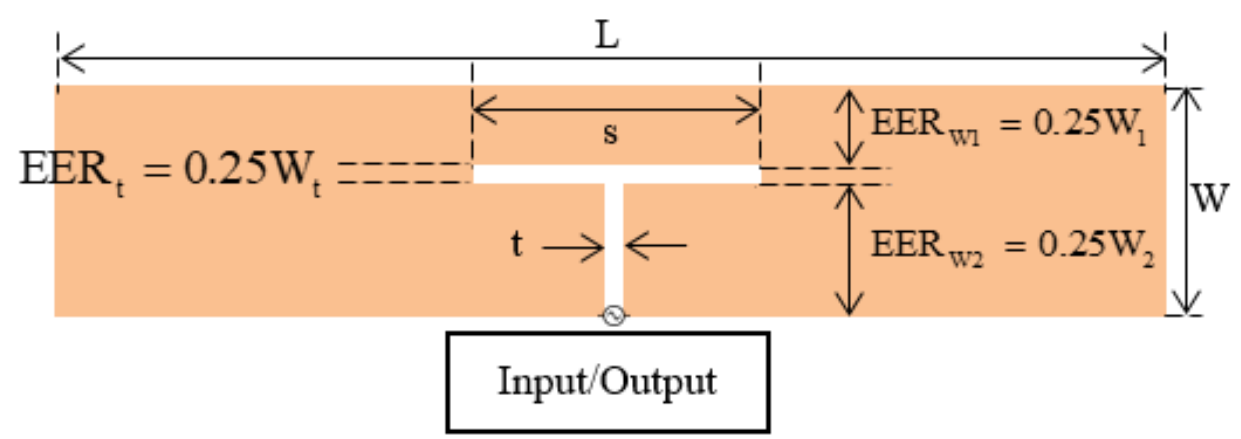

Figure 5.6. Planar antenna design with the ITMN and the EERs defined for the flat conductors.

Based on Figure 5.4, when calculating the dimension $x$, the EER of the center spacing in Figure 5.6 is doubled, i.e., $2 \mathrm{EER}_{\mathrm{t}}$, since the EER represents an equivalent radius and not the diameter [13]. Since the antenna structure is now correctly re-defined using the EER, the following dimensional-ratios from (i.e., $x / a^{\prime}$ and $\left.a / a^{\prime}\right)$ from (2.17) can now be written as follows:

$$
\begin{gathered}
\frac{\mathrm{x}}{\mathrm{a}^{\prime}}=\frac{\mathrm{EER}_{\mathrm{W} 1}+2 \mathrm{EER}_{\mathrm{t}}+\mathrm{EER}_{\mathrm{W} 2}}{\mathrm{EER}_{\mathrm{W} 2}}=\frac{\mathrm{W}_{1}+2 \mathrm{t}+\mathrm{W}_{2}}{\mathrm{~W}_{2}} \\
\frac{\mathrm{a}}{\mathrm{a}^{\prime}}=\frac{\mathrm{EER}_{\mathrm{W} 1}}{\mathrm{EER}_{\mathrm{W} 2}}=\frac{\mathrm{W}_{1}}{\mathrm{~W}_{2}}
\end{gathered}
$$

Furthermore, these ratios can now be incorporated into (2.17), which gives the appropriate current division factor for the flat-planar antenna design of Figure 5.6 as follows:

$$
\alpha=\frac{\ln \left(\frac{\mathrm{x}}{\mathrm{a}^{\prime}}\right)}{\ln \left(\frac{\mathrm{x}}{\mathrm{a}^{\prime}}\right)-\ln \left(\frac{\mathrm{a}}{\mathrm{a}^{\prime}}\right)}=\frac{\ln \left(\frac{\mathrm{W}_{1}+2 \mathrm{t}+\mathrm{W}_{2}}{\mathrm{~W}_{2}}\right)}{\left(\ln \left(\frac{\mathrm{W}_{1}+2 \mathrm{t}+\mathrm{W}_{2}}{\mathrm{~W}_{2}}\right)-\ln \left(\frac{\mathrm{W}_{1}}{\mathrm{~W}_{2}}\right)\right)}
$$

In the right side of (5.10) the term: $W_{1}+2 t+W_{2}$ appears twice. According to Figure 5.3, the width, $W$, is equal to: $W=W_{1}+t+W_{2}$. Moreover, since the distance $t$ is relatively 
smaller than the dimensions $W_{l}$ and $W_{2}$, the following expression may be used for the width $W$ with minimal error: $W=W_{1}+2 t+W_{2}$. In making this substitution, (5.10) becomes:

$$
\alpha=\ln \left(\frac{\mathrm{W}}{\mathrm{W}_{2}}\right) /\left(\ln \left(\frac{\mathrm{W}}{\mathrm{W}_{2}}\right)-\ln \left(\frac{\mathrm{W}_{1}}{\mathrm{~W}_{2}}\right)\right)
$$

Due to this substitution, (5.11) is now structured so that a concise and simple formula can be obtained for the width $W_{l}$, (refer to Figure 5.3) as follows [13]:

$$
\mathrm{W}_{1}=\left(\mathrm{W}\left(\frac{\mathrm{W}_{2}}{\mathrm{~W}}\right)^{\frac{1}{\alpha}}\right)
$$

Additionally, from Figure 5.3, the width $W_{2}$ controls the vertical positioning of the ITMN. For a pragmatic design and to further simplify (5.12) to a function of a single variable, the width $W_{2}$ is restricted to half the width of the antenna, $W$; that is, $W_{2}=W / 2$. Moreover, for easy and efficient calculation of the antenna dimensions, the width $W_{l}$ is always assumed to be an integer. Using both these simplification concepts in (5.12), the width $W_{l}$ is reduced to a simplistic formula as follows [13]:

$$
\mathrm{W}_{1}=\operatorname{integer}\left(\mathrm{W}\left(\frac{1}{2}\right)^{\frac{1}{\alpha}}\right)
$$

Also, from Figure 5.3 the formula for the width $W_{2}$ is easily obtained follows:

$$
\mathrm{W}_{2}=\mathrm{W}-\mathrm{t}-\mathrm{W}_{1}
$$

As mentioned in Section 5.3, the width, $t$, is set to $1 \mathrm{~mm}$ as it is the appropriate spacing to attach the RFID IC. In summary, (5.7), (5.13) and (5.14) are the important design equations needed to design the dipole planar antenna with the ITMN. 


\subsubsection{Design Process}

To begin the sensor design, an ordinary planar dipole antenna is simulated using FR4 substrate to operate at $928 \mathrm{MHz}$ in ANSYS HFSS. This antenna design incorporates the FR4 substrate as it provides a stable support for the RFID IC and improves the overall structural integrity of the antenna. Additionally, a square ground plane is placed under the antenna with side-lengths of $150 \mathrm{~mm}$. The antenna is then positioned $8 \mathrm{~mm}$ above this ground plane as shown in Figure 5.7 [13].

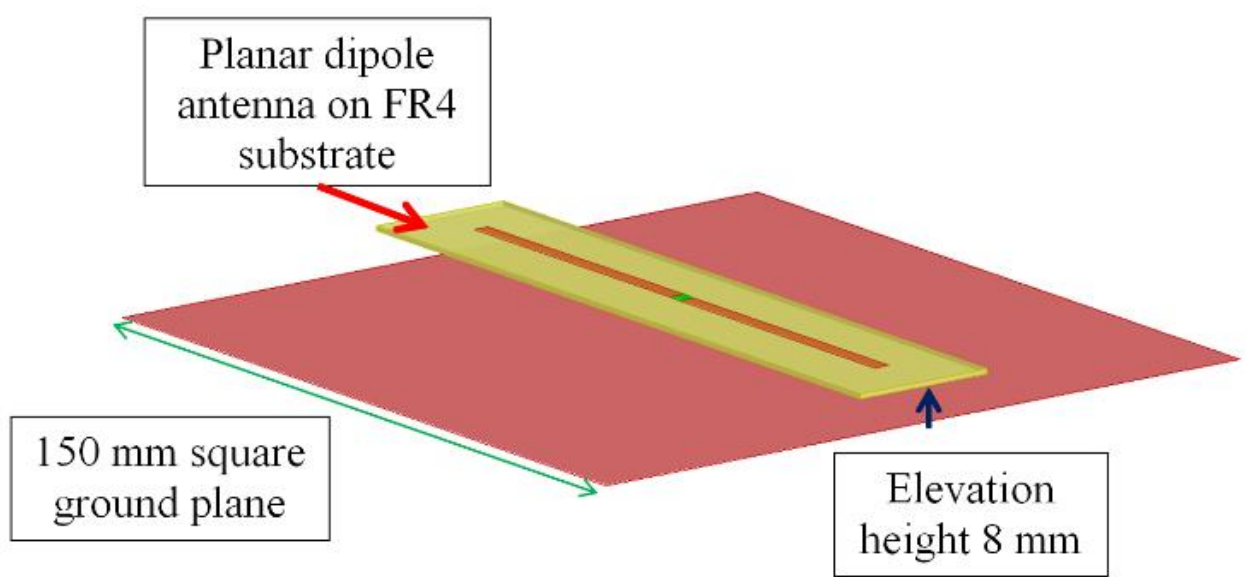

Figure 5.7. ANSYS HFSS simulation model of the planar dipole antenna design on FR4 substrate over the ground plane.

Subsequently, the width, $W$, of the antenna is swept from $10 \mathrm{~mm}$ to $60 \mathrm{~mm}$ in intervals of $10 \mathrm{~mm}$. Also, the length, $L$, of the antenna is varied from $80 \mathrm{~mm}$ to $180 \mathrm{~mm}$. For each antenna model corresponding to each width, $W$, the input impedance is simulated as the length, $L$, is swept. Subsequently, (5.7) is used to calculate the current-division-factor, $\alpha$, in simulation with the RFID IC impedance $Z_{\text {IC }}$ of $18-164 j$. The resulting plot of $\alpha$ for each antenna width is depicted in Figure 5.8 below. 


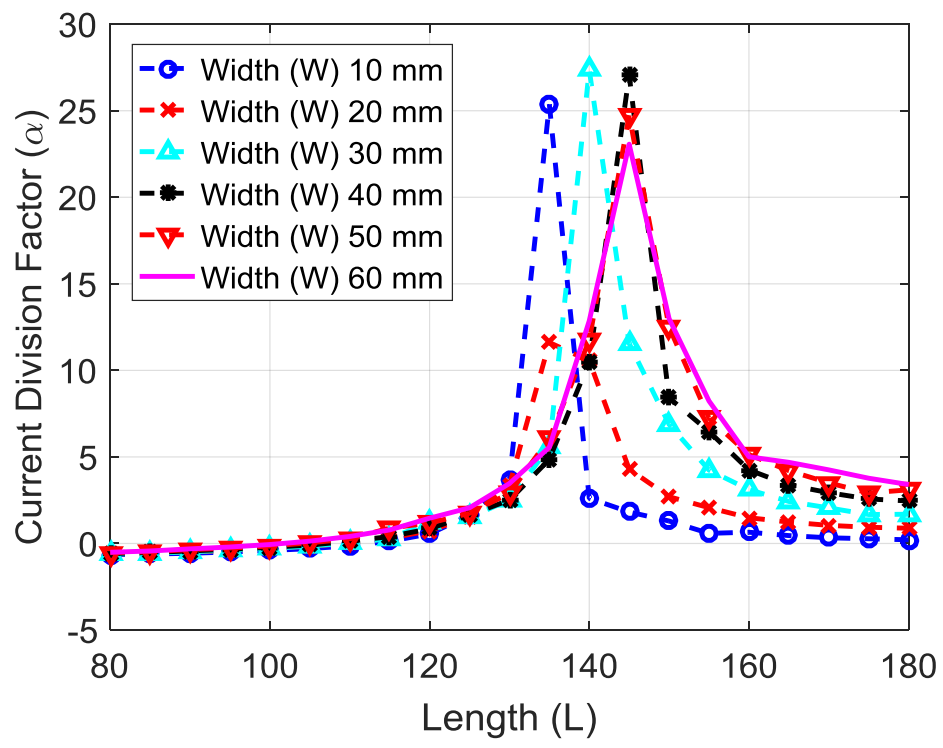

Figure 5.8. Current division factor, $\alpha$, for different dipole lengths and widths.

This current-division-factor plot defines a combinational solution of $\alpha, W$, and $L$ for the antenna design depicted in Figure 5.3. In other words, the length, $L$, and width, $W$, of the antenna are now known. Furthermore, using the width, $W$, and the current-divisionfactor, $\alpha$, in (5.13) and (5.14) the remaining design dimension of the widths $W_{l}$ and $W_{2}$ can now easily be determined, respectively [13]. Through testing various values of the length $L$, the value of $\alpha$ associated with realizable values of the width $W_{l}$ are obtained at the antenna length of $120 \mathrm{~mm}$. Furthermore, only the antenna designs with the three smallest widths of $10 \mathrm{~mm}, 20 \mathrm{~mm}$, and $30 \mathrm{~mm}$ (designs A, B, and C; respectively,) are chosen for evaluation as they provide the designs with the lightest weights [13].

Thus far, a methodology to provide all the dimensions of the antenna design from Figure 5.3 are provided except for the length, $s$. To determine this parameter, simulation analysis is utilized. Specifically, the current-division-factor, $\alpha$, and the width, $W$, are first determined for the length, $L$, of $120 \mathrm{~mm}$ for each width corresponding to designs $\mathrm{A}, \mathrm{B}$, and 
$\mathrm{C}$ from Figure 5.8. Each design is then simulated with the antenna is elevated $8 \mathrm{~mm}$ above the ground plane as shown in Figure 5.9.

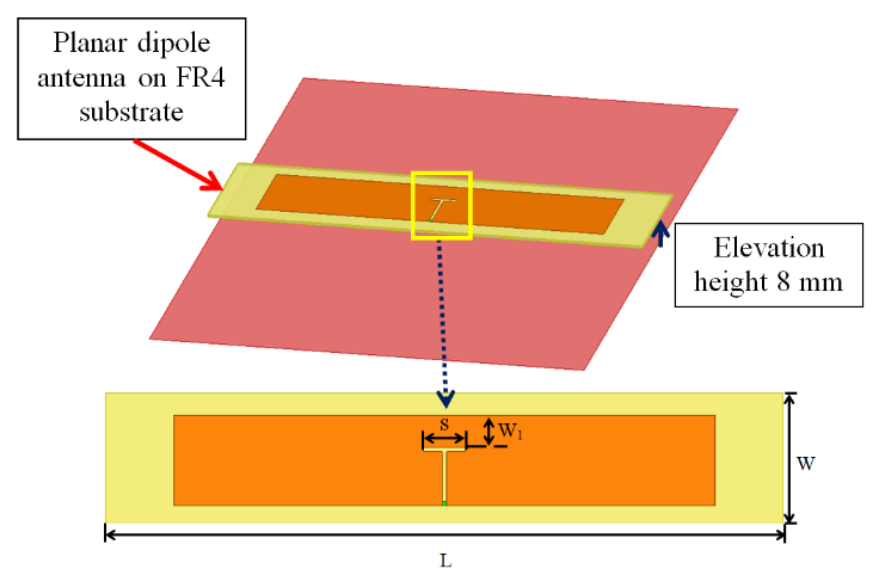

Figure 5.9. ANSYS HFSS simulation model of the planar antenna design with the ITMN.

The length, $s$, is then swept until the operating frequency of the antenna is $930 \mathrm{MHz}$, slightly higher than the maximum operating frequency in the RFID UHF band of $928 \mathrm{MHz}$. This setup serves as a starting point. That is, since the height is directly proportional to the operating frequency, the antenna is now incrementally lowered and the heights that correspond to the operating frequencies of $928 \mathrm{MHz}$ and $902 \mathrm{MHz}$ are recorded. At this point all dimensions have been determined and the design is complete. For designs A, B, and $\mathrm{C}$, all the determined parameters are summarized in Table 5.1 below.

Table 5.1. Final design dimensions with the heights that correspond to operating frequencies.

\begin{tabular}{|c|c|c|c|c|c|c|c|}
\hline Design & $\begin{array}{c}\mathrm{W} \\
(\mathrm{mm})\end{array}$ & $\begin{array}{c}\mathrm{L} \\
(\mathrm{mm})\end{array}$ & $\alpha$ & $\begin{array}{c}\mathrm{W}_{1} \\
(\mathrm{~mm})\end{array}$ & $\begin{array}{c}\mathrm{W}_{2} \\
(\mathrm{~mm})\end{array}$ & $\mathrm{s}(\mathrm{mm})$ & $\begin{array}{c}\text { Heights } \\
902 \mathrm{MHz} \\
928 \mathrm{MHz}\end{array}$ \\
\hline $\mathrm{A}$ & 10 & 120 & 0.55 & 2 & 7 & 9.8 & $\begin{array}{c}3.6 \mathrm{~mm} \\
7.2 \mathrm{~mm}\end{array}$ \\
\hline $\mathrm{B}$ & 20 & 120 & 0.74 & 7 & 12 & 9.4 & $\begin{array}{c}3.6 \mathrm{~mm} \\
6.8 \mathrm{~mm}\end{array}$ \\
\hline $\mathrm{C}$ & 30 & 120 & 1.23 & 17 & 12 & 17.7 & $\begin{array}{c}2.8 \mathrm{~mm} \\
6.8 \mathrm{~mm}\end{array}$ \\
\hline
\end{tabular}


It is important to recall that this sensor operates by conveying changes in temperature through the controlled shift in the operating frequency. Hence, to clearly convey to the operator that a temperature threshold has been crossed, this sensor is designed to have the largest shift in the operating frequency within the RFID UHF band.

In summary, to design the planar dipole antenna with the ITMN, the currentdivision-factor, $\alpha$, is first plotted using simulation and (5.7). Moreover, $\alpha$ is plotted versus the length, $L$, of the antenna, for a given width, $W$, of the antenna. Secondly, (5.13) and (5.14) are then used to determine the widths $W_{l}$ and $W_{2}$, respectively. Finally, the length, $s$, from Figure 5.9, is varied until the desired operating frequency is reached.

\subsection{Optimum Design Selection}

Each of the designs from Table 5.1 are simulated as shown in Figure 5.9 at their corresponding heights. Specifically, (2.14) is utilized in our simulations to plot the Power Transmission Coefficient (PTC), $\tau$, for each design. Our results are depicted in Figure 5.10.

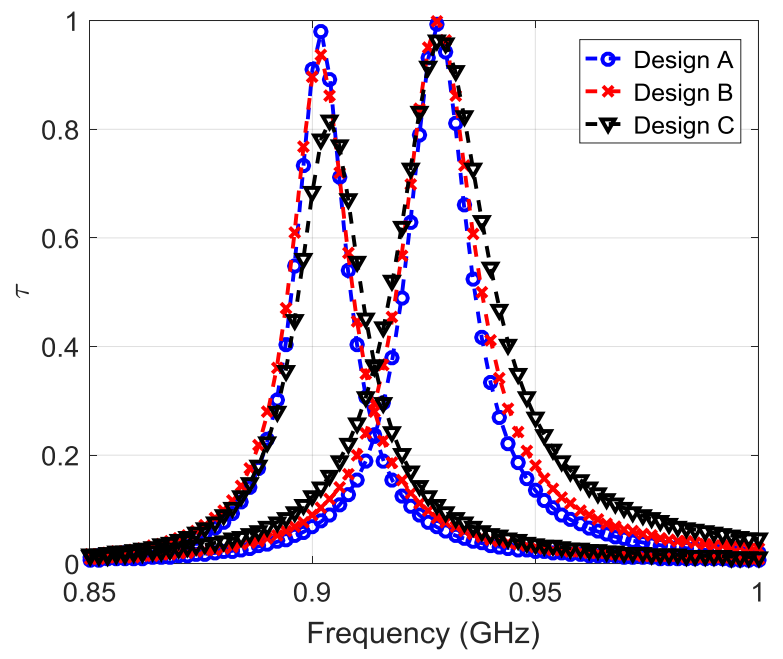

Figure 5.10. Power transmission coefficient vs. frequency for designs A, B, and C, from Table. IV. This plot depicts the performance of each design at the two operating frequencies of $902 \mathrm{MHz}$ and $928 \mathrm{MHz}$. 
From this figure, it is apparent that the smaller and larger elevation heights (for each design) above the ground plane correspond to the operating frequencies of $902 \mathrm{MHz}$ and $928 \mathrm{MHz}$, respectively. Also, Designs A and B display better performance when compared to Design C since the PTC is larger at both the operating frequencies.

Furthermore, Designs A and B are similar in performance in regards their PTC. However, from Table 5.1, the difference in the two designs is in the height displacement required for each design to transition operating frequencies from $902 \mathrm{MHz}$ to $928 \mathrm{MHz}$. In other words, for Design A, the antenna must travel from $3.6 \mathrm{~mm}$ to $7.2 \mathrm{~mm}$ above the ground plane to transition between the two operating frequencies, this is a height displacement of $3.6 \mathrm{~mm}$. On the other hand, Design B, must travel from $3.6 \mathrm{~mm}$ to $6.8 \mathrm{~mm}$ above the ground plane, which is height displacement of $3.2 \mathrm{~mm}$. In Section 4.4, it was mentioned that the maximum displacement of the LCE array is $4 \mathrm{~mm}$ at approximately $160^{\circ}$ C. Therefore, the smaller height displacement of Design B is favorable as this translates to less heat stimulus required to excite the LCE. This extends the lifespan of the LCE actuator by avoiding overheating, overstretching, and fatigue. For this reason, Design B is chosen as the optimum design and chosen for fabrication in this research [13].

\subsubsection{Sensor Radiation Performance}

Since this is a wireless sensor, it is important to analyze its radiation characteristics. Particularly, we calculate the radiation pattern, gain, and radiation efficiency of Design B from Table 5.1 at both heights above the ground plane. The radiation pattern and gain of our design are similar for both operating states. That is, for when the antenna is $3.6 \mathrm{~mm}$ above the ground plane (operating at $902 \mathrm{MHz}$ ) and when the antenna is $6.8 \mathrm{~mm}$ above the 
ground plane (operating at $928 \mathrm{MHz}$ ). Moreover, the radiation pattern is similar to the one of a typical dipole antenna; however, in this case the radiation pattern is directed toward the half-plane due to the presence of the ground plane that behaves as a reflector. Finally, in both cases the maximum gain is $3.7 \mathrm{dBi}$ and the $3 \mathrm{D}$ radiation pattern is depicted below in Figure 5.11.

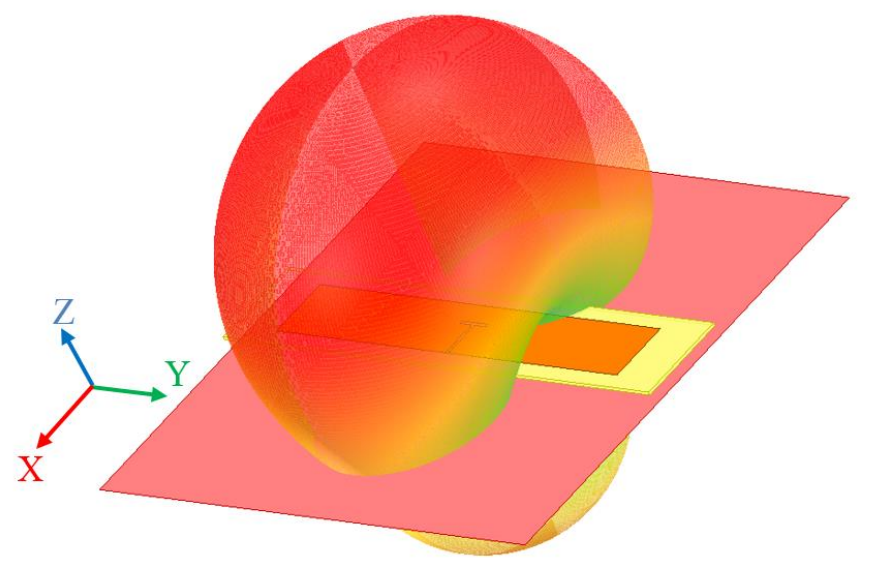

Figure 5.11. Simulated 3D radiation pattern of the designed sensor. The radiation pattern and maximum gain are similar for both cases when the antenna is elevated above the ground plane to $3.6 \mathrm{~mm}$ (operating at $902 \mathrm{MHz}$ ) and when the antenna is elevated to

$6.8 \mathrm{~mm}$ above the ground plane (operating at $928 \mathrm{MHz}$ ).

Furthermore, the corresponding polar plots for the $\mathrm{XY}, \mathrm{YZ}$, and $\mathrm{XZ}$ principal planes along with the corresponding gain are shown in Figures 5.12, 5.13, and 5.14, respectively. 


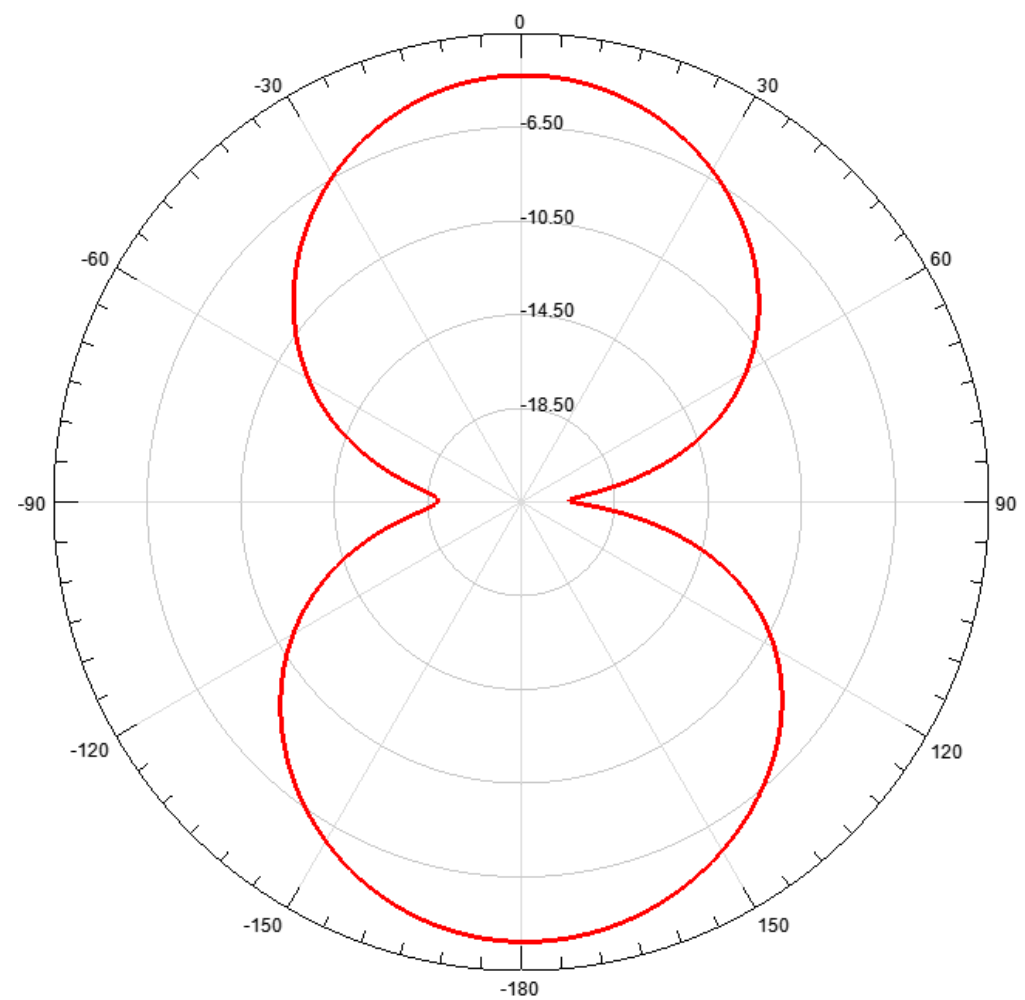

Figure 5.12. HFSS polar gain plot for the XY principal plane.

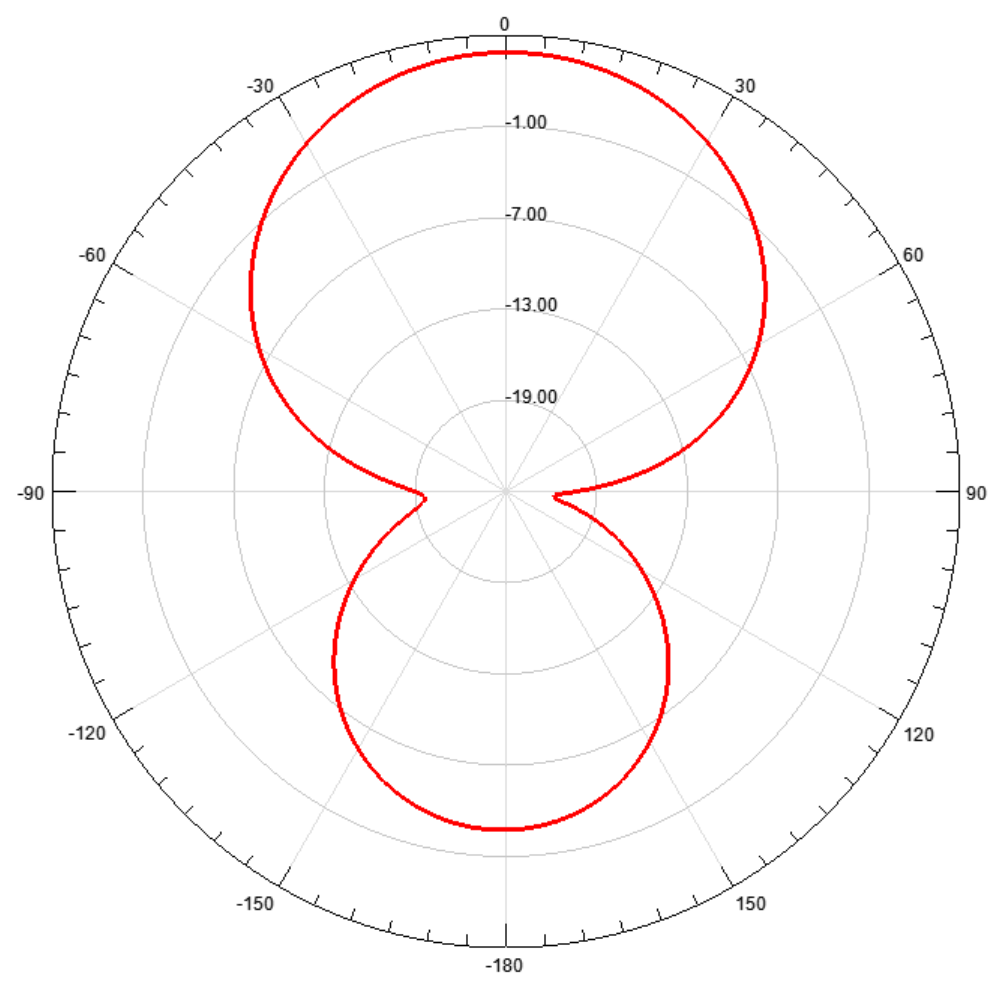

Figure 5.13. HFSS polar gain plot for the YZ principal plane. 


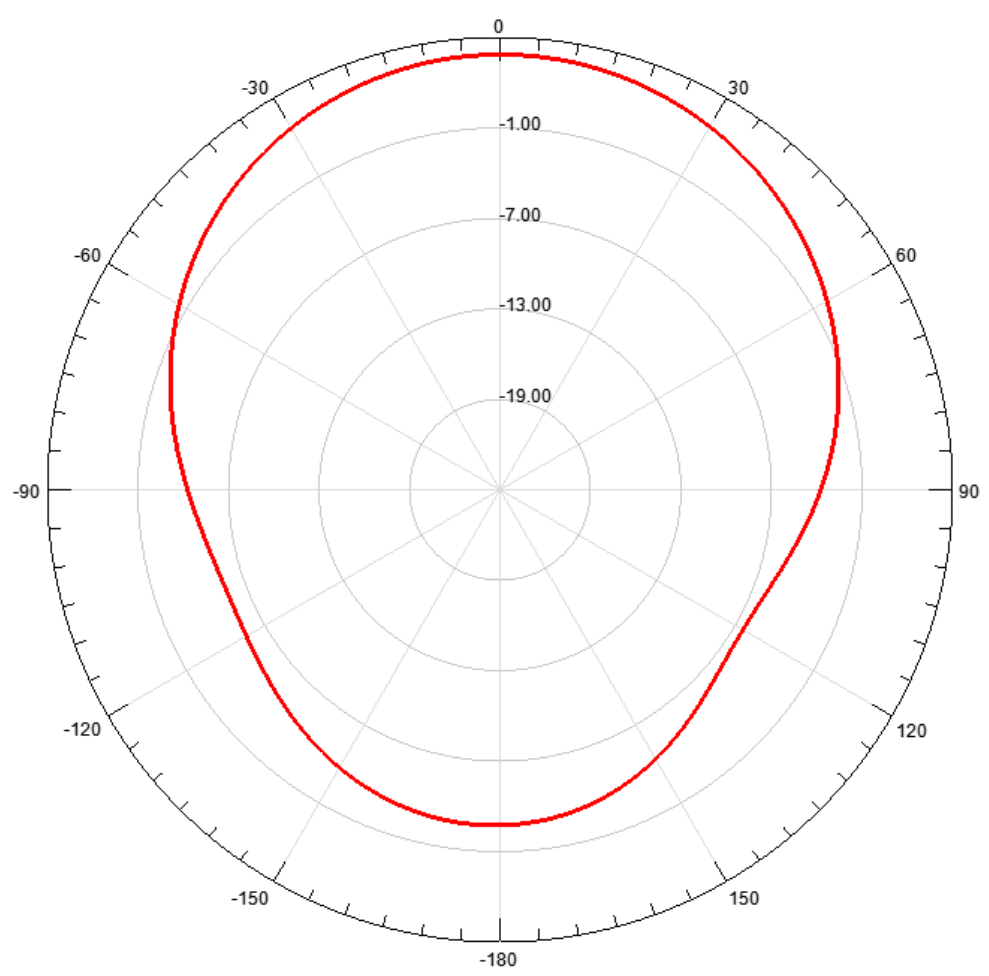

Figure 5.14. HFSS polar gain plot for the XZ principal plane.

Since the sensor design consists of a horizontal dipole in close-proximity to a ground plane, an adverse effect to the radiation is present. To better understand this effect, the ground plane can be substituted by a virtual image of the horizontal dipole mirrored across the plane of the ground plane and with the currents flowing in the opposite direction [33, pp. 184-187]. As a result, the potential that reaches an observation point in the farfield is partially cancelled due to this image. As the horizontal dipole gets closer to the ground plane, the radiation completely diminishes [7, pp. 338-339]. Therefore, it is important to gauge the performance of the developed sensor using the radiation efficiency or the ratio of the power radiated to the power fed at its input terminals [72, pp. 670-671]. In the case when the horizontal dipole is $3.6 \mathrm{~mm}$ (operating at $902 \mathrm{MHz}$ ) above from the 
ground plane, its radiation efficiency is $60 \%$. Additionally, in the case where the dipole is $6.8 \mathrm{~mm}$ above the ground plane (operating at $928 \mathrm{MHz}$ ), its radiation efficiency is $70 \%$.

Finally, the sensor is designed to be perfectly matched to the RFID IC load in both cases. Thus, the mismatch losses are negligible. Also, the antenna is modeled using PEC boundary conditions (where $\sigma=\infty$ ). Therefore, the losses due to skin-resistance are zero [72, pp. 461-464]. Furthermore, the FR4 dielectric substrate has a small loss tangent (i.e., $\sigma \ll \omega \varepsilon)$; hence, it behaves as a lossless dielectric [72, p. 459]. In conclusion, the efficiency of our RFID antenna is primarily influenced by the presence of the ground plane and represents the extent to which the antenna radiates (or the portion of antenna impedance which radiates).

\subsection{Sensor Prototyping and Performance Testing}

The antenna Design B (see Table 5.1) was fabricated on a single-sided FR4 substrate board using an LPKF S103 micro-milling machine. The prototype is shown in Figure 5.15.

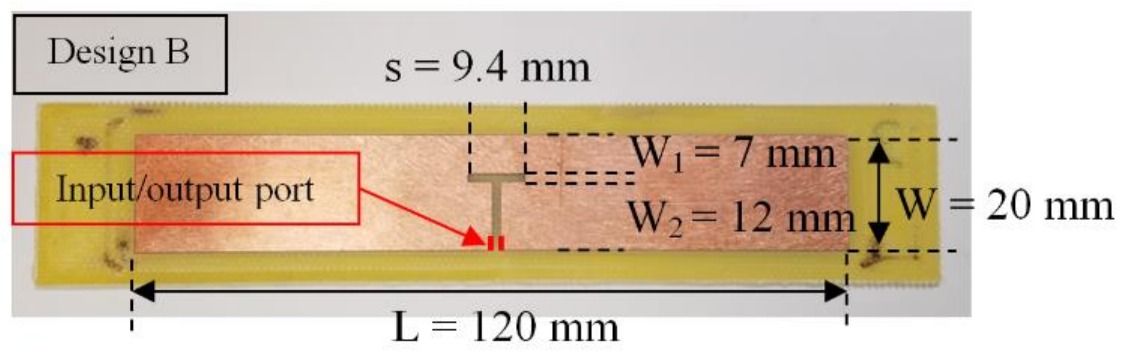

Figure 5.15. Prototype of planar antenna design with ITMN.

To verify the simulation results and confirm the design methodology, the performance of this fabricated design is tested by measuring its input impedance. Hence, the RFID IC is not installed yet. To measure the input impedance of this design a specialized differential probe must be utilized because this dipole-planar antenna is a balanced type of antenna. 
Specifically, dipoles are antennas that consists of symmetrical structures, which are driven by opposite currents. Also, the dipole antenna operates in reference to itself as opposed to a single-ended or unbalanced antenna that operates with reference to a groundplane [7, pp. 279-281]. For these reasons, the dipole antenna is considered a balanced antenna. On the other hand, the coaxial cable which is commonly used to take RF measurements is an asymmetrical transmission line since the inner and outer conductors are not identical. Thus, a coaxial cable is an unbalanced transmission line.

Due to the fact that the coaxial cable is an unbalanced medium and the dipole antenna is a balanced structure, if the coaxial cable is connected to this antenna a netcurrent flow to the ground will result on the outer surface of the outer conductor of the coaxial cable [13], [33, pp. 539-541], [74]. Thus, the input impedance of the prototype design cannot be measured through the conventional method of using a coaxial type cable connected to a VNA and taking an $\mathrm{S}_{11}$ measurement.

The solution to measuring the input impedance of the balanced planar dipole antenna is discussed in [75] and [76]. The technique involves bisecting the antenna at the input/output port, refer to Figure 5.15, into two individual ports in reference to a newly introduced ground plane without any disturbance to the current distribution on the antenna. A diagram depicting this notion is shown in Figure 5.16. 


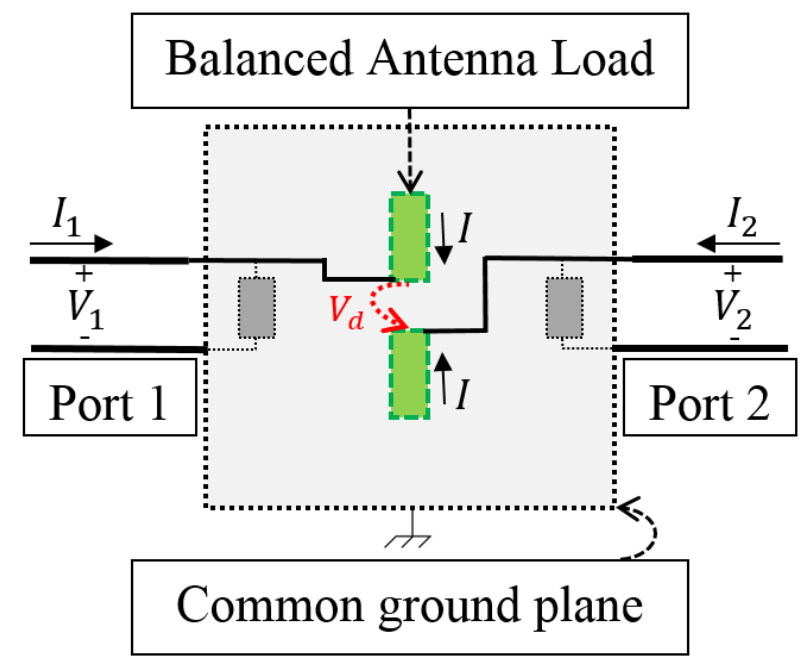

Figure 5.16. Balanced antenna measuring concept.

The input impedance is then derived based on the differential voltage, $V_{d}$, between the two ports of the network [13], [75]. In detail, the differential voltage, $V_{d}$, is defined as:

$$
\mathrm{V}_{\mathrm{d}}=\mathrm{V}_{2}-\mathrm{V}_{1}
$$

Therefore, the differential impedance is defined as:

$$
\mathrm{Z}_{\mathrm{d}}=\frac{\mathrm{V}_{\mathrm{d}}}{\mathrm{I}}=\frac{\mathrm{V}_{2}-\mathrm{V}_{1}}{\mathrm{I}}
$$

where $V_{1}$ and $V_{2}$ can be written as follows based on two-port network theory [40, pp. 174175]:

$$
\begin{aligned}
& \mathrm{V}_{1}=\mathrm{Z}_{11} \mathrm{I}_{1}+\mathrm{Z}_{12} \mathrm{I}_{2} \\
& \mathrm{~V}_{2}=\mathrm{Z}_{21} \mathrm{I}_{1}+\mathrm{Z}_{22} \mathrm{I}_{2}
\end{aligned}
$$

Since the antenna is symmetrical, $I_{1}=I$ and $I_{2}=-I$, the differential voltage now becomes:

$$
\mathrm{V}_{\mathrm{d}}=\mathrm{V}_{2}-\mathrm{V}_{1}=\left(\mathrm{Z}_{11}-\mathrm{Z}_{21}-\mathrm{Z}_{12}+\mathrm{Z}_{22}\right) \mathrm{I}
$$

the differential impedance then becomes: 


$$
\mathrm{Z}_{\mathrm{d}}=\frac{\mathrm{V}_{\mathrm{d}}}{\mathrm{I}}=\frac{\mathrm{V}_{2}-\mathrm{V}_{1}}{\mathrm{I}}=\left(\mathrm{Z}_{11}-\mathrm{Z}_{21}-\mathrm{Z}_{12}+\mathrm{Z}_{22}\right)
$$

Finally, converting the Z- parameters to S-parameters [40, p. 192]:

$$
Z_{\text {in }}=\frac{2 Z_{0}\left(1-S_{11} S_{22}+S_{12} S_{21}-S_{12}-S_{21}\right)}{\left(1-S_{11}\right)\left(1-S_{22}\right)-S_{21} S_{12}}
$$

This equation provides the final formula for measuring the input impedance as a function of the measured S-parameters.

To perform this differential measurement correctly, a custom differential probe is constructed. The probe consists of two SMA coaxial cables that are shorted together by soldering the outer conductors of each cable to each other. Moreover, the inner conductors of the cables are exposed but left untouched in an open-circuit arrangement. The shorted outer conductors serve as the new common ground plane in reference to each of the two inner conductors in which the differential voltages are measured from, as shown in Figure 5.16. The fabricated test fixture is shown in Figure 5.17.

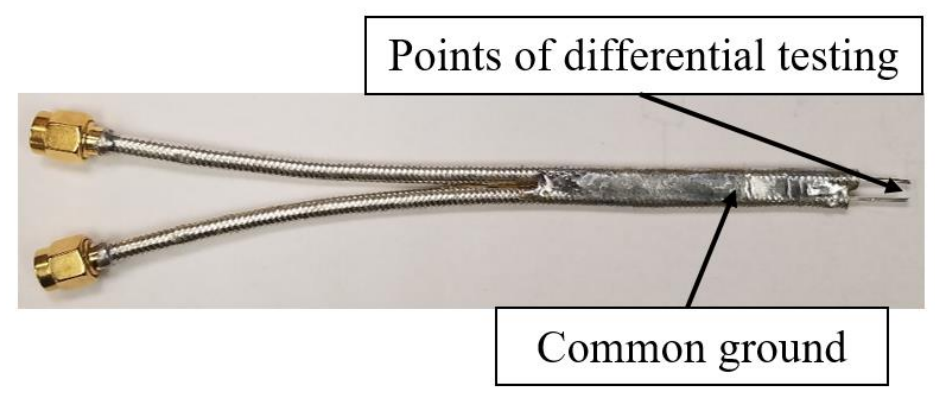

Figure 5.17. Fabricated differential probe for balanced antenna impedance measurements.

The Keysight E5061B VNA was used to conduct our measurements. A pair of SMA coaxial cables are first connected to the VNA and their ports are calibrated using the typical 2-port calibration. Unfortunately, this common calibration technique cannot account for the electrical characteristics of the differential probe. Consequently, after calibrating the 
cables, the differential probe is connected to the cables and using the Auto Port Extension capability of the VNA, the electrical length of the test probe is de-embedded using the "open-circuit" option since the inner conductors in an open configuration (refer to Figure 5.17) [13]. This setup procedure is summarized in Figure 5.18.

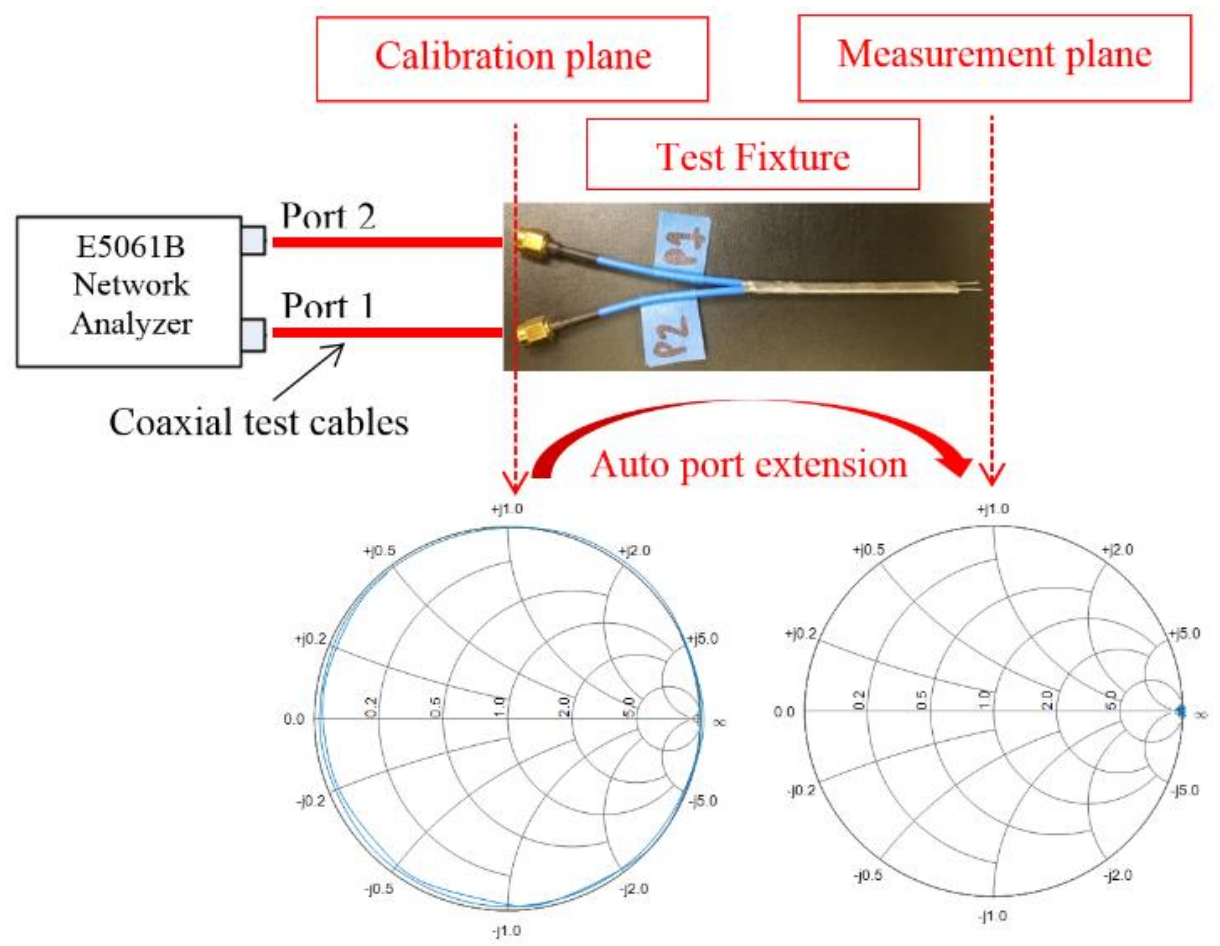

Figure 5.18. Calibration process of differential probe.

Furthermore, to confirm that the VNA has been properly calibrated with the test fixture, one can view the response of the VNA (i.e., $S_{11}$ or $S_{22}$ ) on a Smith chart with the differential probe connected. Specifically, the left Smith chart in Figure 5.18 shows the response of the differential probe before the use of the auto-port correction. Once the auto-port correction is applied (see the right Smith chart in Figure 5.18) the VNA is calibrated at the ends of the differential probe [13], [75].

The antenna design from Figure 5.15 is soldered to the inner terminals of the test fixture. The antenna is then placed at the corresponding heights for Design B above the 
ground plane from Table 5.1, i.e., at $3.6 \mathrm{~mm}$ to operate at $902 \mathrm{MHz}$ and $6.8 \mathrm{~mm}$ to operate at $928 \mathrm{MHz}$. A wooden jig was used to hold the antenna and test fixture in place. A picture of the test setup is shown in Figure 5.19.

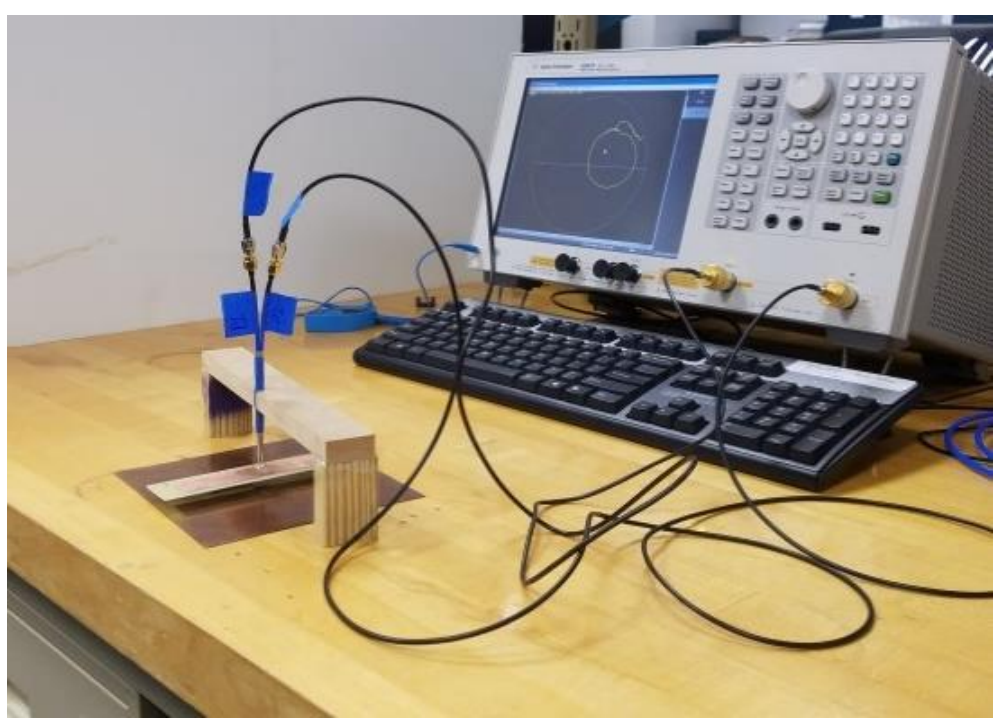

Figure 5.19. Impedance measurement setup with differential probe and VNA.

Additionally, for each position above the ground plane, the full two-port S-parameters were taken. Using post-processing and equation (5.21), the input impedance is calculated. Furthermore, this measured input impedance is compared with the simulation results for both positions above the ground plane. These results are depicted in Figures 5.20 and 5.21 for both positions of the antenna above the ground plane, i.e., $3.6 \mathrm{~mm}$ and $6.8 \mathrm{~mm}$. 


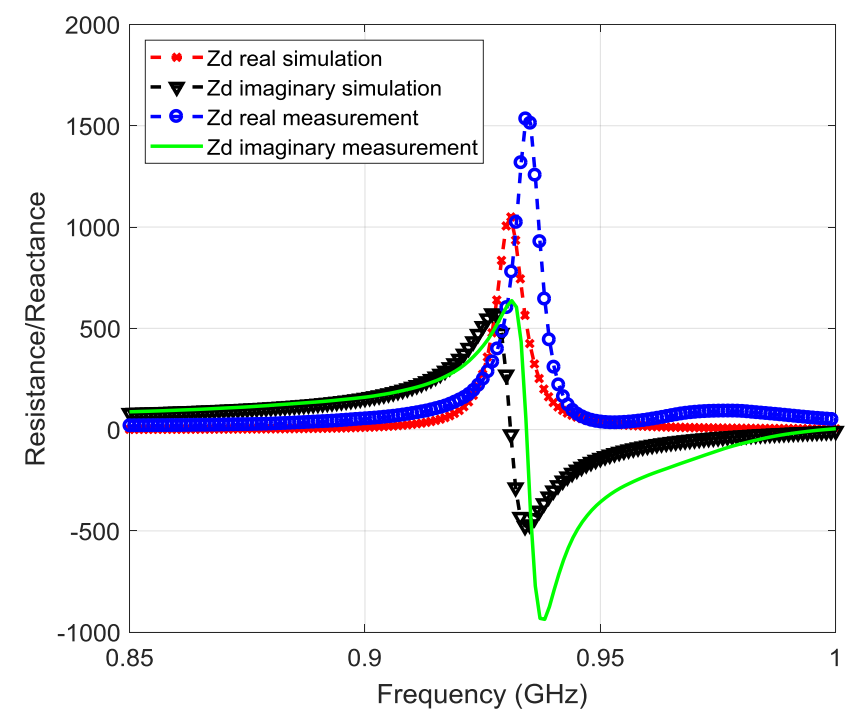

Figure 5.20. Measured and simulated input impedance of the antenna at $3.6 \mathrm{~mm}$ above the ground plane, which corresponds to an operating frequency of $902 \mathrm{MHz}$.

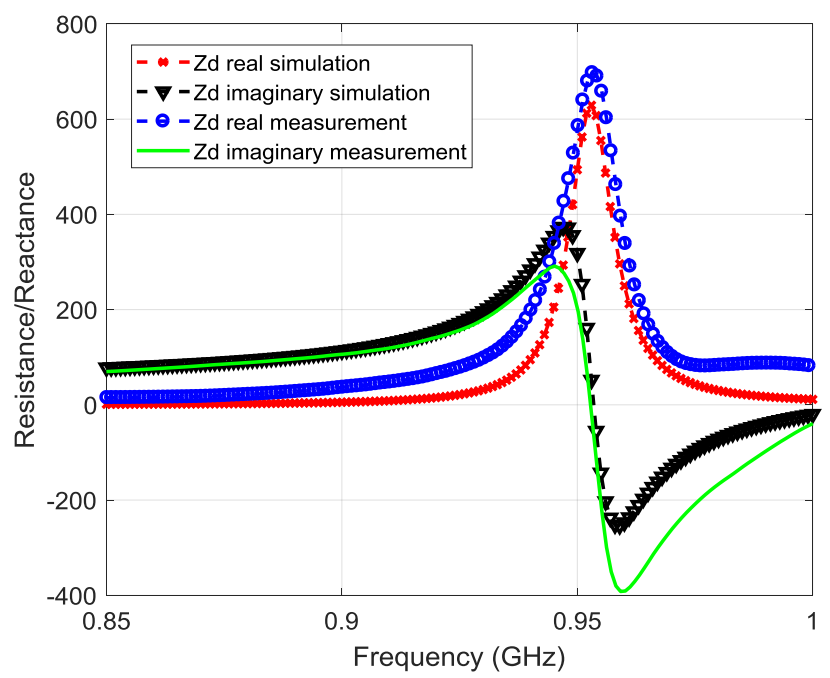

Figure 5.21. Measured and simulated input impedance of the antenna at $6.8 \mathrm{~mm}$ above the ground plane, which corresponds to an operating frequency of $928 \mathrm{MHz}$.

It is seen that the measured results agree well with the simulated results and only a small discrepancy is notable. This is attributed to the presences of the metallic test fixture near the ground plane, which induces parasitic fields that are not included in the simulation [7, pp. 340-342], [42, pp. 84-86]. 
To further understand the results depicted in Figures 5.20 and 5.21, we calculate the PTC of our antenna using (2.14). In this way, the power that the antenna delivers to the RFID IC can be quantified. The measured and simulated PTCs of our antenna are shown in Figure 5.22.

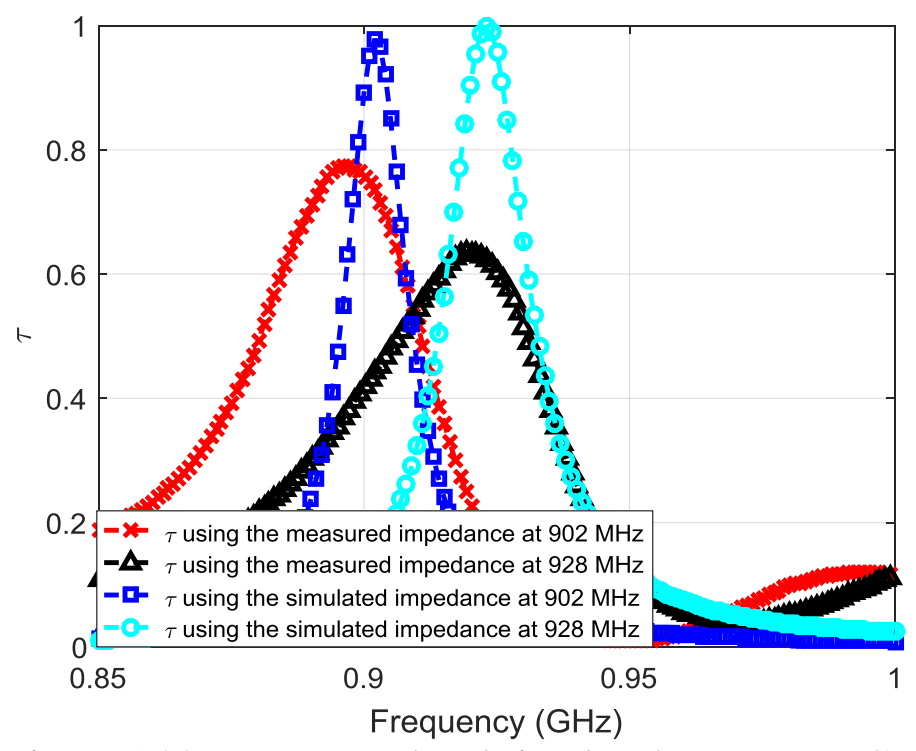

Figure 5.22. Measured and simulated antenna PTCs.

From Figure 5.22, the measured data shows that the maximum power is delivered to the RFID IC very close to the intended operating frequency of $902 \mathrm{MHz}$ and $928 \mathrm{MHz}$, which correspond to the elevation positions of $3.6 \mathrm{~mm}$ and $6.8 \mathrm{~mm}$ above the ground plane, respectively [13]. 


\subsection{Final RFID passive Temperature Sensor}

The final antenna design for the proposed temperature sensor is completed by bonding the RFID IC to the antenna using electrically conducting glue (see Figure 5.15). The final prototype is displayed in Figure 5.23.

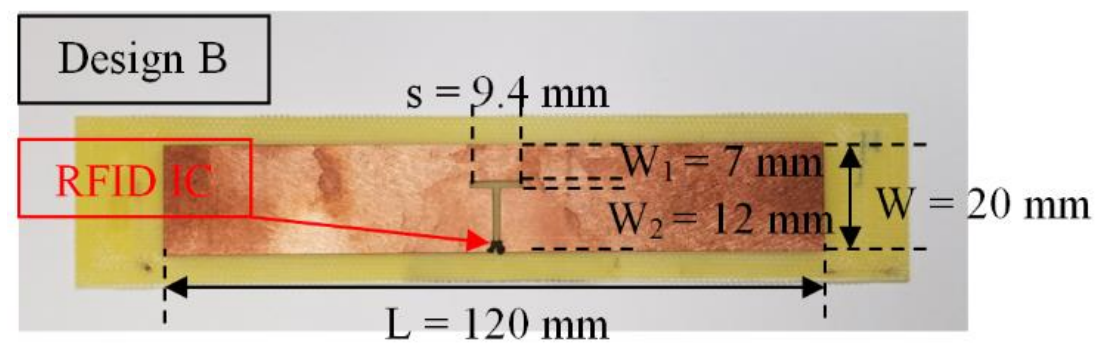

Figure 5.23. Final antenna design with RFID IC.

Our final sensor uses this antenna design, the LCE array of Figure 4.7 (which acts as a spacer that sets the antenna to the initial height of $3.6 \mathrm{~mm}$ ) and a ground plane. The sensor concept on a heating source is depicted in Figure 5.24.

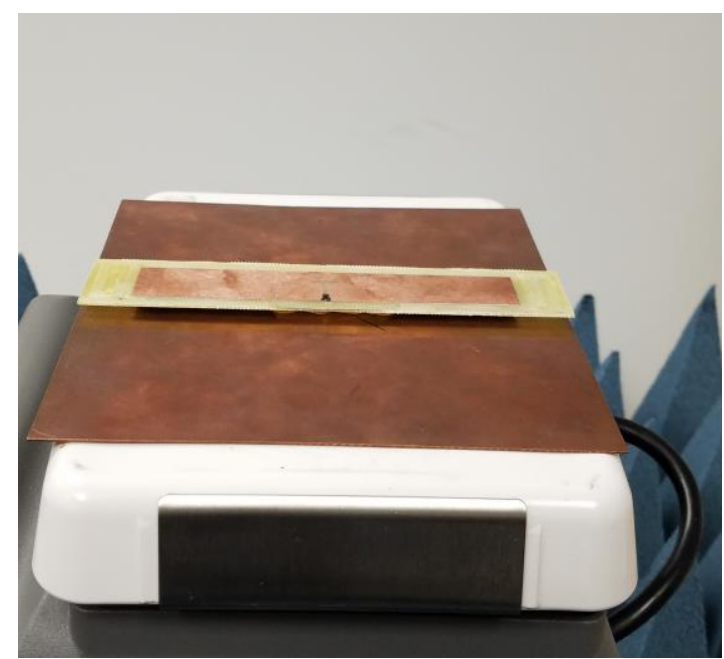

Figure 5.24. Final sensor prototype with ground plane on a heat source.

The FR4 substrate used is cost effective. Also, as discussed in Section 4.5, manufacturing the LCE array is a straight-forward process requiring materials that are easily accessible. Hence, the overall cost and the time used to fabricate this sensor is reasonable [13]. 


\subsection{RFID Measurement Setup}

Thus far, the sensor design is finalized. At this point, it is important to test the functionality of the proposed sensor in an RFID setup. Specifically, since this sensor conveys changes in temperature through controlled shifts of its operating frequency, it is essential to have a technique to measure the operating frequency of the proposed sensor. The test schematic is shown in Figure 5.25.

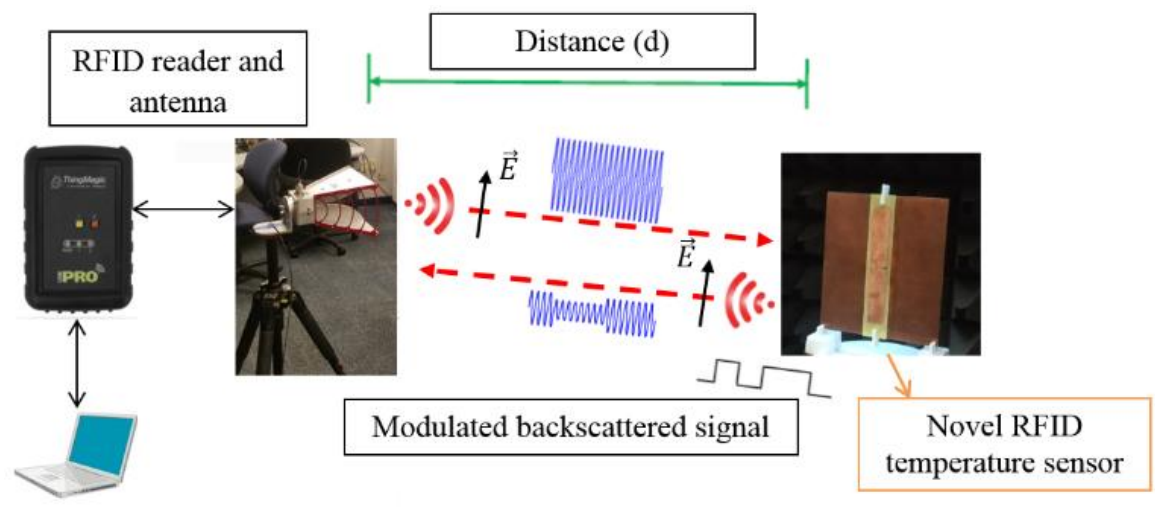

Figure 5.25. RFID measurement setup with vertical polarization orientation.

This testing arrangement consists of a computer to process data from the ThingMagic Pro RFID reader. The reader is connected to a linearly polarized double-ridge horn antenna with a gain of $7 \mathrm{dBi}$ in the RFID UHF band [77]. Moreover, the proposed sensor consists of linearly polarized planar dipole antenna. During all experiments, our sensor and horn antennas are oriented with a vertical polarization (refer to Figure 5.25) [13]. For RFID tags that are designed for situations where movement is involved, such as, the supply chain, linearly polarized tag designs are sufficient since circularly polarized reader antennas can be utilized. However, a 3dB loss due to circular-to-linear polarization mismatch will occur [7, p. 270], [13]. Furthermore, for this proposed sensor design, any polarization difference between the reader and the sensor is independent of the functionality of the sensor. 
To measure the operating frequency using the setup of Figure 5.25, the power of the RFID reader is increased in intervals of $0.1 \mathrm{dBm}$ until the threshold power of the RFID IC is reached. The threshold power is identified as the minimum power level at which data begins to be retrieved from the temperature sensor [7, pp. 70-77], [13], [35], [78]. This measured transmitted power at which the proposed sensor responds is recorded. This step is repeated for every frequency in the RFID UHF band from $902 \mathrm{MHz}$ to $928 \mathrm{MHz}$ in increments of $1 \mathrm{MHz}$. The frequency at which the least transmitted power is required to receive a response (or data) from our sensor is therefore its operating frequency [13].

To fully test the capability of the proposed design, two RFID tests are conducted. The first, which is the static test, examines the frequency shifting capability of the sensor at the calculated heights. Secondly, a dynamic test is conducted, which tests the operation of the sensor in an actual temperature varying environment.

\subsubsection{Static Test}

The first test that is conducted examines the frequency shifting capability of the sensor as the height is manually adjusted to the positions of Design B, as conveyed in Table 5.1. That is, $3.6 \mathrm{~mm}$ above the ground plane to operate at $902 \mathrm{MHz}$ and $6.8 \mathrm{~mm}$ above the ground plane to operate $928 \mathrm{MHz}$. Furthermore, supporting positioners were constructed using a 3D printer to hold the antenna steady at the corresponding heights above the ground plane. The setup for this test is shown in Figure 5.26, where the reader antenna is placed $1.143 \mathrm{~m}$ form the sensor under test. 


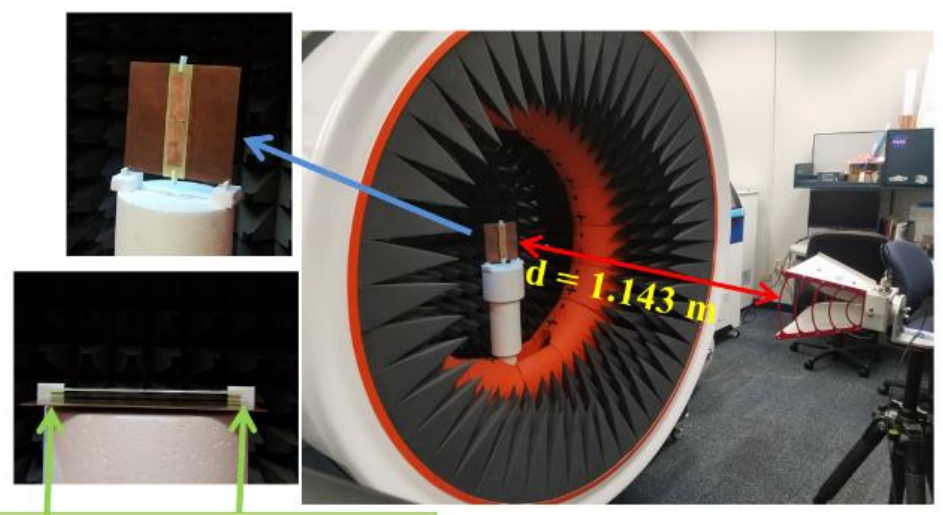

Supporting positioners for the static test

Figure 5.26. Static test setup of the proposed RFID tag.

Our measurements for the elevation position of $3.6 \mathrm{~mm}$ (which corresponds to the operating frequency of $902 \mathrm{MHz}$ ) showed the actual operating frequency slightly higher than $902 \mathrm{MHz}$. This is attributed to the presence of the 3D printed PLA positioners as they were not accounted for in the simulation. Also, the antenna was designed on an FR4 substrate that can have a slightly different permittivity from what we used in our simulations due to tolerances of the manufactured material [79]. To resolve this issue, the positioners were incrementally adjusted to allow the antenna to move closer to the ground plane (recall, the frequency and elevation height are directly proportional). It was determined that at the elevation height of $3.1 \mathrm{~mm}$, our sensor operated at the correct desired frequency of $902 \mathrm{MHz}$. Also, at the elevation height of $6.8 \mathrm{~m}$ (which was the same with the one calculated by our simulations), our sensor operated at the intended operating frequency of $928 \mathrm{MHz}$ and required no adjustment.

For parallel plate type capacitors, as the gap distance increases the capacitance decreases [7, pp. 340-342], [80]. When the gap distance is small and the capacitance is high, any change in the permittivity will result in a large change in capacitance. As a result, any adverse effects due to the presence of the positioners and/or inconsistency in the 
substrate permittivity is expected to have a larger impact to the capacitance (resulting in a larger deviation in the operating frequency) at smaller gaps. Conversely, for larger gaps, the capacitance is smaller, and the influence of the positioners and/or inconsistency to the substrate's effective permittivity has a reduced or negligible impact. Hence, a correction at the lower elevation height $(3.6 \mathrm{~mm})$ and not at the larger elevation height $(6.8 \mathrm{~mm})$ is understandable. The results of the static test of Figure 5.26 are shown in Figure 5.27.

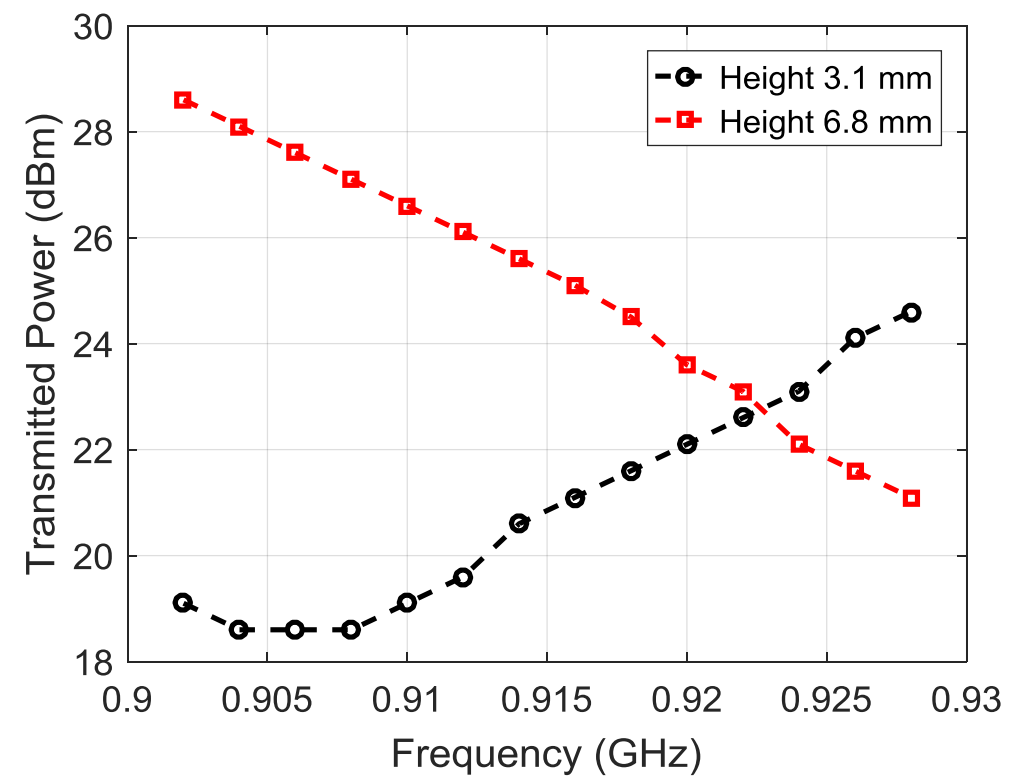

Figure 5.27. Static measurements of the RFID tag at two different heights.

These results indicate the successful operation of the designed sensor. Specifically, when the elevation position is $3.1 \mathrm{~mm}$ above the ground plane, the transmitted power is minimum at $902 \mathrm{MHz}$, which corresponds to the tag's operating frequency. Moreover, as the elevation is increased to $6.8 \mathrm{~mm}$, the operating frequency transitions to $928 \mathrm{MHz}$, where the transmitted power is least. These results agree well with our simulations. That is, according to (5.2) as the height decreases the capacitance increases. Consequently, from (5.1), as the capacitance increases the operating frequency decreases. Basically, this relation means that the height and operating frequency are directly proportional. The results 
from Figure 5.27 relay this relation successfully (i.e., at $3.1 \mathrm{~mm}$ the operating frequency at $902 \mathrm{MHz}$ and at $6.8 \mathrm{~mm}$ the operating frequency is at $928 \mathrm{MHz}$ ) [13].

\subsubsection{Dynamic Testing}

This dynamic test is conducted to verify the proposed sensor's operation under different temperature conditions. The test setup is shown in Figure 5.28, where the reader antenna is placed $0.868 \mathrm{~m}$ from the designed sensor.

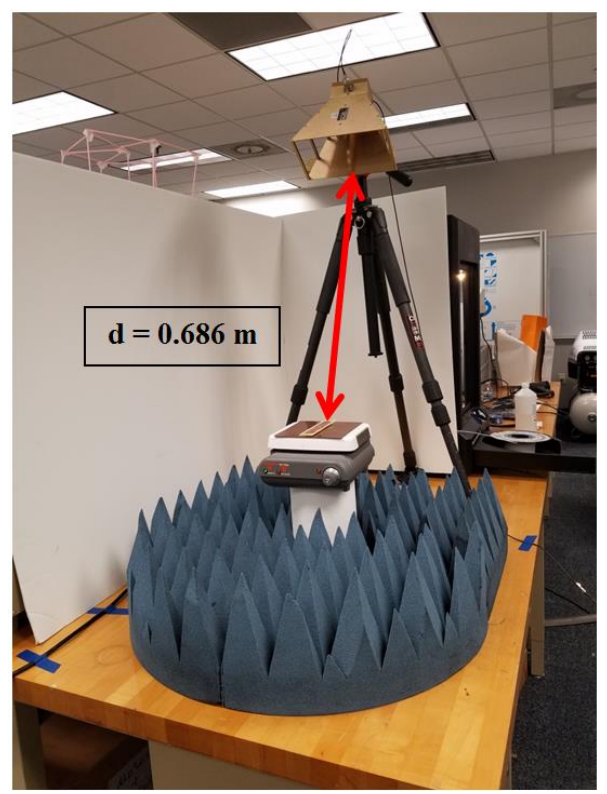

Figure 5.28. Dynamic test setup of the proposed passive RFID temperature sensor.

In this test setup, our RFID antenna is placed on top of the LCE array of Figure 4.7. The thickness of the LCE array was measured to be $1.045 \mathrm{~mm}$; thus, a spacer with a thickness of $2.055 \mathrm{~mm}$ was used to bring the elevation position of the antenna to $3.1 \mathrm{~mm}$ above the ground plane. Furthermore, the entire sensor (including the ground plane) was placed on a hot plate that served as the heat source.

As the temperature increases, the 4D printed LCE array expands from a flat state to a conical shape in the direction normal to the ground plane thereby increasing the height 
of the antenna above the ground plane (refer to Figure 4.7). The maximum actuation displacement of the LCE array was measured to be $4 \mathrm{~mm}$ [13]. Moreover, the LCE array actuates at $160^{\circ} \mathrm{C}$. Hence, at this temperature the antenna is actually raised to $7.1 \mathrm{~mm}$. The actuation of the antenna above the ground plane is shown in Figure 5.29.

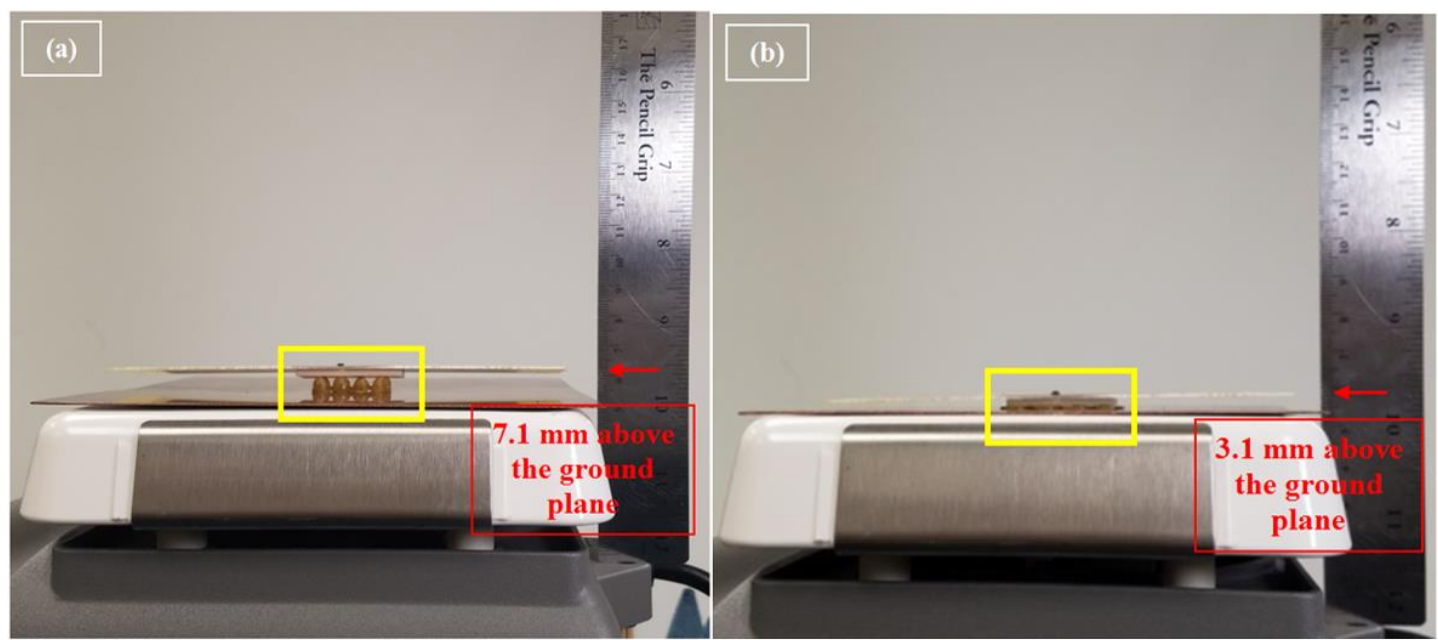

Figure 5.29. Dynamic RFID test setup with hot-plate as heat source to stimulate the LCE array. (a) The LCE actuates the antenna by $4 \mathrm{~mm}$ at $160^{\circ} \mathrm{C}$. (b) Once the heat source is turned off, the LCE automatically returns to a flat-relaxed state.

Based on our simulations, the intended elevation height of our antenna should be $6.8 \mathrm{~mm}$ above the ground plane. However, in our dynamic test, when the LCE actuates the antenna to a height of $7.1 \mathrm{~mm}$. Controlling the LCE with a higher accuracy so that we can provide an exact elevation position of $6.8 \mathrm{~mm}$ is not realistic. Also, the height of $7.1 \mathrm{~mm}$ is very close to the intended $6.8 \mathrm{~mm}$ height; therefore, it does not significantly alter the performance of our antenna at the intended operating frequency. The results from this dynamic test are depicted in Figure 5.30. 


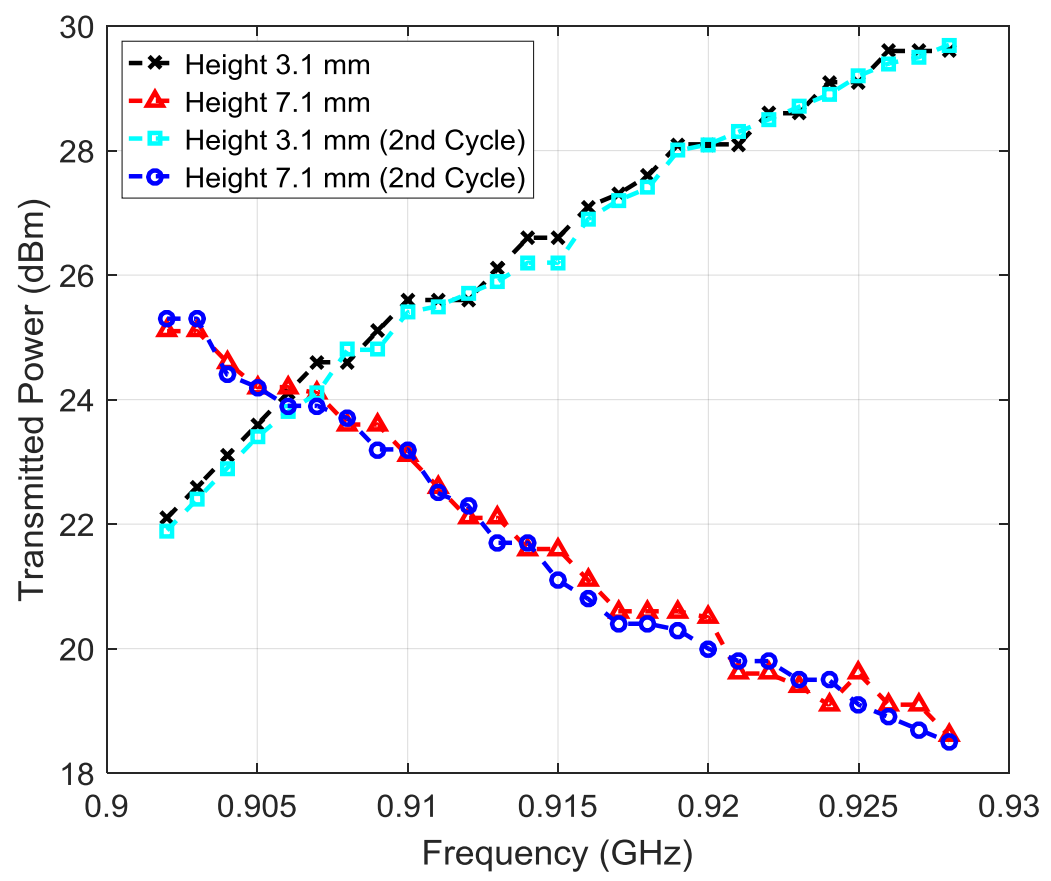

Figure 5.30. Dynamic measurements of the RFID tag at two different heights.

Figure 5.30 indicates that the proposed sensor design operates correctly. That is, at room temperature when the LCE array is at its relaxed state and the antenna is $3.1 \mathrm{~mm}$ above the ground plane, the transmitted power is the least at $902 \mathrm{MHz}$. Furthermore, when the LCE array is excited, the antenna is raised to an elevation of $7.1 \mathrm{~mm}$ above the ground plane, where transmitted power is the least at $928 \mathrm{MHz}$. Finally, to justify the repeatable nature of the developed sensor, this test was conducted again in a second cycle. That is, the sensor is removed from the heat source and the LCE array returns to its relaxed state, which takes approximately 25 seconds. Subsequently, the sensor is placed on the heat source once again. The results of the second test cycle are shown in Figure 5.30 and they compare well with the ones of the first cycle. This proves the capability of the developed sensor to measure temperature threshold violations multiple times autonomously.

As the heat source is applied, the actuation of the antenna is analog as opposed to a binary functionality. Consequently, as the antenna is actuating from $3.1 \mathrm{~mm}$ to $7.1 \mathrm{~mm}$, the 
corresponding operating frequency transitions from $902 \mathrm{MHz}$ to $928 \mathrm{MHz}$. Using the starting condition as the room-temperature (where the operating frequency is $902 \mathrm{MHz}$ ) and ending condition of $160^{\circ} \mathrm{C}$ (where the operating frequency is $928 \mathrm{MHz}$ ), a linear expression relating the temperature to the operating frequency can be formulated as follows:

$$
\text { Temperature }=5.296 \times(\text { Frequency })-4730
$$

This formulation is applicable within the frequency boundary of $902-928 \mathrm{MHz}$ and the temperature is in degrees Celsius [13].

The successful design and performance of our sensor was verified using a specialized impedance measurement technique (Section 5.5), Static RFID testing (Section 5.7.1), and Dynamic Testing (Section 5.7.2). Moreover, this sensor meets all the design objectives as outlined in Section 1.2. Primarily, this sensor: (a) utilizes purely passive technology (i.e., battery-free), (b) operates wirelessly, (c) provides a continuous form of monitoring, (d) operates autonomously without requiring resetting, and (e) has a compact form-factor to accommodate various sizes of packages. Furthermore, the physics involved in the shifting of the sensor's operating frequency is independent of the temperature (i.e., the operating frequency only depends on the actuation of the antenna above the ground plane). Also, the only component that is dependent on the temperature is the 4D printed LCE array. Hence, this design may be readily retrofitted to operate at lower temperatures using cold-reactive LCEs.

\subsection{Simulation Modeling for Practical Applications}

In the previous sections, an RFID temperature sensor using LCEs was designed and tested. However, all tests were performed in a stand-alone configuration to confirm its 
functionality. It is also important to test this sensor in practical settings. Initially, the sensor's performance is tested for cases where it is placed on a shipping box made from various materials, which carries fruits and vegetables. Then, the performance of the developed sensor is tested for cases where a second identical sensor is placed nearby with varying orientations. In this case, each sensor is placed on a separate cardboard shipping box carrying fruits and vegetables.

Fruits and vegetables are a common perishable payload in the cold-supply-chain and consist mostly of water [13], [81]. Also, shipping boxes for such perishable items are made of cardboard, wood, or plastic [82]. For this reason, we model a set of nine plastic bottles filled with fresh water that are packaged into a shipping box and vary the material properties of the shipping box accordingly. Additionally, the shipping box is modeled as a Perfect Electric Conductor (PEC) to study the effect of metallic boxes on our sensor. The simulation model of the shipping box with the fresh water bottles is shown in Figure 5.31.

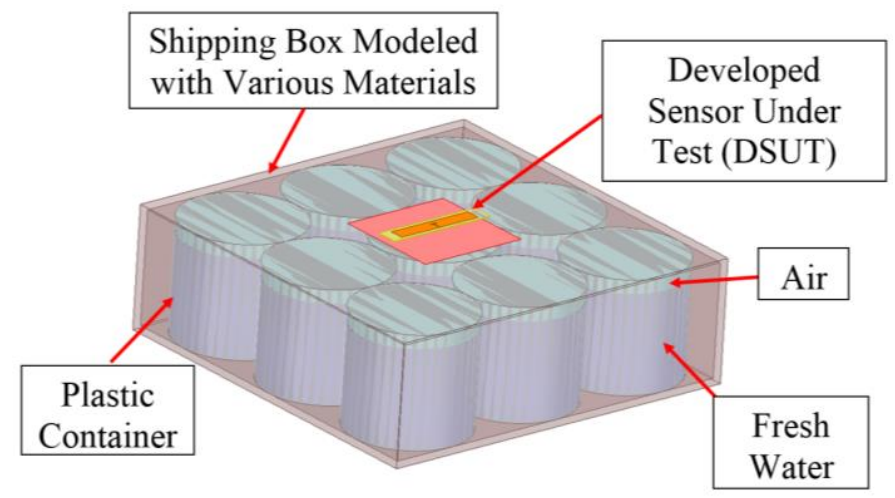

Figure 5.31. Simulation model of the shipping box modeled with various material properties and with a payload of fresh water.

The simulations examine the performance of our sensor (placed on the shipping box) for the two different elevation positions (i.e., $3.6 \mathrm{~mm}$ and $6.8 \mathrm{~mm}$ above the ground plane) that correspond to the operating frequencies of $902 \mathrm{MHz}$ and $928 \mathrm{MHz}$. This is done 
for shipping boxes that are made by various materials, namely, cardboard, wood, plastic, and PEC. The individual material properties are adjusted in the simulation by applying the corresponding relative permittivity and loss tangent that represent each material [83], [84].

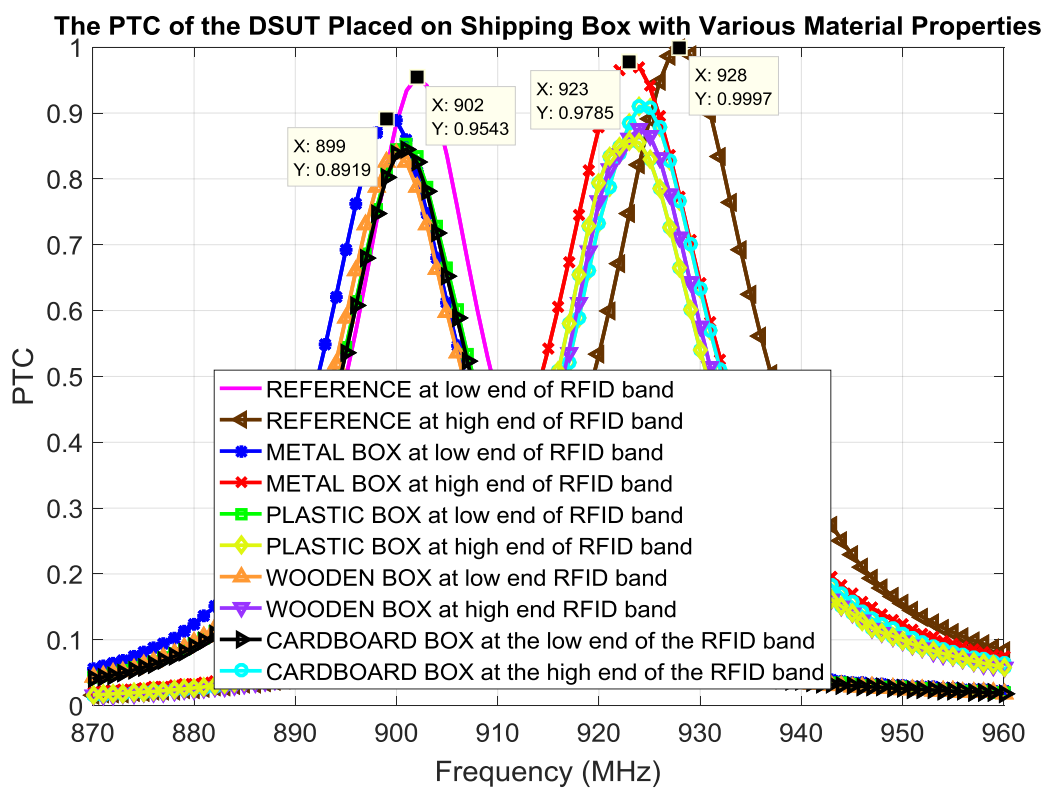

Figure 5.32. PTC simulation results of the developed sensor when placed on a shipping box made from various materials.

From Figure (5.32), the magenta and brown traces are provided as reference curves and represent the frequency response of our sensor when no shipping box is present at 902 $\mathrm{MHz}$ and $928 \mathrm{MHz}$, respectively. The remaining traces represent the frequency response of our sensor at room temperature (where the sensor operates at the lower end of the RFID band) and at elevated temperatures (where the sensor operates at the higher end of the RFID band) when mounted on top of the shipping box for different material properties of the box (cardboard, wood, plastic, and PEC). In general, all the responses (i.e., for both elevation positions) have shifted to a slightly lower frequency when compared to the reference curves. This indicates that the presence of the shipping box (for all materials) and the its payload increases the parasitic capacitance. Also, from Figure 5.32, under the presence of the 
shipping box, the PTC has decreased. This is due to the losses associated with the various materials used to make the shipping box and the contents of the payload [72, p. 456]. Nonetheless, the results from Figure 5.32 are promising.

When the antenna is at the lower elevation position and the sensor is operating at the lower end of the RFID UHF band (near $902 \mathrm{MHz}$ ), the shift in frequency is minor with the largest shift being due to the shipping box made from PEC (at $899 \mathrm{MHz}$ ). Furthermore, at $902 \mathrm{MHz}$ where the RFID UHF band begins, the response of the sensor due to all the various types of materials used for the shipping box is greater than $80 \%$; while the reference PTC, where no shipping box is present, is $95 \%$. When the antenna is at the higher elevation position and the sensor is operating at the higher-end of the RFID UHF band (near 928 $\mathrm{MHz}$ ), the largest shift in the operating frequency is due the shipping box that is modelled as PEC and the corresponding frequency is $923 \mathrm{MHz}$ while the PTC is $97 \%$. Additionally, the upper frequency responses for all the other shipping boxes have a maximum PTC larger than $85 \%$ at approximately $925 \mathrm{MHz}$. Finally, our simulation tests show that regardless of the slight decrease and shift in the PTC and operating frequency due to the presence of the shipping box and its contents, this sensor will operate successfully with good separation in the operating frequency between the two elevation positions of the antenna.

\subsubsection{Testing with Dual Sensors with Varying Orientation}

It is also important to test the functionality of the design in the presence of an additional sensor on practical items with varying orientations. Using the shipping box model (with material properties of cardboard) with the payload from Figure 5.31, a simulation test is performed where the Developed Sensor Under Test (DSUT) is placed on 
one shipping box (placed at the origin) and a second identical sensor is placed on another shipping box. The second sensor along with the shipping box is then rotated about the DSUT to three different positions of $0^{\circ}, 45^{\circ}$, or $90^{\circ}$, as shown in Figure 5.33 .

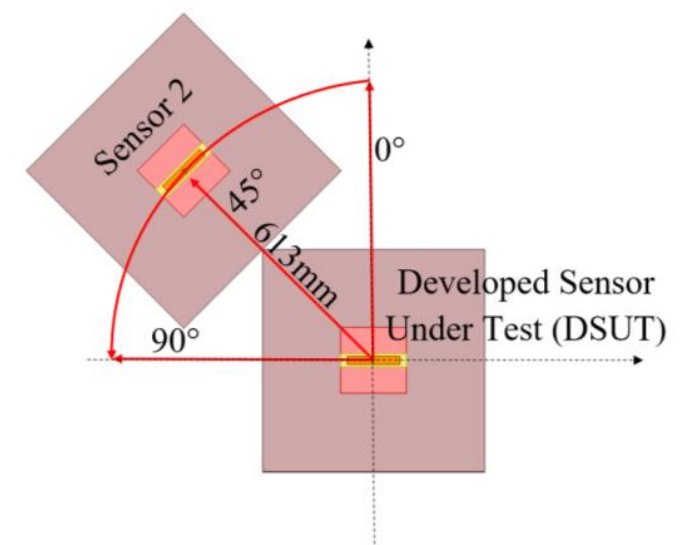

Figure 5.33. Simulation model of the DSUT in the presence of an identical sensor with varying orientations.

For each position of the second sensor and corresponding shipping box (i.e., $0^{\circ}, 45^{\circ}$, and $90^{\circ}$ in Figure 5.33), the DSUT is tested for both the low and high elevation positions (i.e., when the LCE array is in the relaxed and excited states, respectively) with the second sensor in the lower elevation state (i.e., the LCE array is in the relaxed state). Secondly, the same test is repeated; however, the second sensor is in the higher elevation state (i.e., the LCE array is in the excited state) [13]. It should be noted that when the LCE array in the relaxed state, this signifies that the ambient temperature is at room temperature and the antenna is set at its low elevation state and operates near the lower-end of the RFID UHF band (near $902 \mathrm{MHz}$ ). Also, when the LCE array is in its excited state, this indicates that the temperature has increased, and the antenna is set at its high elevation state and operates near the higher-end of the RFID UHF band (near $928 \mathrm{MHz}$ ). The results of our simulations are depicted in Figure 5.34. 


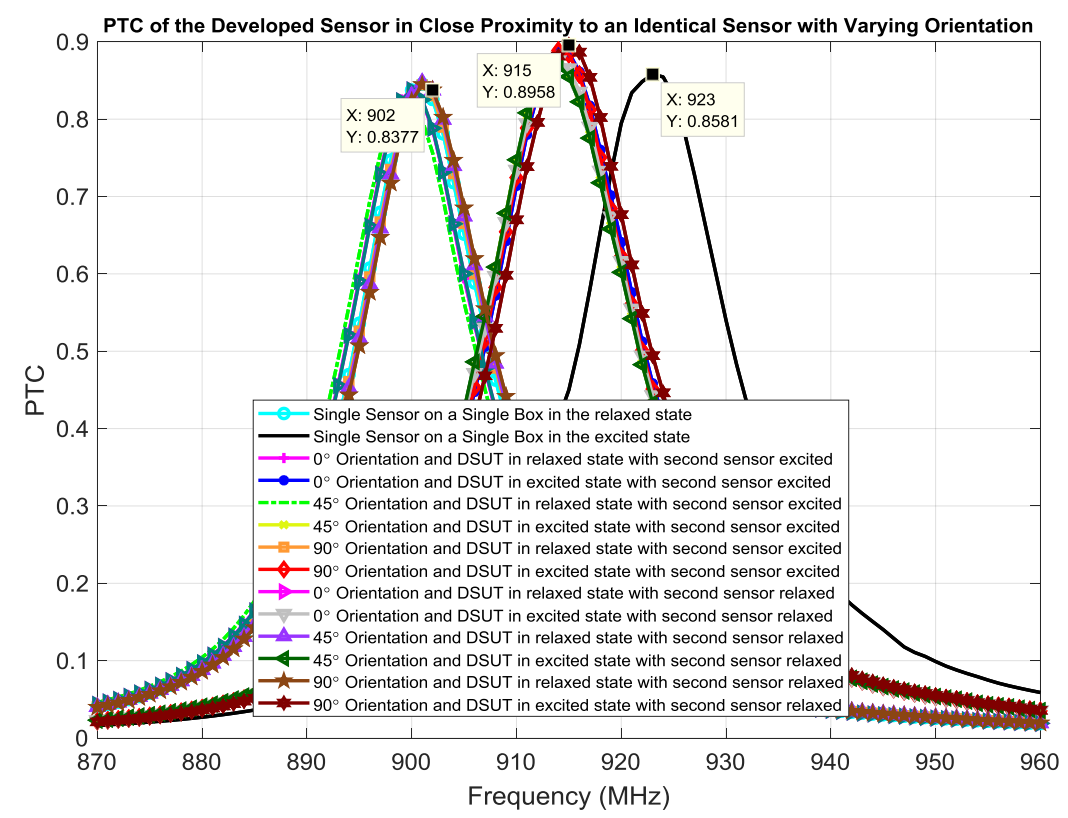

Figure 5.34. Simulated performance of our sensor at room and elevated temperatures when it placed near an identical sensor with varying.

The cyan and black traces are provided for reference and convey the performance of the DSUT in the relaxed and excited states on a single shipping box, respectively. Our results show that when the DSUT operates at room temperature with the LCE array in its relaxed state, the performance is unaffected by the presence of the second sensor and its shipping box irrespectively of their orientation. This is clearly seen in Figure 5.34 as all the curves that correspond to the operation of the DSUT in the relaxed state are almost identical to the reference curve (cyan trace). Also, the DSUT always operates sufficiently with a PTC of above $80 \%$ at $902 \mathrm{MHz}$ (which is the starting frequency of the RFID UHF band).

Moreover, according to Figure 5.34, when the DSUT operates at an elevated temperature with the LCE array in the excited state, its operating frequency shifts to 915 MHz (923 MHz was the operating frequency of a single DSUT on a shipping box when the LCE array is in the excited state as shown by the black trace in Figure 5.34). Also, regardless of the state or orientation of the second sensor (and its corresponding shipping 
box), the DSUT always operates at $915 \mathrm{MHz}$ in the presence of the second sensor in this setup. This is observed in Figure 5.34 since all the curves corresponding to the operation of the DSUT with the LCE array in the excited state have a maximum at $915 \mathrm{MHz}$ with a favorable PTC of above $85 \%$.

The shift from $923 \mathrm{MHz}$ to $915 \mathrm{MHz}$ in the operating frequency of the DSUT, when it is in the excited state, may be attributed to the inverted-parabolic relation of the plate separation to the fringing field in parallel plate capacitors [85]. Specifically, through experimentation, it is found that, as the plate separation increases, there exists a point where the fringing field is maximum as opposed to a continuously diminishing fringing field as predicted by theoretical formulas. Thus, at that critical separation, the parallel plate capacitor is more susceptible to the surroundings. That is, the fringing field lines now encounter multiple materials as opposed to just free space; hence, there now exists a larger effective permittivity as compared to exclusively the permittivity of free-space. Thus, in turn, this increases the overall capacitance from parallel plate capacitor theory as given by (5.2) [86, p. 240]. On the same note, when the DSUT is operating with the LCE array in the excited state (with the antenna actuated above the ground plane), this may be the point where the fringing fields are maximized and most susceptible to the surroundings. As a result, the presence of the additional shipping box increases the overall effective permittivity. This causes the total parasitic capacitance to increase thereby decreasing the operational frequency according to (5.1). Moreover, from the measurements in [86], at very small capacitor plate separations, the fringing field reduces and it is less susceptible to the surroundings. This is one potential explanation for the shift in the operation frequency of the DSUT from $923 \mathrm{MHz}$ to $915 \mathrm{MHz}$ in the elevated position state where the LCE array 
is excited and no shift in the operating frequency in the lower elevation state where the LCE array is relaxed (since the fringing field is less susceptible to the surroundings).

Finally, these simulated results verify the temperature detection capability of the developed sensor. Specifically, the performance is unaffected by the presence of the second sensor and the corresponding shipping box. At room temperature the sensor will be read at $902 \mathrm{MHz}$ (as this is the starting frequency of the UHF RFID band) and when the temperature reaches a certain threshold it will be read at $915 \mathrm{MHz}$. Most importantly, these two frequencies are $13 \mathrm{MHz}$ apart, which provides sufficient separation to detect that a temperature threshold has been reached [13].

\subsection{Conclusion}

In this design, a novel RFID temperature sensor is developed, analyzed and validated. The proposed sensor is fully passive; therefore, it has low-cost and long operational life. The sensor consists of a planar dipole antenna with a customized integrated T-matching network (ITMN) that is actuated above a ground plane. Consequently, the actuation causes the frequency shift.

A unique 4D-printed LCE array provides the temperature dependent actuation of the antenna above the ground plane that is reversible and repeatable. Moreover, this sensor design can be retrofitted with the appropriate LCEs to detect different ranges of temperature change. Finally, the sensor is novel and practical since it self-tunes its operating frequency in a continuous fashion as the temperature changes. This is done autonomously and without any physical intervention. 


\section{CHAPTER 6}

\section{FREQUENCY SWITCHING PATCH ANTENNA DESIGN}

\subsection{Sensor Architecture}

This sensor consists of a miniaturized patch antenna which has been customized with a slot to incorporate a switch across this slot to provide frequency switching between two chosen frequencies [87]. Typically, in antenna and RF circuit design, MEMS or PIN diodes are utilized as switches [88][89]. Although, at RF frequencies, these types of devices provide good performance when switched ON and OFF, they are active devices which require additional power sources [90] [91]. Instead, to keep the design purely passive, an ultra-subminiature mechanical switch (USMS) is used across the slot [92].

The functionality of this design can also be described by Figure 5.1. However, instead of shifting from one operational frequency to another, this design switches operational frequencies based on temperature threshold detections within the RFID UHF band. As the switch is "activated" (i.e., ON or pressed) and "deactivated" (i.e., OFF or de-pressed) the antenna switches operating frequencies between the lower and higher end of the RFID band, respectively. Also, a customized matching network is designed to match the antenna to the RFID IC at two discrete frequencies.

Furthermore, in this design, a state-of-art cold-responsive LCE array is fabricated and utilized here to reversibly actuate the switch, refer to Section 4.4. Specifically, at roomtemperature (approximately $22^{\circ} \mathrm{C}$ ), the array is in the relaxed state and the sensor operates at the lower-end of the RFID band. As the ambient temperature begins to cool to near $-10^{\circ}$ $\mathrm{C}$, the LCE array actuates and activates the switch. Consequently, the operating frequency of the sensor switches to the higher-end of the RFID band. Finally, as the surrounding 
temperature returns to room temperature the LCE array automatically returns to a relax state and the sensor switches to the original (lower) operating frequency. This process can continue through multiple temperature cycles. Hence, this sensor operates continuously and autonomously using a purely passive design.

As an important note, the RFID UHF bandwidth of $26 \mathrm{MHz}$ presents a design challenge due to its narrow range. That is, in regards to a frequency switching design as presented here, any deviation in the fabricated design (for any operating state) of approximately 4 to $5 \mathrm{MHz}$ constitutes for an unsatisfactory design. Hence, the fabrication process must be carried out very carefully.

\subsection{Patch Antenna Design with Passive Frequency Switching Capability}

Since the goal is to convey temperature threshold crossings through the frequency domain, an antenna that operates with a narrow bandwidth is required. Conversely, if an antenna with a large bandwidth is utilized, no difference in the operating frequency would be conveyed as the two operating states would overlap and would be indistinguishable from each other, refer to Figure 5.1. Therefore, for its inherently narrow bandwidth of operation, the patch antenna is used in this sensor design [34, p. 67]. Furthermore, the USMS is originally designed for DC applications and it provides only an approximate short and open at its activated and deactivated modes, respectively. For this reason, the full RF characterization of the USMS is conducted and incorporated into the design using both ANSYS HFSS and Circuit Designer in a co-simulation technique to obtain the correct antenna and integrated-slot combination. The antenna is designed using a ceramic polymer composite substrate (i.e., Rogers TMM 13i) with a high dielectric constant (i.e., $\varepsilon_{r}=12.85$ ) 
[93]. The high dielectric constant of this substrate compresses the operational bandwidth of our antenna thereby improving the frequency selectivity of the sensor [94].

\subsubsection{Patch Antenna Theory}

The patch antenna is a dielectric-loaded cavity of length, $L$, and width, $W$, bounded by electric conductors on the top and bottom, which serve as the metallic patch and groundplane, respectively, refer to Figure 6.1 [95, pp. 90-96] [96].

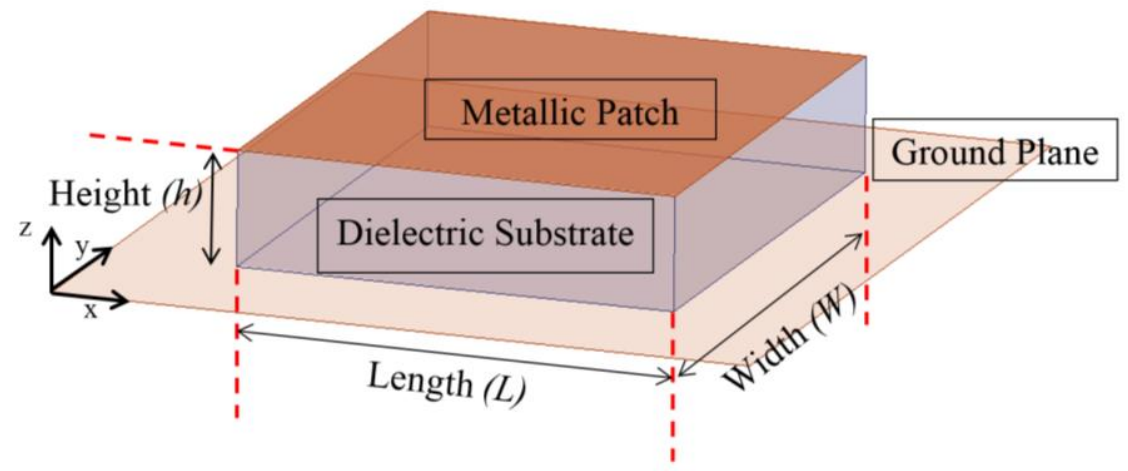

Figure 6.1. Patch antenna model.

The electric conductors serve as electric-walls (where the tangential electric fields vanish) and the perimeter serve as magnetic-walls (where the tangential magnetic fields vanish). Moreover, the metallic patch is very close to the ground plane $\left(h \ll \lambda_{g}\right)$, where $\lambda_{g}$ is the guided wavelength within the medium. Consequently, there is no field variation along the z-axis. Hence, the electric field is virtually normal to the metallic patch. As a result, the cavity supports a $T M^{Z}$ field profile [33, p. 266]. Moreover, the dominant mode of the patch antenna occurs when $L>W>h$ and is designated as the $T M_{10}^{Z}$ mode [33, p. 830]. The corresponding field profile is depicted in Figure 6.2. 


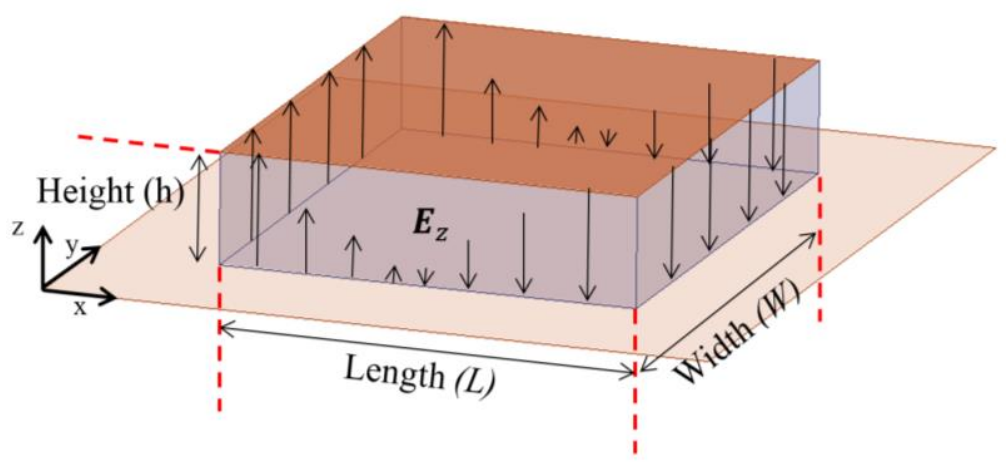

Figure 6.2. The dominant mode of the patch antenna has a $\mathrm{TM}_{10}^{\mathrm{z}}$ field profile.

The resonant frequency, $f_{r}$, of the patch antenna (for the $T M_{10}^{z}$ mode) is dependent on the electrical length, $L$, and is approximated by [95, p. 266]:

$$
\mathrm{L} \approx \frac{\lambda_{\mathrm{g}}}{2}=\frac{\mathrm{c}}{2 \mathrm{f}_{\mathrm{r}} \sqrt{\varepsilon_{\mathrm{r}}}}
$$

where $\varepsilon_{\mathrm{r}}$ is the relative permittivity of the substrate. For this mode, the electric and magnetic field profiles are expressed with the following relations [33, p. 833]:

$$
\begin{aligned}
& \mathrm{E}_{\mathrm{z}}=\mathrm{E}_{0} \cos \left(\frac{\pi}{\mathrm{L}} \mathrm{x}^{\prime}\right) \\
& \mathrm{H}_{\mathrm{y}}=\mathrm{H}_{0} \sin \left(\frac{\pi}{\mathrm{L}} \mathrm{x}^{\prime}\right)
\end{aligned}
$$

where $x^{\prime}$ represents a point along the $x$-axis. Accordingly, from (6.2), the electrical field is maximum at the ends of dielectric-cavity (however, they are inverted with a $180^{\circ}$ phase reversal) and theoretically zero at the center. Thus, this is a half-wavelength rectangular patch, where the length is equal to half the guided wavelength, refer to Figure 6.2. Additionally, from (6.3) the tangential magnetic field within the dielectric-cavity is minimum at the ends and maximum at the center. This tangential magnetic field induces a 
surface current distribution on the metallic patch $J_{S}$, which can be modelled as a perfect electric conductor. This surface current is due to the following boundary condition [33, $\mathrm{p}$. 18][96]:

$$
\overrightarrow{\mathrm{J}_{\mathrm{s}}}=\hat{\mathrm{n}} \times \overrightarrow{\mathrm{H}}
$$

where $\hat{n}$ is a unit vector normal to the surface of the metallic patch. From (6.3) and (6.4), the surface current distribution is maximum in the center, theoretically zero at the ends, and flows along the length of the patch. As an example, this current distribution (for the $T M_{100}^{Z}$ mode) is illustrated in Figure 6.3.

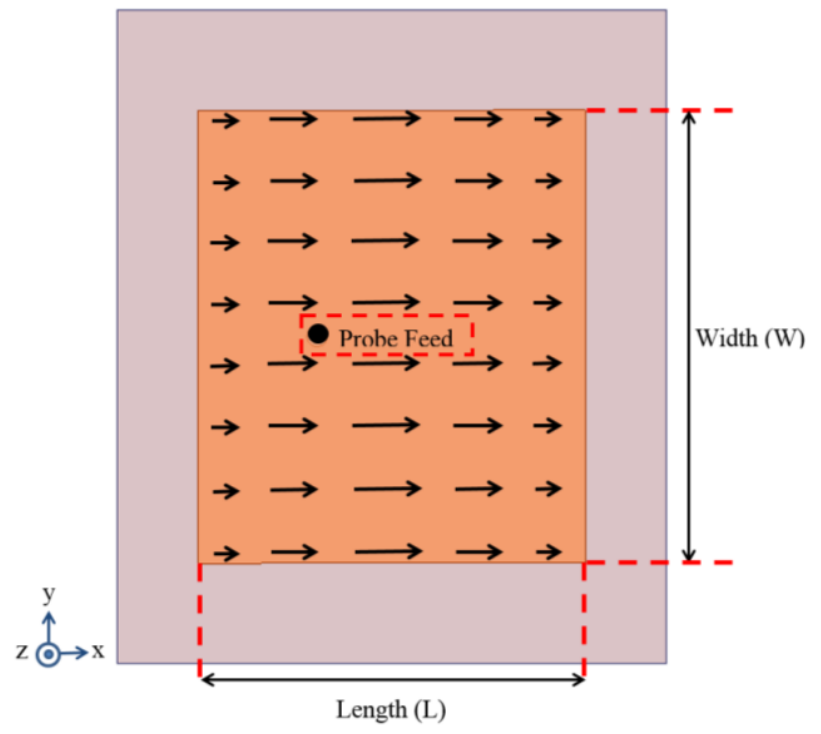

Figure 6.3. Surface current distribution for the $\mathrm{TM}_{10}^{\mathrm{Z}}$ mode. 
In summary, the magnitude of the voltage, current, and impedance distribution along the length of the metallic patch, operating with the $T M_{10}^{Z}$ mode, may be summarized by Figure 6.4 .

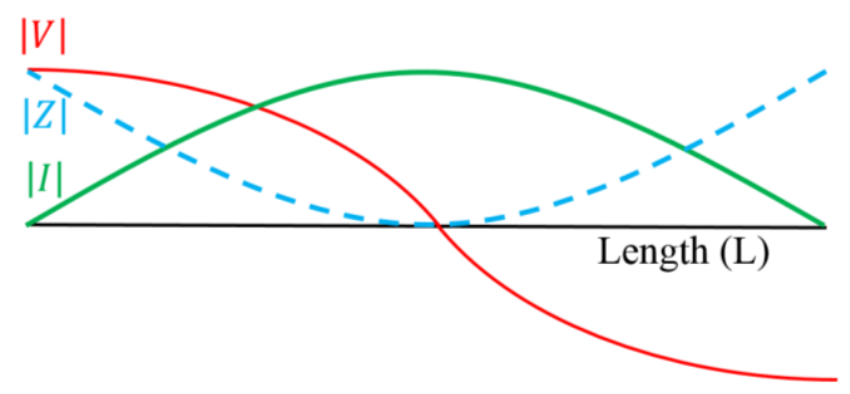

Figure 6.4. The voltage, current, and impedance profile over the length of the patch for the dominant mode.

Finally, the antenna in this sensor design will incorporate a probe feed. This type of feed promotes a design with minimized area. Also, to further keep the area small, the matching network is designed separately and connected to the antenna in a vertical formation as it will be discussed in a subsequent section.

\subsubsection{Integrated Slot Design for Frequency Switching}

A patch antenna may be integrated with a slot in such a way that when a short is applied across the slot, a shift between two desired operating frequencies is achieved [88]. Furthermore, this technique for achieving frequency switching from a patch antenna serves only as a concept herein. This is because, in this research, a short-circuit is not applied to the slot but rather the passive electrical load of the USMS is applied. The modification involves incorporating the measured full RF characterization of the USMS in a cosimulation technique, which is detailed in the following sections. Nonetheless, the original concept is critical as it serves as the foundation to the frequency switching capability. 
According to [88], the electrical length, $L$, of the metallic patch can be extended by introducing slots on the surface of the metallic patch. As a result, this reduces the resonant frequency of the patch as governed by [33, p. 266];

$$
\mathrm{f}=\frac{\mathrm{c}}{2 \mathrm{~L} \sqrt{\varepsilon_{\text {eff }}}}
$$

where $\left(\varepsilon_{e f f}\right)$ is the effective permittivity. The introduction of the slot effectively increases the electrical length, $L$, because the surface currents on the metallic patch have a longer path to travel. This notion is depicted in Figure 6.5, where the modified electrical length, $L^{\prime}$, of the patch is larger than the initial electrical length, $L$. Consequently, from (6.5), as the slot is integrated the resonant frequency is reduced since the effective electrical length has increased (from $L$ to $L^{\prime}$ in Figure 6.5).
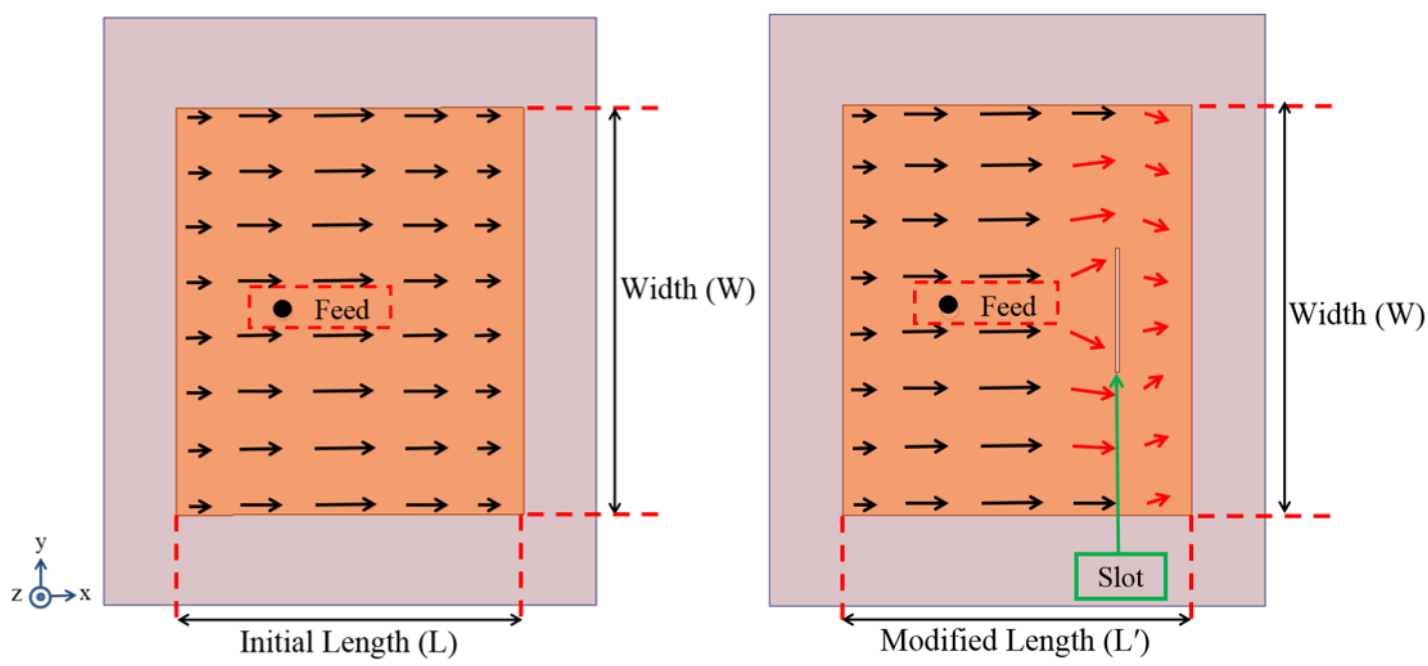

Figure 6.5. When a slot is added to a metallic patch, the current has a longer path to travel which increases the length of the patch (from $L$ to $L^{\prime}$ ) and decreases the resonant frequency as per (6.5).

Furthermore, by applying a short-circuit across the slot, the modified effective electrical length of the patch is once again reduced as the short-circuit provides a path for the current to flow. As a result, the resonant frequency is once again increased. However, the applied 
short does not increase the frequency to the initial operating state where no slot was present. For this reason, the starting point of designing such an antenna is to begin with a basic patch antenna that resonates at a frequency slightly higher than the RFID UHF band, for example at $930 \mathrm{MHz}$. Through this technique, controlled switching of the operating frequency is achieved. As an example, the performance trends discussed above are verified by the simulated $\mathrm{S}_{11}$ (see Figure 6.5) of an RFID patch antenna. Specifically, a patch antenna was designed to operate at $930 \mathrm{MHz}$ (red curve), a slot with certain dimensions was then found to retune the antenna to operate at $905 \mathrm{MHz}$ (blue curve), after a short is applied the antenna operates at $920 \mathrm{MHz}$ (cyan curve). Hence, by adding or removing a short, the antenna switches its operation between $920 \mathrm{MHz}$ and $905 \mathrm{MHz}$ within the RFID UHF band, respectively.

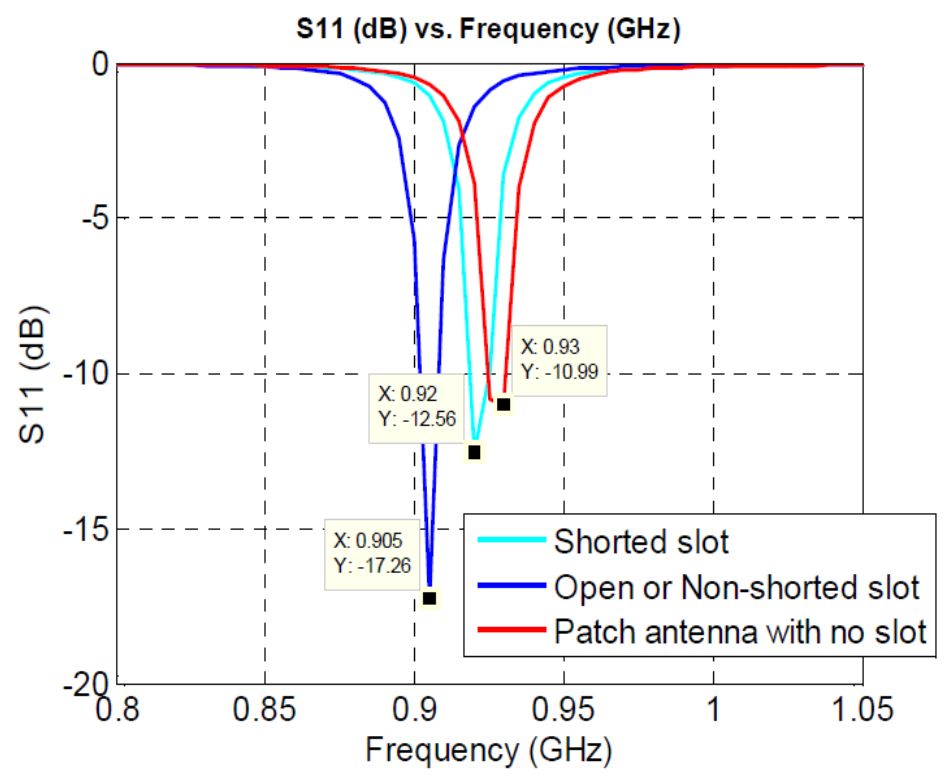

Figure 6.6. Simulated $S_{11}$ of a patch antenna with and without a slot.

It is important to note that the dimension of the slot must be calculated carefully to provide the proper frequency switching within the desired frequency band. In this research, this was done through a parametric sweep using ANSYS HFSS. First, a patch is designed 
at the desired frequency $(930 \mathrm{MHz})$. Secondly, a slot is added along with a parametric sweep to find the desired low-frequency operating point within the RFID UHF band (i.e., $902 \mathrm{MHz}$ ). Finally, this slot dimension must also support the switching of the operating frequency, through an applied short across the slot, to the desired increased frequency operating point within the RFID UHF band. This notion is conveyed in the simulated performance of Figure 6.6.

\subsubsection{Passive Sensor Design with the USMS}

In this research, the above-mentioned concept is applied with a modification. That is, instead of applying a short, the antenna's frequency of operation is switched by a mechanical switch, which is placed across the patch's slot, refer to Figure 6.7. Specifically, a USMS made by Omron Electronics, which has a single-pole double-throw (SPDT) functionality, is used [92]. This switch will permit the continuous and independent operation of the sensor without the use of any external voltage sources, which would be required by MEMS or diode switches (i.e., this sensor is battery-free). Instead, the switch will be activated by the cold-responsive LCE array, which operates reversibly. In other words, the USMS automatically returns to the original position once the LCE relaxes. 


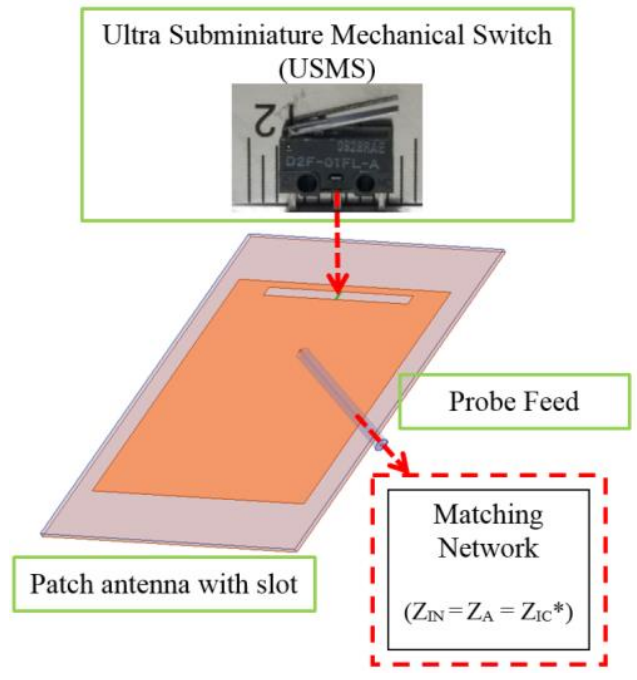

Figure 6.7. The USMS is connected across the slot and provides the passive frequency switching capability of the antenna when it is activated and deactivated.

\subsubsection{Co-Simulation Setup and Antenna Design}

MEMS and PIN diode switches may be simulated in HFSS by the typical application (or not) of a PEC to model the effect of the switches in the activated and deactivated states. For this case, a single full-wave solver, such as, ANSYS HFSS is sufficient for modeling. However, as mentioned earlier, these boundary conditions will not suffice in modelling the USMS. For this reason, the complete electrical characterization of the USMS must be incorporated into the simulation model. This is accomplished by measuring and incorporating the full two-port scattering parameter measurements of the USMS (at the RFID UHF band) in the simulation [40, p. 178]. We utilize ANSYS Circuit Designer to merge the EM simulation conducted by HFSS and the physical measurements of the USMS in a co-simulation technique [97], [98].

As a first step, we convert the patch antenna design into a two-port network in ANSYS HFSS. That is, an additional port is placed across the slot, in the same location where we intend to place the USMS. Intuitively, this establishes an electrical-connection 
between the feed port (port 1) and the newly added "slot" port (port 2). Subsequently, exporting the corresponding s2p file for this antenna model into ANSYS Circuit Designer, one can then add various electronic elements to the "slot" port and study the effect at the feed port. In the case of this design, the USMS will be applied to the slot port as depicted in Figure 6.8.

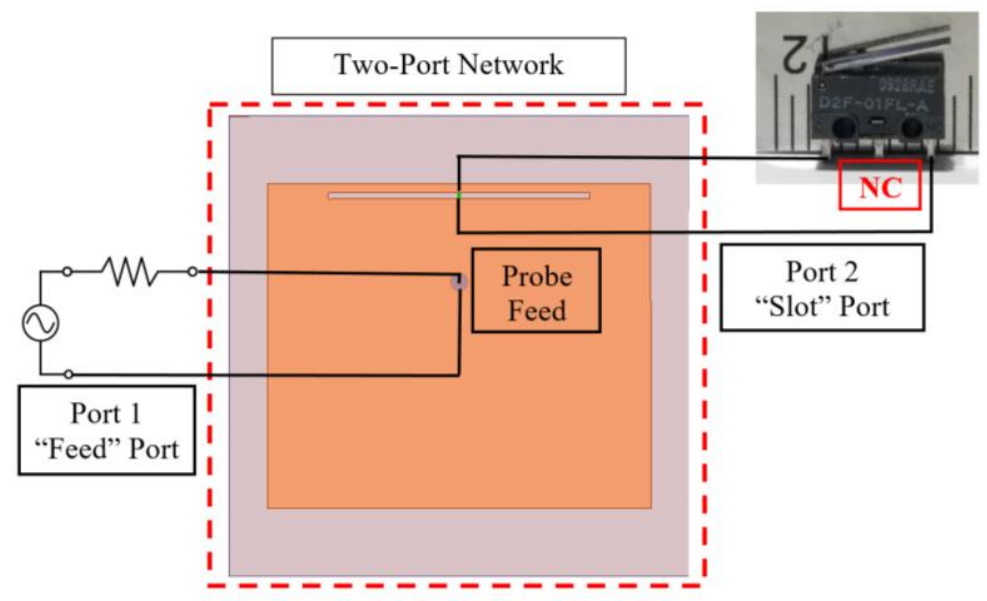

Figure 6.8. The simulated patch antenna is converted to a two-port system by adding an additional port at the slot, where we intend to connect the switch.

Ultimately, the goal is to find the dimensions of the slot so that when the USMS is applied, the desired frequency switching (within the RFID UHF band) occurs at the feed port when the switch is activated and deactivated. To accomplish this, a parametric sweep of the dimensions of the slot in terms of the length, width, and position is conducted (see Figure 6.9) and each iteration is exported into Circuit Designer. 


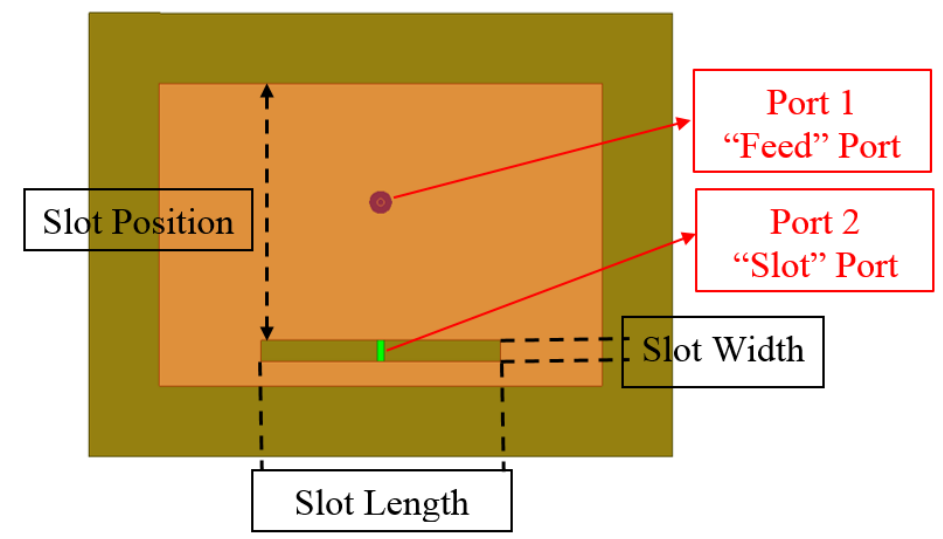

Figure 6.9. A parametric sweep of the slot width, length, and position is conducted using HFSS.

At this point, the full electrical characterization of the USMS is conducted by taking the two-port S-parameters within the RFID UHF bandwidth (i.e., $902 \mathrm{MHz}$ to $928 \mathrm{MHz}$ ) for when the switch is activated and deactivated. This was done by first fabricating a microstrip test fixture to connect to the terminals of the USMS. This test fixture is shown in Figure 6.10.

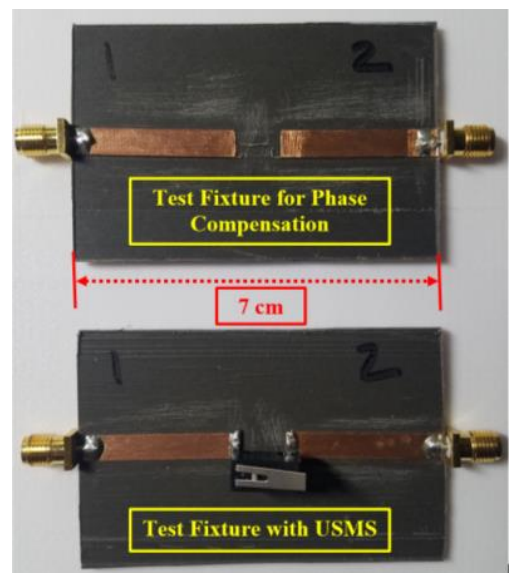

Figure 6.10. Microstrip test fixture for connecting and measuring the switch. (Top) Fixture without switch for phase compensation. (Bottom) Fixture with switch. Initially, the test fixture without the switch is used to compensate for the electrical length by using the auto-port correction feature in the E5061B Vector Network Analyzer. Then, the switch is connected to the fixture and the 2-port scattering parameters are taken for both ON and OFF states of the switch. The measured results are shown in Figure 6.11. 


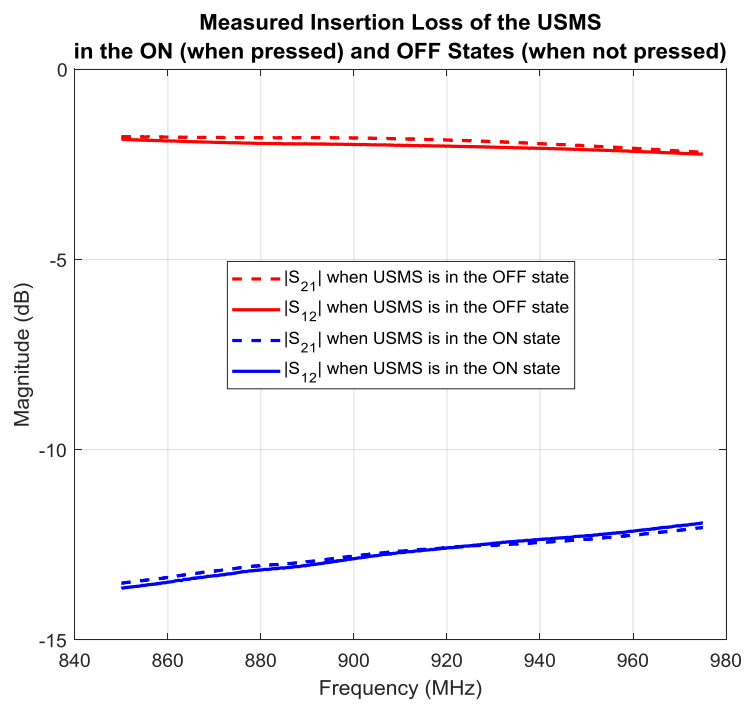

Figure 6.11. Measured insertion loss of the switch at its ON and OFF states.

The measurements show that in the ON (i.e., pressed or activated) and OFF (i.e., de-pressed or deactivated) states, the insertion loss is approximately $12.5 \mathrm{~dB}$ and $2 \mathrm{~dB}$ within the RFID UHF band, respectively. In comparison, typical MEMS provide an insertion in the $\mathrm{ON}$ and OFF states of approximately $0.45 \mathrm{~dB}$ and $25 \mathrm{~dB}$, respectively [90]. Also, PIN diodes have an insertion loss in the $\mathrm{ON}$ and OFF states of approximately $1 \mathrm{~dB}$ and $60 \mathrm{~dB}$, respectively [91]. Therefore, as mentioned in Section 6.2, the USMS provides only an approximate short and open in the ON and OFF states, respectively, and requires a co-simulation technique that will be discussed here.

The $s 2 p$ files corresponding to the switch measurements are imported to Circuit Designer and connected to the "Slot" port (see Figure 6.9) of the antenna model, which consists of simulation data for numerous slot dimensions (as a dimensional sweep for the length, width, and position of the slot was conducted in ANSYS HFSS). This model setup is shown in Figure 6.12 below. 


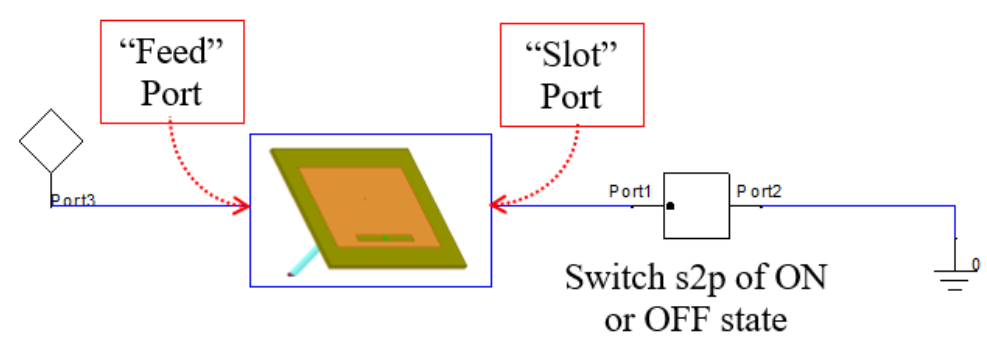

Figure 6.12. ANSYS Circuit Designer setup for connecting the measured switch data to the antenna.

From the model in Figure 6.12, the switch can now be turned $\mathrm{ON}$ and OFF, and the frequency response is examined at the feed port. As an example, the relationship of the slot position to the operating frequency for various widths and lengths of the slot with the switch applied in the ON of OFF state is depicted in Figures 6.13 and 6.14, respectively.

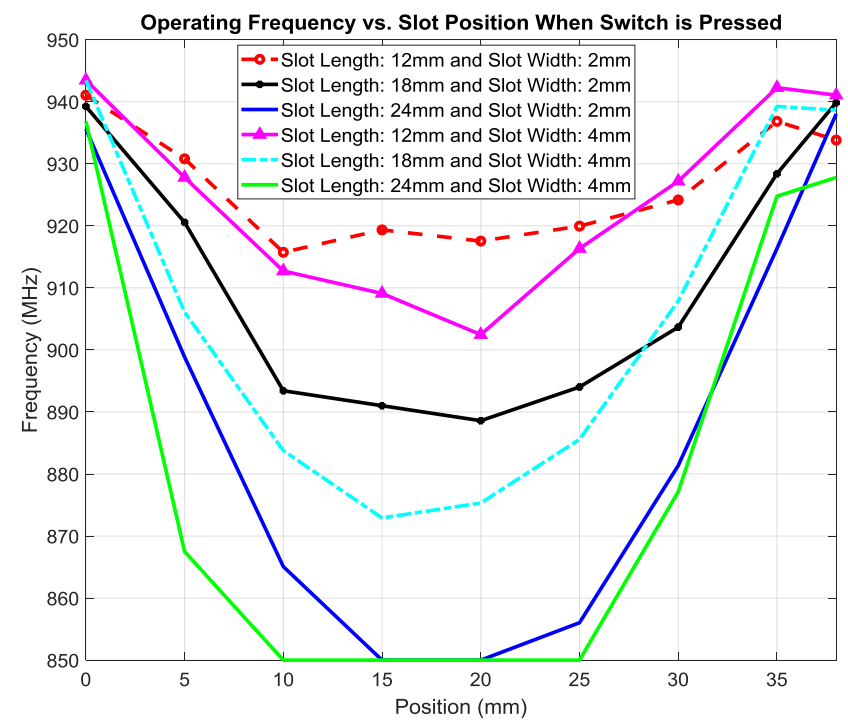

Figure 6.13. Effect of the operating frequency of the proposed antenna with the switch activated as a function of slot position, length, and width. 


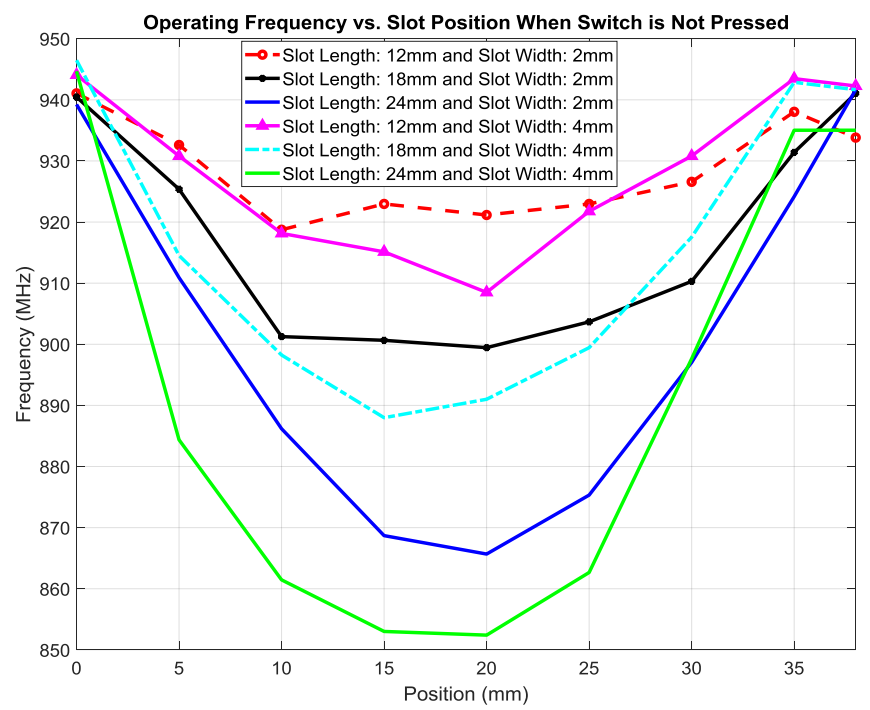

Figure 6.14. Effect of the operating frequency of the proposed antenna with the switch deactivated as a function of slot position, length, and width.

Essentially, the goal is to find the largest frequency switching bandwidth (i.e., the difference of the antenna's operational frequencies at the ON and OFF states of the switch). This bandwidth should be as large as possible but should not exceed the RFID UHF bandwidth of $26 \mathrm{MHz}$. Also, this frequency switching bandwidth should be within the band of $902 \mathrm{MHz}$ to $928 \mathrm{MHz}$. Unfortunately, no design meets these two criteria exactly. Consequently, the design that closely meets these criteria is selected. The final design has the following dimensions: Slot Length: $34 \mathrm{~mm}$, Slot Position: $36.5 \mathrm{~mm}$, and Slot Width: $3 \mathrm{~mm}$ (see Figure 6.9). However, to correct any deviation in the performance from the above mentioned criteria, a customized two-stage matching network is designed. The first stage of the matching network shifts the operating frequencies of the antenna to within the RFID band (i.e., 902-928 MHz). Moreover, the second stage of the matching network matches the $50 \Omega$ response at the two operating frequencies to the conjugate input impedance of the RFID IC. The design of this matching network is further detailed in a subsequent section. For the selected design, the simulated response is shown in Figure 6.15. 


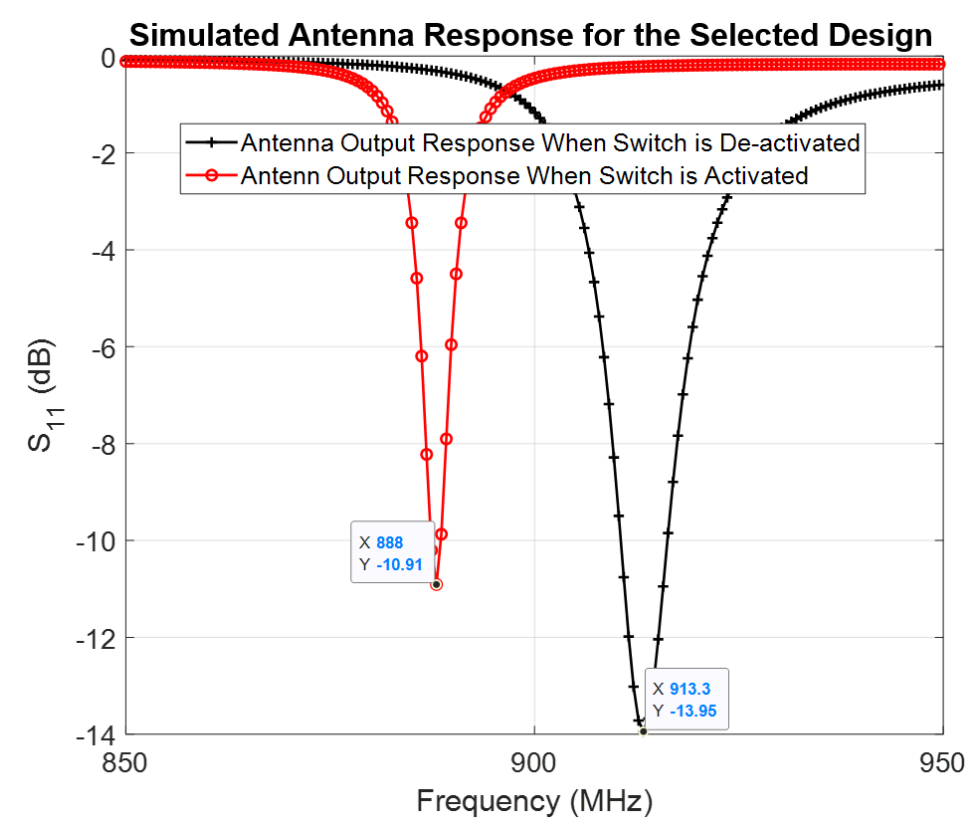

Figure 6.15. Simulated $S_{11}$ for the selected antenna design.

The operational frequency of the selected antenna is $913.3 \mathrm{MHz}$ and $888 \mathrm{MHz}$ at the OFF and ON switch states, respectively. Also, there is a $25.3 \mathrm{MHz}$ difference between the two frequencies of operation (i.e., switching bandwidth). This switching bandwidth is relatively large (compared to the RFID UHF bandwidth of $26 \mathrm{MHz}$ ) thereby providing a clear distinction between the two states (i.e., one can reliably determine which state the antenna is operating at based on the operational frequency). From Figure 6.15, it is apparent that the response of the selected design for when the switch is activated (or in the ON state) lies outside the RFID UHF band. This is contradictory to the criteria mentioned earlier. However, this problem will be addressed by the first stage of the matching network. Nonetheless, at this point, it is important to note that an antenna design has been derived that operates at two desired frequencies, which are controlled by the USMS. 


\subsection{Integration of Antenna and LCE Actuator}

The patch antenna design with the selected dimensions was fabricated using Rogers TMM 13i substrate, which has a dielectric constant of 12.85. Subsequently, the switch was soldered across the slot. The prototype antenna is shown in Figure 6.16.

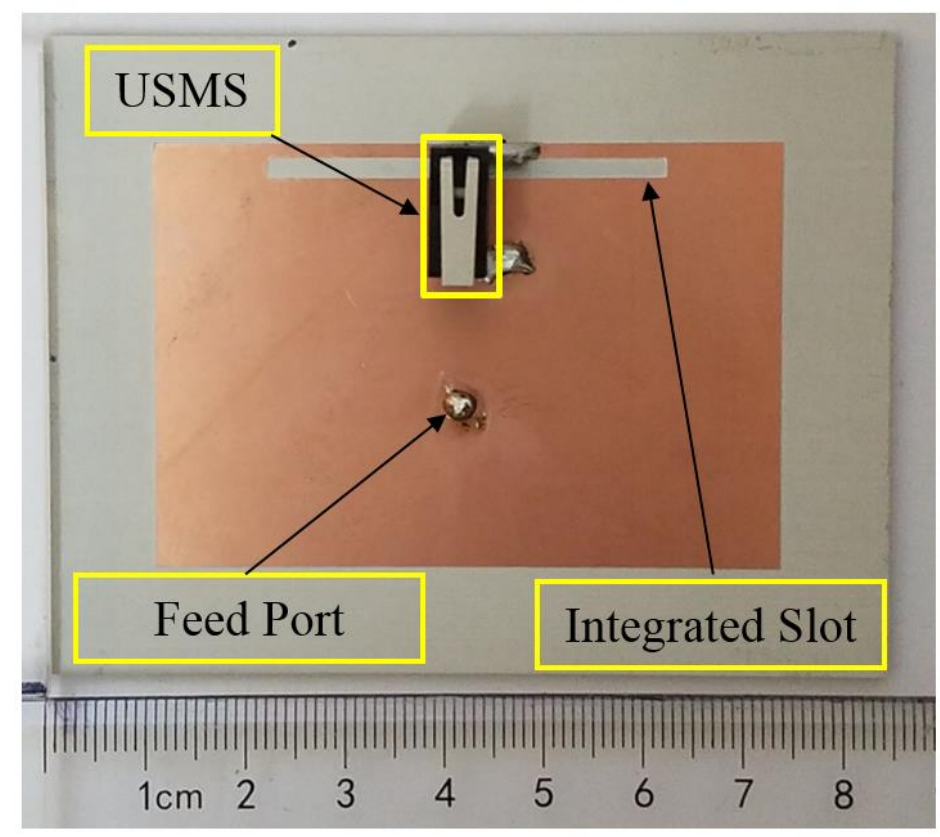

Figure 6.16. Fabricated antenna with the switch soldered across its slot.

At this point, an effective way for applying the longitudinal mechanical force of the LCE triangular array (see Figure 4.9) to activate the USMS is essential. This is accomplished by designing and fabricating a 3D printed housing for the LCE array as shown in Figure 6.17. 


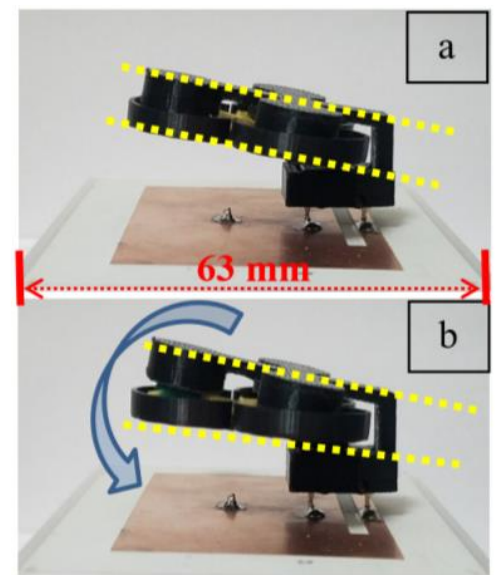

Figure 6.17. 3D printed frame that translates the actuation of the LCE array. a) The LCE is at its relaxed state and the switch is set at its OFF (de-pressed or deactivated) state. (b) The LCE is actuated and the switch is set to its ON (pressed or activated) state.

This housing includes two components. The first, is a housing that is fixed to the base of the USMS and provides a static reference for the LCE array to apply a force. The second, is a platform that is placed on the spring-loaded dynamic lever of the USMS. Finally, it is important to recall that (refer to Section 4.4) the most current technology in cold-responsive LCEs provide small displacements during excitation. In this case, a maximum displacement of $1.23 \mathrm{~mm}$ at approximately $-9^{\circ} \mathrm{C}$ is achieved. Therefore, the LCE platform in Figure 6.17 must be designed and adjusted until the lever of the USMS can be successfully triggered within the displacement range of the LCE array.

The antenna was measured using the Agilent E5071C ENA at the two different states of the switch. This measurement is conducted to validate the accuracy of the design process, i.e., the co-simulation technique. The test setup is shown in Figure 6.18. 


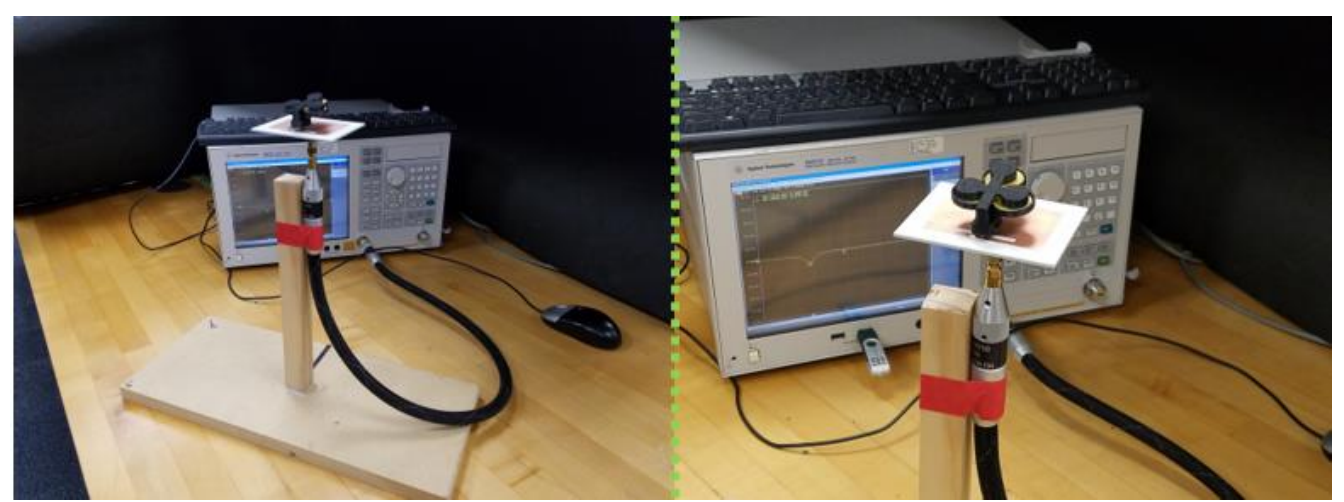

Figure 6.18. VNA test setup and fabricated antenna.

The measured rand the simulated results are compared displayed in Figure 6.19. The measured results agree well with the simulated results in terms of the frequency of operation and the magnitude of the reflection coefficient. Moreover, the measured frequency switching bandwidth of the fabricated design agrees well with the simulated design (for both switch states). That is, the proposed design provides a bandwidth of 24.7 $\mathrm{MHz}$ and the ideal simulated response has a bandwidth of $25.3 \mathrm{MHz}$. These bandwidth values are desired as they are slightly less than the RFID UHF bandwidth of $26 \mathrm{MHz}$. This ensures that the maximum PTC will occur for operating frequencies within the RFID UHF band. Finally, the good agreement between measurements and simulations demonstrates that our co-simulation method is an accurate modeling technique. 


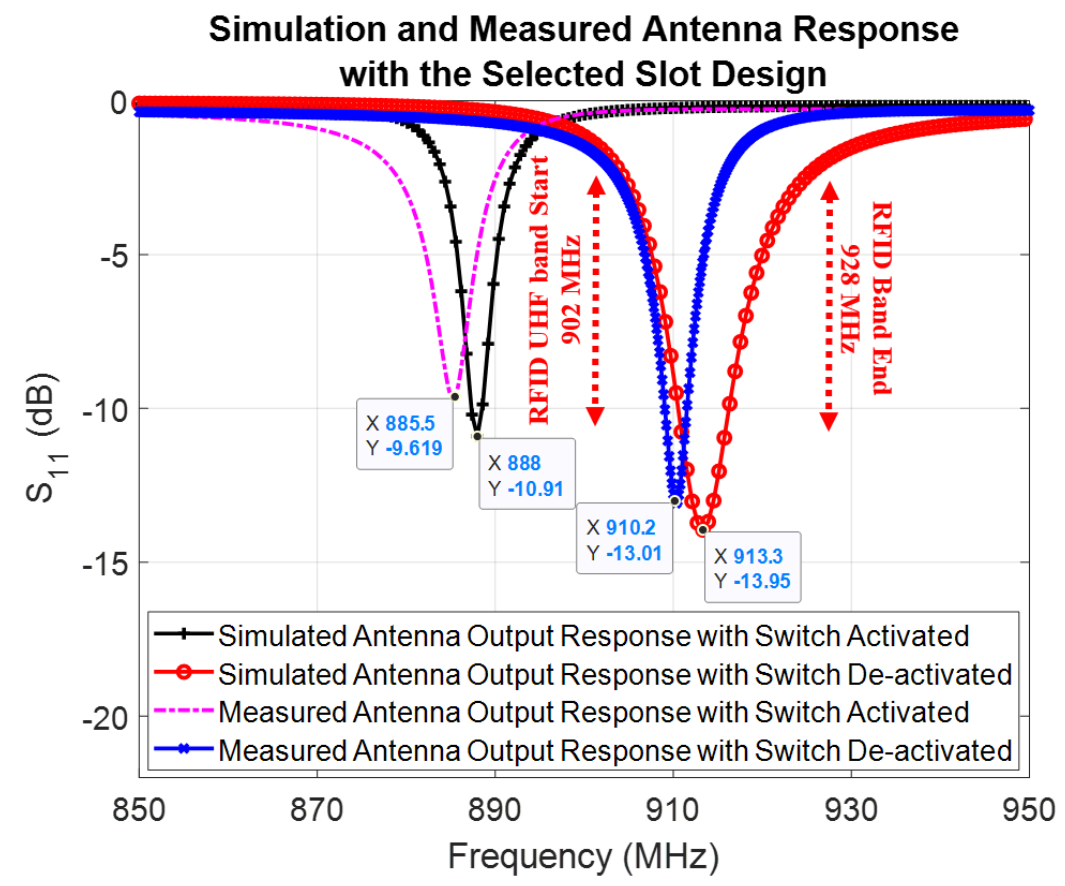

Figure 6.19. Measured and simulated results of RFID antenna with switch.

The slight discrepancy between the measured and simulated results is attributed to the tolerance associated with the Rogers TMM13i substrate permittivity [93].

\subsection{Matching Network Design}

Thus far, we have designed a passive antenna which switches operating frequencies based on temperature. The design consists of a switch, which is actuated by novel LCEs and a customized slotted patch antenna. To complete the sensor design, we must address the following design issues (refer to Figure 6.19): (a) the antenna currently operates outside the RFID band in one of the switch's states, as shown in Figure 6.19, and (b) the antenna impedance has not been matched to the conjugate of the RFID IC input impedance and this needs to be done at both the ON and OFF states of the switch. In this section, we discuss a two-stage matching network design that resolves these two issues. 


\subsubsection{First Stage of the Matching Network: Shifting the Switching Bandwidth}

It is helpful to first recognize that the antenna (see Figure 6.16) behaves as two different antennas on a single platform. Specifically, when the switch is in the deactivated state and the antenna operates at $910.2 \mathrm{MHz}$, and when the switch is in the activated state and the antenna operates at $885.5 \mathrm{MHz}$ (see Figure 6.19). To further express the dual behavior of this design, the input impedance of the antenna when the switch is in the deactivated and activated states is depicted in Figure 6.20 over the frequency range of 850$950 \mathrm{MHz}$. Also, the input impedances at the corresponding operating frequencies are labeled.

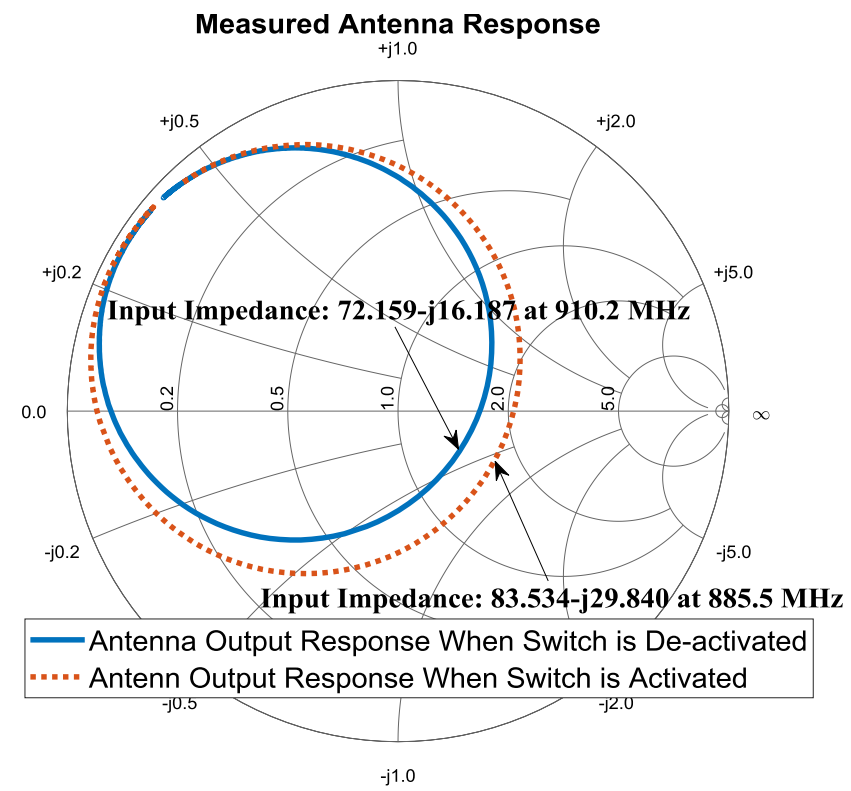

Figure 6.20. Measured input impedance of the fabricated antenna with the impedances at the current operating frequencies labelled.

The goal of the first stage of the matching network is to shift the switchingbandwidth of the design to within the RFID bandwidth. The technique used here, involves the matching of the input load impedance at the desired operating frequency (e.g., a frequency within the RFID band) to $50 \Omega$. However, since this design operates as two 
separate antennas, we must now apply this technique to two input impedance loads at the desired operating frequencies within the RFID UHF band, in this case at $927 \mathrm{MHz}$ when the switch is de-activated and $902 \mathrm{MHz}$ when the switch is activated. In other words, we would like to transform the input impedances at $927 \mathrm{MHz}$ for when the switch is deactivated and the input impedance at $902 \mathrm{MHz}$ for when the switch activated both to $50 \Omega$ using a single matching network (these operating frequencies are chosen as they are $25 \mathrm{MHz}$ apart similar to the inherent switching-bandwidth as depicted in Figure 6.19 and they are within the RFID UHF band). Furthermore, there exists a variety of techniques for matching complex loads at two different frequencies to a real load; however, they are only applicable for operating frequencies that are largely separated [99]-[103]. Therefore, these techniques are not suitable in this research, where the switching-bandwidth is relatively narrow (e.g., $25 \mathrm{MHz}$ ). Furthermore, since we cannot design a single matching network that operates simultaneously at both the chosen frequencies, we design the matching network using the average of the two frequencies (i.e., $(927+902) / 2 \approx 915 \mathrm{MHz})$. Since the bandwidth is relatively small $(25 \mathrm{MHz})$, this technique works well; however, with the consequence of losing a small portion of the switching bandwidth, as we will see later in this section. Figure 6.21 shows the input impedances at 927 and $902 \mathrm{MHz}$ at the deactivated and activated states of the switch, respectively. 


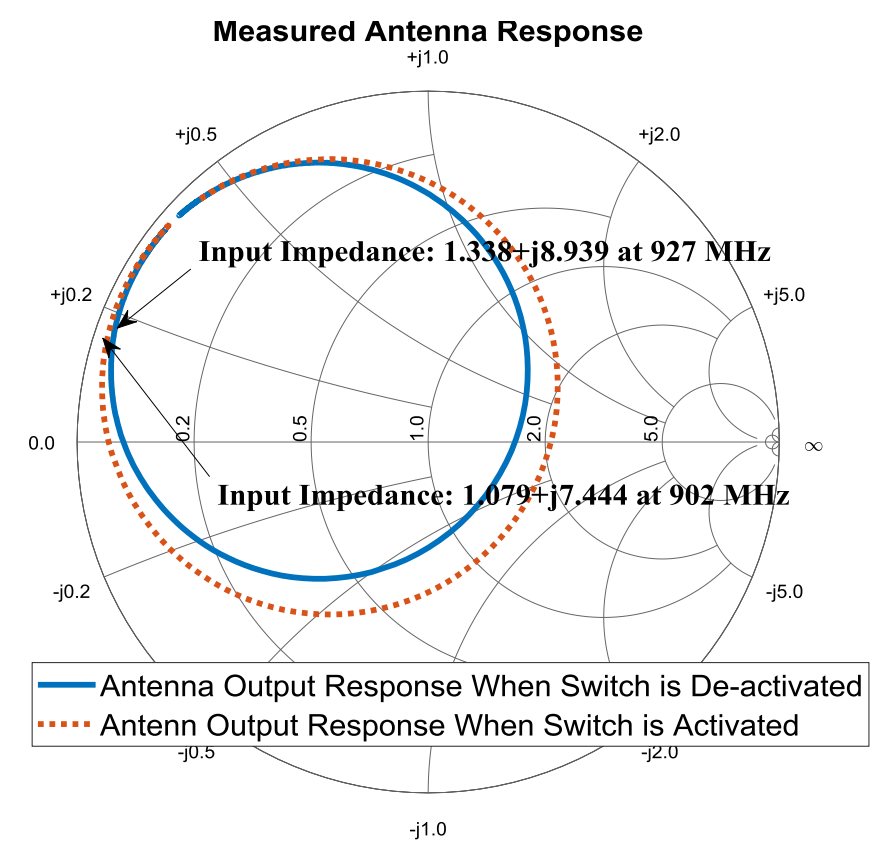

Figure 6.21. Measured input impedances of the fabricated antenna with the impedances at the desired operating frequencies labelled.

An important phenomenon takes place at these frequencies; that is, the complex input impedances are virtually identical. Conversely, if the two complex input impedances at 902 and $927 \mathrm{MHz}$ were greatly different, a single matching network that could then transform these impedances to a $50 \Omega$ load; simultaneously, would not be realizable. This is because, the proposed matching network is a permanent design which is fixed to transform one complex load to a $50 \Omega$ load. From Figure 6.21, the complex input impedance at the desired frequencies of operation are:

$$
\begin{aligned}
& 1.338+\mathrm{j} 8.939 \text { at } 927 \mathrm{MHz} \text { (switch deactivated) } \\
& 1.079+\mathrm{j} 7.444 \text { at } 902 \mathrm{MHz} \text { (switch activated) }
\end{aligned}
$$

Additionally, we use the average of the two impedance to account for the small difference. As mentioned earlier, since the matching network is a permanent design, it can only be configured to operate at a single frequency. Hence, we use the design frequency of 915 
MHz. To summarize, the goal is to design the first stage of the matching network to transform the complex input impedance of $1.209+\mathrm{j} 8.192 \Omega$ to a $50 \Omega$ load at $915 \mathrm{MHz}$.

Using the complex-input impedance criteria derived above, the matching network depicted in Figure 6.22 was designed.

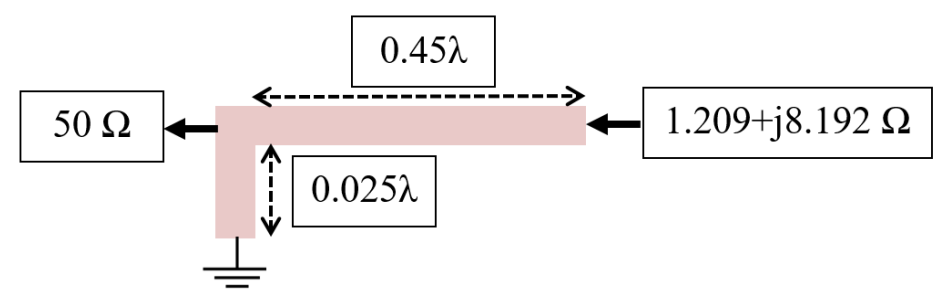

Figure 6.22. First stage of matching network. The input of $1.209+\mathrm{j} 8.192 \Omega$ at 902 and $927 \mathrm{MHz}$ is translated to $50 \Omega$ at 906.1 and $923.9 \mathrm{MHz}$, respectively (refer to Figure $6.23)$.

The output response at the $50 \Omega$ output is depicted in Figure 6.23. The frequency responses of the design have been shifted and now operate well within the RFID UHF band. Also, the responses are well matched to the $50 \Omega$ (in reference to a $50 \Omega$ port). That is, when the switch is de-activated the return loss is $-15.43 \mathrm{~dB}$, which is equivalent to $97 \%$ power transfer to the load. Additionally, when the switch is activated the return loss is $-17.52 \mathrm{~dB}$, which is equivalent to $98 \%$ power transfer to the load. Hence, these values indicate a favorable match to a $50 \Omega$ port. Conversely, as discussed earlier, the switching bandwidth has decreased to approximately $18 \mathrm{MHz}$; however, this bandwidth still remains substantial (70\% of the RFID UHF band). This happens because the matching network cannot support the simultaneous operation at 902 and $927 \mathrm{MHz}$. Hence, the matching network is designed to operate at the middle point (or average) of these frequencies instead. This provides a favorable match at two new operating frequencies with a sufficient switching bandwidth within the RFID UHF band as shown in Figure 6.23. 


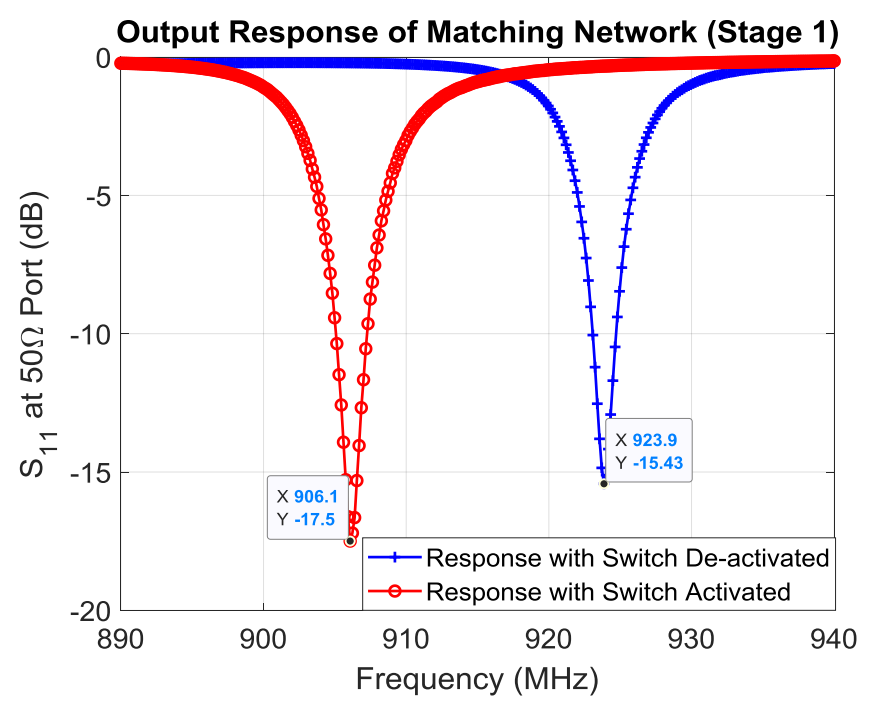

Figure 6.23. Simulated response of the output of the $1^{\text {st }}$ stage of the matching network.

\subsubsection{Second Stage of the Matching Network: Matching to the RFID IC}

The purpose of the second stage of the matching network is to match the $50 \Omega$ output responses (at the frequencies shown in Figure 6.23) of the first stage to the conjugate of the complex load of the RFID IC, i.e., $\mathrm{Z}_{\mathrm{IC} \_ \text {CONJ }}=18+\mathrm{j} 164 \Omega$. Similarly, to the design of the first-stage of the matching network, we design the second matching stage at the average frequency of the output response of stage 1 (from Figure 6.23), which is $915 \mathrm{MHz}$ $((923.9+906.1) / 2=915 \mathrm{MHz})$. The design of the second stage matching is shown in

Figure 6.24.

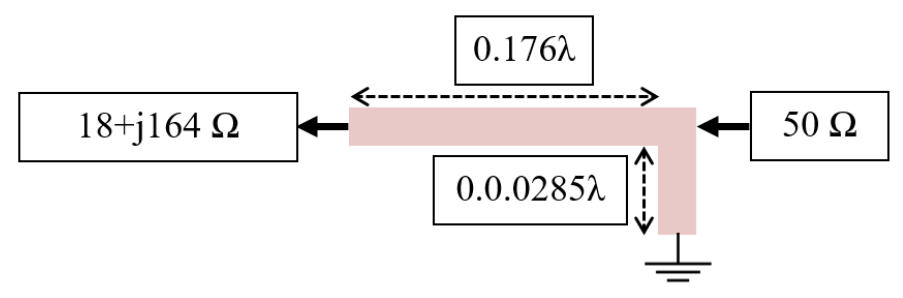

Figure 6.24. Second-stage of matching network. The input of $50 \Omega$ at 906.1 and 923.9 $\mathrm{MHz}$ (refer to Figure 6.23) is translated to the conjugate input impedance of the RFID IC at 902.2 and $921.1 \mathrm{MHz}$, respectively. 


\subsubsection{Final Impedance Matching Network Design and Optimization}

The final layout, when designed using a substrate with $\varepsilon_{r}=12.85$, of the matching network is depicted in Figure 6.25. This matching network transforms the antenna input impedance of $1.209+\mathrm{j} 8.192 \Omega$ (which occurs at both $910.2 \mathrm{MHz}$ and $885.5 \mathrm{MHz}$ for the deactivated and activated states, respectively) to the conjugate of the input impedance of the RFID IC of $\mathrm{Z}_{\mathrm{IC} \_ \text {CONJ }}=18+\mathrm{j} 164 \Omega$. The method presented here fulfills the impedance transformation successfully. However, there is room for improvement. Therefore, using the equation for the power transmission coefficient from (2.14) as a figure of merit, the electrical lengths of each transmission line segment, from Figures 6.22 and 6.24, were finely tuned to optimize the matching to the RFID IC. Specifically, the PTC and the switching bandwidth are maximized while ensuring that our tag will operate within the RFID UHF band. The final design of the tuned impedance matching network is shown in Figure 6.25. Also, the physical lengths of each transmission line segment can be obtained by using the midpoint design frequency of $915 \mathrm{MHz}$ since this frequency was used to design the $1^{\text {st }}$ and $2^{\text {nd }}$ stages of the matching network.

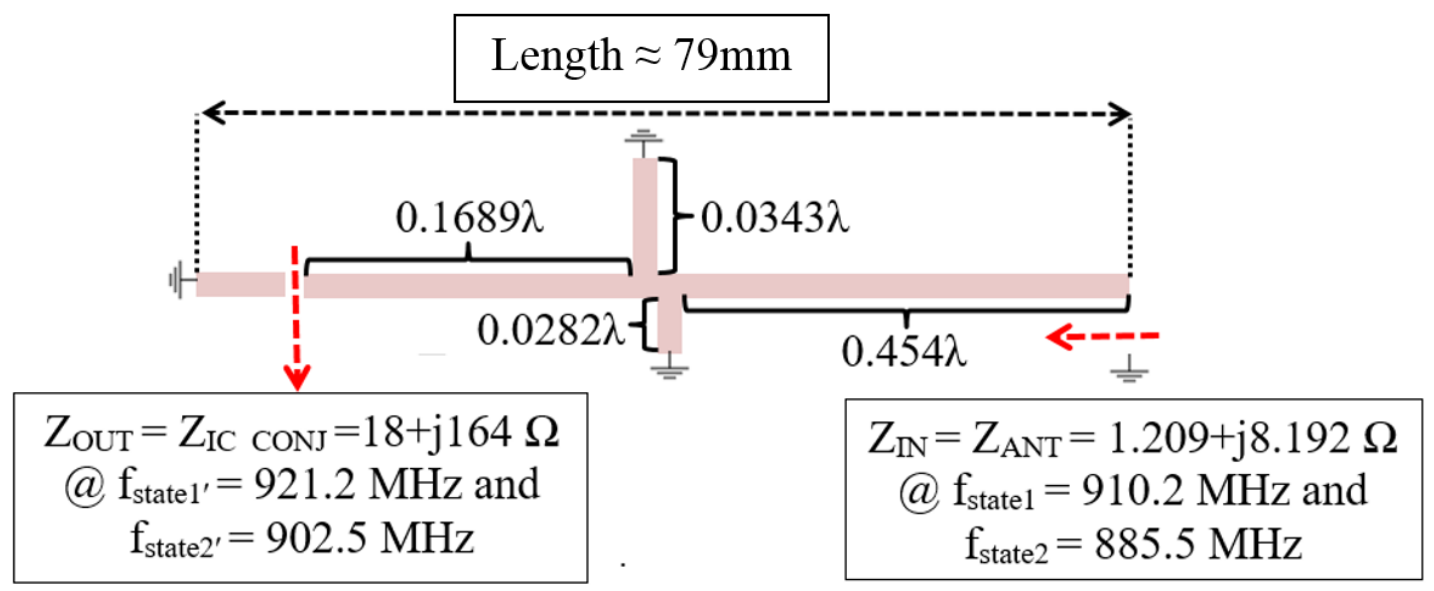

Figure 6.25. First and second stages of the matching network combined. 
The output response (PTC) of the final impedance matching network of Figure 6.25 is plotted in Figure 6.26.

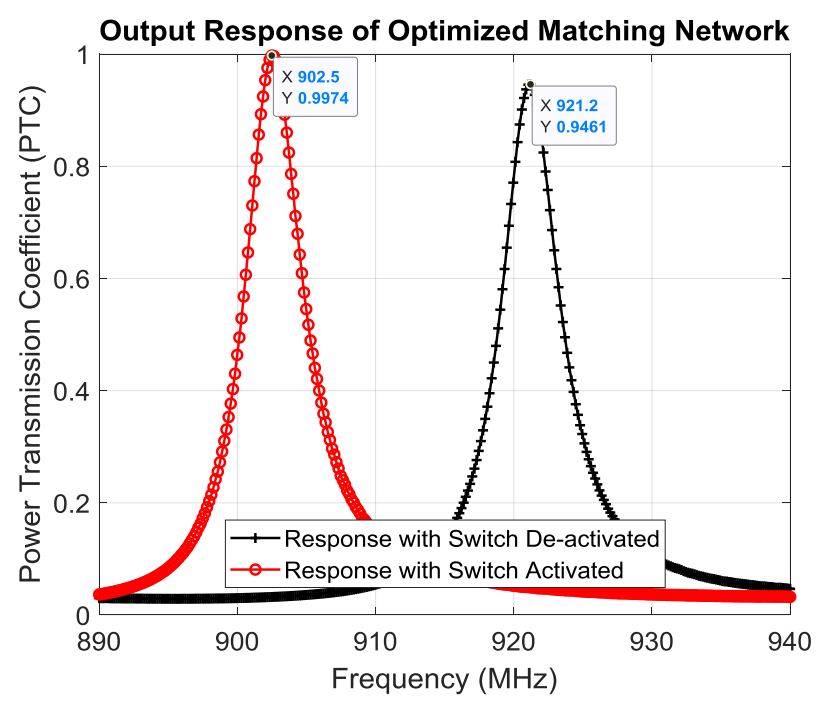

Figure 6.26. Simulated PTC of antenna with the final matching network.

According to Figure 6.26, the final operating frequencies for the deactivated and activated switch states now occur at 921.2 MHz and 902.5 MHz, respectively. Also, a favorable match to the RFID IC is achieved for both states of the switch as the PTC is higher than $90 \%$ at both operating frequencies. Finally, a switching bandwidth of approximately 19 $\mathrm{MHz}$ (or 73\%) of the RFID UHF band is achieved, which is a sufficient change to clearly indicate that a temperature threshold has been crossed.

According to Figure 6.25, the length of the proposed matching network (approximately $79 \mathrm{~mm}$ ) is not appropriate as it will protrude beyond the area of the patch antenna (see Figure 6.16) when it is connected to the feed port. As a result, the area of the matching network design was scaled down using a meandering technique [7, pp. 315-319]. Consequently, mitered bends and additional transmission line segments were added to complete the design. Once again, the electrical lengths of each transmission line segment were fine-tuned to maximize the switching bandwidth and matching to the RFID IC load 
while ensuring operability within the RFID UHF band. The layout is depicted in Figure 6.27.

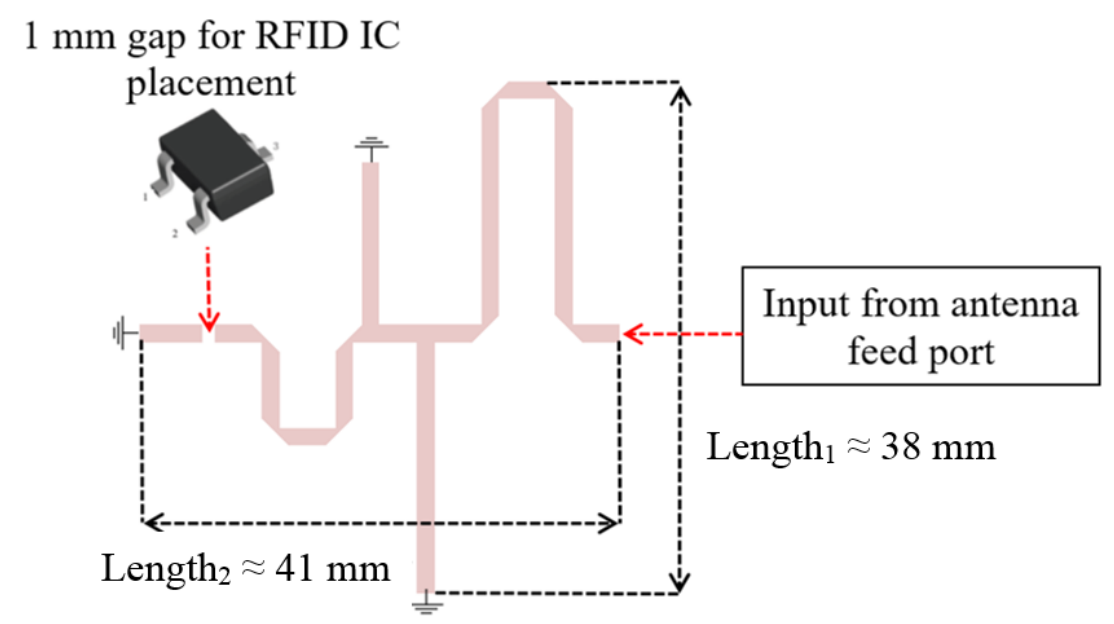

Figure 6.27. Final optimized and miniaturized matching network.

The simulated PTC of the antenna with the final meandered matching network is displayed in Figure 6.28.

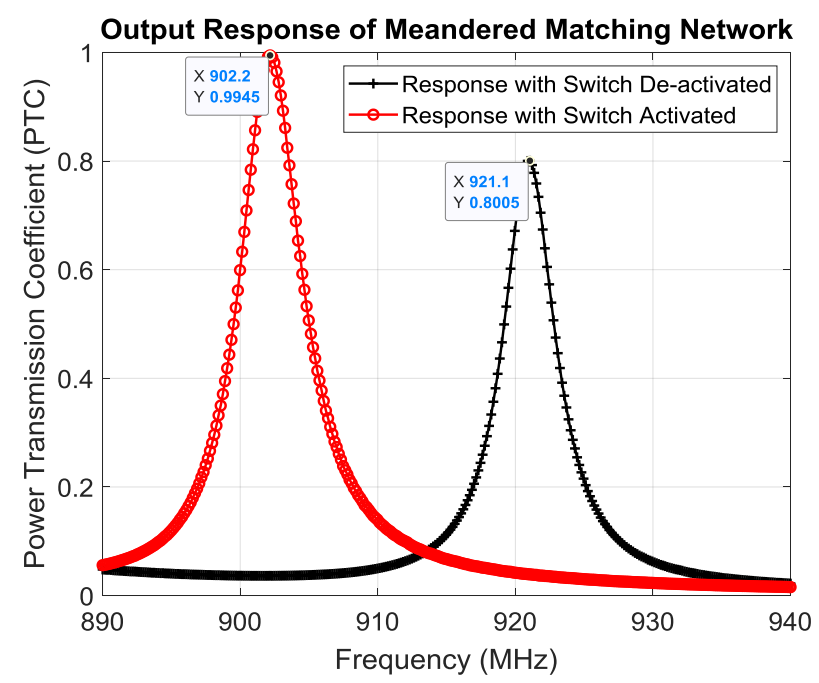

Figure 6.28. Simulated PTC of antenna with final matching network. The final operating frequencies are 921.1 MHz and 902.2 MHz at the OFF (de-activated or depressed) and $\mathrm{ON}$ states of the switch (activated or pressed), respectively.

According to Figure 6.28, the matching network achieves a maximum switching bandwidth of approximately $19 \mathrm{MHz}$. Moreover, the magnitude of PTC for both operating states is favorable; however, a slight decrease occurs in the PTC when the switch is deactivated 
compared to the design in Figure 6.25 whose performance is shown in Figure 6.26. Finally, due to the tuning of the transmission line segments, a slight shift in the operating frequencies for both states has occurred when compared to the performance curves of Figure 6.26. Therefore, the final operating frequencies of the sensor design in this research are as follows: $921.1 \mathrm{MHz}$ when the switch is de-activated and $902.2 \mathrm{MHz}$ when the switch activated.

\subsubsection{Impedance Matching Network Fabrication and Measurements}

Two versions of the final matching network design shown in Figure 6.27 were fabricated and they are depicted in Figure 6.29.

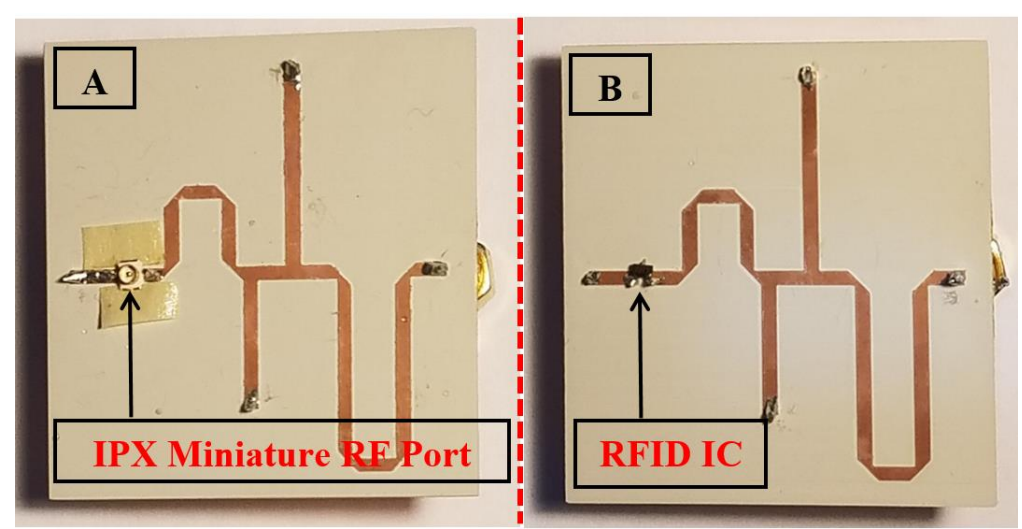

Figure 6.29. Two versions of the matching network are fabricated. (Left) An IPX port is connected for VNA measurement testing. (Right) An RFID IC is connected for RFID testing.

Version A was fabricated with an IPX miniature (male) port and version B incorporates the RFID IC. Version A is used to test the performance of the matching network using a VNA and version B is used to test the RFID performance of the sensor. The IPX port facilitates the direct connection to miniaturized circuit designs thereby providing results with minimal distortion due to phase error and parasitic capacitance (as compared to an SMA interface). Moreover, VNAs typically use coaxial test cables with an SMA interface, 
which cannot mate with the IPX port. To establish a connection from the VNA test cables, a coaxial cable with an SMA (female) interface on one-end and an IPX (female) port on the opposite end is utilized. However, with this SMA to IPX interface conversion cable, an accurate VNA calibration is necessary. To accomplish this, a customized calibration kit was fabricated. That is, three (male) IPX ports were converted into an open, short, and $50 \Omega$ terminations. For the open standard, the port was simply untouched. Additionally, for the short standard, the terminals of the port were soldered (i.e., shorted) together. Finally, for the $50 \Omega$ standard, a precision high-frequency surface mount resistor was connected between the terminals of the ports [104]. The custom calibration standards along with the VNA calibration setup are shown in Figure 6.30. 


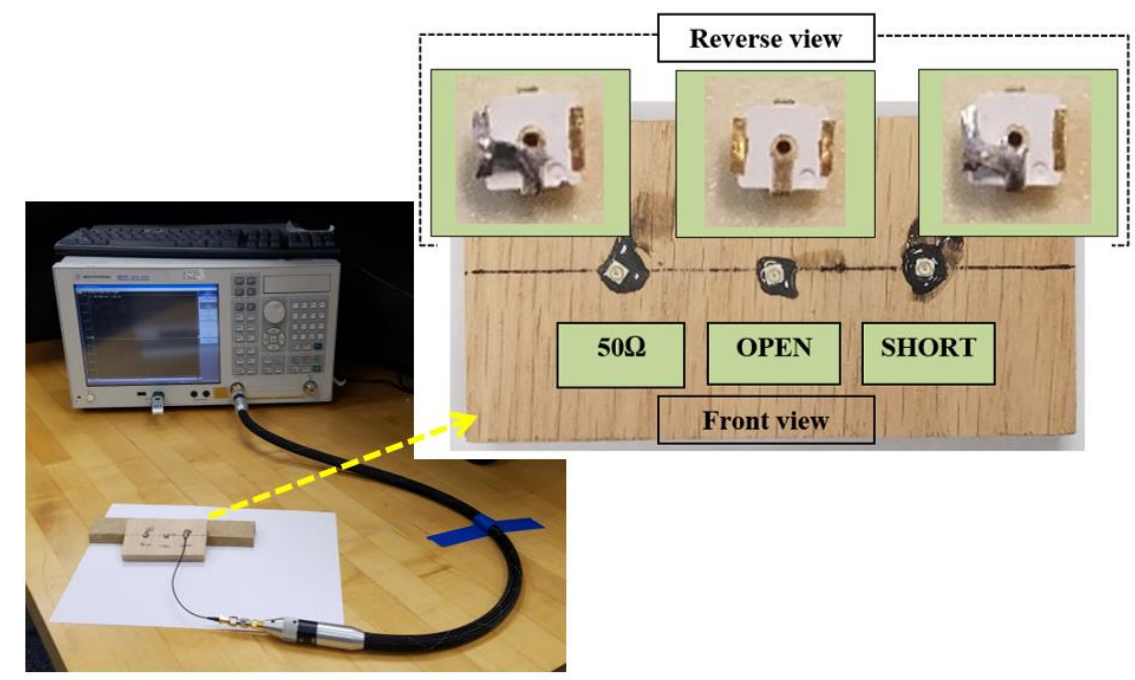

Figure 6.30. VNA setup with custom calibration standards for the IPX to SMA cable extension.

Once the calibration is performed, the IPX cable is attached and secured to the fabricated matching network, which is then connected to the antenna, refer to Figure 6.31.

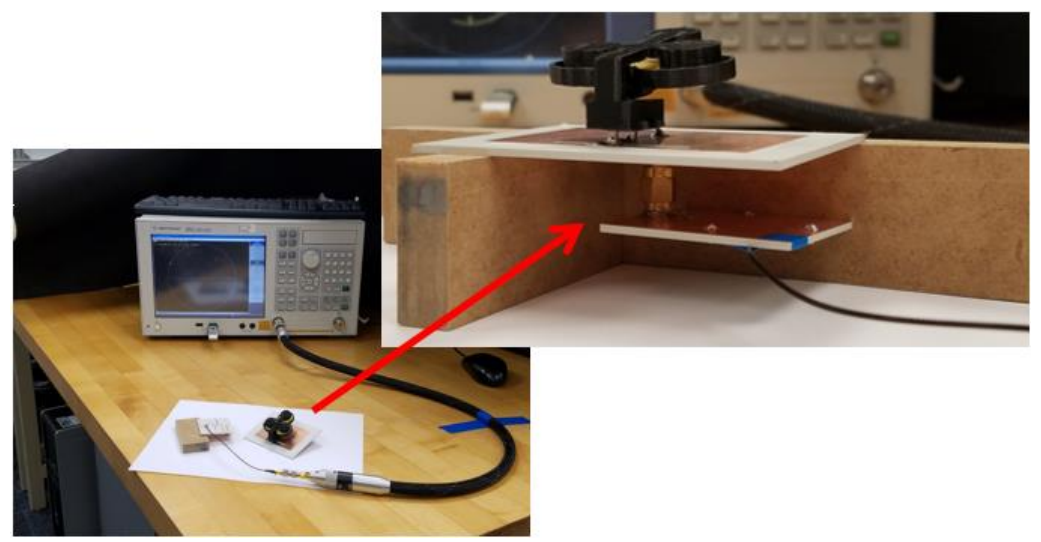

Figure 6.31. The impedance matching network with the IPX port is connected to the antenna. The performance of the design is tested using the VNA.

After taking the VNA measurements (for both activated and deactivated switch states) and using (2.14), the corresponding PTCs are calculated and plotted in Figure 6.32. 


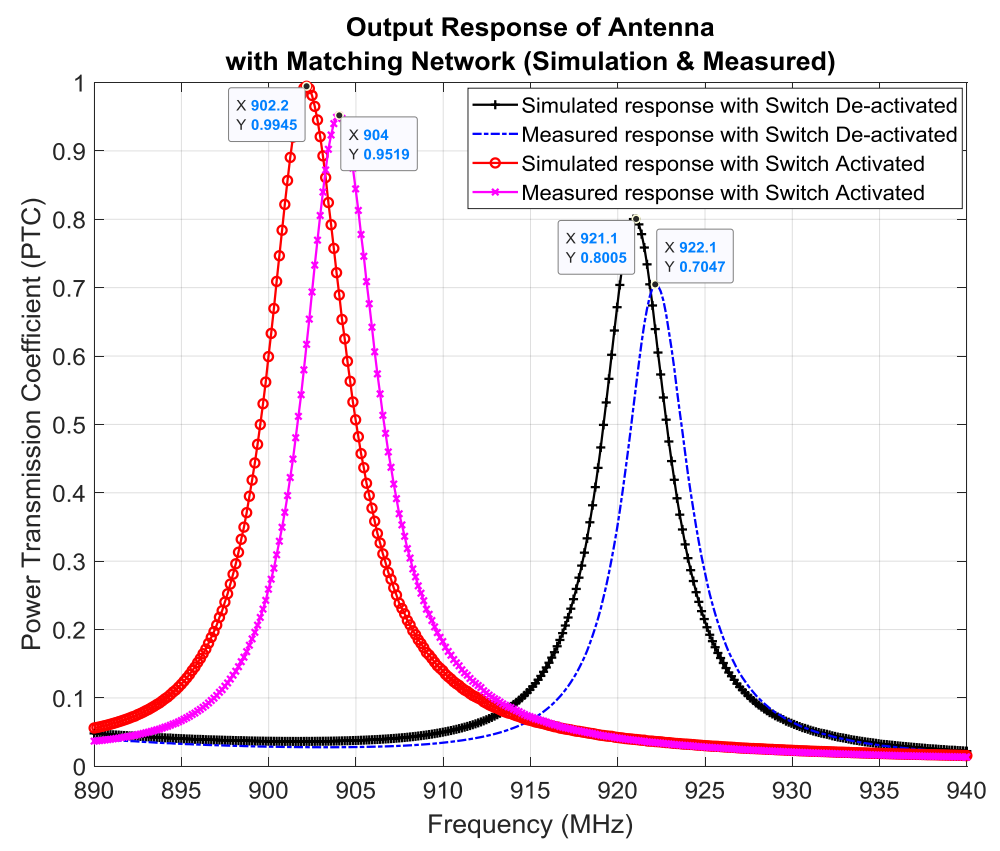

Figure 6.32. Measured and simulated PTC of the antenna with matching network. When the switch is OFF (not pressed) the measured antenna operates at $922.1 \mathrm{MHz}$. When the switch is ON (pressed) the measured antenna operates at $904 \mathrm{MHz}$.

The measured results agree well with the simulated ones. However, in the measured results, there exists a slight shift in the operating frequencies $(904 \mathrm{MHz}$ when the switch is deactivated and 922.1 MHz when the switch activated) along with a small drop of the PTC. This is attributed to the male SMA PCB connector used to connect the matching network to the antenna (which could not be accounted for in the calibration process) and due to the tolerance associated with the Rogers TMM13i substrate, which was used to design both the antenna and matching network [93]. Finally, the measured input impedances for both switch states are plotted and labeled using the Smith chart in Figure 6.33. These measured impedance values show promising results as they close to the conjugate of the input impedance of the RFID IC (i.e., $\mathrm{Z}_{\mathrm{RFID} \mathrm{IC}}=18-\mathrm{j} 164 \Omega$ ). 


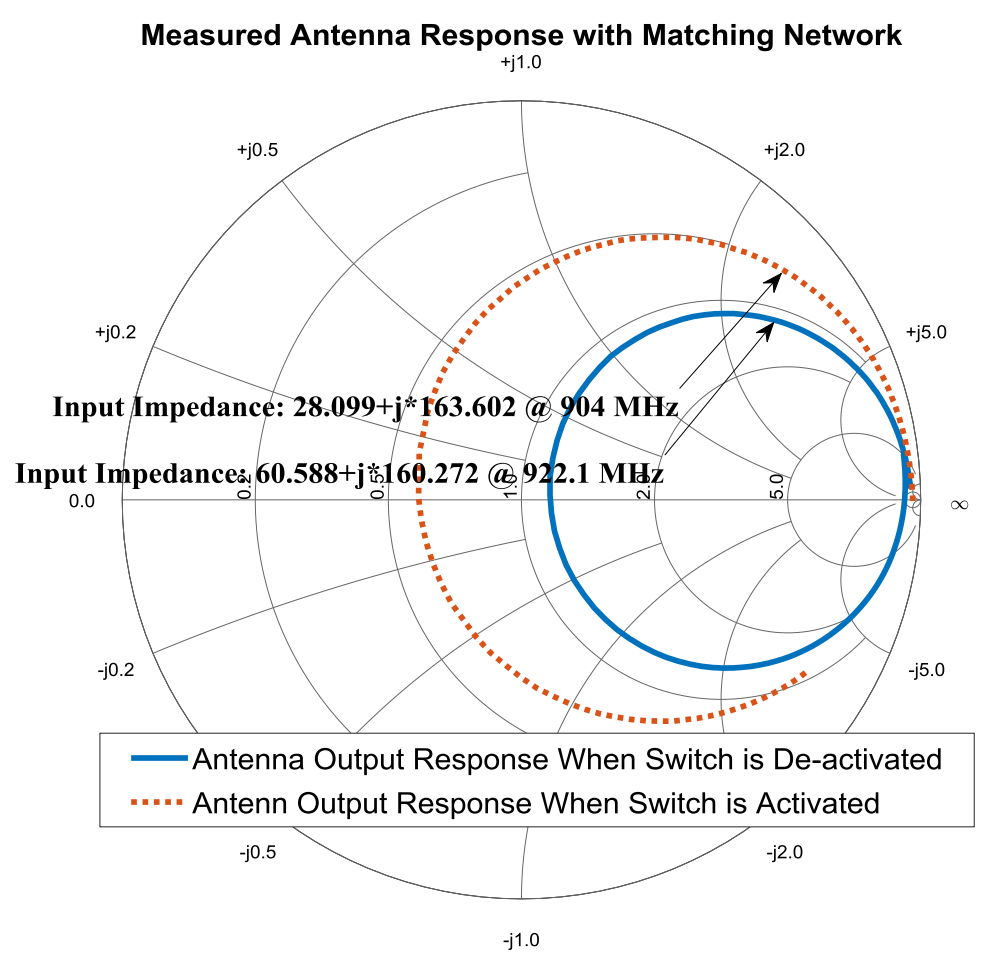

Figure 6.33. The measured input impedance of antenna with switch and matching network at both states of the switch.

\subsection{Final Design and RFID Performance Testing}

From Section 6.3 and 6.4, the design of the frequency switching antenna and customized matching network is discussed and confirmed through experimentation, respectively. The RFID temperature sensor is completed by combining the designed antenna (from Figure 6.16) with the fabricated matching network, which includes the RFID IC that is shown in Figure 6.29 (right). Moreover, an enclosure is fabricated to support the antenna structure and the complete sensor is depicted in Figure 6.34. 


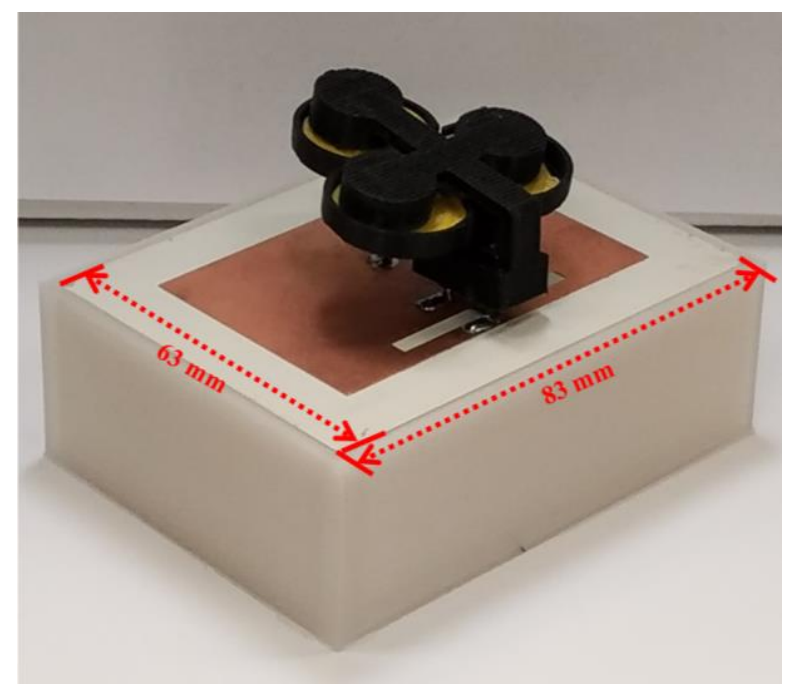

Figure 6.34. Passive RFID temperature sensor.

To test the performance of the proposed sensor, three experiments are conducted. In the first one, we use the ThingMagic Pro RFID reader to test the frequency shifting capability of the proposed sensor at the two states of the switch (i.e., in this static test, the switch is actuated manually). In the second one, we validate the temperature-dependent frequency shifting capability of the antenna being actuated by the cold-responsive LCE. In the third one, we perform RFID measurements with the proposed sensor in a coldtemperature environment to demonstrate operation in actual working conditions. Finally, in the first and last experiments, the test is repeated three times to validate the operational consistency of the proposed sensor design. 
A conceptual diagram of the RFID measurement setup is depicted in Figure 6.35.

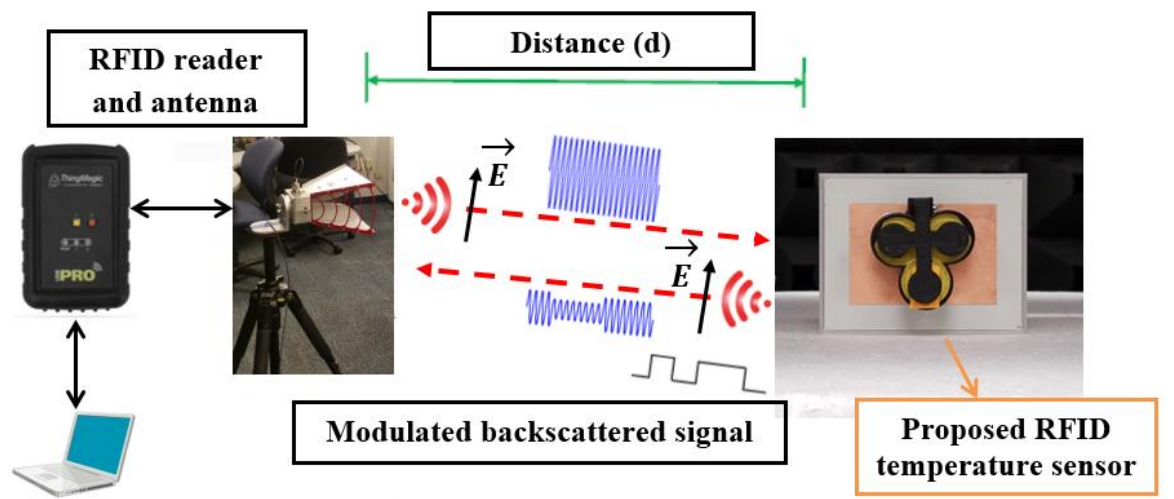

Figure 6.35. Diagram of the RFID measurements setup.

From Figure 6.35, a linearly polarized double-ridge horn antenna with $7 \mathrm{dBi}$ gain is connected to the RFID reader [77]. Both the reader and the proposed sensor (linearly polarized) are oriented with vertical polarizations. Finally, the procedure for taking RFID measurements is the same as discussed in Section 5.7. Firstly, the power transmitted from the RFID reader is increased in increments of $0.1 \mathrm{dBm}$ until the threshold power of the RFID IC is reached. The threshold power is identified as the minimum power level at which data begins to be retrieved from the temperature sensor [7, pp. 70-77], [35], [78]. Secondly, the transmitted power at which the developed sensor responds is recorded and this procedure is conducted at every frequency in increments of $1 \mathrm{MHz}$ in the 902 to $928 \mathrm{MHz}$ band. Finally, the operating frequency is the one where the least transmitted power is required to turn on our RFID tag.

\subsubsection{RFID Static Testing}

This RFID static test is conducted to demonstrate the frequency switching capability of our sensor using an RFID reader. Consequently, the switch in this case only needs to be 
actuated manually. The RFID test setup is shown in Figure 6.36, where the developed sensor is placed $0.762 \mathrm{~m}$ directly in line-of-sight of the reader antenna.

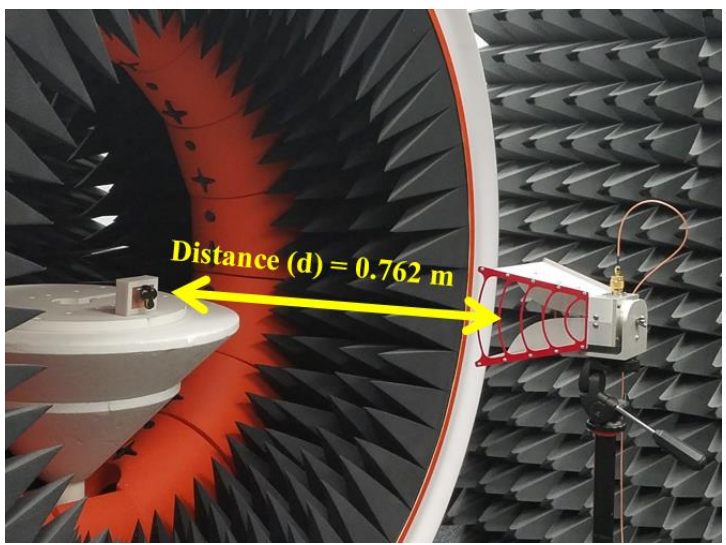

Figure 6.36. Static RFID testing.

Furthermore, the measurements were taken three times for each switch state. The measured results when the switch is at the OFF state (not pressed or deactivated) are shown in Figure 6.37 .

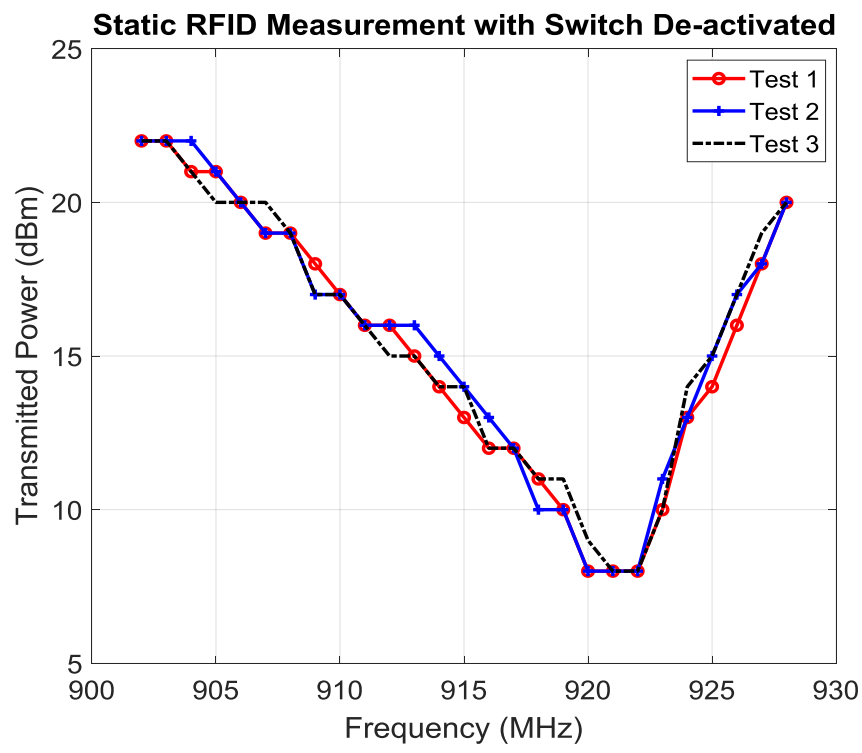

Figure 6.37. Static RFID measurement results with the switch at the OFF (deactivated) state.

It is seen that the frequency at which the proposed sensor responds with the minimum transmitted power is at approximately $921 \mathrm{MHz}$, which agrees with the results of Figure 
6.32. The measured results when the switch is at the ON state (pressed or activated) are shown in Figure 6.38.

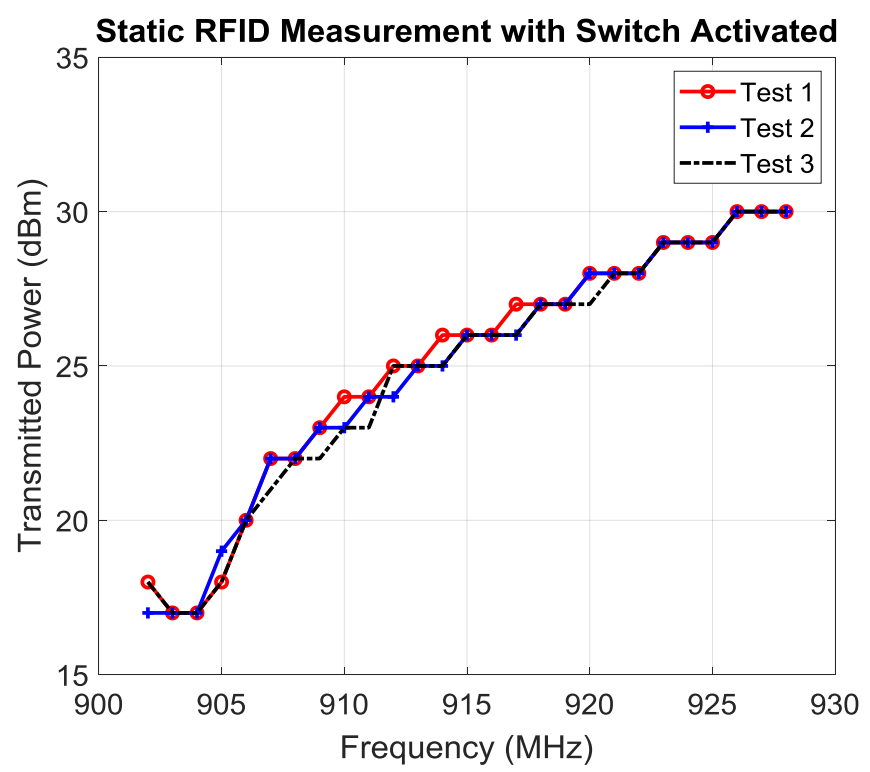

Figure 6.38. Static RFID measurement result with the switch in the ON (activated) state.

It is seen that the frequency at which the proposed sensor responds with the minimum transmitted power is at approximately $902 \mathrm{MHz}$, which again agrees with the results of Figure 6.32.

Furthermore, the PTC plots in Figure 6.32, show that better matching is achieved when the switch is activated. However, according to Figure 6.38, the associated transmitted power required to turn on and read our sensor is higher when the switch is activated. This is expected because the PTC curves in Figure 6.32 do not account for the radiation efficiency of the antenna. In fact, according to [105], the radiation efficiency is linearly related to the frequency for a rectangular patch antenna on an electrically thin substrate (with substrate thickness of approximately $1.27 \mathrm{~mm}$ and permittivity of 12.85 ). That is, as frequency increases the radiation efficiency increases. Therefore, more transmitted power is required to turn on and read the sensor for the activated switch-state since it operates at 
a lower frequency (Figure 6.38); even though the matching is better at the activated switchstate (Figure 6.32).

\subsubsection{Frequency Shifting Using the LCE Array}

In this test, we expose the antenna with the LCE and its support (as shown in Figure 6.17) to cold temperatures to demonstrate the switch's actuation by the proposed LCE array (refer to Figure 4.9). Therefore, in this experiment the matching network was not needed and the measurements were taken directly from the antenna output port. First, the antenna was connected to an Agilent N9923A FieldFox Vector Network analyzer and a measurement was taken at room temperature. This setup is depicted in Figure 6.39 where the recorded temperature was $29^{\circ} \mathrm{C}$ (i.e., $84^{\circ} \mathrm{F}$ ) at the operating frequency of $910 \mathrm{MHz}$.

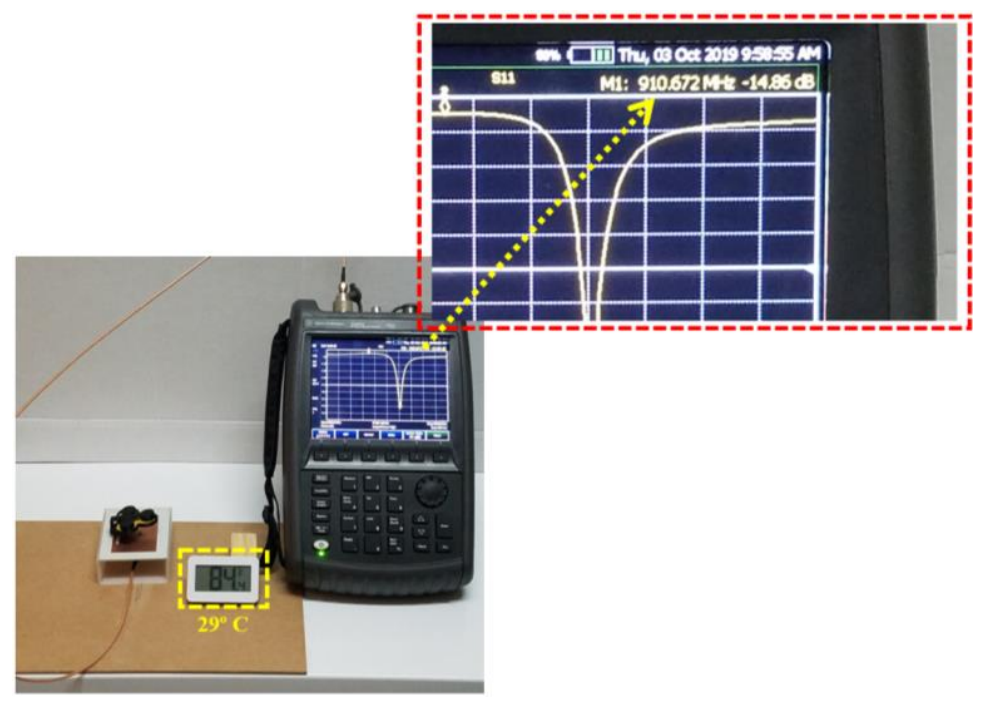

Figure 6.39. The LCE array is relaxed at $29^{\circ} \mathrm{C}$. The operating frequency of the antenna design is $910 \mathrm{MHz}$.

Subsequently, the antenna was disconnected from the VNA and it was placed inside a freezer along with a digital thermometer. When the temperature threshold of the LCE was reached (e.g., approximately $-9^{\circ} \mathrm{C}$, refer to Section 4.4 ), the antenna was removed from the 
freezer and re-connected to the VNA. This setup is shown in Figure 6.40, where the recorded temperature was $-9^{\circ} \mathrm{C}$ (i.e., $\left.16^{\circ} \mathrm{F}\right)$.

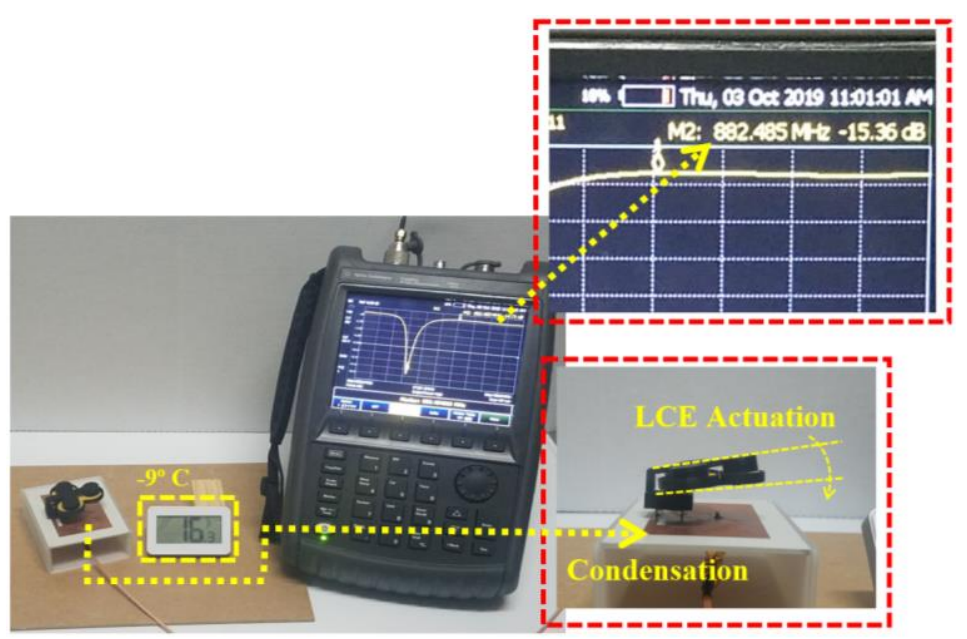

Figure 6.40. The LCE array is actuated at $-9^{\circ} \mathrm{C}$. The switch has been triggered and the operating frequency of the antenna design is $882 \mathrm{MHz}$

The measurements from Figures 6.39 and 6.40 confirm that the LCE successfully actuates and triggers the switch, which causes the operating frequency of the tag to shift from $910 \mathrm{MHz}$ to $882 \mathrm{MHz}$. It is important to note that due to the cold surface of the antenna cooling the surrounding air, condensation is formed on the metallic patch [106]. As a result, in reference to Figure 6.19, this caused a slight shift in the initial operating frequency, which was $885 \mathrm{MHz}$. Furthermore, as the LCE returns to its relaxed state, the switch automatically deactivates, and the antenna returns to its initial operating frequency, as shown in Figure 6.39.

\subsubsection{RFID Dynamic Testing}

In this experiment, we expose the developed sensor to cold-temperatures and perform an RFID measurement. The purpose of this measurement is to demonstrate the successful operation (automatic frequency switching ability using an RFID reader) of the 
sensor in realistic environmental conditions. Similarly, to the procedure in the previous section, the sensor is placed inside a freezer until the temperature reaches (or exceeds) the morphing temperature of the LCE which is between $-9^{\circ}$ to $-12^{\circ} \mathrm{C}$ as detailed in Section 4.4. Subsequently, the sensor is placed on the test platform, as shown in Figure 6.41.

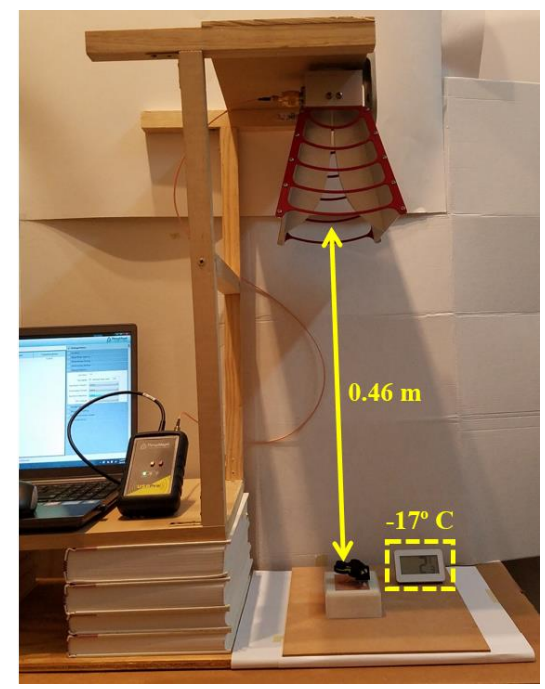

Figure 6.41. Dynamic RFID setup with the designed sensor at $-17^{\circ} \mathrm{C}$.

In this case, the LCE is cooled to $-17^{\circ} \mathrm{C}$ and the actuation of the LCE array triggers the switch. The corresponding RFID measurements indicate that the sensor responds at 902 $\mathrm{MHz}$ as the transmitted power is minimum at this frequency, refer to Figure 6.42 . 


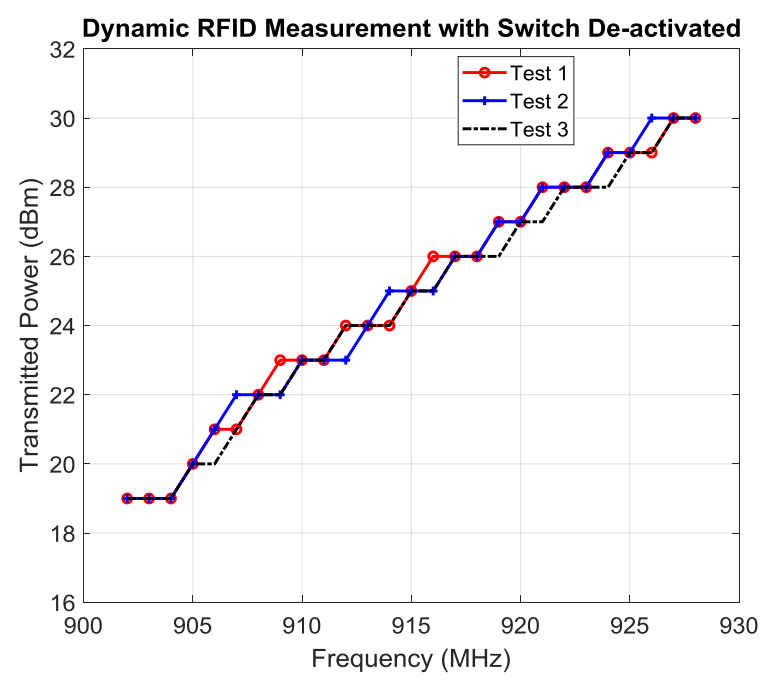

Figure 6.42. Dynamic RFID measurement results when the switch in the ON (triggered) state.

Shortly after, as the temperature raises to the ambient temperature of $28^{\circ} \mathrm{C}$, the LCE returns to its relaxed state (without any external intervention), refer to Figure 6.43.

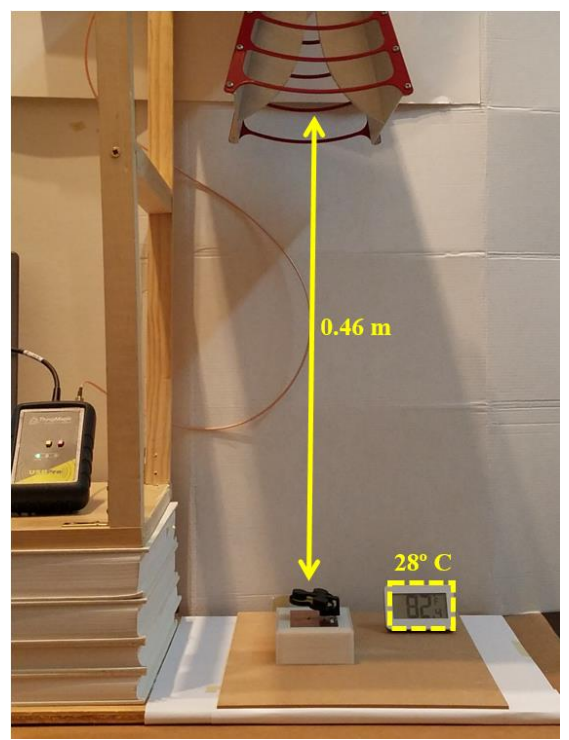

Figure 6.43. Dynamic RFID setup with the designed sensor at $28^{\circ} \mathrm{C}$.

Consequently, the corresponding RFID measurements indicate that the minimum transmitted power required to receive a response back from the proposed sensor is at approximately $921 \mathrm{MHz}$ (see Figure 6.44). 


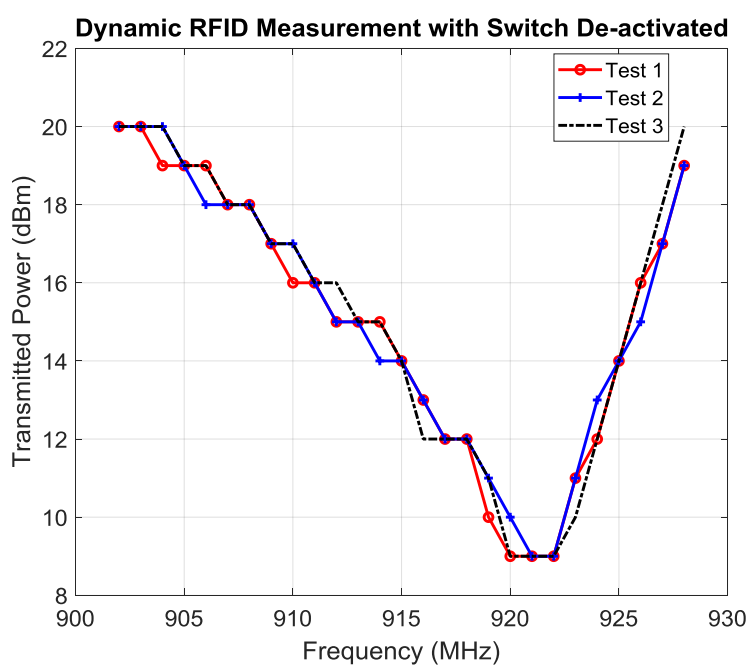

Figure 6.44. Dynamic RFID measurement results with the switch OFF (deactivated) state.

These dynamic RFID measurements agree well with the static RFID measurements from Section 6.5.1 (refer to Figures 6.37 and 6.38). Also, these measurements agree with the VNA measurements shown in Figure 6.32. Moreover, these measurements indicate that the design methodology (e.g., the patch antenna with slot that is integrated with a switch and a matching network) works correctly. Notably, the novelty of this passive sensor lies on its autonomous (i.e., no intervention is required), passive (i.e., battery-free), and continuous (i.e., operates through many temperature cycles) operation, which is achieved using novel cold-responsive programmable and reversible LCE technology to actuate the switch.

\subsection{Conclusion}

In summary, a new sensor has been designed, fabricated, and tested which conveys changes in temperature through controlled switching of its operating frequency within the RFID UHF band. The proposed sensor consists of a patch antenna with an integrated slot and a mechanical switch that is connected across the slot. Moreover, the switch is activated 
using state-of-the-art 4D printed cold-responsive LCEs. The LCE actuator is designed in a triangular array configuration to provide maximum force.

Specifically, when a temperature threshold violation occurs (i.e., at temperatures near $-9^{\circ} \mathrm{C}$ ), the operating frequency of the sensor shifts. Also, when the temperature is restored (i.e., to room-temperature near $29^{\circ} \mathrm{C}$ ), the operating frequency is restored as well without any intervention. Notably, this sensor has the capability to operate through multiple (room-to-cold and cold-to-room) temperature cycles thereby providing critical information about the temperatures that products are exposed to in the cold supply chain. 


\section{CHAPTER 7}

\section{IC (EPC) SWITCHING BOW-TIE ANTENNA DESIGN}

\subsection{System Overview}

This RFID passive temperature sensor functions by switching operation between two RFID ICs based on the temperature. As detailed in Section 2.5, the internal memory of each RFID IC contains a unique identifying 96-bit Electronic Product Code (EPC) [8]. Therefore, based on this unique identifier, two different temperature thresholds can be clearly detected by switching between two RFID ICs. The functionality of the proposed sensor is best summarized by the diagram shown in Figure 7.1.

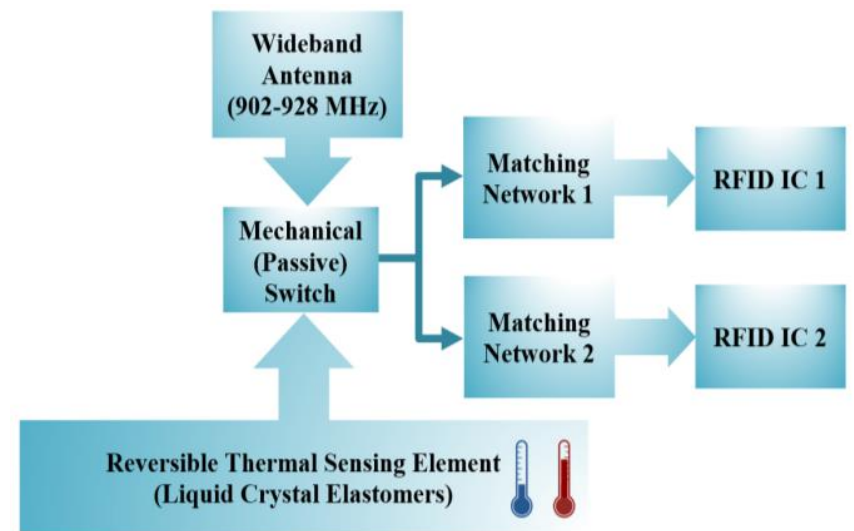

Figure 7.1. Operational diagram of the proposed temperature sensor.

The tag consists of the following three components: the antenna with its balun, a three-port mechanical (passive) switch, and a set of matching networks.

The proposed sensor consists of a wideband antenna that operates in the RFID UHF band of 902 to $928 \mathrm{MHz}$. The output of the antenna is fed into a mechanical light-duty switch, which is utilized to keep the design purely passive [92]. Moreover, novel coldreactive liquid crystal elastomers are utilized to reversibly trigger the switch upon reaching a temperature threshold. Furthermore, in the relaxed state (at room temperature) the switch 
is configured to activate the first RFID IC (RFID IC 1). When a temperature threshold is reached, the LCE morphs into a cone shape and activates the switch. As a result, RFID IC 1 is then turned OFF and RFID IC 2 is turned ON. Therefore, by switching between the two RFID ICs, one can easily assess whether a temperature threshold has been reached. Furthermore, since the LCE array returns to its original form when the stimulation is removed, the sensor can operate continuously through multiple temperature cycles without the use of any batteries.

\subsubsection{Antenna and Feed Structure}

In this sensor design, a microstrip coplanar bow-tie antenna is used. This antenna is a lightweight and practical derivative of the infinite biconical antenna [33, pp. 487-496]. Furthermore, the bow-tie antenna retains the wideband constant input-impedance characteristic of the biconical antenna. Specifically, the input impedance of the bow-tie antenna depends on its flare angle and according to Rumsey's principle, an antenna is frequency-independent if the antenna shape can be specified in terms of angles [34, pp. 380-387]. To design the bow-tie antenna for the RFID UHF band, we used the equations

presented in [33, p. 494]. Then, the design was optimized by using simulation analysis, which was performed in ANSYS HFSS.

Furthermore, due to the symmetry of the bow-tie dipole, this antenna is balanced [43, p. 460]. As a result, the feed structure consists of a single-input $50 \Omega$ microstrip transmission line connected to a quarter-wave transformer. The output of this transformer behaves as a $\mathrm{T}$-junction and transitions into a pair of $50 \Omega$ microstrip transmission lines. Hence, this transformer will translate a $50 \Omega$ transmission line impedance into a $35.4 \Omega$ 
transmission line load. The pair of microstrip transmission lines are then designed with a phase difference of $180^{\circ}$. This phase difference generates an odd-mode current excitation, which can be naturally transferred to the symmetric coplanar strip-line (CPS) that feeds the coplanar bow-tie antenna [107]-[110]. The CPS was designed based on the closed-form equations in [95, pp. 257-280] and then optimized using simulations. The final length of the CPS is $0.365 \lambda_{g}(33.35 \mathrm{~mm})$. The ratio of the substrate-height to the trace-width is 0.907 and the space between the traces of the CPS is $0.1 \mathrm{~mm}$. Based on the equations and design plots presented in [95, pp. 257-280], these dimensions provide a CPS with a characteristic impedance of approximately $50 \Omega$. Finally, an optimized design using Rogers TMM13i substrate with relative permittivity of 12.85 was simulated [93]. The antenna and its feed network are shown in Figure 7.2.

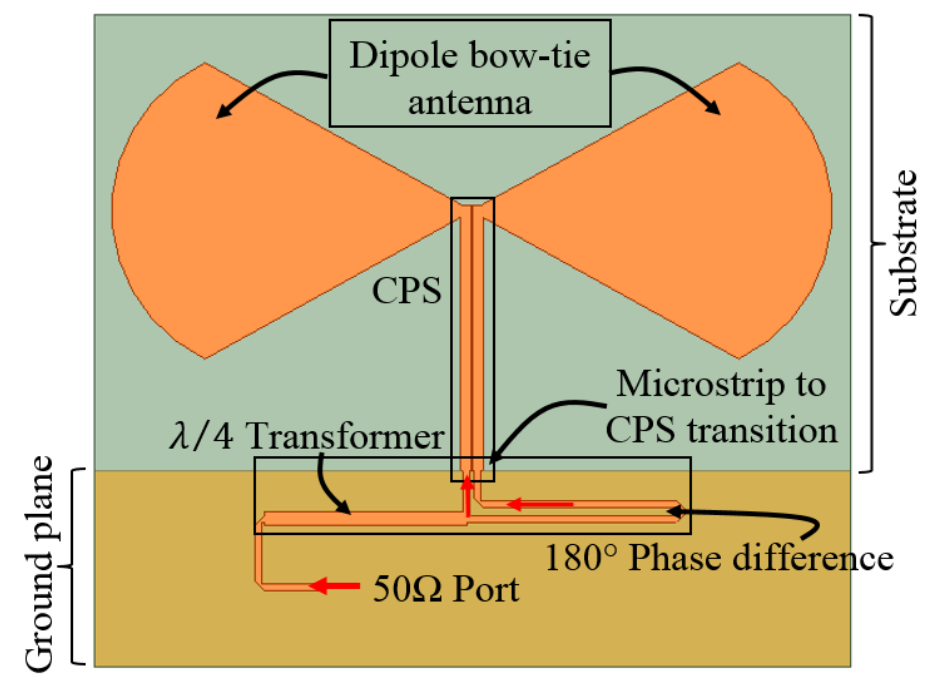

Figure 7.2. Bow-tie antenna design with feed network.

Figure 7.3 illustrates the simulated 3-D radiation pattern, which shows that the antenna provides $360^{\circ}$ elevation-plane coverage. This is advantageous over patch antenna designs, which only provide coverage in a half-plane opposite to the ground plane. 


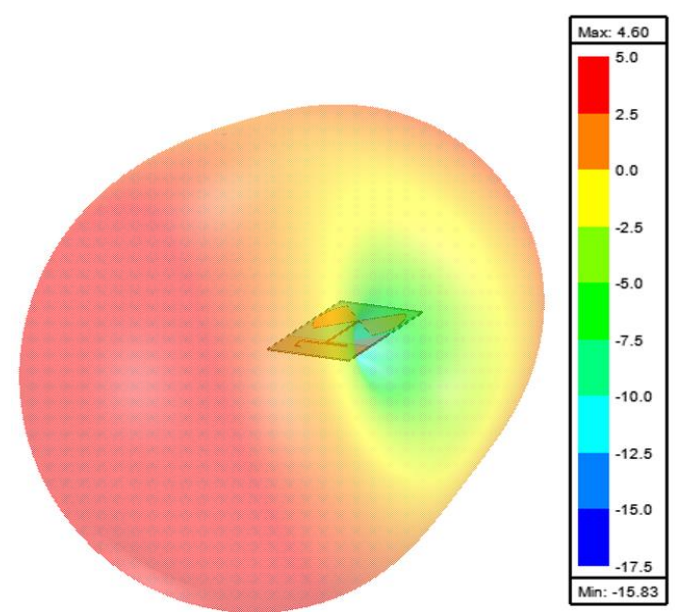

Figure 7.3. Antenna radiation pattern (realized gain in $\mathrm{dBi}$ ) showing $360^{\circ}$ coverage in the elevation-plane.

The proposed antenna design along with its feed network was fabricated and tested as shown in Figure 7.4.

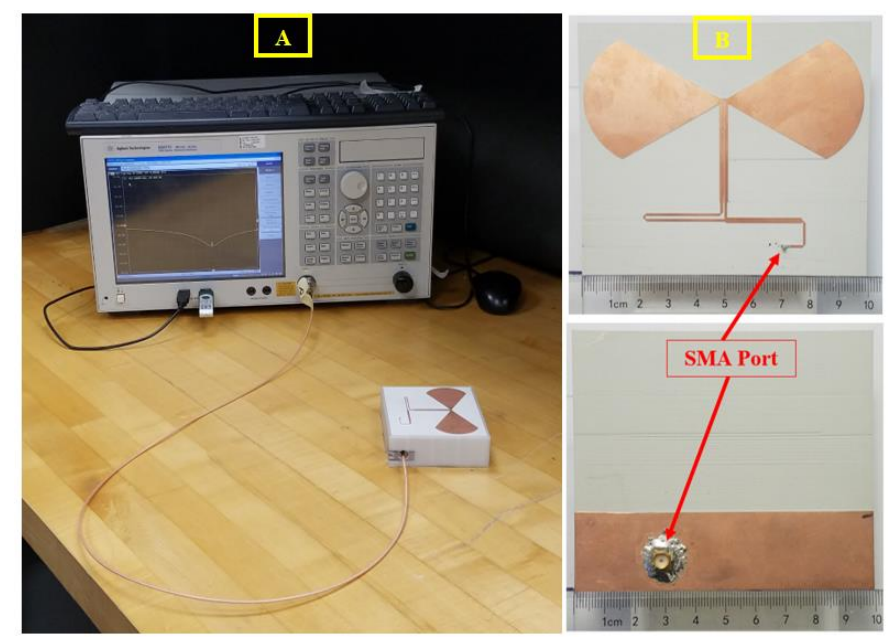

Figure 7.4. VNA test setup with fabricated antenna.

An SMA connector was soldered to the input of the design. Moreover, an enclosure was designed to elevate the antenna from any resting plane. This elevation prevents the detuning of the antenna when it is placed on various surfaces, such as, wood, plastic, and cardboard. The measured and simulated responses of the antenna are plotted in Figure 7.5. 


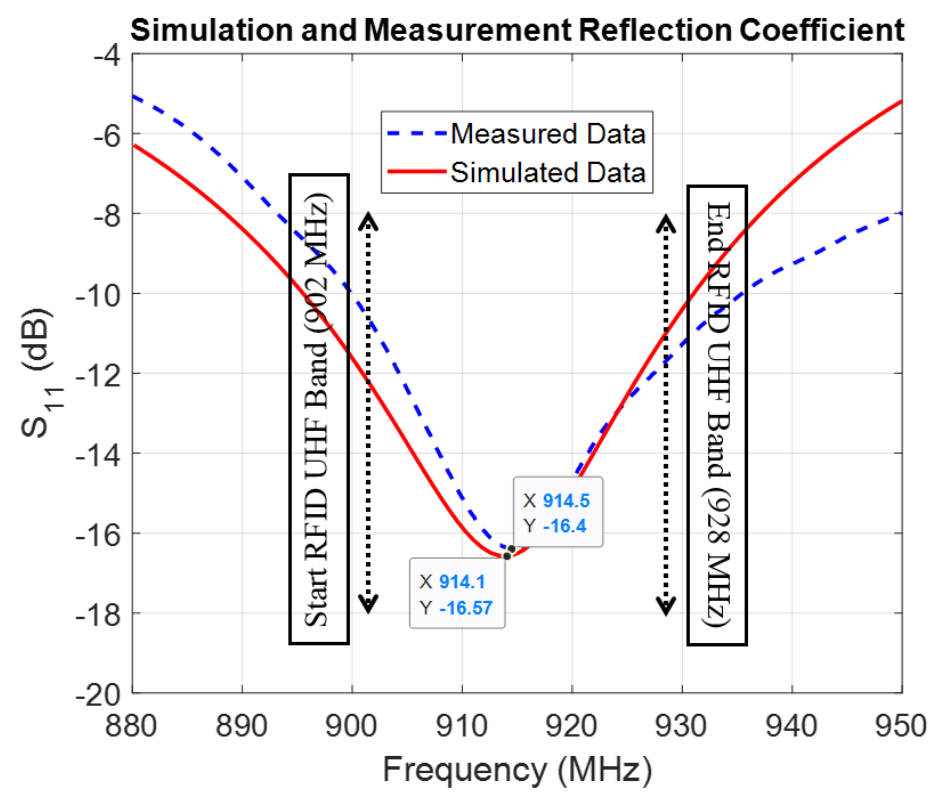

Figure 7.5. Measured and simulated S11 of the proposed antenna.

The measurement and simulated results agree very well. Also, the antenna provides a good impedance match over the UHF RFID bandwidth, i.e., the reflection coefficient is less than -10 dB from 902 to $928 \mathrm{MHz}$ (this equates to more than $90 \%$ power transfer within the frequency-band).

\subsection{Three-Port Switch Model}

In reference to Figures 7.1 and 7.2 , the $50 \Omega$ port feeds into a passive switch. In this sensor design, a light-duty mechanical single-pole-double-throw switch (therefore it has three terminals), which is made by Omron Electronics [92] was utilized. The concept is that the switch will be activated by newly developed cold-reactive LCEs. Therefore, the sensor's operation is purely passive, temperature dependent, and reversibly functional. However, this switch has been designed to operate at DC. Consequently, a model of the switch's RF performance must be developed in the RFID UHF band, which must be 
included in the simulation analysis. The procedure for deriving this model is discussed in what follows.

Initially, a de-embedding circuit is fabricated. The circuit consists of the switch with each terminal connected to a transmission line, which is terminated to an SMA port, as shown in Figure 7.6(a). Additionally, an identical circuit is made without the switch to compensate for the electrical length of the transmission lines (i.e., set the reference planes exactly at the terminals of the switch), as shown in Figure 7.6(b). The electrical lengths of transmission lines are calculated by using the open-circuit auto port-extension feature of the E5061B Vector Network Analyzer (VNA).

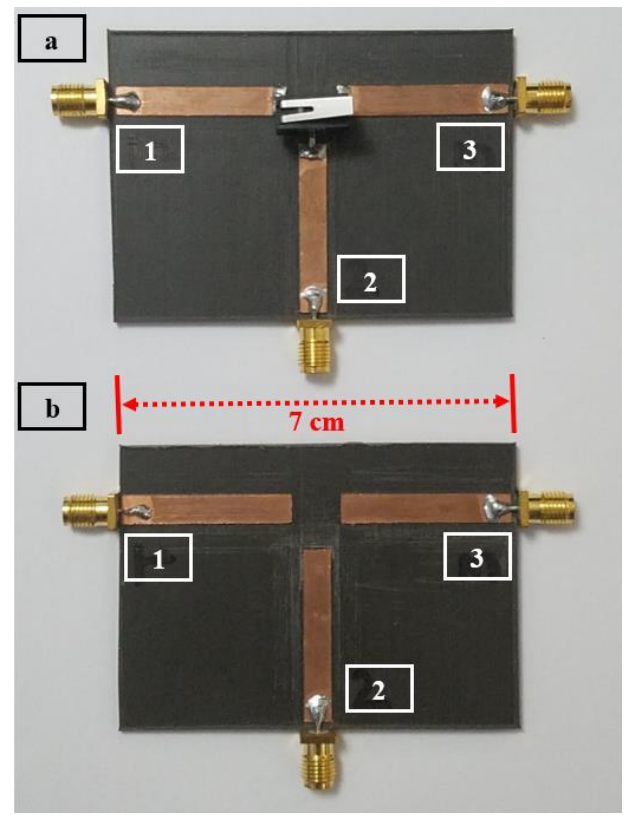

Figure 7.6. Mechanical switch testing circuit. (a) Mechanical switch with deembedding circuit. (b) De-embedding circuit for electrical length compensation.

Since the switch is passive, it has $S$-parameters that are reciprocal, i.e., $S_{i j}=S_{j i}$, [40, p. 175]. Using the design in Figure 7.6(b), an open-circuit auto port-extension is conducted while connected to two ports; for example, ports 1 and 2. Subsequently, this circuit is disconnected and the circuit from Figure 7.6(a) is connected to the corresponding 
ports (i.e., ports 1 and 2). The third remaining port is terminated into a $50 \Omega$ load. The 2 port S-parameters are then measured. This process is repeated for ports 1 and 3 , and ports 2 and 3. These three 2-port measurement sets are then combined to form a single 3-port touchstone file. This file is used as the switch's model and it is imported in ANSYS Circuit Designer. Notably, the three measurements mentioned above are conducted for both activated (ON) and de-activated (OFF) states of the switch. The switch's insertion losses are plotted in Figure 7.7 for both states.

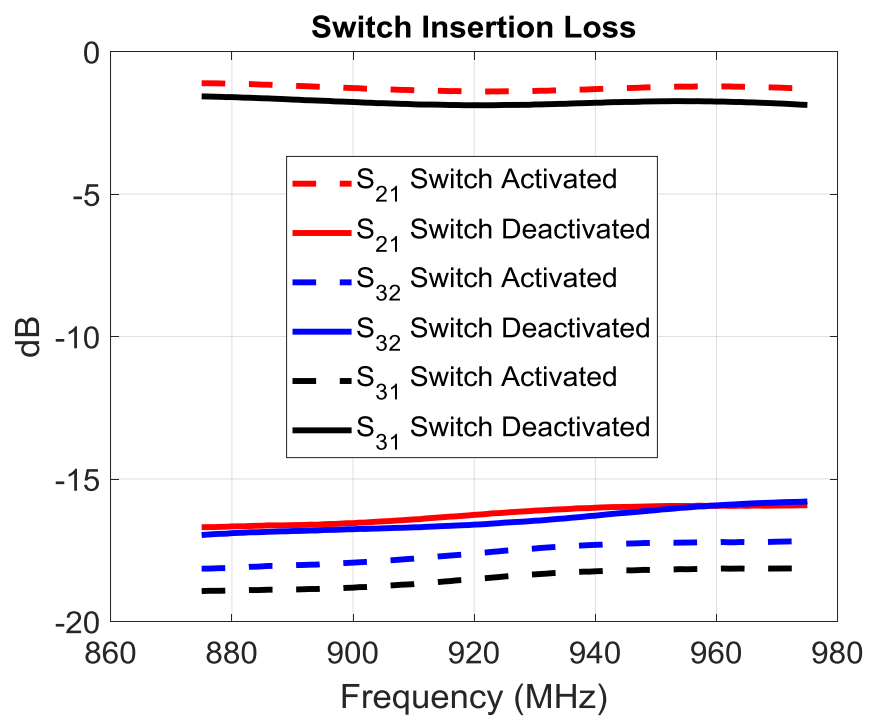

Figure 7.7. Insertion losses of the 3-port mechanical switch for the deactivated and activated states.

\subsection{Matching Network Design}

According to the sensor's design (refer Figure 7.1) an RFID IC needs to be connected to each of the terminals of the switch. However, before placing each IC, an appropriate matching network must be designed. The objective is to design a pair of matching networks (at the output of each switch terminal) that will maximize the power output to one IC (when the switch is activated) and minimize the power output to the other 
IC. Additionally, when the switch is deactivated, the matching networks should operate to match the ICs in the reverse fashion.

The antenna's simulation results agree well with the measured ones, as shown in Figure 7.5. Therefore, the simulated data for the antenna is used for the sensor's system modeling and design. Specifically, the antenna's simulated 1-port model along with the measured 3-port model of the switch (for the case when the switch is activated and when it is deactivated) are exported and connected in ANSYS Circuit Designer. The remaining terminals of the switch are connected to $50 \Omega$ ports (i.e., ports A and B) as shown in Figure 7.8.

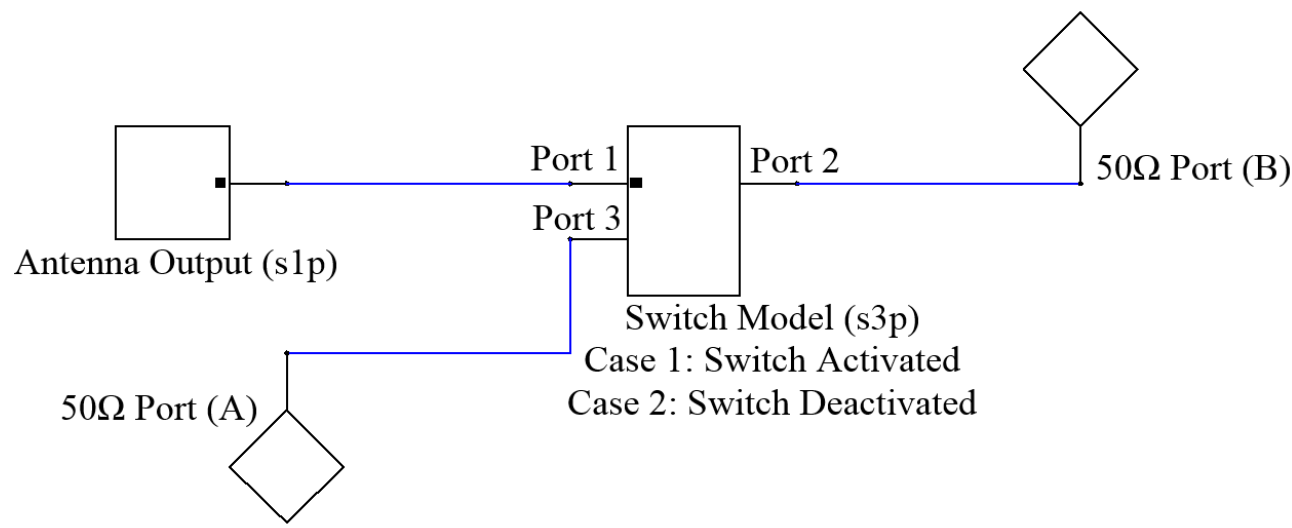

Figure 7.8. Antenna and mechanical switch model in ANSYS Circuit Designer. Using this model in ANSYS Circuit Designer, the impedances (with the antenna and switch) at ports 2 and 3 can be found for both switch states (i.e., activated and deactivated). This provides the needed information for designing the corresponding matching networks.

Firstly, the impedance at port 2 is measured for when the switch is activated. This impedance is then matched to the conjugate of the RFID IC 2 using a matching network. When the switch is deactivated, the impedance at port 2 will significantly change thereby deteriorating the matching. Therefore, no power will reach RFID IC 2 when the switch is 
in the deactivated state and will remain OFF (as intended). The impedances at port 2 for both activated and deactivated cases are shown in Figure 7.9, and they are indeed very different.

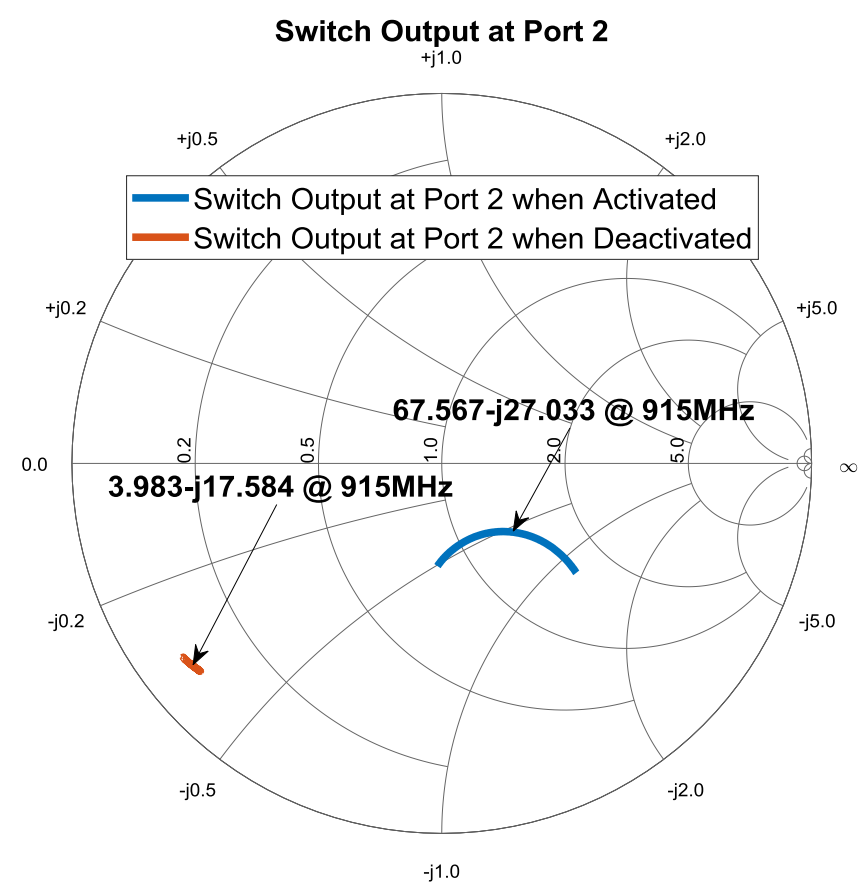

Figure 7.9. Input impedance at port 2 for switch in activated and deactivated states.

In the deactivated case, we have indicated that little power will be transferred to the IC at port 2; however, at port 3 we seek to maximize the power transfer. The impedance at port 3 is measured for when the switch is deactivated using the circuit model shown in Figure 7.8. This impedance is then matched to the conjugate of the RFID IC 1 using a matching network. When the switch is activated, the impedance at port 3 will significantly change thereby deteriorating the matching. Therefore, no power will reach RFID IC 1 when the switch is in the activated state and will remain OFF (as intended). The impedances at port 3 for both activated and deactivated cases are shown in Figure 7.10, and they are indeed very different. 


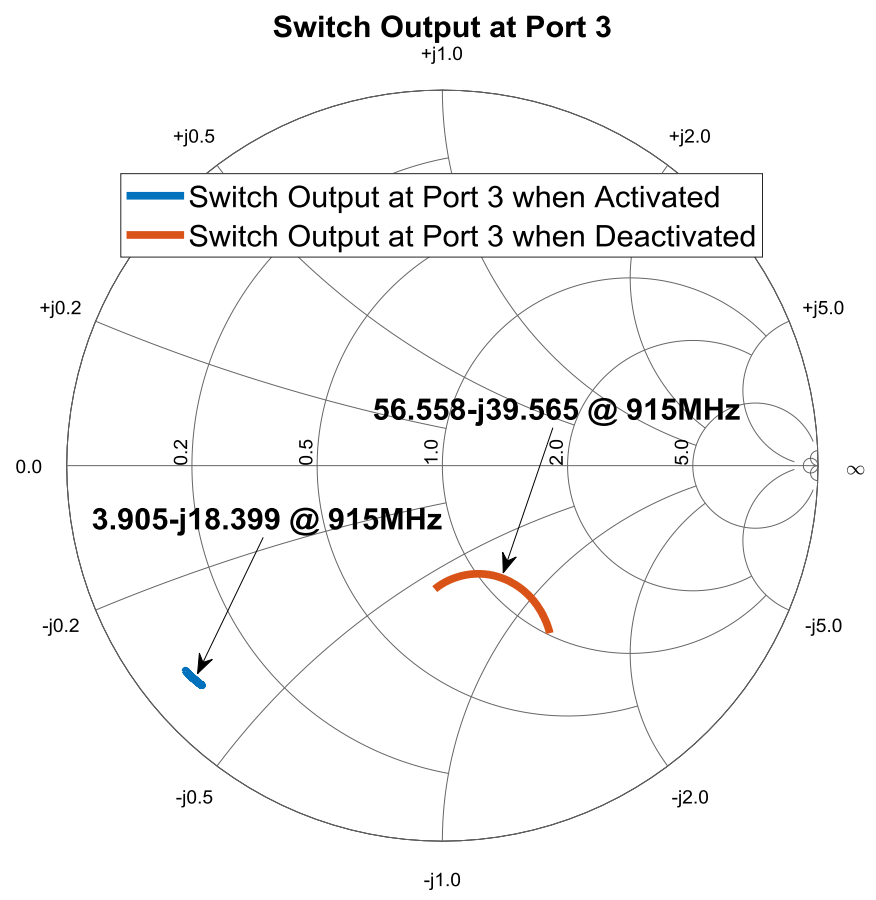

Figure 7.10. Input impedance at port 3 for switch in activated and deactivated states.

The final design layout with the antenna, switch, and designed matching networks are depicted in Figure 7.11.

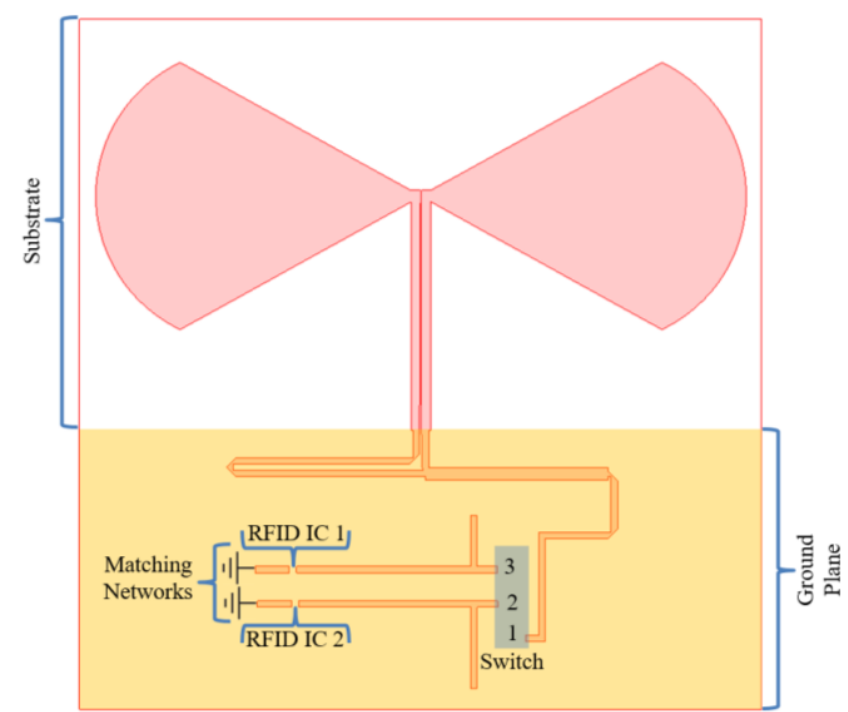

Figure 7.11. RFID sensor design layout.

Furthermore, to test the degree of matching between each switch port and the RFID IC, we utilize the power transmission coefficient (PTC or $\tau ; 0 \leq \tau \leq 1$ ). When $\tau=1$, a 
conjugate match $\left(\mathrm{Z}_{\text {port }}=\mathrm{Z}_{\mathrm{IC}}^{*}\right)$ is obtained that provides maximum power transfer to the IC as discussed in Section 2.6.

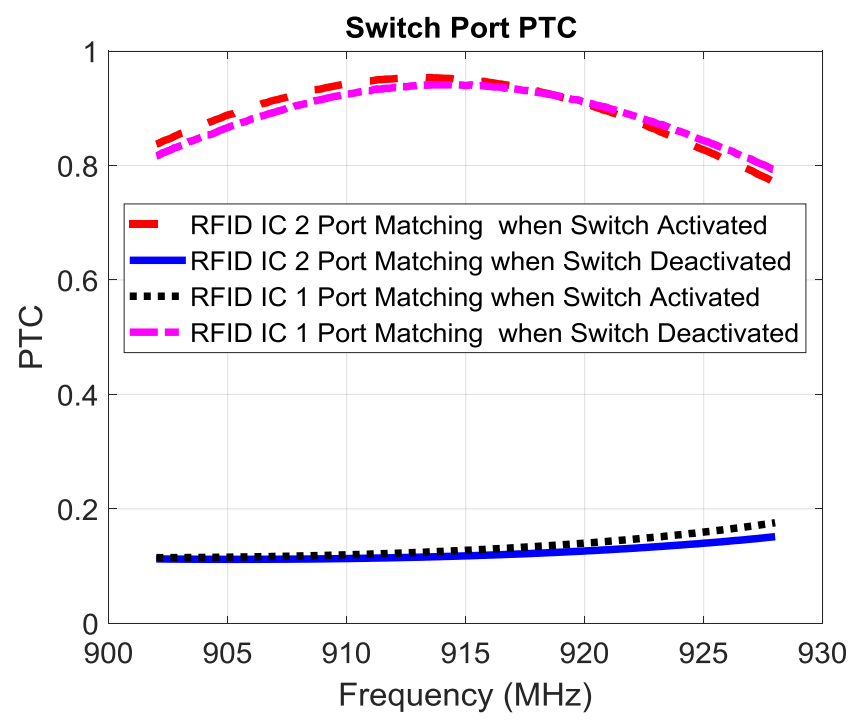

Figure 7.12. Simulated PTC at the ports where RFID ICs 1 and 2 will be connected, as shown in Figure 7.11.

Figure 7.12 shows the PTC for both switch states at the two ports, where the RFID ICs 1 and 2 will be connected as per Figure 7.11. These results clearly illustrate the following: (a) when the switch is activated, RFID IC 2 (at port 2 of the switch, refer to Figure 7.8) will be ON and RFID IC 1 (at port 3 of the switch, refer to Figure 7.8) will be OFF, and (b) when the switch is deactivated, RFID IC 2 (at port 2 of the switch, refer to Figure 7.8) will be OFF and RFID IC 1 (at port 3 of the switch, refer to Figure 7.8) will be ON.

\subsection{Measurements of Switching Capability}

Initially, a prototype of the sensor antenna is fabricated. Subsequently, it is measured using a VNA to validate the sensor's capability to switch the power flow from one RFID IC to the other, as predicted by the simulation results in Figure 7.12. The measurements are conducted as follows: the input impedance at the RFID IC 1 port is 
measured while the RFID IC 2 port is left open (see Figure 7.11). The RFID IC 2 port can be left open because the isolation between the two ports is significant (i.e., >18 dB) irrespective of the switch activation status, as shown in Figure 7.13.

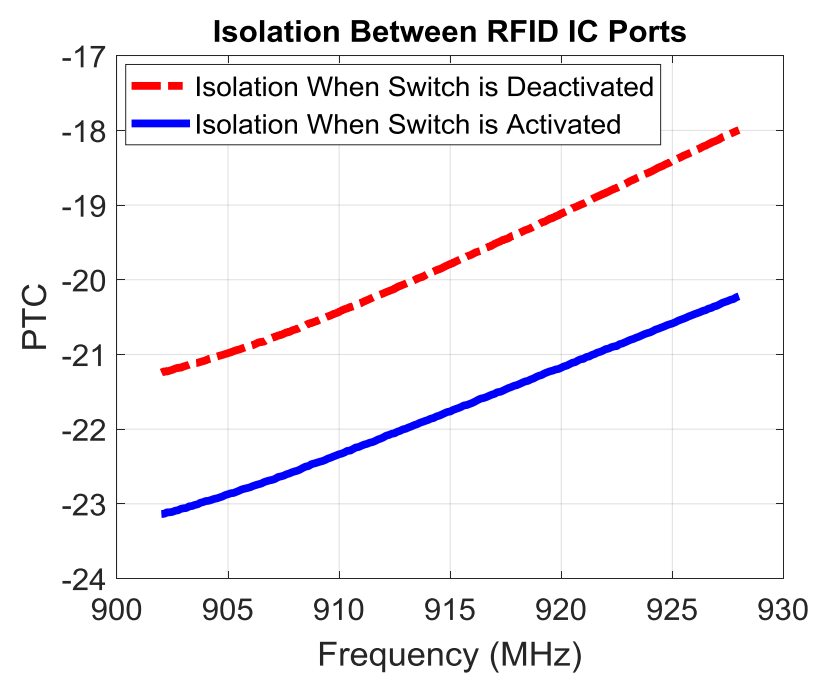

Figure 7.13. Isolation between RFID IC 1 port and RFID IC 2 port.

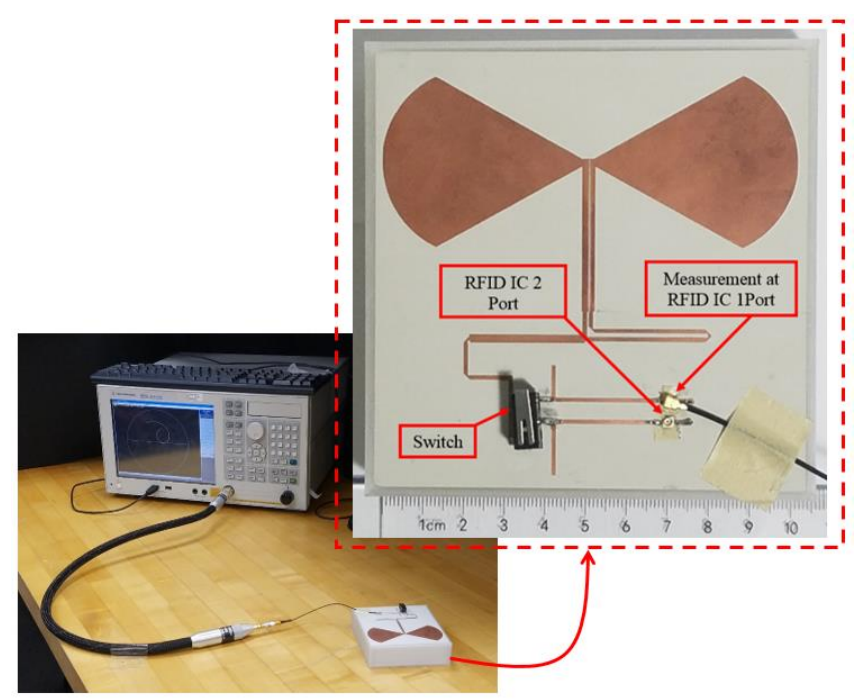

Figure 7.14. Test setup with fabricated prototype for impedance measurements at the ports where the RFID ICs are connected.

Figure 7.14 shows the test setup and the fabricated prototype. An IPX interface is used to measure the impedance of RFID IC 1 port for both switch states (i.e., activated and 
deactivated) while RFID IC 2 port is open. These measurements are depicted in Figure 7.15 .

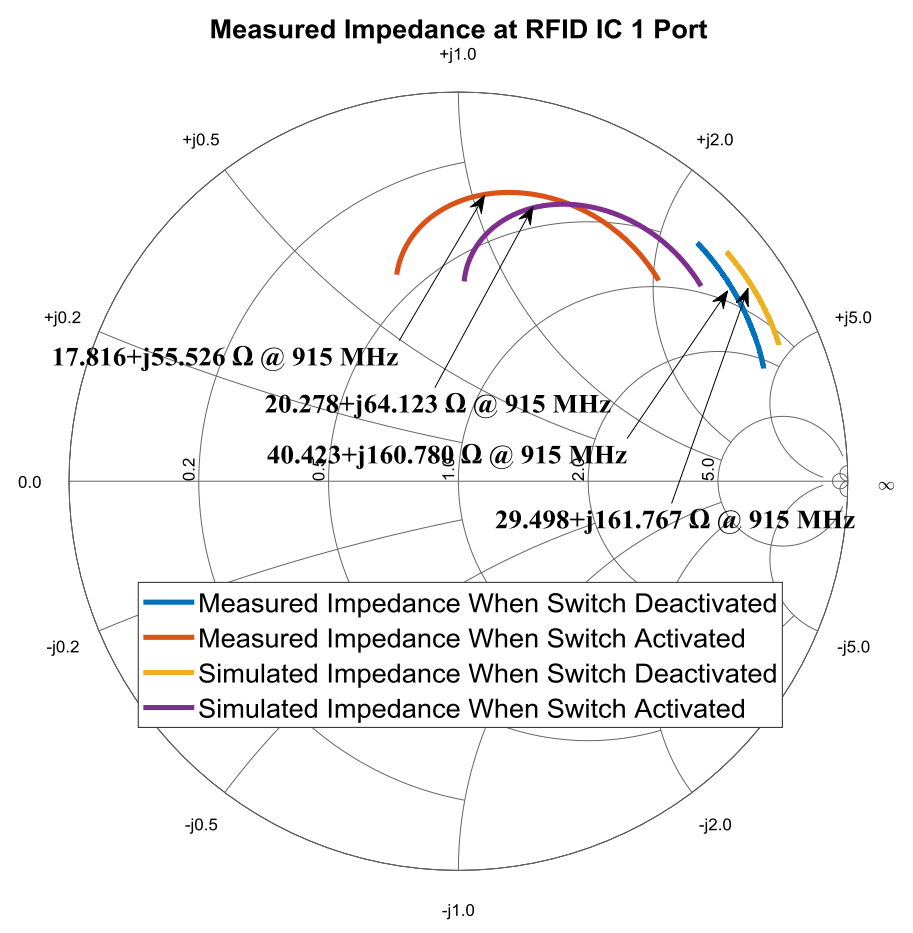

Figure 7.15. Simulated and measured input impedances at RFID IC 1 port. 


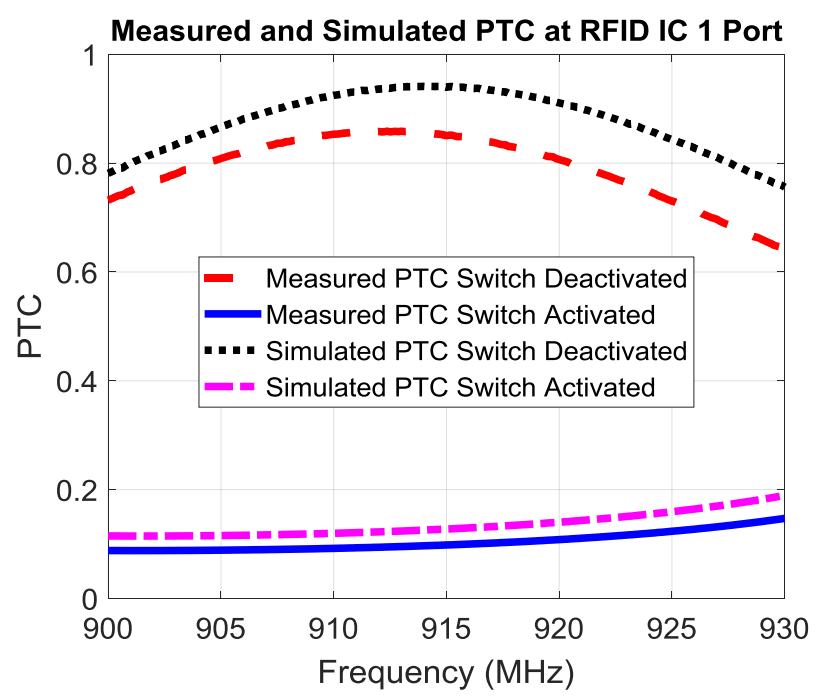

Figure 7.16. Simulated and measured PTC at RFID IC port 1.

Moreover, the corresponding PTCs are plotted in Figure 7.16. According to Figures 7.15 and 7.16, the measured and simulated impedance at RFID IC 1 port agree very well. These results indicate good matching when the switch is deactivated and the intentional degradation in the matching when the switch is activated. Also, Figure 7.16 shows that when the switch is activated and deactivated, the power flow to RFID IC 1 is minimized and maximized, respectively.

To further examine the power-flow switching capability of the design, the same test is conducted for RFID IC 2 port. Namely, the measurements are performed at RFID IC 2 port with RFID IC 1 port open. The simulated and measured results for this case are depicted in Figure 7.17. 


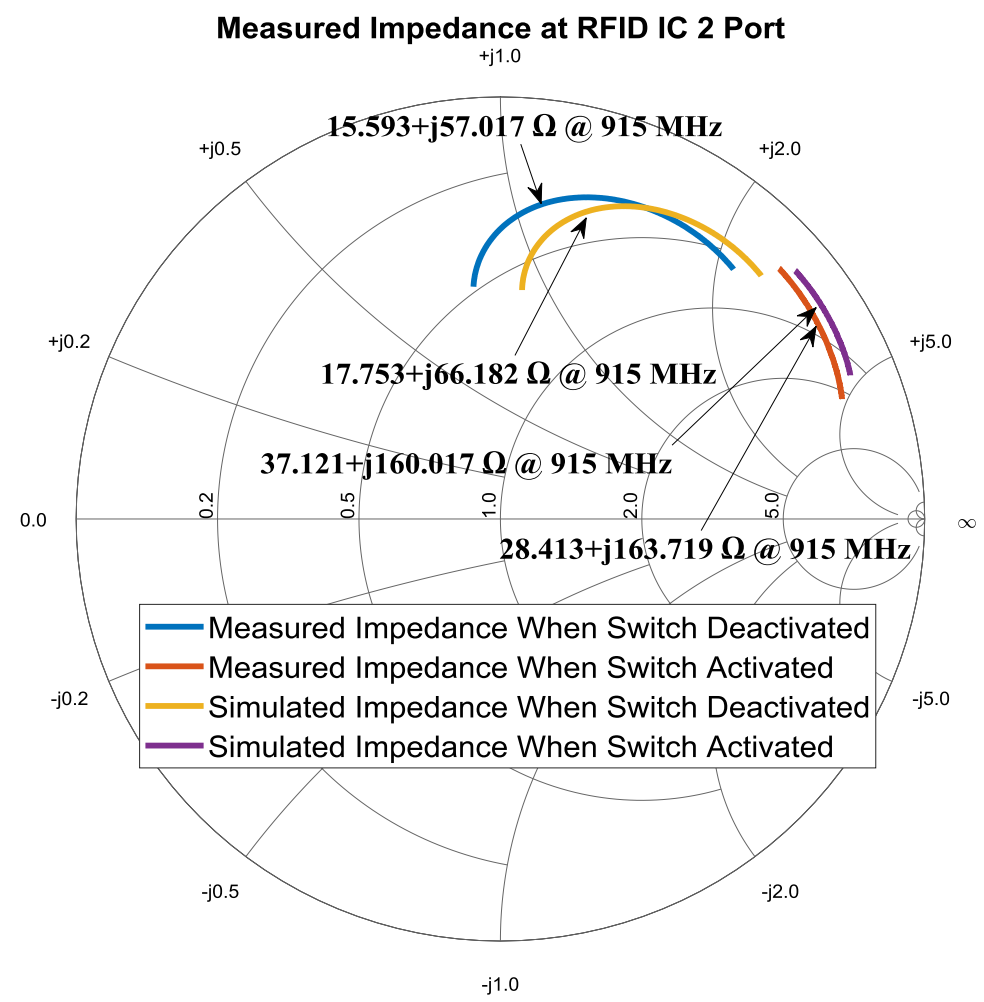

Figure 7.17. Simulated and measured PTC at RFID IC port 2.

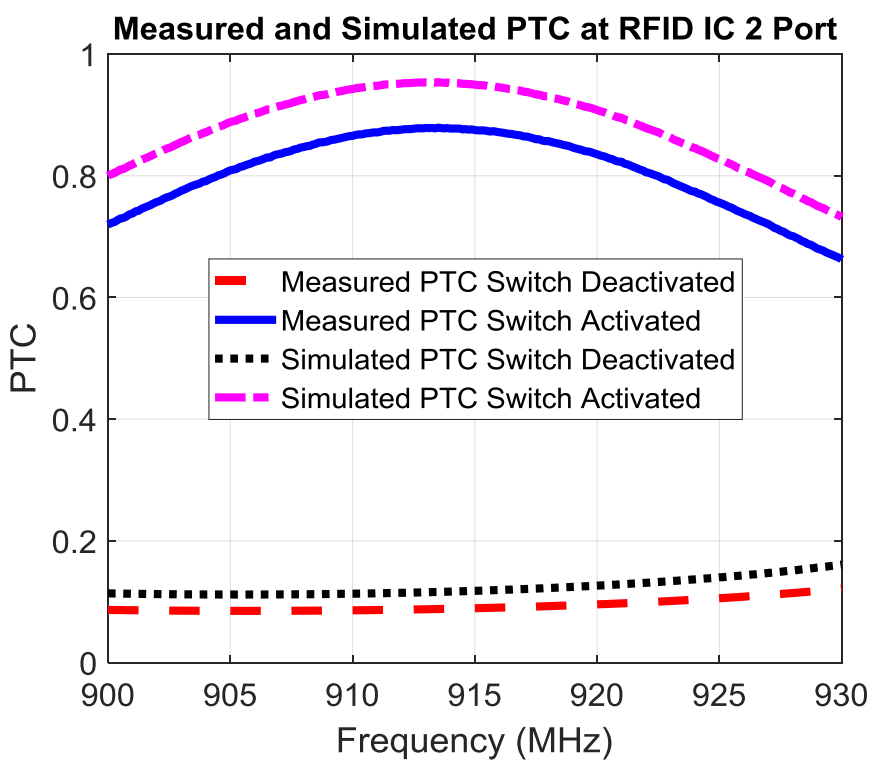

Figure 7.18. Simulated and measured PTC at RFID IC port 2.

Furthermore, the corresponding PTCs are plotted in Figure 7.18. It is clearly seen from these results that the measurements agree well with simulations and confirm the intended 
operation of the design. Specifically, when the switch is deactivated and activated, the power flow to RFID IC 2 is minimized and maximized, respectively.

\subsection{Validation of Temperature Sensor}

The final design of the sensor is depicted in Figure 7.19.

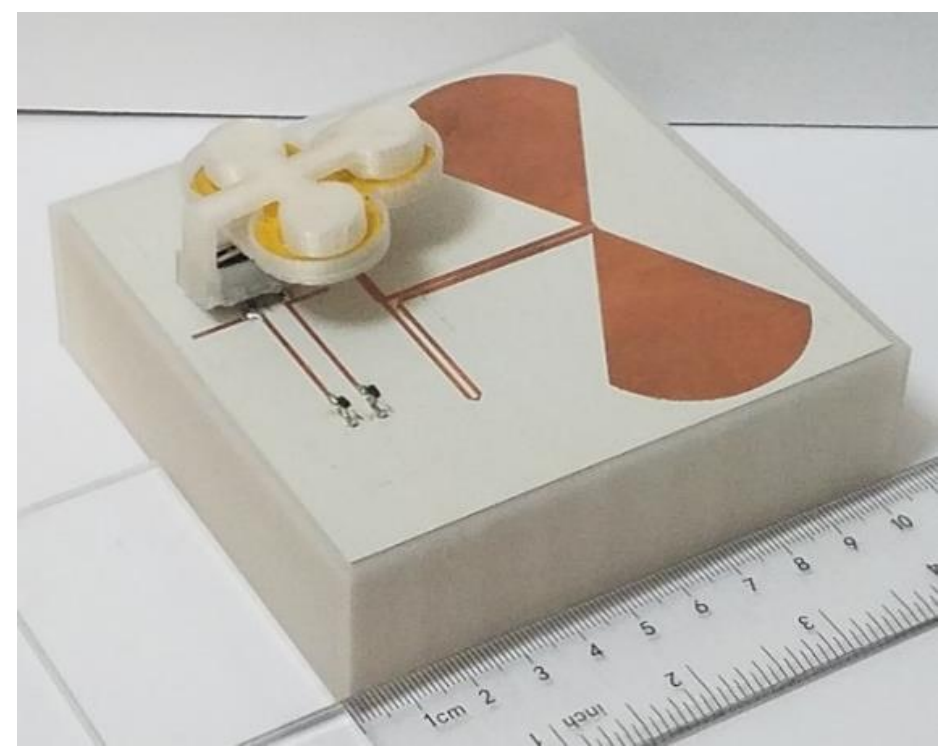

Figure 7.19. RFID temperature sensor with cold-responsive LCE array.

The mechanical switch has been fitted with a customized housing consisting of two parts. The first part is a stationary housing, which is anchored to the base of the switch. The second part consists of a platform to support the LCE array and is situated on the lever of the switch. When the environmental temperature is lower or equal than approximately $-10^{\circ}$ C, the LCE is activated (i.e., cold-responsive LCE) and it pushes against the stationary housing and triggers the switch. When the environmental temperature rises and becomes larger than approximately $-10^{\circ} \mathrm{C}$, the LCE returns to its relaxed state and the switch returns to its original position on its own. 
The temperature sensing ability of the RFID passive sensor is tested using the ThingMagic RFID reader as detailed here. The test setup is depicted in Figure 7.20.

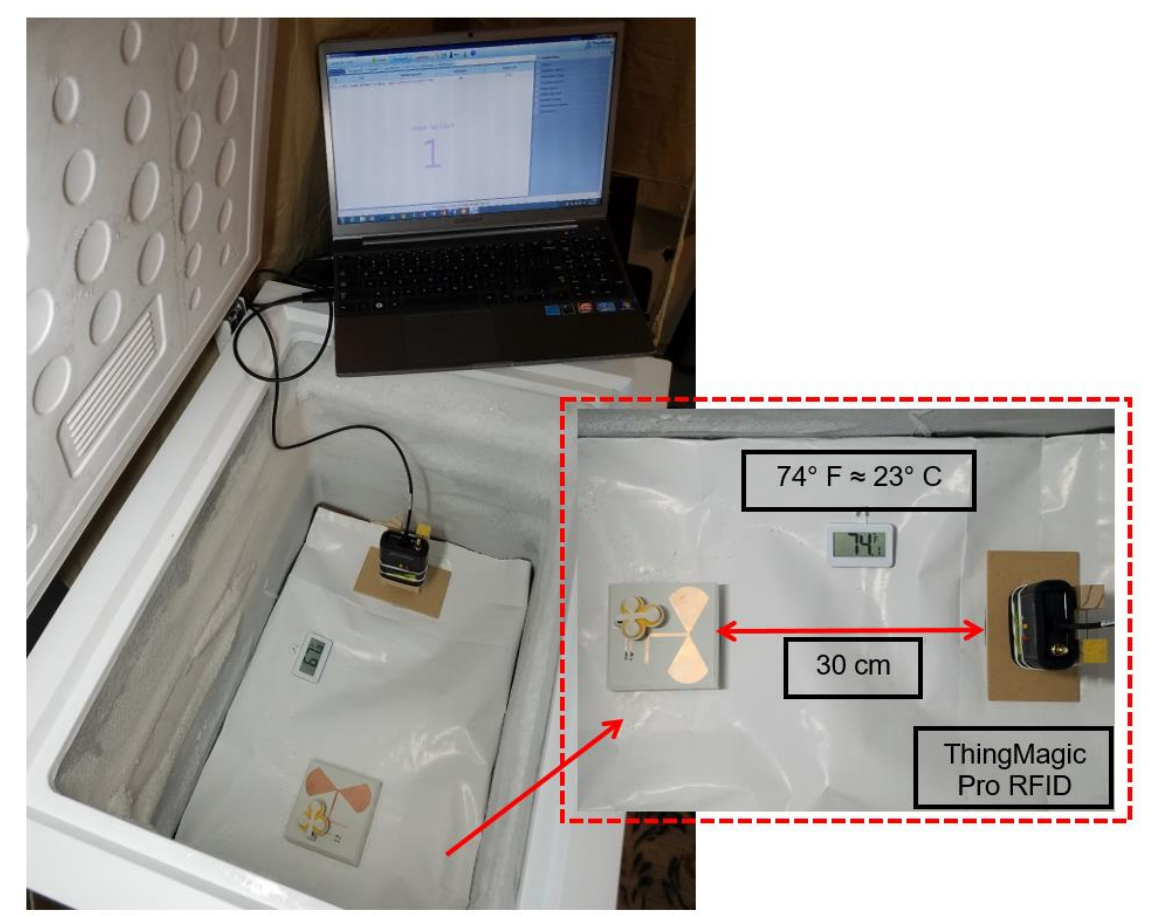

Figure 7.20. RFID temperature sensor with RFID reader inside freezer at roomtemperature.

To distinguish which RFID ICs are read during the different stages of the test, the "Tag Aging" feature is activated in the RFID reader's software. This feature highlights in gray any unreadable tags within the read range of the reader. Initially, the sensor is placed inside a non-operating freezer. Therefore, the inside volume of the freezer is at room temperature (i.e., $23^{\circ} \mathrm{C}$ ). Since the cold-responsive LCE is not activated at such temperatures, the switch is also not activated and the RFID reader senses only the ID of RFID IC 1, as shown in Figure 7.21. 


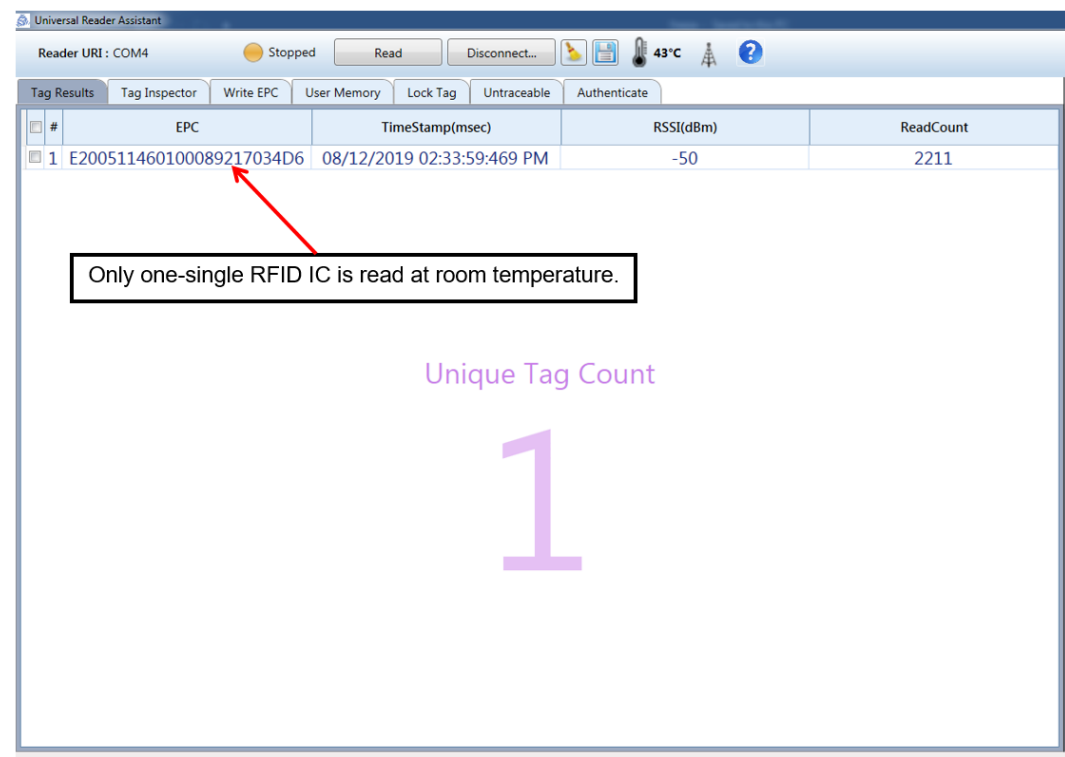

O Thing Magic, a Division of Trimble. All Rights Reserved.

Figure 7.21. Only RFID IC 1 is read at room temperature.

Subsequently, the RFID reader is removed from the freezer while the sensor stays inside the freezer. Then, the freezer is shut and turned on. We allow the freezer to cool for 30 min to a temperature of approximately $-15^{\circ} \mathrm{C}$. Then, the RFID reader is reinserted into the freezer as shown in Figure 7.22.

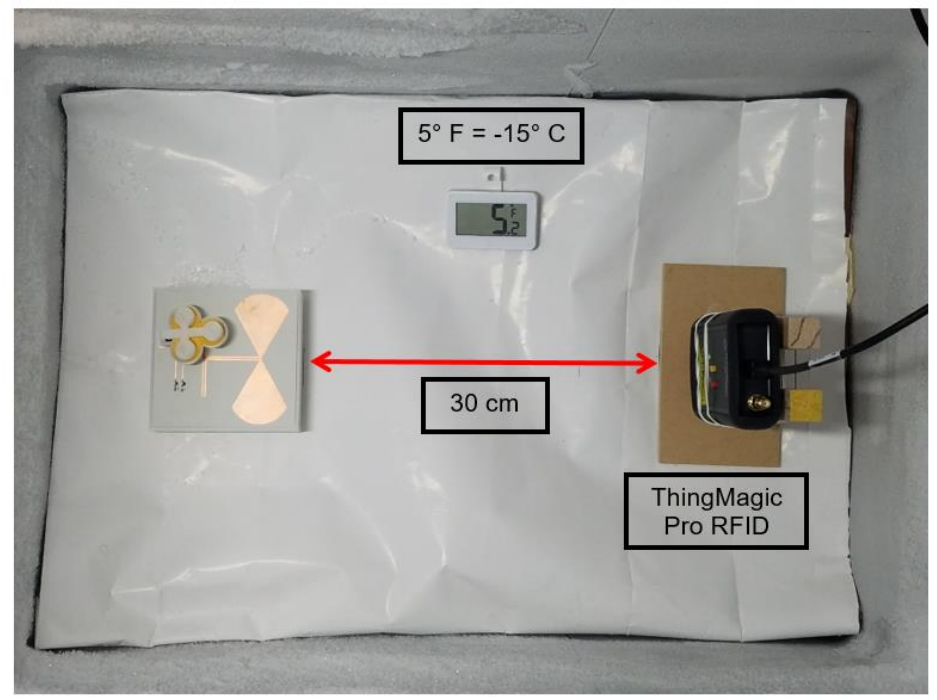

Figure 7.22. RFID temperature sensor with RFID reader inside freezer at $-15^{\circ} \mathrm{C}$.

In this case, we see that due to the cold temperature the cold-responsive LCE has activated the switch and only the ID of RFID IC 2 is read, as shown in Figure 7.23. 


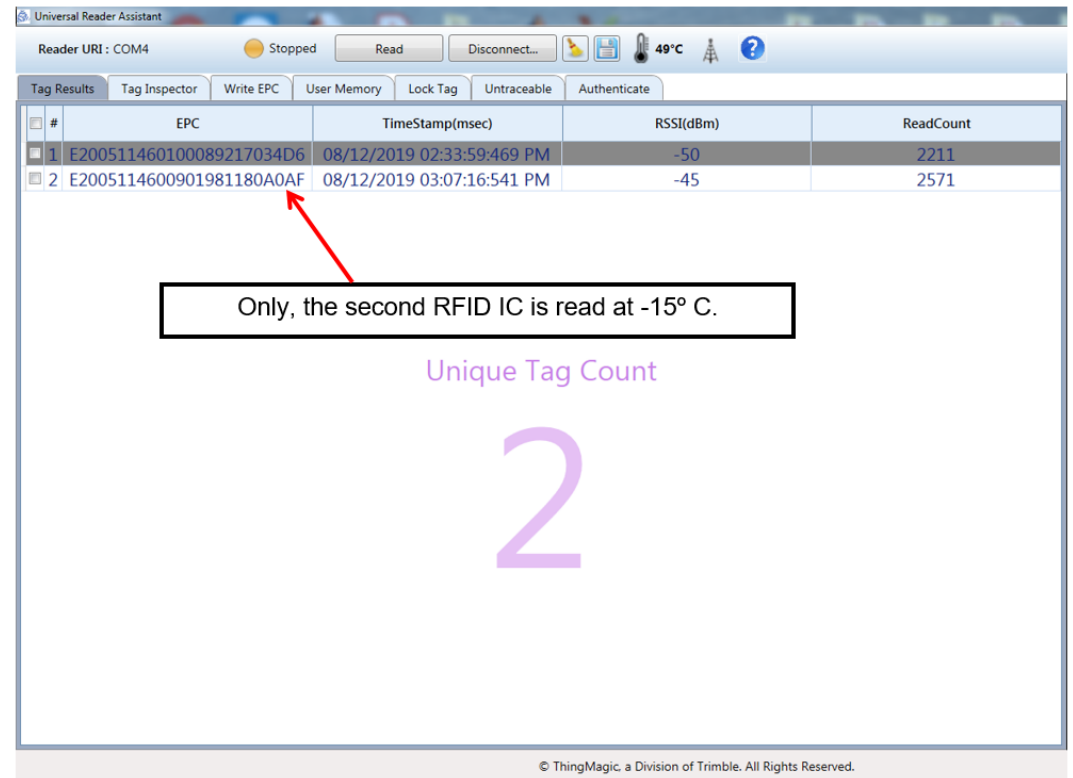

Figure 7.23. Only RFID IC 2 is read at $-15^{\circ} \mathrm{C}$.

The ID of RFID IC 1 is clearly highlighted in gray, which means it is not detected by the reader. Moreover, the software also shows that two unique tags were detected (with two different EPCs) within the total time-duration of the test. Figures 21 and 23 are also timestamped to demonstrate the consistency in the experiment. Finally, once the freezer is turned off and the temperature returns to room temperature (i.e., $23^{\circ} \mathrm{C}$ ), the LCE returns to its relaxed state and the switch is deactivated. In this case, the RFID reader returns to reading only RFID IC 1 as shown in Figure 21. Therefore, the experiment clearly proves that the passive RFID sensor is a not a single-use sensor. In fact, this sensor provides continuous and independent temperature monitoring and detects multiple temperature threshold crossings from cold to room temperature and vice versa.

\subsection{Conclusion}

In this work, a sensor, which conveys changes in temperature by switching its operation between two unique RFID ICs, is developed. Therefore, this sensor detects 
temperature threshold crossing in a very clear and robust manner, i.e., at cold temperature one RFID IC ID is read and at room temperature another RFID IC ID is read. Our sensor uses novel cold-reactive liquid crystal elastomers that operate reversibly. Hence, this sensor can autonomously operate through multiple room-to-cold and cold-to-room temperature cycles. Also, this sensor has a battery-free operation (i.e., it is purely passive).

The design of this sensor consists of a wideband bow-tie antenna, which operates in the UHF RFID band of 902-928 MHz. This antenna was fed with a CPS to microstrip transmission line conversion. A balun was also designed using a pair of feed-lines $180^{\circ}$ out of phase, which are matched to $50 \Omega$ using a quarter-wave transformer. The input of the antenna is connected to a mechanical switch, which swaps the operation of the tag between two RFID ICs based on the temperature and by using novel cold-responsive LCEs. 


\section{CHAPTER 8}

\section{CONCLUSION AND FUTURE WORK}

\subsection{Conclusions}

This dissertation presents the design, fabrication and testing of novel temperature sensors that are applicable to the Cold Supply Chain. These sensors are unique as they incorporate novel shape-shifting Liquid Crystal Elastomers (LCEs), which are programmable and reversible. The goal in this research was to design antennas and corresponding feed structures that integrate LCEs and translate their thermal dependent actuation into controlled and detectable changes in antenna performance. Additionally, the RFID platform was utilized due to its established communication protocol.

As a result, three designs were created and are presented in this research. The first two designs convey temperature threshold crossings in the frequency domain and the third one conveys temperature threshold crossings by switching between two individual RFID ICs, where each IC has a unique code. Each design performed successfully and conveyed a distinct frequency shift or IC EPC change. Moreover, all designs fulfilled the research objectives that were to: (a) operate through purely passive technology (i.e., battery-free sensing) in order to ensure operational longevity and cost-effectiveness, (b) monitor individual items or crates (i.e., operate wirelessly), (c) function in a continuous temperature monitoring mode (e.g., able to detect multiple room-to-cold and cold-to-room temperature cycles), (d) operate without requiring any form of resetting (i.e., operate autonomously), and (e) have a compact form-factor to fit on items of various sizes in the CSC. 


\subsection{Future Work}

Experimentation with flexible substrates is important to accommodate packages of irregular shapes and sizes. Furthermore, printed antennas on full LCE substrates may be a new gateway for compact and cost-effective sensor systems. Also, reconfigurable RF components, such as, waveguides, filters and couplers, can incorporate LCEs to provide novel sensor designs. Additionally, the development of simple and cost-effective RFID temperature sensors, which stay $\mathrm{ON}$ at certain temperatures while they detune and turn OFF at certain temperature-thresholds, could be explored using antennas with LCEs. Finally, LCE technologies that respond to IR light and (hot or cold) water could be utilized in new sensors for various applications. 


\section{REFERENCES}

[1] "The Cold Chain and its Logistics," The Geography of Transport Systems, 13-Oct2019. [Online]. Available: https://transportgeography.org/?page_id=6585. [Accessed: 28-Dec-2019].

[2] Dada, A. and Thiesse, F. (2018). Sensor Applications in the Supply Chain: The Example of Quality-Based Issuing of Perishables. [online] Available at: https://rd.springer.com/content/pdf/10.1007\%2F978-3-540-78731-0_9.pdf [Accessed 14 May 2018].

[3] R. Bhattacharyya, C. Floerkemeier, S. Sarma and D. Deavours, "RFID tag antenna based temperature sensing in the frequency domain," 2011 IEEE International Conference on RFID, Orlando, FL, 2011, pp. 70-77.

[4] United World Transportation. (2018). The 4 Most Common Cold Chain Logistics Problems Truckers Face and Tips for Solving Them. [online] Available at: https://unitedworldtransportation.com/4-common-cold-chain-logistics-problemstruckers-face-tips-solving/ [Accessed 6 May 2018].

[5] Facts, U. (2018). Topic: Organic Food Industry. [online] www.statista.com. Available at: https://www.statista.com/topics/1047/organic-food-industry/ [Accessed 14 May 2018].

[6] Finkenzeller, K. RFID Handbook: Fundamentals and Applications in Contactless Smart Cards, Radio Frequency Identification and near-Field Communication. 2nd ed. Chichester: John Wiley \& Sons, Ltd, 2003.

[7] D. Dobkin, The RF in RFID, 1st ed. Amsterdam: Elsevier, 2008.

[8] J. Virtanen, L. Ukkonen, T. Björninen, L. Sydänheimo and A. Z. Elsherbeni, "Temperature sensor tag for passive UHF RFID systems," 2011 IEEE Sensors Applications Symposium, San Antonio, TX, 2011, pp. 312-317.

[9] C. P. Ambulo, J. J. Burroughs, J. M. Boothby, H. Kim, M. R. Shankar, and T. H. Ware, "Four-dimensional Printing of Liquid Crystal Elastomers," ACS Applied Materials \& Interfaces, vol. 9, no. 42, pp. 37332-37339, Nov. 2017.

[10] Küpfer, J. and Finkelmann, H. (1991). Nematic liquid single crystal elastomers. [online] Wiley Online Library. Available at: https://onlinelibrary.wiley.com/doi/pdf/10.1002/marc.1991.030121211 [Accessed 24 Aug. 2018]. 
[11] Kularatne, R., Kim, H., Boothby, J. and Ware, T. (2017). Liquid crystal elastomer actuators: Synthesis, alignment, and applications. [online] Wiley Online Library. Available at: https://doi.org/10.1002/polb.24287 [Accessed 24 Aug. 2018].

[12] J. S. Gibson, X. Liu, S. V. Georgakopoulos, J. J. Wie, T. H. Ware and T. J. White, "Reconfigurable Antennas Based on Self-Morphing Liquid Crystalline Elastomers," in IEEE Access, vol. 4, pp. 2340-2348, 2016.

[13] Y. Shafiq, J. S. Gibson, H. Kim, C. P. Ambulo, T. H. Ware and S. V. Georgakopoulos, "A Reusable Battery-Free RFID Temperature Sensor," in IEEE Transactions on Antennas and Propagation, vol. 67, no. 10, pp. 6612-6626, Oct. 2019.

[14] C. Liu, H. Qin and P. Mather, "Review of progress in shape-memory polymers", Journal of Materials Chemistry, vol. 17, no. 16, p. 1543, 2007. Available: 10.1039/b615954k [Accessed 24 January 2019].

[15] P. Kumar, H. Reinitz, J. Simunovic, K. Sandeep and P. Franzon, "Overview of RFID Technology and Its Applications in the Food Industry", Journal of Food Science, vol. 74, no. 8, pp. R101-R106, 2009.

[16] K. Biji, C. Ravishankar, C. Mohan and T. Srinivasa Gopal, "Smart packaging systems for food applications: a review", Journal of Food Science and Technology, vol. 52, no. 10, pp. 6125-6135, 2018.

[17] Ams.usda.gov. (2008). Protecting Perishable Foods During Transport by Truck. [online] Available at: https://www.ams.usda.gov/sites/default/files/media/TransportPerishableFoodsbyTr uck\%5B1\%5D.pdf [Accessed 5 May 2018].

[18] Temptrust.mesalabs.com. (2001). Recommendations on the control and monitoring of storage and transportation temperatures of medicinal products. [online] Available at: https://temptrust.mesalabs.com/wpcontent/uploads/sites/47/2012/05/MHRAcon007569.pdf [Accessed 5 May 2018].

[19] "Cold Chain - Why Is It Growing So Fast?," The Logistics of Logistics, 23-Mar2018. [Online]. Available: https://www.thelogisticsoflogistics.com/cold-chaingrowing-fast/. [Accessed: 20-Oct-2018].

[20] Trade.gov. (2016). 2016 Top Markets Report Cold Supply Chain. [online] Available at: https://www.trade.gov/topmarkets/pdf/Cold_Chain_Top_Markets_Report.pdf [Accessed 28 Oct. 2019].

[21] United World Transportation. (2018). The 4 Most Common Cold Chain Logistics Problems Truckers Face and Tips for Solving Them. [online] Available at: 
https://unitedworldtransportation.com/4-common-cold-chain-logistics-problemstruckers-face-tips-solving/ [Accessed 6 May 2018].

[22] Rfidjournal.com. (2019). Georgia Tech. [online] Available at: https://www.rfidjournal.com/articles/pdf?4760 [Accessed 7 Dec. 2019].

[23] Foster, K. and Jaeger, J. (2019). RFID Inside: The murky ethics of implanted chips. [online] Repository.upenn.edu. Available at: https://repository.upenn.edu/cgi/viewcontent.cgi?article=1098\&context=be_papers [Accessed 7 Dec. 2019].

[24] Brandl, Martin \& Kellner, Karl-Heinz \& Grabner, G \& Grabner, J. (2010). Utilizing RFID Technology for Biomedical Sensor Applications. IFMBE Proceedings. 25. 131-133.

[25] C. Occhiuzzi, S. Caizzone and G. Marrocco, "Passive UHF RFID antennas for sensing applications: Principles, methods, and classifcations," in IEEE Antennas and Propagation Magazine, vol. 55, no. 6, pp. 14-34, Dec. 2013.

[26] C. Occhiuzzi and G. Marrocco, "Constrained-Design of Passive UHF RFID Sensor Antennas," in IEEE Transactions on Antennas and Propagation, vol. 61, no. 6, pp. 2972-2980, June 2013.

[27] G. Marrocco, "Pervasive electromagnetics: sensing paradigms by passive RFID technology," in IEEE Wireless Communications, vol. 17, no. 6, pp. 10-17, December 2010.

[28] P. Kumar, H. Reinitz, J. Simunovic, K. Sandeep and P. Franzon, "Overview of RFID Technology and Its Applications in the Food Industry", Journal of Food Science, vol. 74, no. 8, pp. R101-R106, 2009.

[29] Smartrac-group.com. (2019). SENSOR TEMPERATURE DOGBONE - RFID Smartrac. [online] Available at: https://www.smartrac-group.com/sensortemperature-dogbone.html [Accessed 23 Jul. 2019].

[30] Infratab Inc. (2018). Freshtime tags. [online] Available at: http://infratab.com/freshtime-tags/ [Accessed 7 Jul. 2018].

[31] Rfidjournal.net. (2018). [online] Available at: http://www.rfidjournal.net/masterPresentations/live2009/np/Finalist_IDS_Microsh ip.pdf [Accessed 7 Jul. 2018].

[32] Finkenzeller, K. RFID Handbook: Fundamentals and Applications in Contactless Smart Cards, Radio Frequency Identification and near-Field Communication. 2nd ed. Chichester: John Wiley \& Sons, Ltd, 2003. 
[33] C. A. Balanis, Antenna theory analysis and design, 3rd ed. Hoboken, NJ: Wiley, 2005.

[34] J. D. Kraus, Antennas, 2nd ed. New York: McGraw-Hill, 1988.

[35] Alientechnology.com. (2019). [online] Available at: http://www.alientechnology.com/wp-content/uploads/ALC-360-SOT\%20Higgs3\%20SOT\%20(2016-11-03).pdf [Accessed 20 Mar. 2018].

[36] T. Bjorninen, M. Lauri, L. Ukkonen, R. Ritala, A. Elsherbeni and L. Sydanheimo, "Wireless Measurement of RFID IC Impedance", IEEE Transactions on Instrumentation and Measurement, vol. 60, no. 9, pp. 3194-3206, 2011.

[37] K. RamaDevi, A. M. Prasad, and A. J. Rani, Design of RFID Tag Antennas at UHF Band with Different Materials, $1^{\text {st }}$ ed. Beau-Bassin, Mauritius: LAMBERT Academic Publishing, 2018, p. 53.

[38] G. González, Microwave transistor amplifiers, 2nd ed. Upper Saddle River: Prentice Hall, 1996, p. 47.

[39] P. Nikitin, K. Rao, S. Lam, V. Pillai, R. Martinez and H. Heinrich, "Power reflection coefficient analysis for complex impedances in RFID tag design", IEEE Transactions on Microwave Theory and Techniques, vol. 53, no. 9, pp. 2721-2725, 2005.

[40] D. Pozar, Microwave engineering, 4th ed. Hoboken, NJ: Wiley, 2011.

[41] G. Marrocco, "The art of UHF RFID antenna design: impedance-matching and sizereduction techniques," in IEEE Antennas and Propagation Magazine, vol. 50, no. 1, pp. 66-79, Feb. 2008.

[42] M. Bolic, D. Simplot-Ryl and I. Stojmenović, RFID systems, 1st ed. Hoboken, NJ: Wiley, 2010.

[43] Paul, C. Introduction to electromagnetic compatibility. 2nd ed. Hoboken: John Wiley and Sons, 2006.

[44] N. A. Mohammed, K. R. Demarest and D. D. Deavours, "Analysis and synthesis of UHF RFID antennas using the embedded T-match," 2010 IEEE International Conference on RFID (IEEE RFID 2010), Orlando, FL, 2010, pp. 230-236.

[45] H. -. Son and C. -. Pyo, "Design of RFID tag antennas using an inductively coupled feed," in Electronics Letters, vol. 41, no. 18, pp. 994-996, 1 Sept. 2005. 
[46] Smartrac-group.com. (2019). SENSOR TEMPERATURE DOGBONE - RFID Smartrac. [online] Available at: https://www.smartrac-group.com/sensortemperature-dogbone.html [Accessed 23 Jul. 2019].

[47] Infratab Answering "It is fresh?". (2019). Freshtime ${ }^{\mathrm{TM}}$ Tags. [online] Available at: https://infratab-dev.myshopify.com/collections/freshtime-tags?page=1\# [Accessed 12 Dec. 2019].

[48] Youtube.com. (2019). Infratab Cold Chain Freshness Monitoring System. [online] Available at: https://www.youtube.com/watch?v=5n_XbPunsos [Accessed 12 Dec. 2019].

[49] Rfidjournal.net. (2018). [online] Available at: http://www.rfidjournal.net/masterPresentations/live2009/np/Finalist_IDS_Microsh ip.pdf [Accessed 7 Jul. 2018].

[50] Sembolbarkod.net. (2019). Active UHF Tag i-Q Line. [online] Available at: http://www.sembolbarkod.net/identec/i-Q8\%20English\%20V5.23.pdf [Accessed 13 Dec. 2019].

[51] Fsis.usda.gov. (2019). How Temperatures Affect Food. [online] Available at: https://www.fsis.usda.gov/wps/portal/fsis/topics/food-safety-education/getanswers/food-safety-fact-sheets/safe-food-handling/how-temperatures-affectfood/ct_index [Accessed 13 Dec. 2019].

[52] A. A. Babar, S. Manzari, L. Sydanheimo, A. Z. Elsherbeni and L. Ukkonen, "Passive UHF RFID Tag for Heat Sensing Applications," in IEEE Transactions on Antennas and Propagation, vol. 60, no. 9, pp. 4056-4064, Sept. 2012.

[53] R. Bhattacharyya, C. Floerkemeier, S. Sarma and D. Deavours, "RFID tag antenna based temperature sensing in the frequency domain," 2011 IEEE International Conference on RFID, Orlando, FL, 2011, pp. 70-77.

[54] S. Caizzone, C. Occhiuzzi and G. Marrocco, "Multi-Chip RFID Antenna Integrating Shape-Memory Alloys for Detection of Thermal Thresholds," in IEEE Transactions on Antennas and Propagation, vol. 59, no. 7, pp. 2488-2494, July 2011.

[55] Broer, D., Crawford, G. and Žumer, S. (2011). Cross-linked liquid crystalline systems. Boca Raton: CRC Press.

[56] Kularatne, Ruvini S., Hyun Kim, Jennifer M Boothby, and Taylor H Ware. "Liquid crystal elastomer actuators: Synthesis, alignment, and applications Part B Polymer physics" Journal of polymer science 55, no. 5 (2017): 395-411.

[57] McMurry, J. and Fay, R. (2004). Chemistry. 4th ed. Edimbourg: Pearson, p.1016. 
[58] Warner, Mark, Carl D Modes and Daniel Corbett. "Curvature in nematic elastica responding to light and heat." Proceedings of the Royal Society A: Mathematical, Physical and Engineering Sciences 466 (2010): 2975 - 2989.

[59] J. S. Gibson, X. Liu, S. V. Georgakopoulos, J. J. Wie, T. H. Ware and T. J. White, "Reconfigurable Antennas Based on Self-Morphing Liquid Crystalline Elastomers," in IEEE Access, vol. 4, pp. 2340-2348, 2016.

[60] C. P. Ambulo, J. J. Burroughs, J. M. Boothby, H. Kim, M. R. Shankar, and T. H. Ware, "Four-dimensional Printing of Liquid Crystal Elastomers," ACS Applied Materials \& Interfaces, vol. 9, no. 42, pp. 37332-37339, Nov. 2017.

[61] M. J. Ford, C. P. Ambulo, T. A. Kent, E. J. Markvicka, C. Pan, J. Malen, T. H. Ware, and C. Majidi, "A multifunctional shape-morphing elastomer with liquid metal inclusions," Proceedings of the National Academy of Sciences, vol. 116, no. 43, pp. 21438-21444, Jul. 2019.

[62] López-Valdeolivas, M.; Liu, D.; Broer, D.J.; Sánchez-Somolinos, C., 4D printed actuators with soft-robotic functions. In: Macromolecular Rapid Communications. 2018; Vol. 39, No. 5.

[63] Foffano, G., Lintuvuori, J., Tiribocchi, A. and Marenduzzo, D. (2014). The dynamics of colloidal intrusions in liquid crystals: a simulation perspective. Liquid Crystals Reviews, 2(1), pp.1-27.

[64] T. Ware, "The Ware Research Group," The Ware Research Group, 18-Jun-2019. [Online]. Available: https://warelab.co/. [Accessed: 24-Oct-2019].

[65] T. Ware, D. Simon, K. Hearon, C. Liu, S. Shah, J. Reeder, N. Khodaparast, M. P. Kilgard, D. J. Maitland, R. L. Rennaker, and W. E. Voit, "Three-Dimensional Flexible Electronics Enabled by Shape Memory Polymer Substrates for Responsive Neural Interfaces," Macromolecular Materials and Engineering, vol. 297, no. 12, pp. 1193-1202, 2012.

[66] D. E. Hodgson and M. H. Wu, "Shape Memory Alloys," ASM Handbook, vol. 2, pp. 897-902, 1990.

[67] M. Kubo, X. Li, C. Kim, M. Hashimoto, B. J. Wiley, D. Ham, and G. M. Whitesides, "Stretchable Microfluidic Radiofrequency Antennas," Advanced Materials, vol. 22, no. 25, pp. 2749-2752, 2010.

[68] C. Yu, Z. Duan, P. Yuan, Y. Li, Y. Su, X. Zhang, Y. Pan, L. L. Dai, R. G. Nuzzo, Y. Huang, H. Jiang, and J. A. Rogers, "Electronically Programmable, Reversible Shape Change in Two- and Three-Dimensional Hydrogel Structures," Advanced Materials, vol. 25, no. 11, pp. 1541-1546, 2012. 
[69] S. H. Teoh, "Failure in Biomaterials" in Comprehensive structural integrity: fracture of materials from nano to macro, vol. 9. New York: Elsevier, 2004, p. 163.

[70] Majidi, Leily, Gritsenko, Dmitry, Xu, and Jie, "Gallium-Based Room-Temperature Liquid Metals: Actuation and Manipulation of Droplets and Flows," Frontiers, 07Aug-2017. [Online]. Available: https://www.frontiersin.org/articles/10.3389/fmech.2017.00009/full. [Accessed: 28-Dec-2019].

[71] M. Bahram, N. Mohseni, and M. Moghtader, "An Introduction to Hydrogels and Some Recent Applications," IntechOpen, 24-Aug-2016. [Online]. Available: https://www.intechopen.com/books/emerging-concepts-in-analysis-andapplications-of-hydrogels/an-introduction-to-hydrogels-and-some-recentapplications. [Accessed: 28-Dec-2019].

[72] M. N. O. Sadiku, Elements of electromagnetics, 5th ed. New York, NY: Oxford University Press, 2010.

[73] X. Qing and Z. N. Chen, "Characteristics of a Metal-Backed Loop Antenna and its Application to a High-Frequency RFID Smart Shelf," in IEEE Antennas and Propagation Magazine, vol. 51, no. 2, pp. 26-38, April 2009.

[74] R. Meys and F. Janssens, "Measuring the impedance of balanced antennas by an Sparameter method", IEEE Antennas and Propagation Magazine, vol. 40, no. 6, pp. 62-65, 1998.

[75] X. Qing, C. K. Goh and Z. N. Chen, "Impedance Characterization of RFID Tag Antennas and Application in Tag Co-Design," in IEEE Transactions on Microwave Theory and Techniques, vol. 57, no. 5, pp. 1268-1274, May 2009.

[76] X. Qing, C. K. Goh and Z. N. Chen, "Measurement of UHF RFID tag antenna impedance," 2009 IEEE International Workshop on Antenna Technology, Santa Monica, CA, 2009, pp. 1-4.

[77] Atecorp.com.

[Online]. Available: https://www.atecorp.com/atecorp/media/pdfs/data-sheets/ah-systems-sas571_datasheet.pdf. [Accessed: 27- Feb- 2019].

[78] X. Yi, T. Wu, Y. Wang and M. Tentzeris, "Sensitivity Modeling of an RFID-Based Strain-Sensing Antenna With Dielectric Constant Change", IEEE Sensors Journal, vol. 15, no. 11, pp. 6147-6155, 2015.

[79] "Materials for printed circuit boards," NANOTECH ELEKTRONIK. [Online]. Available: http://www.nanotech-elektronik.pl/index.php/en/info/materials. [Accessed: 11-Jan-2020]. 
[80] Burt, S., Finney, N. and Young, J. (2018). Fringe Field of Parallel Plate Capacitor. [online] Srjcstaff.santarosa.edu. Available at: http://srjcstaff.santarosa.edu/ yataiiya/UNDER_GRAD_RESEARCH/Fringe\%20F ield\%20of\%20Parallel\%20Plate\%20Capacitor.pdf [Accessed 19 Jun. 2018].

[81] Rrtcadd.org. (2019). Water Amounts in Fruits and Vegetables. [online] Available at: http://rrtcadd.org/resources/Advocacy/Water-Amounts-in-Fruits-and-Vegetables--Handout-Week-10.pdf [Accessed 12 Jan. 2019].

[82] Slideshare.net. (2019). Packing of harvested fruits and vegetables. [online] Available at: https://www.slideshare.net/GayaniRasangika/packing-of-harvestedfruits-and-vegetables [Accessed 12 Jan. 2019].

[83] Saghlatoon, H., Sydanheimo, L., Ukkonen, L. and Tentzeris, M. (2014). Optimization of Inkjet Printing of Patch Antennas on Low-Cost Fibrous Substrates. IEEE Antennas and Wireless Propagation Letters, 13, pp.915-918.

[84] Blattenberger, K. (2019). Dielectric Constant, Strength, \& Loss Tangent - RF Cafe. [online] Rfcafe.com. Available at: http://www.rfcafe.com/references/electrical/dielectric-constants-strengths.htm [Accessed 12 Jan. 2019].

[85] A. Naini and M. Green, "Fringing fields in a parallel-plate capacitor," American Journal of Physics, vol. 45, no. 9, pp. 877-879, Apr. 1977.

[86] M. N. O. Sadiku, Elements of electromagnetics, 5th ed. New York, NY: Oxford University Press, 2010.

[87] Y. Shafiq, J. Henricks, C. P. Ambulo, T. H. Ware and S. V. Georgakopoulos, "A Passive RFID Temperature Sensing Antenna With Liquid Crystal Elastomer Switching," in IEEE Access, vol. 8, pp. 24443-24456, 2020.

[88] Fan Yang and Y. Rahmat-Samii, "Patch antennas with switchable slots (PASS) in wireless communications: concepts, designs, and applications," in IEEE Antennas and Propagation Magazine, vol. 47, no. 2, pp. 13-29, April 2005.

[89] Volakis, J. (2019). ANTENNA ENGINEERING HANDBOOK. 5th ed. New York: McGraw-Hill Education, pp.829-837.

[90] Mouser.com. (2019). ADGM1004 MEMS Switch with Integrated Driver. [online] Available at: https://www.mouser.com/datasheet/2/609/adgm1004-1503081.pdf [Accessed 4 Aug. 2019].

[91] Minicircuits.com. (2019). High Isolation Switch. [online] Available at: https://www.minicircuits.com/pdfs/KSWHA-1-20+.pdf [Accessed 4 Aug. 2019]. 
[92] "D2F Ultra Subminiature Basic Switch", Media.digikey.com, 2019. [Online]. Available:

https://media.digikey.com/pdf/Data\%20Sheets/Omron\%20PDFs/D2F_0318_DS.p df. [Accessed: 07- Feb- 2019].

[93] “TMM® Thermoset Microwave Materials," www.rogerscorp.com. [Online]. Available: https://rogerscorp.com/-/media/project/rogerscorp/documents/advancedconnectivity-solutions/english/data-sheets/tmm-thermoset-laminate-data-sheettmm3----tmm4----tmm6----tmm10----tmm10i----tmm13i.pdf. [Accessed: 25-Dec2019].

[94] A. Thakur, M. Chauhan, and M. Kumar, "Effect of Substrate relative dielectric constant on Bandwidth characteristics of Line feed Rectangular Patch Antenna," International Journal of Engineering Science Invention Research \& Development, vol. 1, no. X, Apr. 2015.

[95] R. Garg, P. Bhartia, I. J. Bahl, and A. Ittipiboon, Microstrip antenna design handbook. Boston: Artech House, 2001.

[96] D. Orban and G. Moernaut, "The Basics of Patch Antennas, Updated", Orbanmicrowave.com, 2005. [Online]. Available: http://orbanmicrowave.com/wp-content/uploads/2014/12/Orban-Patch-Antennas2009-rev.pdf. [Accessed: 19- Feb- 2019].

[97] "The benefits of circuit and antenna co-simulation for optimising wideband structures," IEE Wideband and Multi-band Antennas and Arrays 2005 (Ref. No. 2005/11059), Birmingham, UK, 2005, pp. 191-195.

[98] Syuhaimi, Kassim \& AbdulMalek, MohamedFareq. (2010). EM/Circuit CoSimulation: A Highly Accurate Method for Microwave Amplifier Design. Universal Journal of Computer Science and Engineering Technology.

[99] K. Rawat and F. Ghannouchi, "Dual-band matching technique based on dualcharacteristic impedance transformers for dual-band power amplifiers design," IET Microwaves, Antennas \& Propagation, vol. 5, no. 14, p. 1720, 2011.

[100] X. Zheng, Y. Liu, S. Li, C. Yu, Z. Wang and J. Li, "A DUAL-BAND IMPEDANCE TRANSFORMER USING PI-SECTION STRUCTURE FOR FREQUENCYDEPENDENT COMPLEX LOADS", Progress In Electromagnetics Research C, vol. 32, pp. 11-26, 2012. Available: 10.2528/pierc12052909.

[101] Ming-Lin Chuang, "Dual-Band Impedance Transformer Using Two-Section Shunt Stubs", IEEE Transactions on Microwave Theory and Techniques, vol. 58, no. 5, pp. 1257-1263, 2010. Available: 10.1109/tmtt.2010.2045560. 
[102] M. A. Maktoomi, V. Panwar, M. S. Hashmi and F. M. Ghannouchi, "A dual-band matching network for frequency-dependent complex loads suitable for dual-band RF amplifiers," 2014 IEEE International Microwave and RF Conference (IMaRC), Bangalore, 2014, doi: 10.1109/IMaRC.2014.7038977.

pp.

88-91.

[103] M. A. Nikravan and Z. Atlasbaf, "T-section dual-band impedance transformer for frequency-dependent complex impedance loads," in Electronics Letters, vol. 47, no. 9, $\quad$ pp. 551-553, 28 April 2011. doi: 10.1049/el.2010.7452.

[104] Vishay.com. (2019). High Frequency (up to $40 \mathrm{GHz}$ ) Resistor, Thin Film Surface Mount Chip. [online] Available at: http://www.vishay.com/docs/60093/fcseries.pdf [Accessed 28 Sep. 2019].

[105] S. K. Behera, "Novel Tuned Rectangular Patch Antenna As a Load for Phase Power Combining," Ph.D. dissertation, Dept. Elect. and Telecom. Eng., Jadavpur University, Kolkata, India, 2002. p. 18.

[106] Vaisala.com. (2020). Preventing Condensation. [online] Available at: https://www.vaisala.com/sites/default/files/documents/PreventingCondensation.pdf [Accessed 20 Jan. 2020].

[107] Y. Qian and T. Itoh, "A broadband uniplanar microstrip-to-CPS transition," Proceedings of 1997 Asia-Pacific Microwave Conference.

[108] N. Kaneda, Y. Qian and T. Itoh, "A broad-band microstrip-to-waveguide transition using quasi-Yagi antenna," in IEEE Transactions on Microwave Theory and Techniques, vol. 47, no. 12, pp. 2562-2567, Dec. 1999.

[109] Yuan, L. and Tang, W. (2018). NOVEL MINIATURIZED BROADBAND QUASIYAGI ANTENNAS BASED ON MODIFIED BOWTIES DRIVER FOR WIRELESS TECHNOLOGY APPLICATIONS. Progress In Electromagnetics Research M, 63, pp.151-161.

[110] Eldek, A., Elsherbeni, A. and Smith, C. (2004). Wideband microstrip-fed printed bow-tie antenna for phased-array systems. Microwave and Optical Technology Letters, 43(2), pp.123-126. 
VITA

\section{YOUSUF SHAFIQ}

2005-2009

B.S., Electrical Engineering

Florida International University

Miami, FL

2009-2010

M.S., Electrical Engineering

Florida International University

Miami, FL

2018-2020

Ph.D. Candidate, Electrical Engineering

Florida International University

Miami, Florida

\section{PUBLICATIONS and PRESENTATIONS}

[1] Y. Shafiq, J. Henricks, C. P. Ambulo, T. H. Ware, and S. V. Georgakopoulos, “A Battery-Free Temperature Sensor with Liquid Crystal Elastomer Switching Between RFID Chips," in IEEE Access [In Review: Submitted Mar. 2020]

[2] Y. Shafiq, J. Henricks, C. P. Ambulo, T. H. Ware and S. V. Georgakopoulos, "A Passive RFID Temperature Sensing Antenna With Liquid Crystal Elastomer Switching," in IEEE Access, vol. 8, pp. 24443-24456, 2020.

[3] Y. Shafiq, J. S. Gibson, H. Kim, C. P. Ambulo, T. H. Ware and S. V. Georgakopoulos, "A Reusable Battery-Free RFID Temperature Sensor," in IEEE Transactions on Antennas and Propagation, vol. 67, no. 10, pp. 6612-6626, Oct. 2019.

[4] S. V. Georgakopoulos and Y. Shafiq, "Passive RFID Real-Time Sensors Based On Programmable Liquid Crystal Elastomers," Ref. No.: FIU.329P. (provisional patent filed on Jul. 12, 2019)

[5] Y. Shafiq, S. V. Georgakopoulos, H. Kim, C. P. Ambulo and T. H. Ware, "A Patch Antenna with Liquid Crystal Elastomer Switching for Passive RFID Temperature Sensing," 2019 IEEE International Symposium on Antennas and Propagation and USNC-URSI Radio Science Meeting, Atlanta, GA, USA, 2019, pp. 1891-1892.

[6] Y. Shafiq, J. Gibson, S. V. Georgakopoulos, H. Kim, C. P. Ambulo and T. H. Ware, "A Novel Passive RFID Temperature Sensor," 2018 IEEE International Symposium on Antennas and Propagation \& USNC/URSI National Radio Science Meeting, Boston, MA, 2018, pp. 1863-1864.

[7] Y. Shafiq, J. Gibson, S. V Georgakopoulos, H. Kim, C. P. Ambulo and T. H. Ware, "A Novel Passive RFID Temperature Sensor Using Liquid Crystal Elastomers," 2018 IEEE International Symposium on Antennas and Propagation \& USNC/URSI National Radio Science Meeting, Boston, MA, 2018, pp. 2013-2014. 